\title{
SUSTAINABLE CONSUMERISM
}

NE MARKET

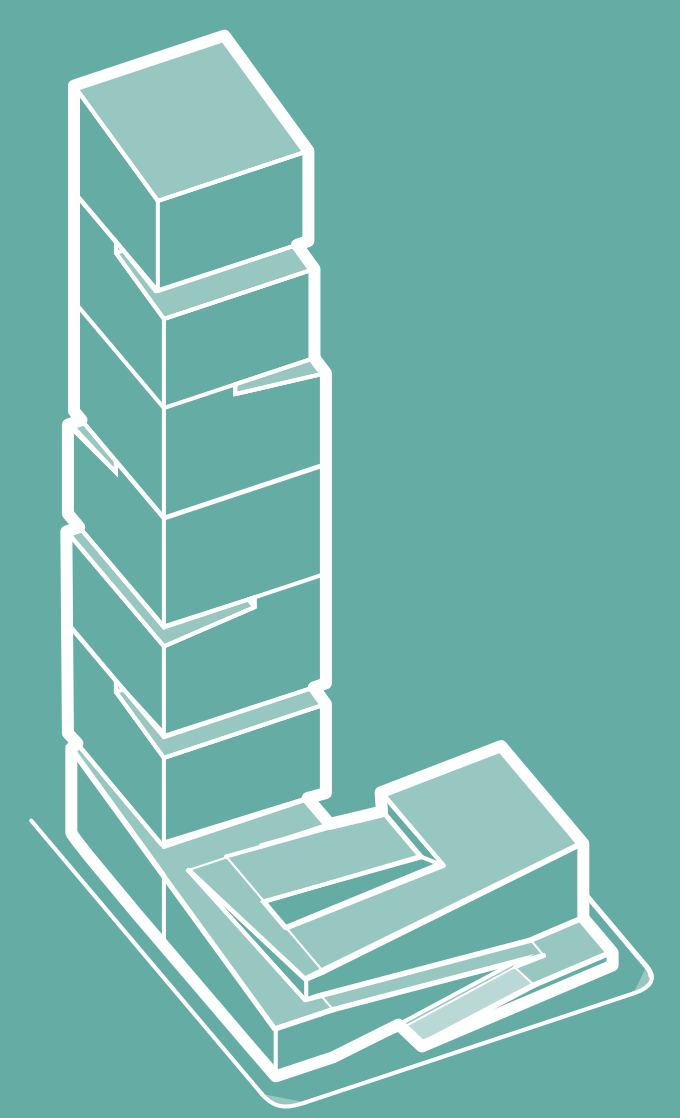

4784170 Sibing Emily Yang Complex Project Graduation Studio Group 2 


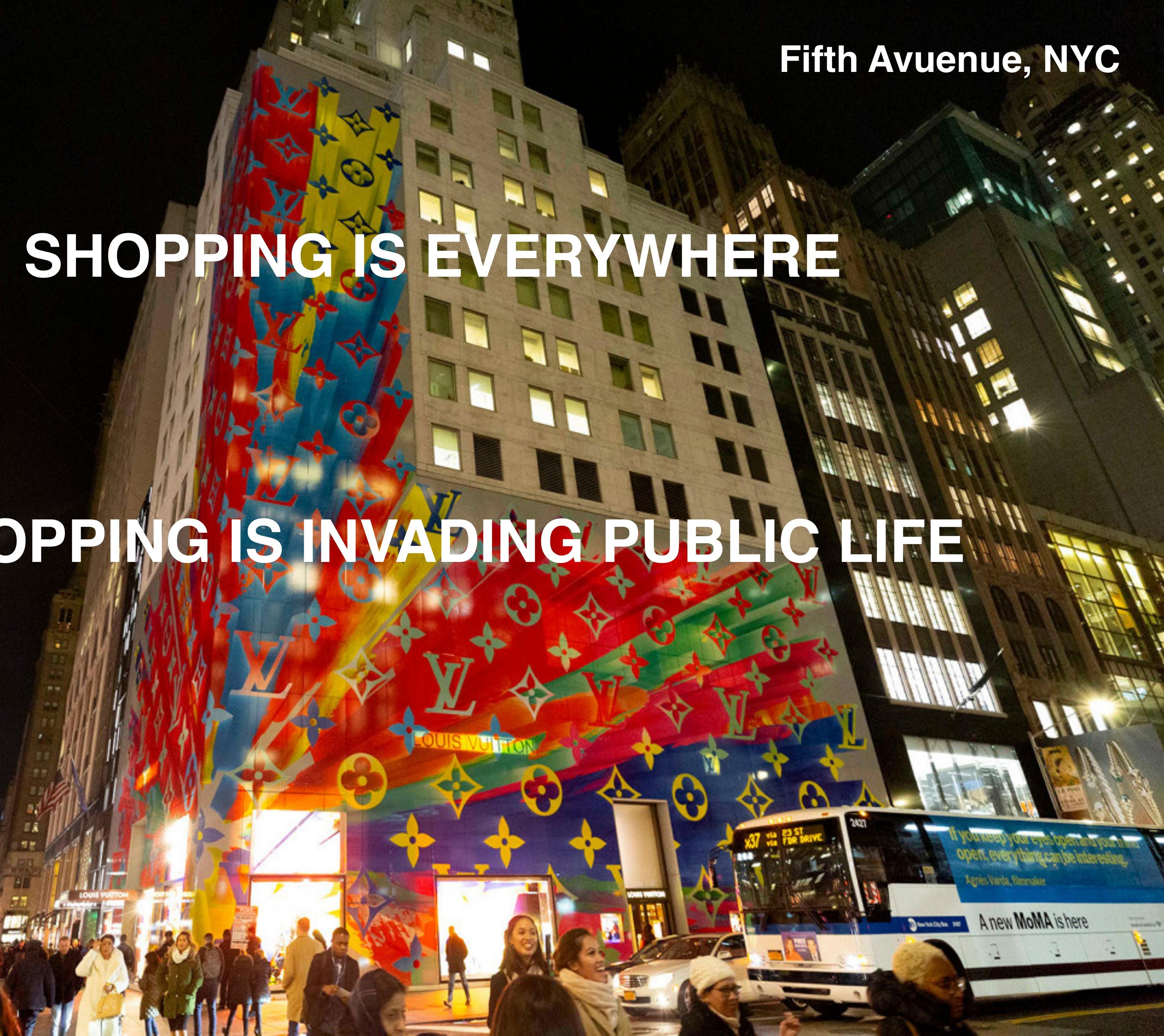




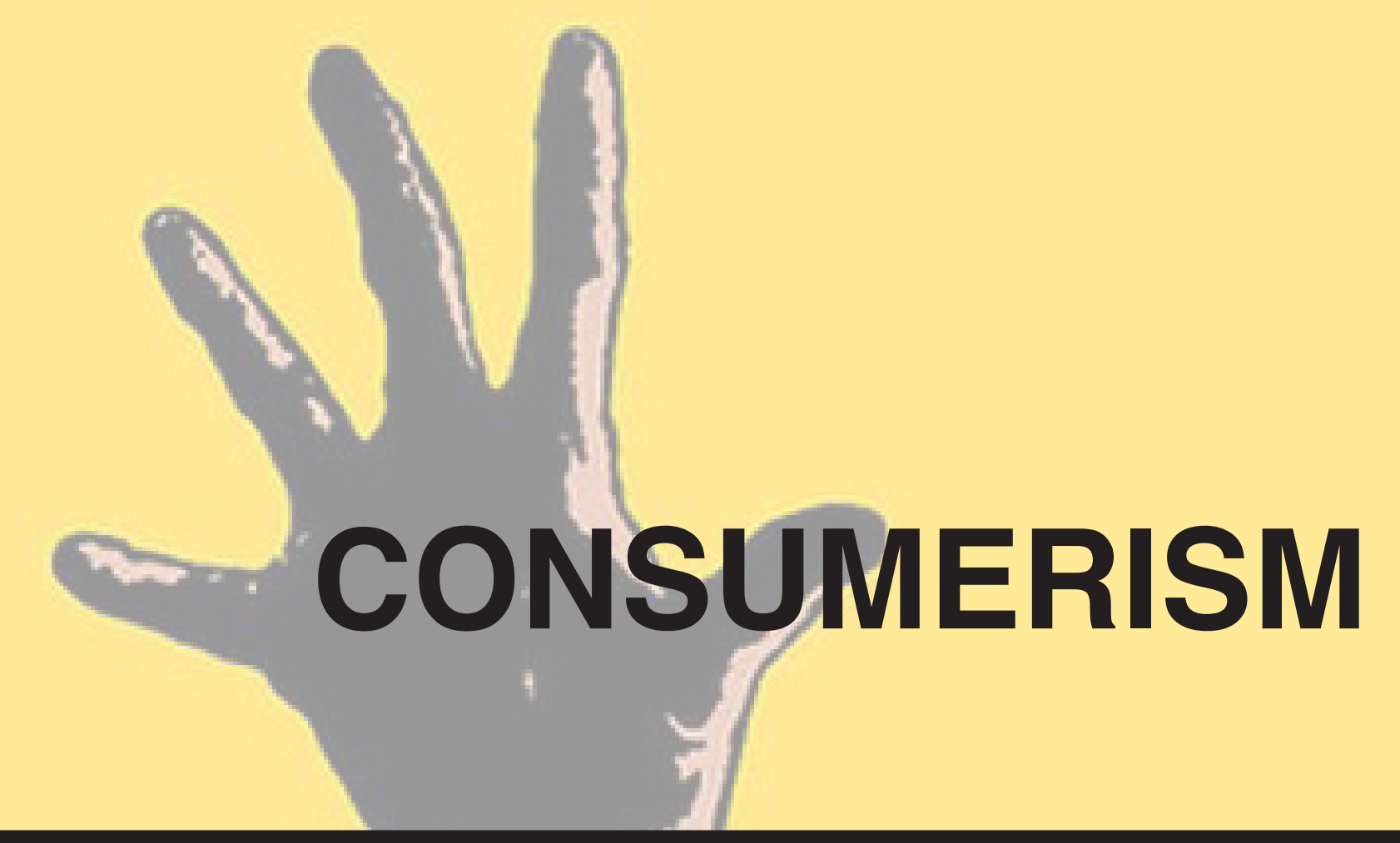

is encouracing the accuisition of goods and services in

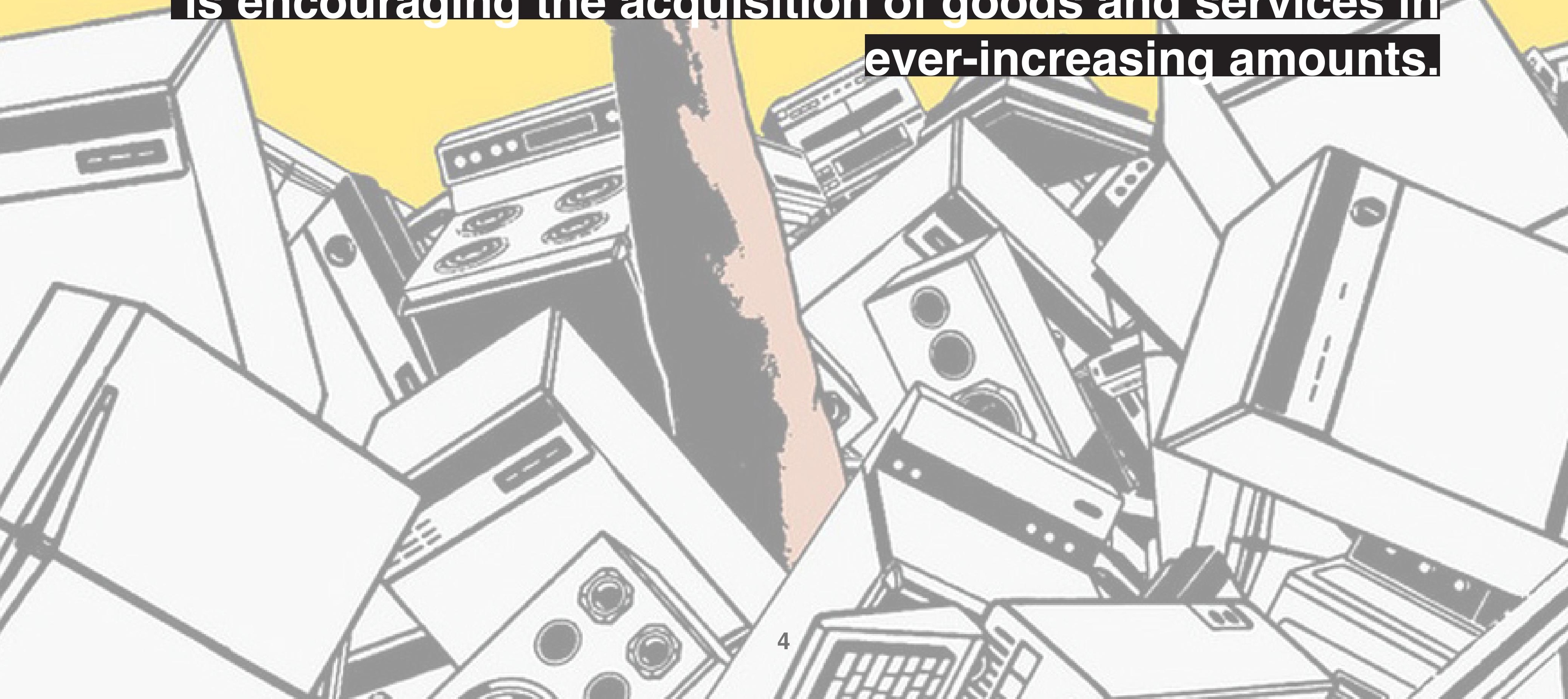




\section{RECREATIONAL SHOPPING}

\section{NECESSARY SHOPPING}
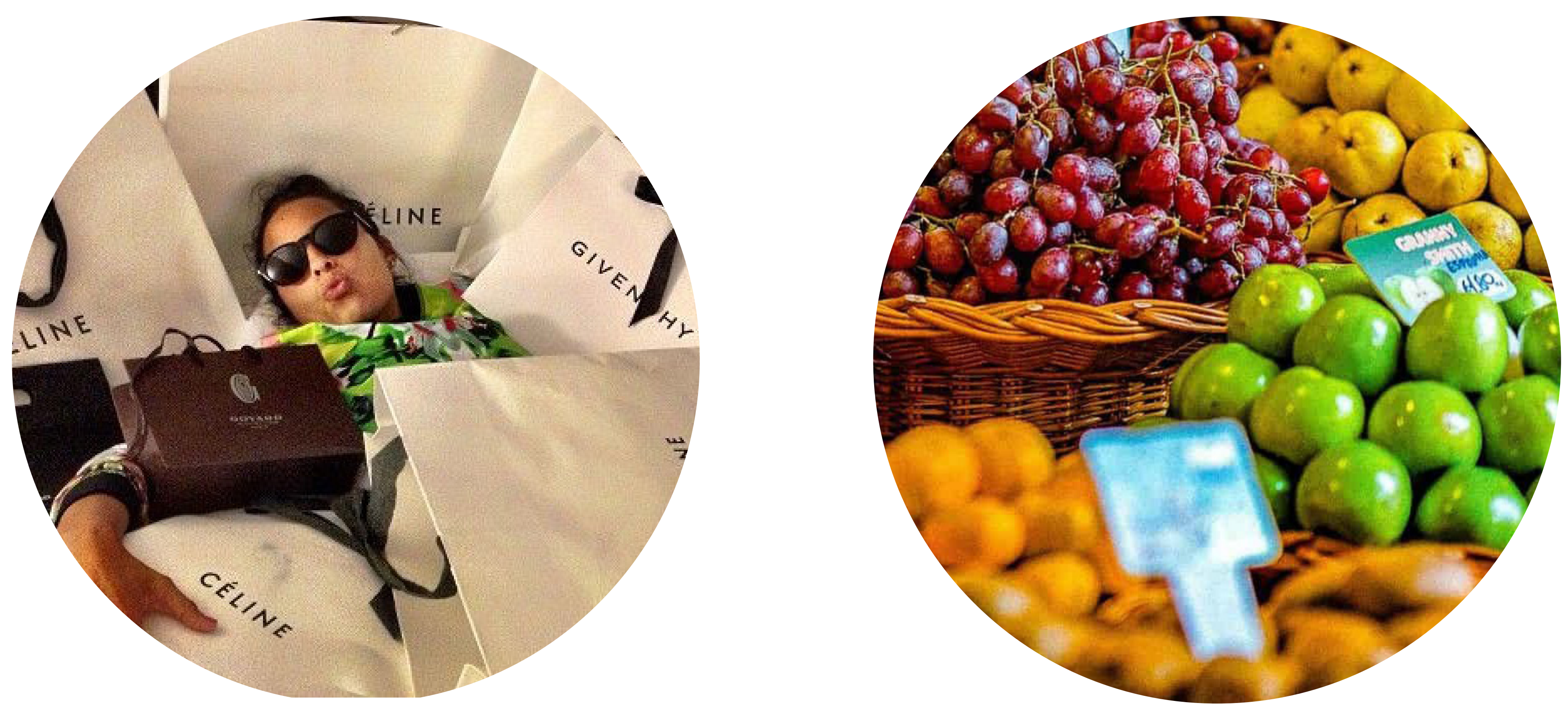


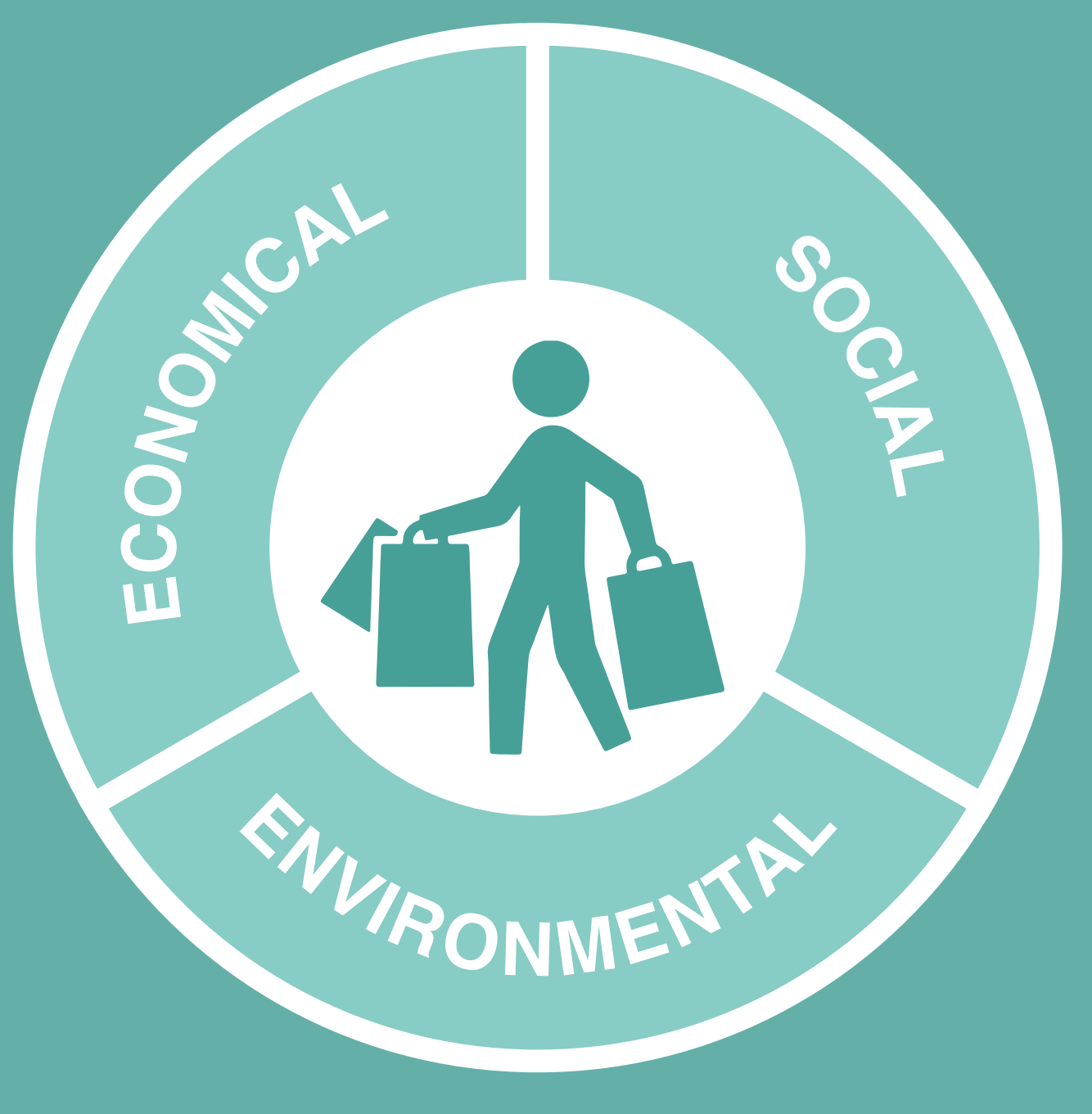



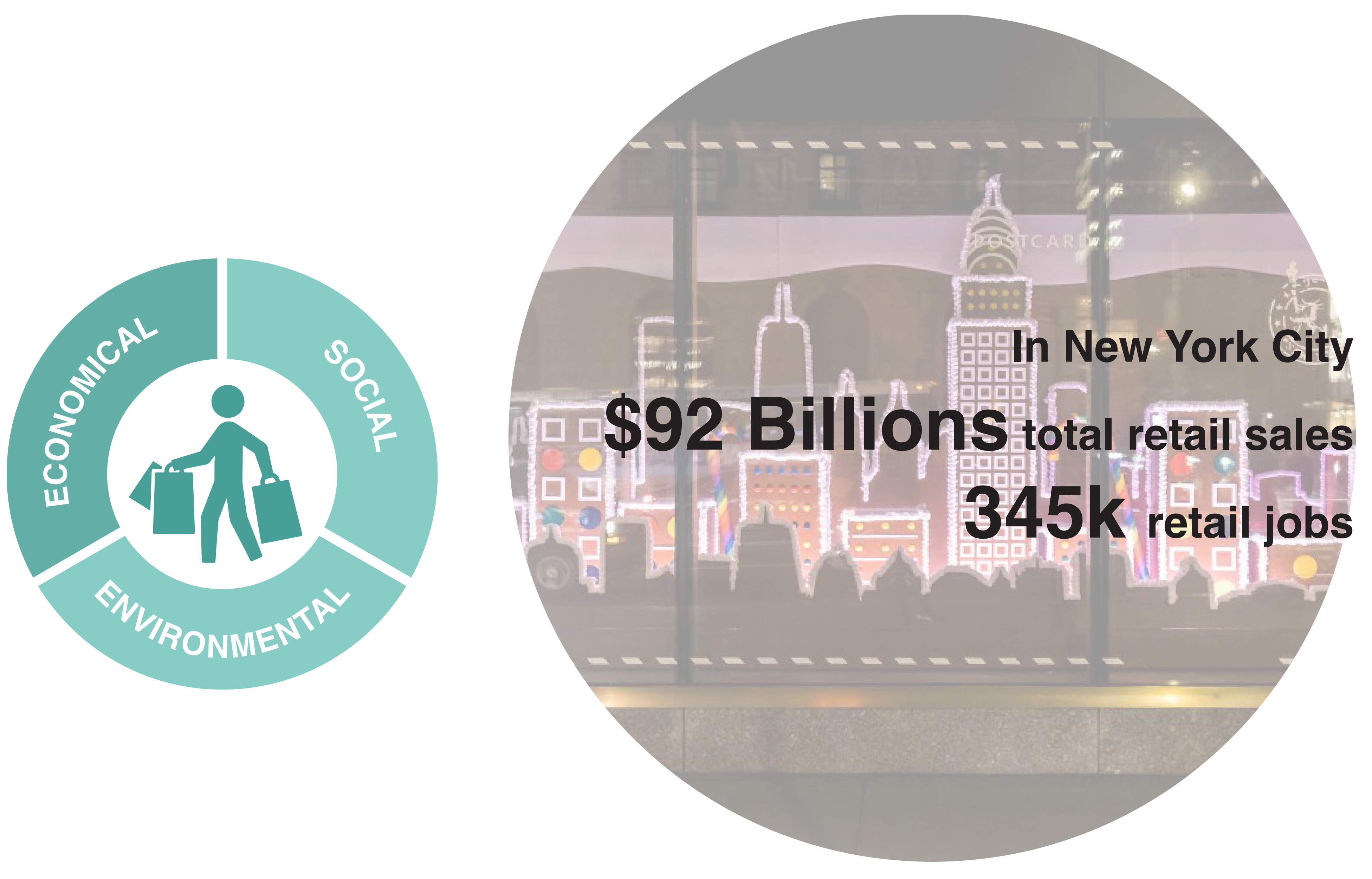

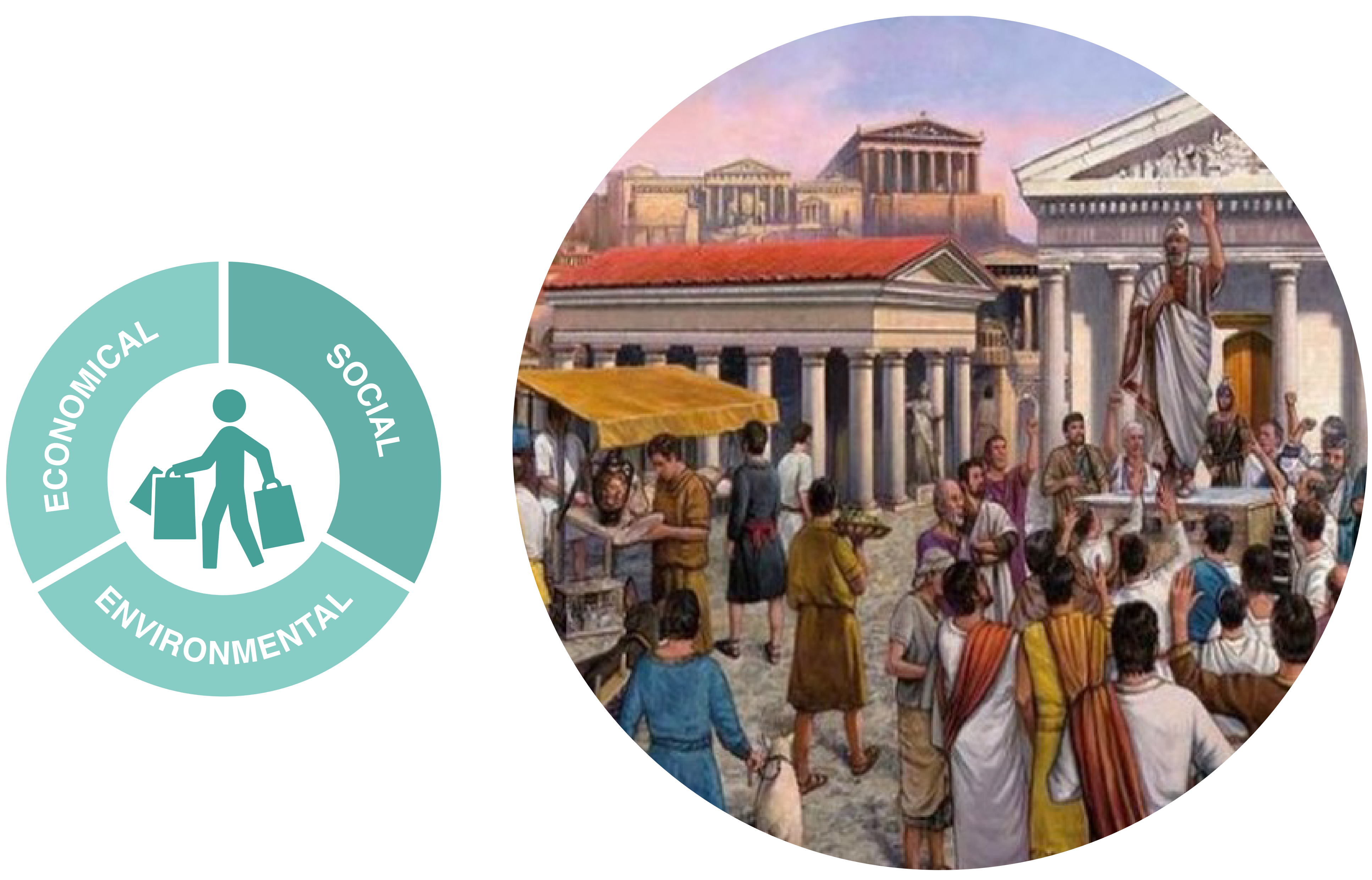

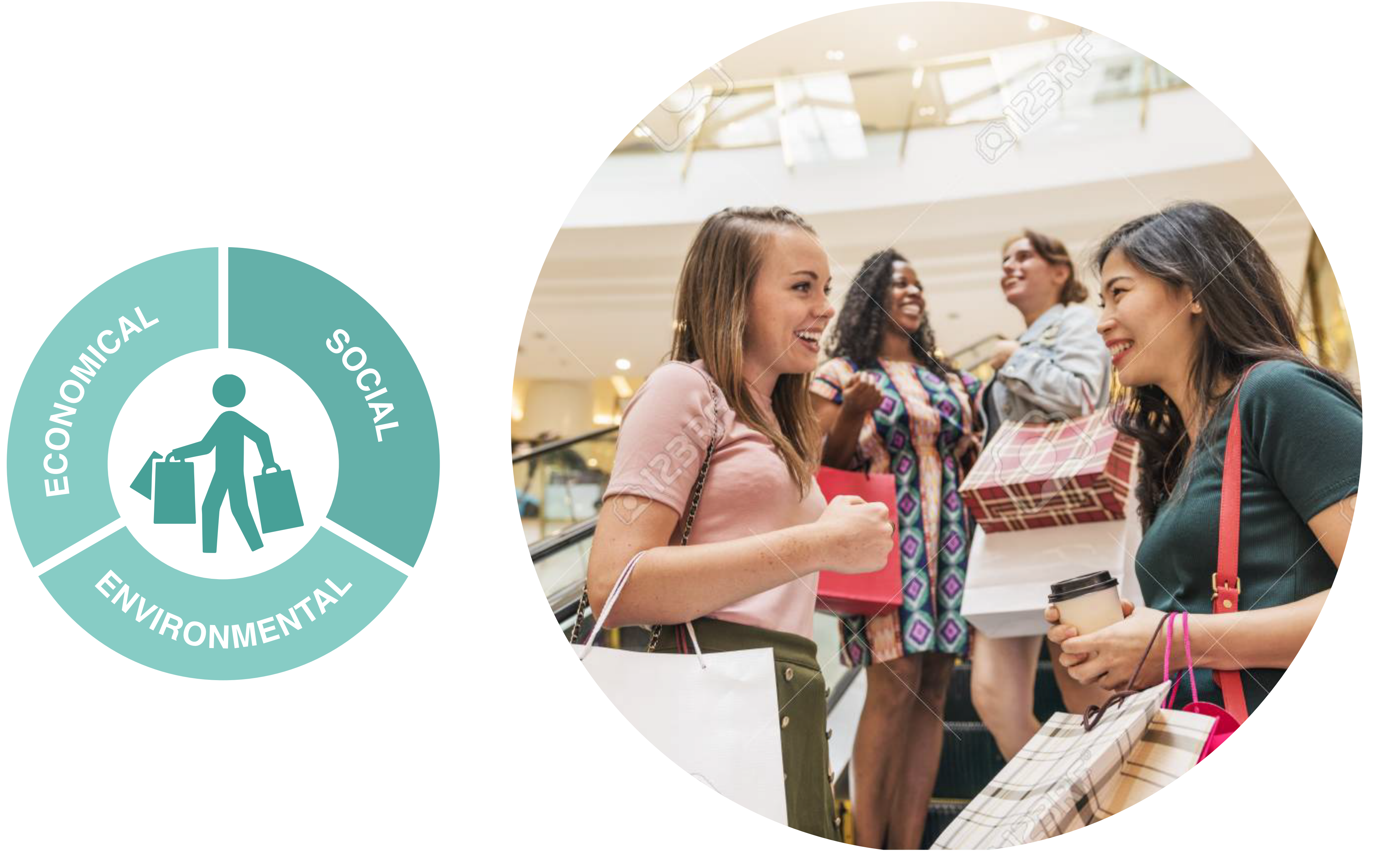

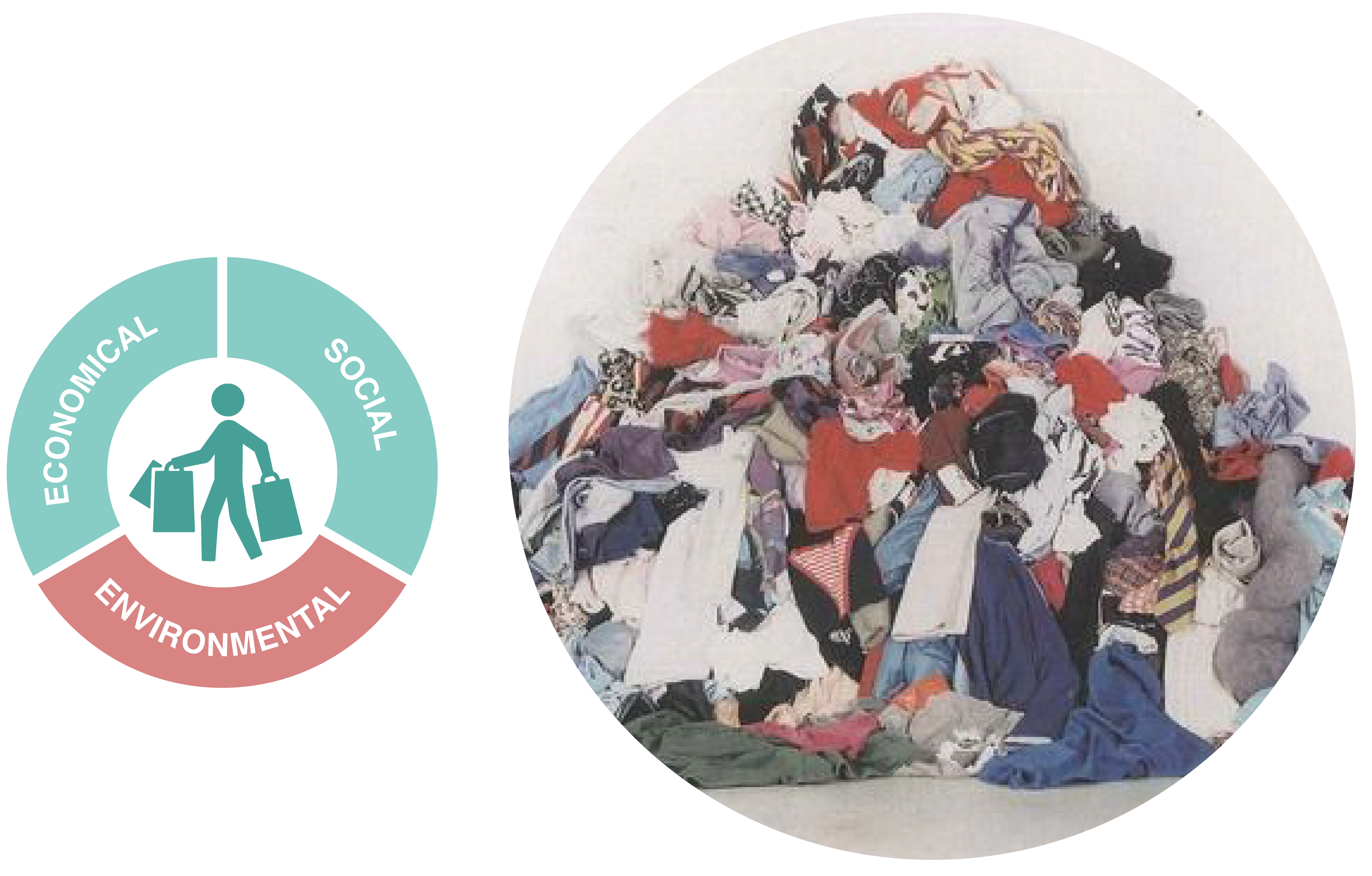
It takes over 30,000 litres to create $1 \mathrm{~kg}$ of cotton. 1 cotton shirt uses approximately 2,700 litres of water. Up to 600 litres of water can be re-

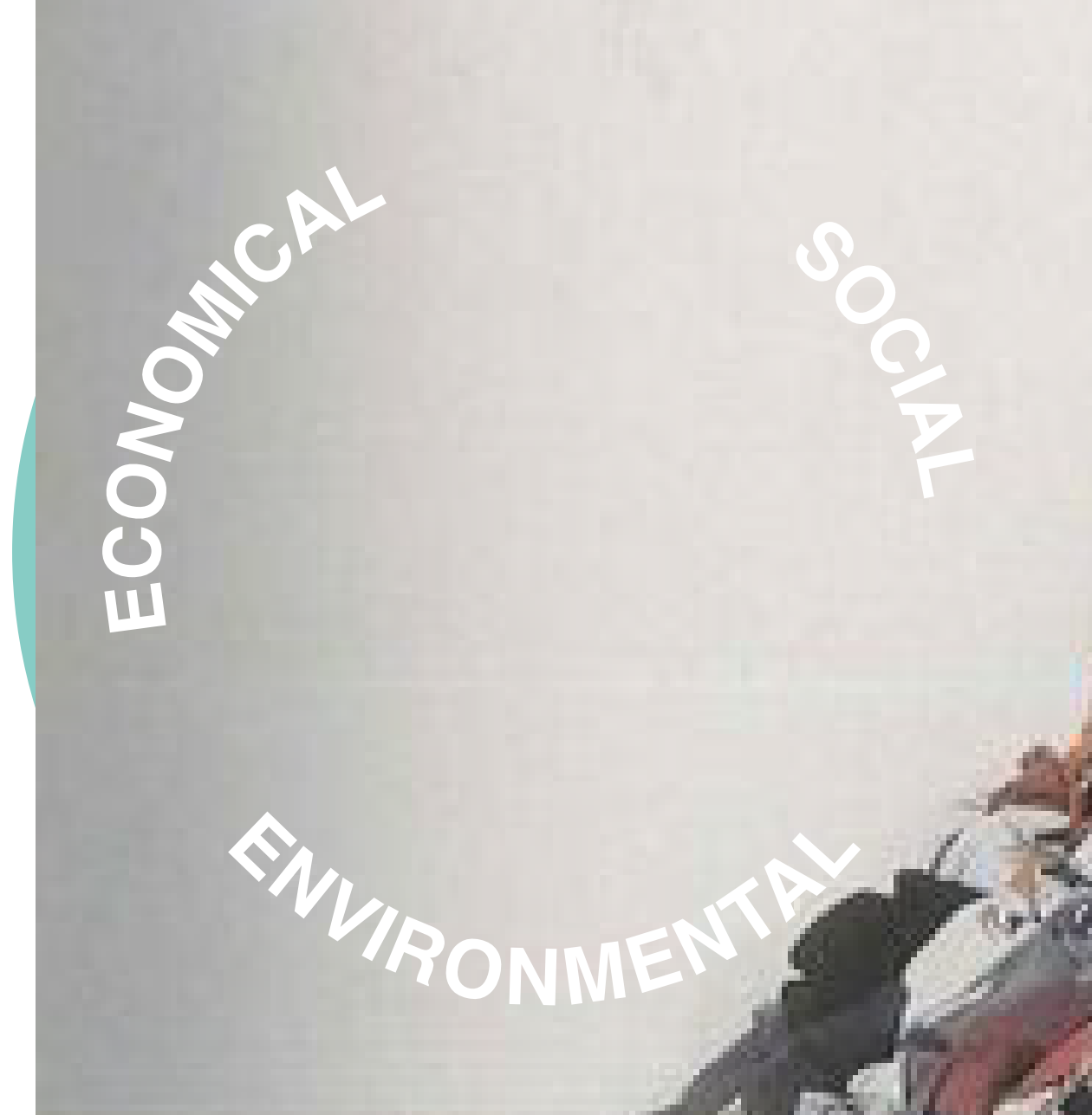

SHOPPING $=$ CONSUMING 

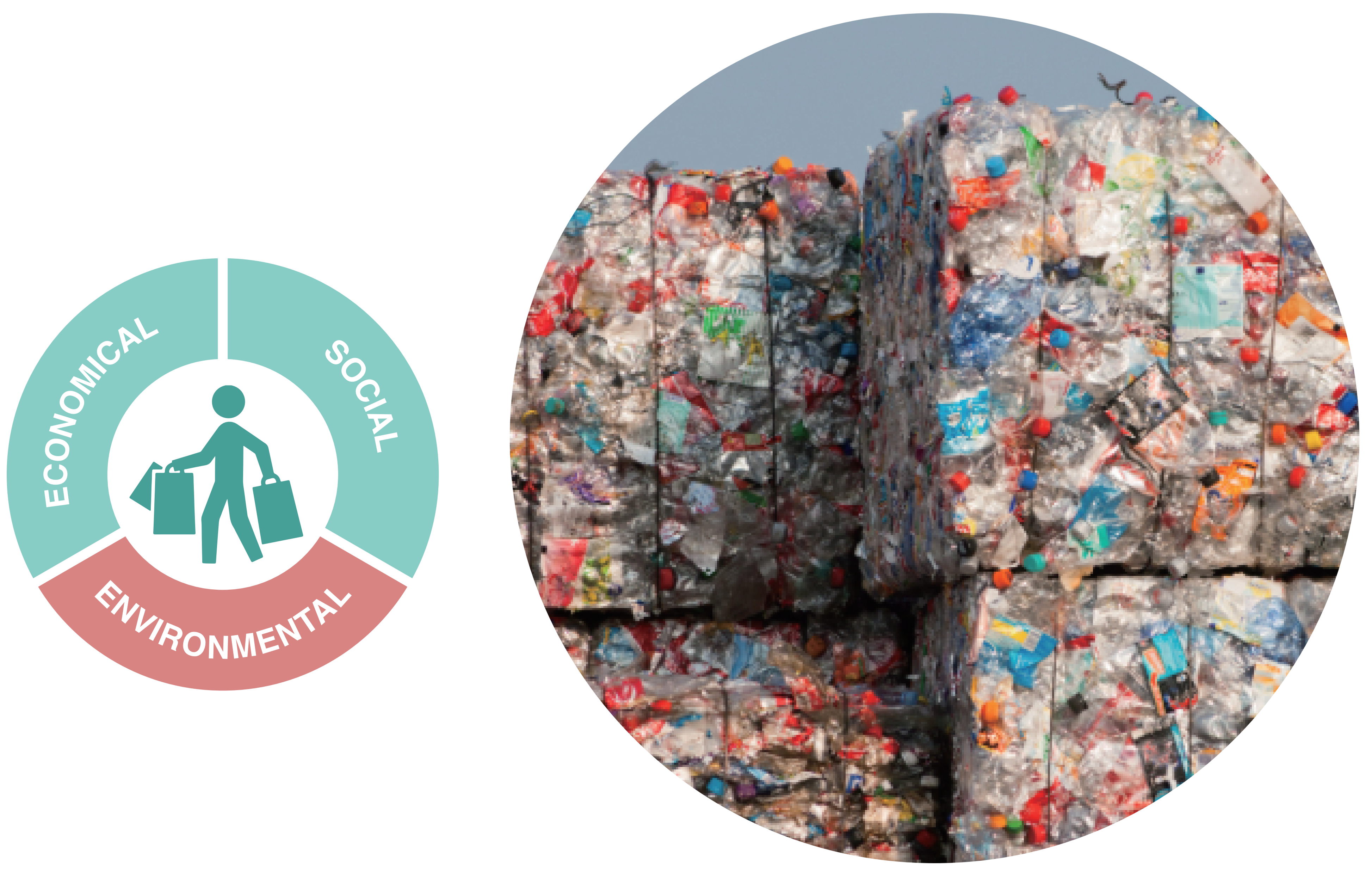

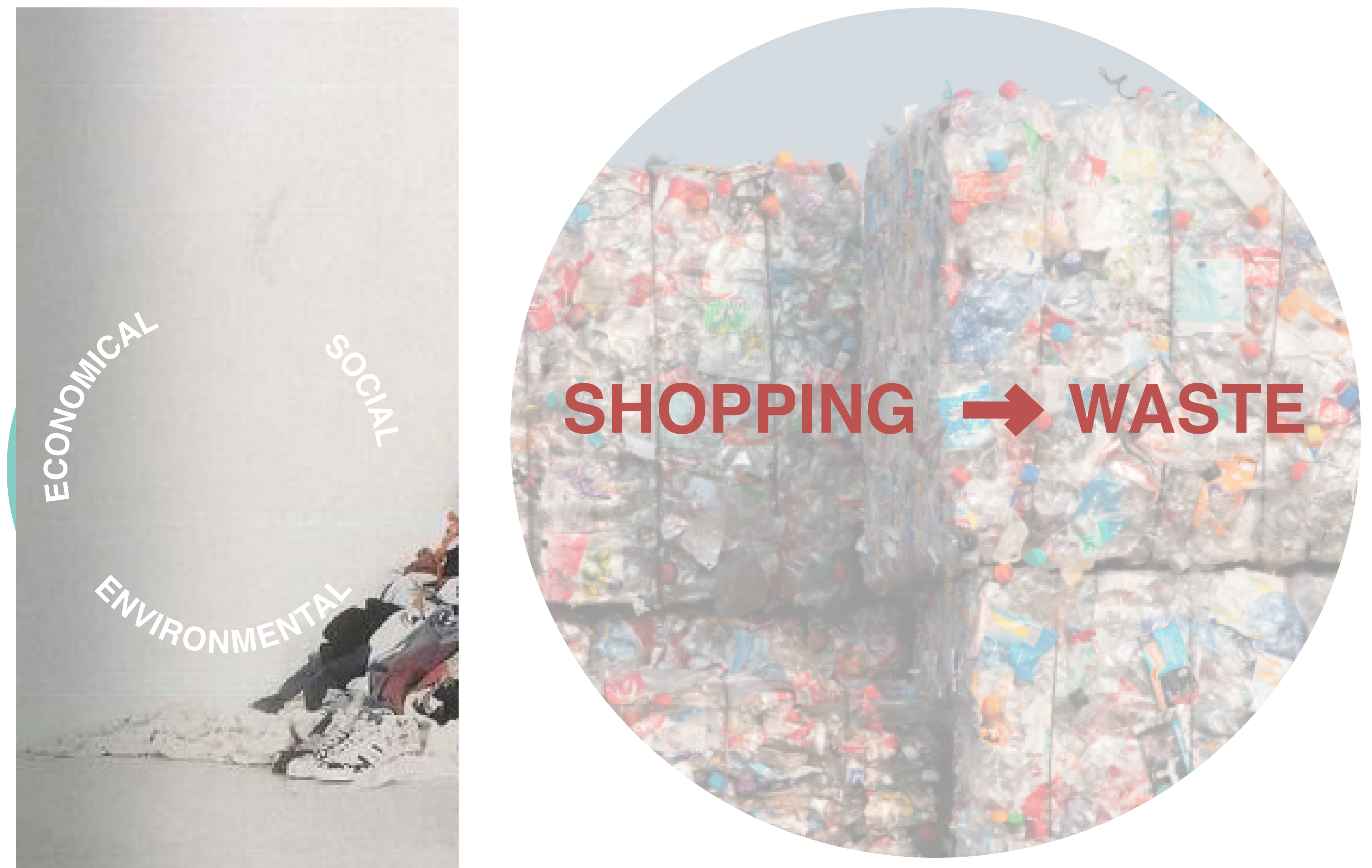


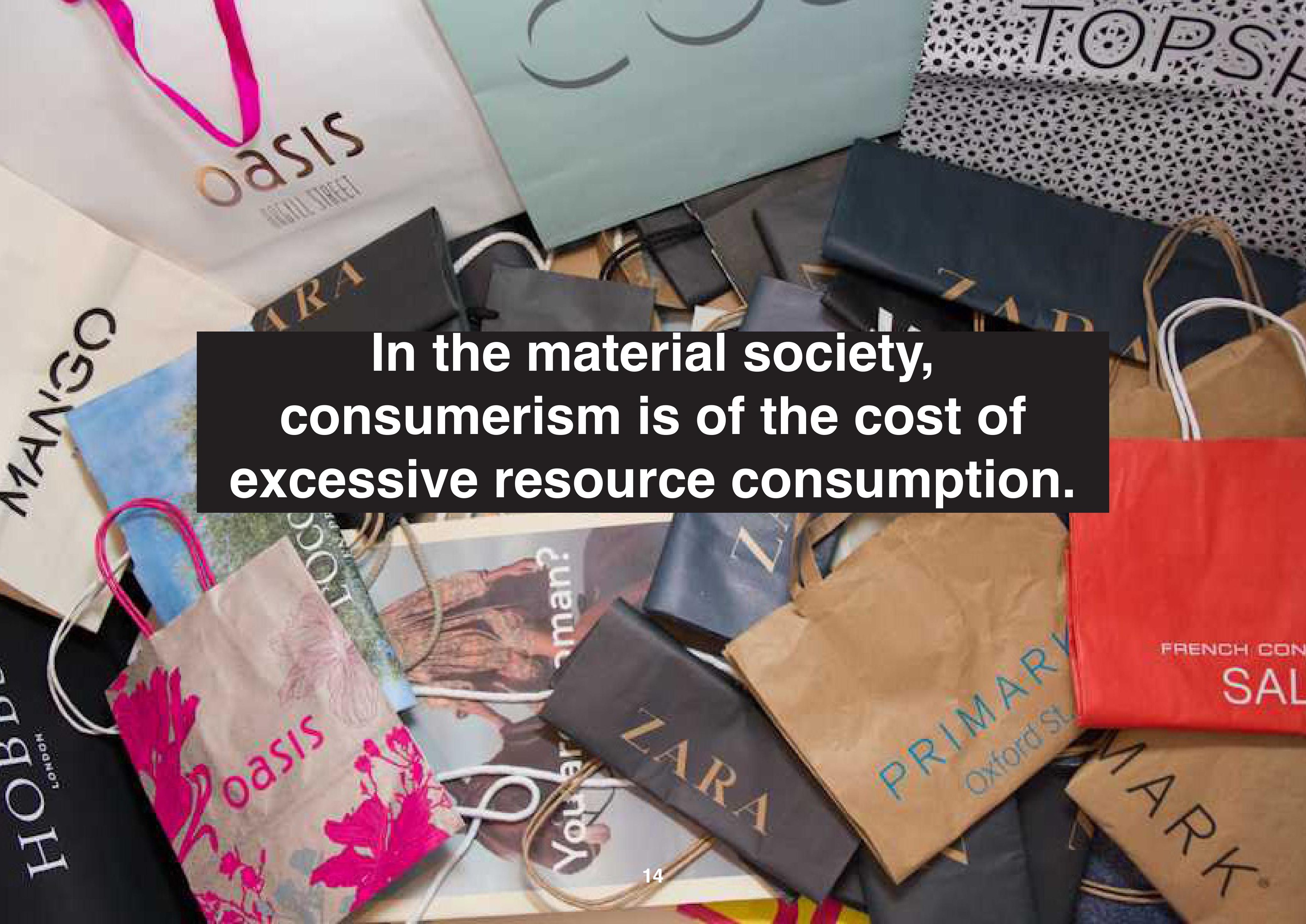




\section{RESEARCH QUESTION}

\section{How to make consumerism socially, economically and environmentally sustainable?}




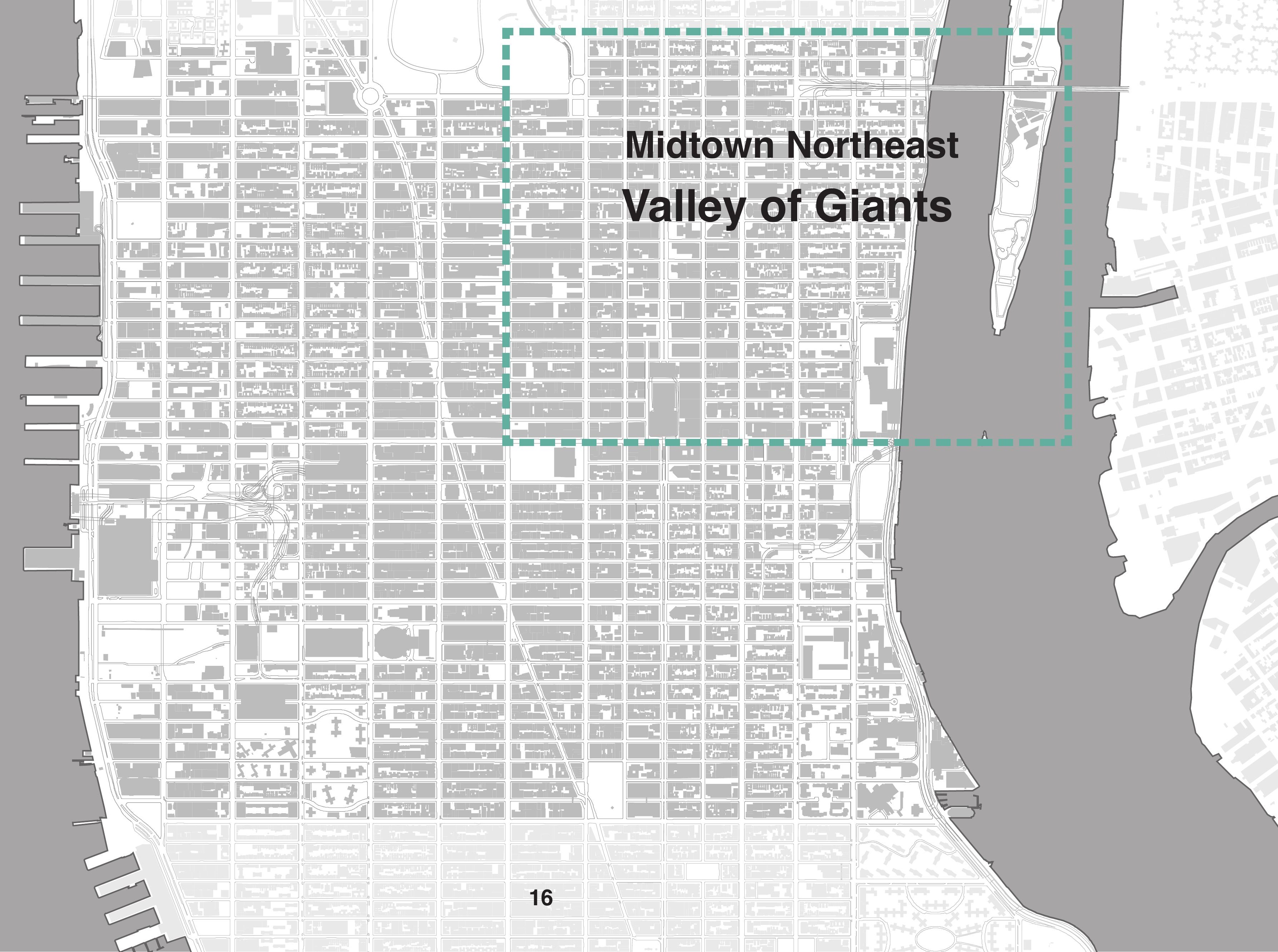




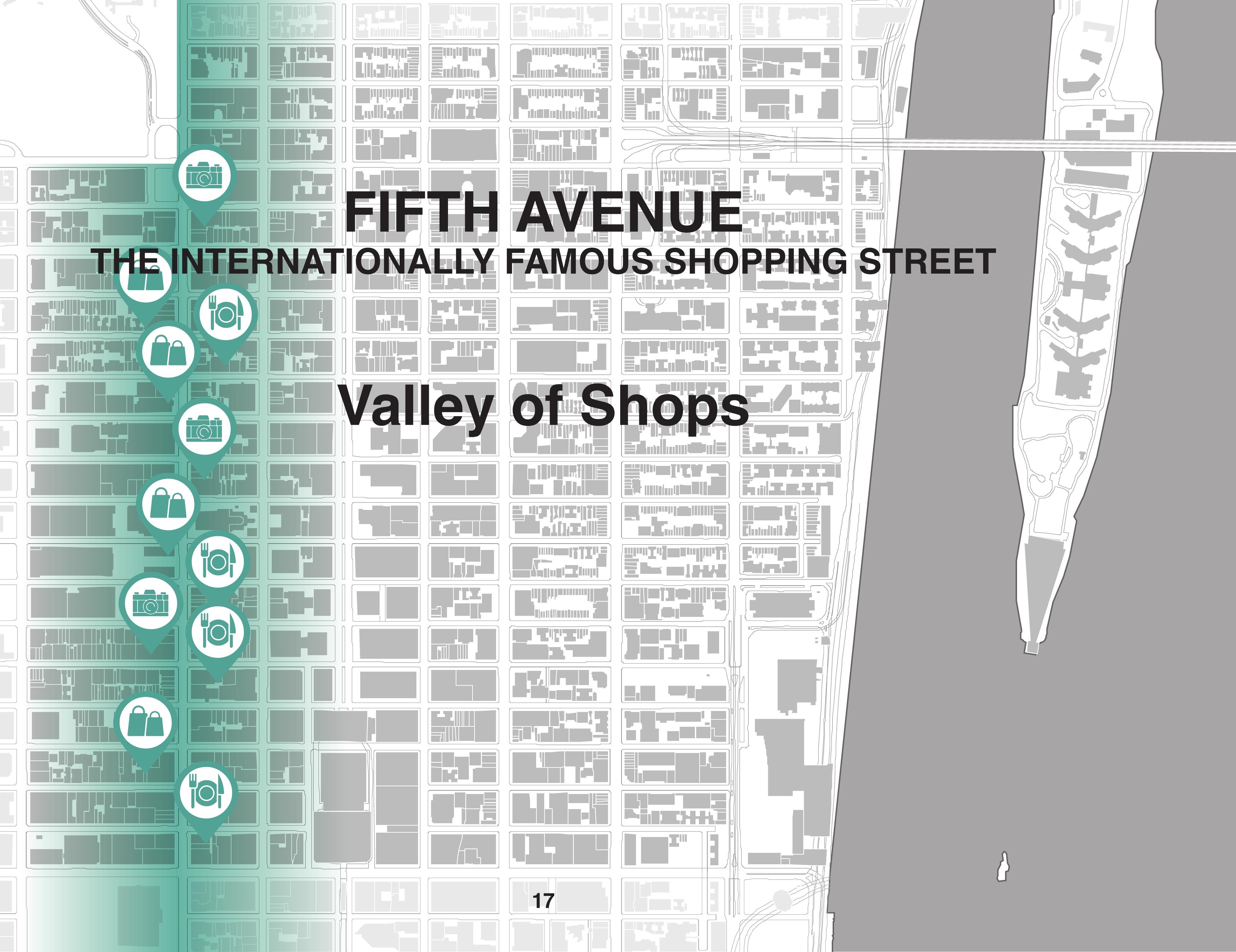




\section{LUXURY BRANDS}

LOUIS VUITTON

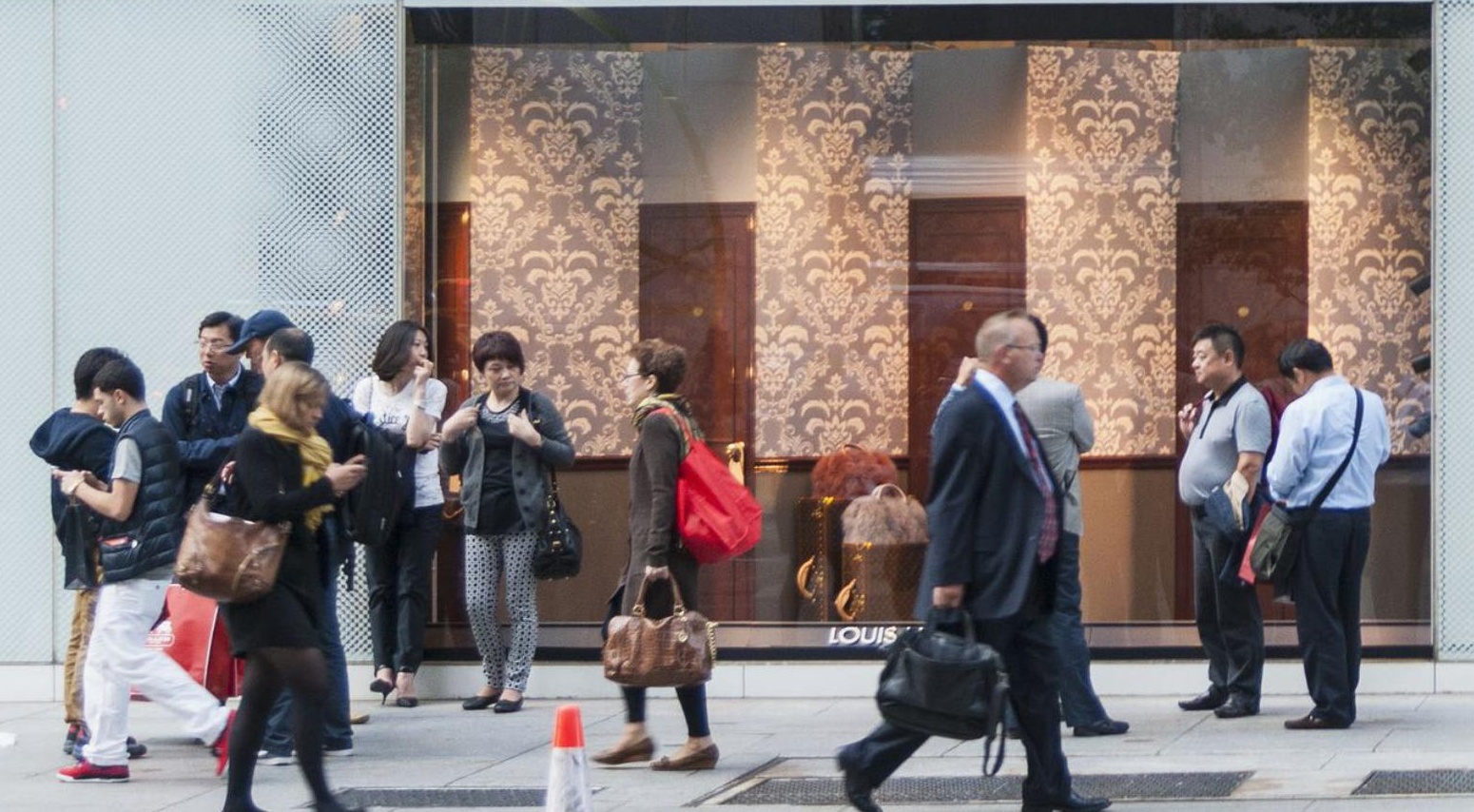




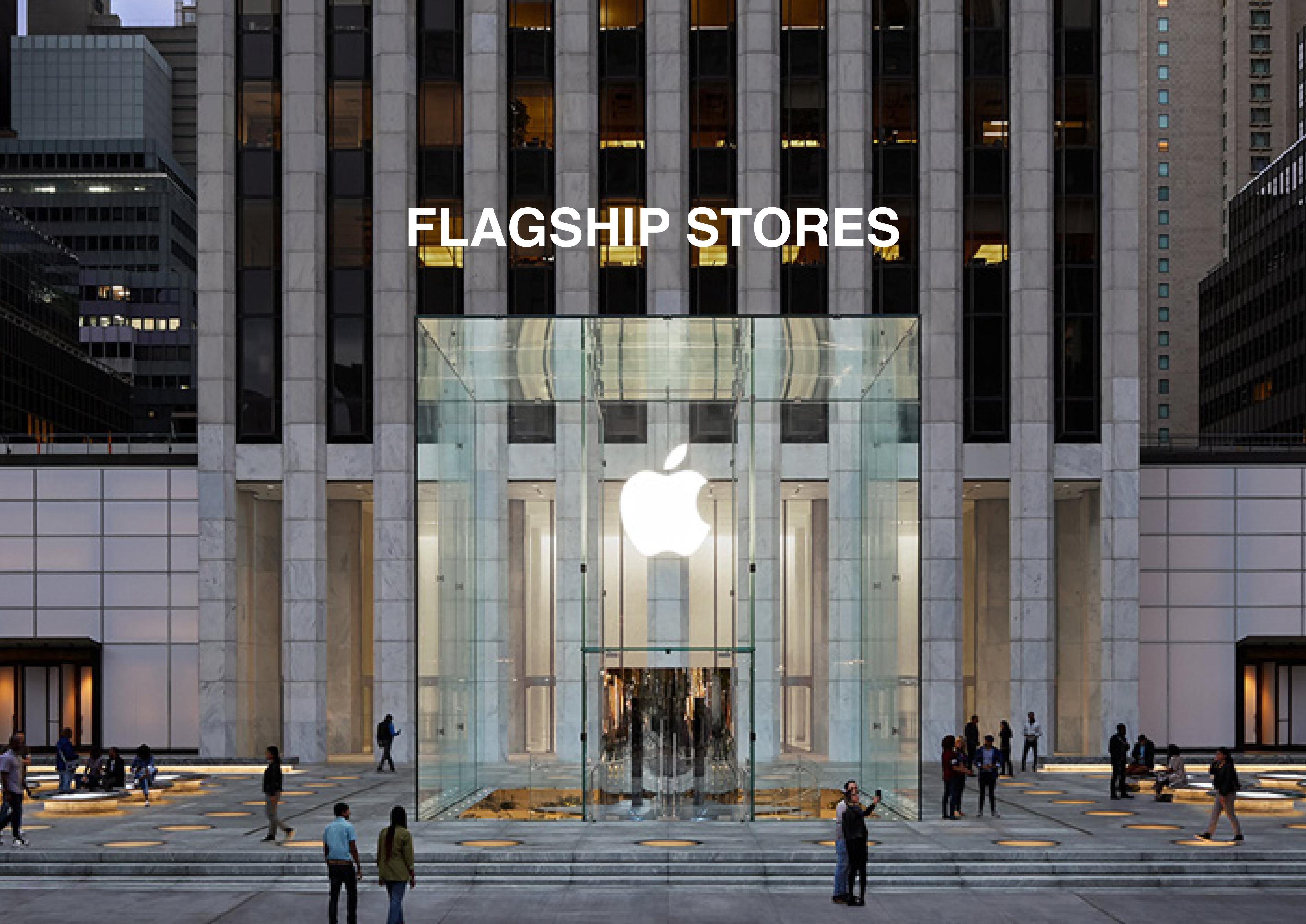




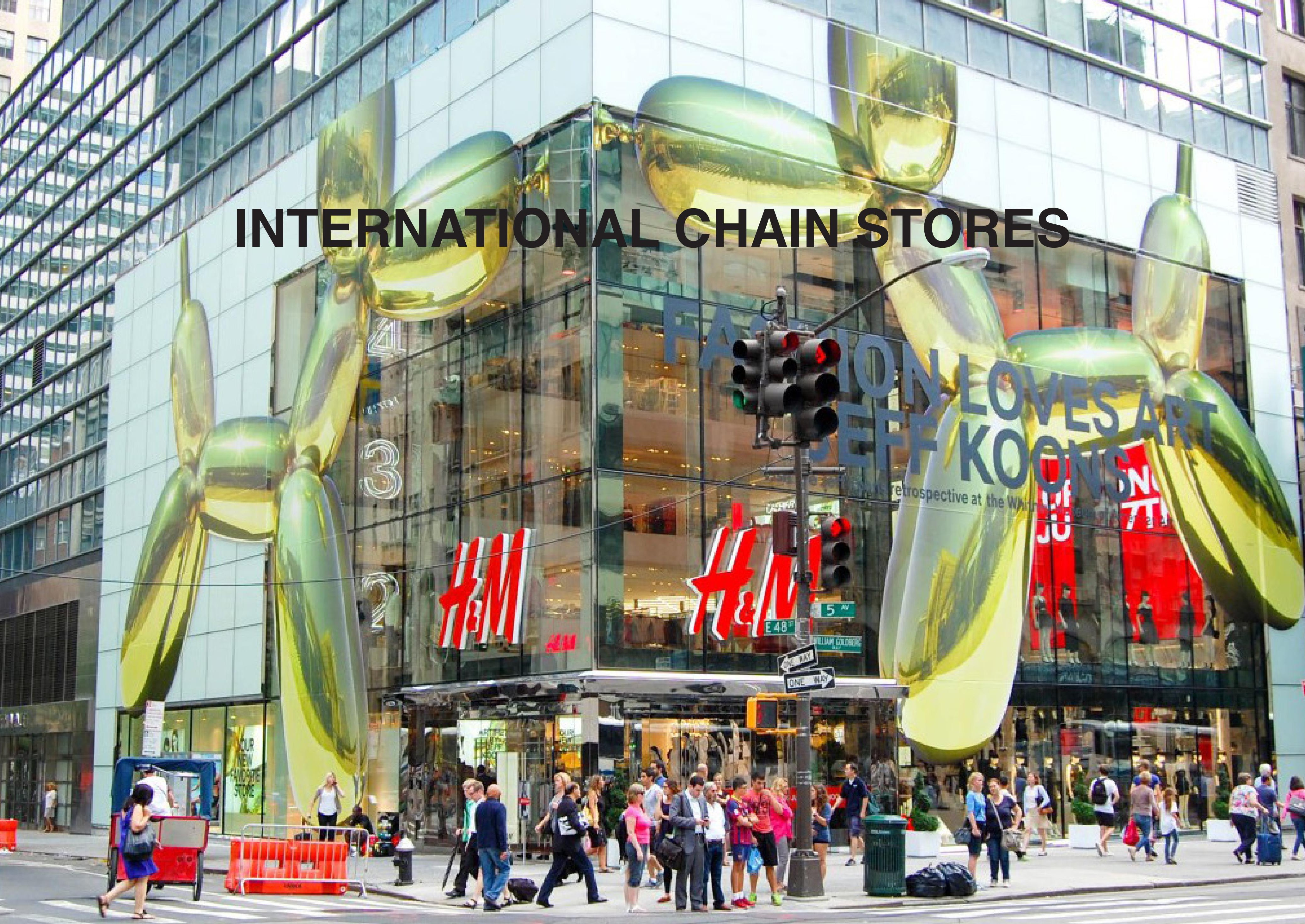


kate spade $\triangle$ NEW YORK

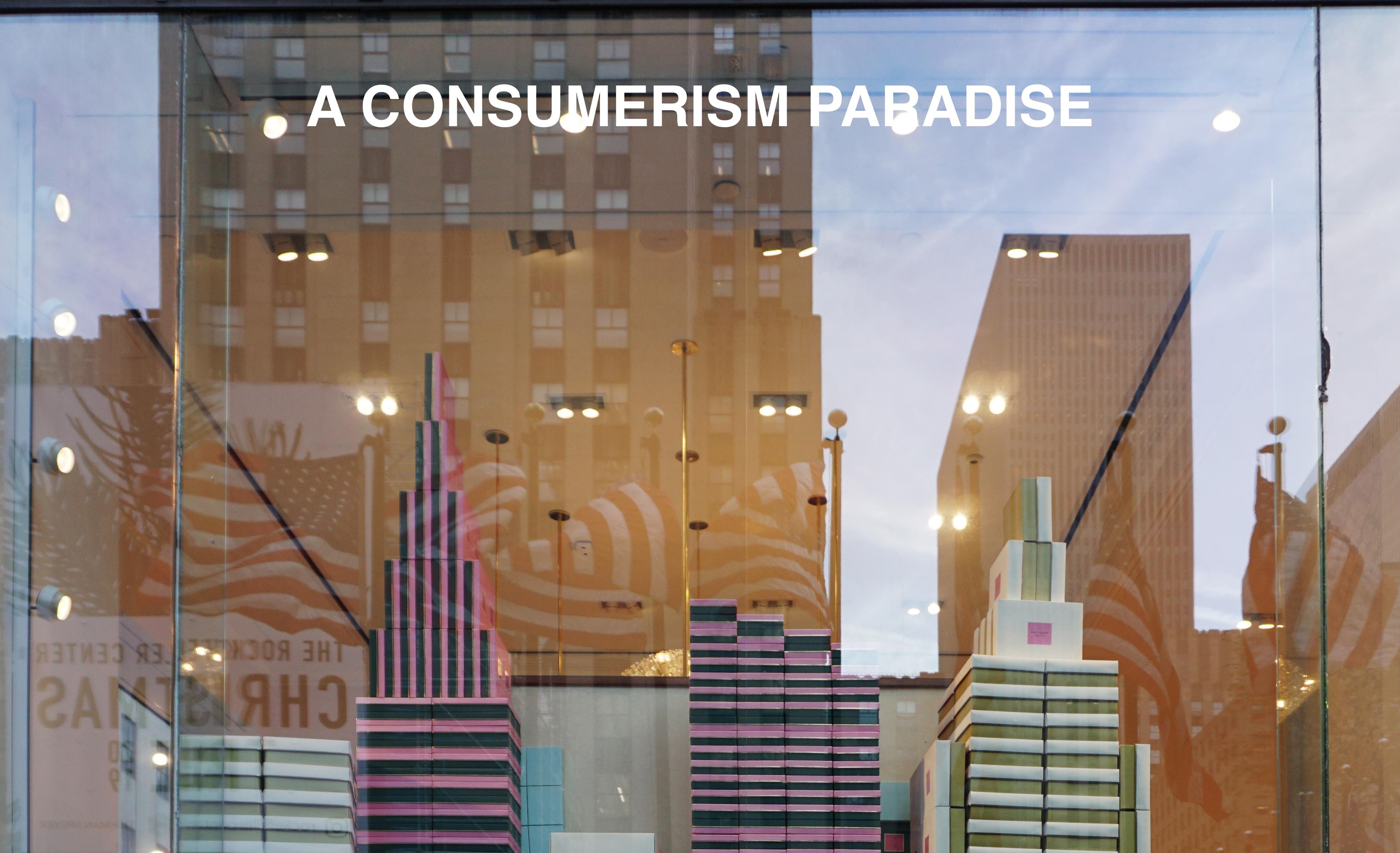




\section{Grocery stores}

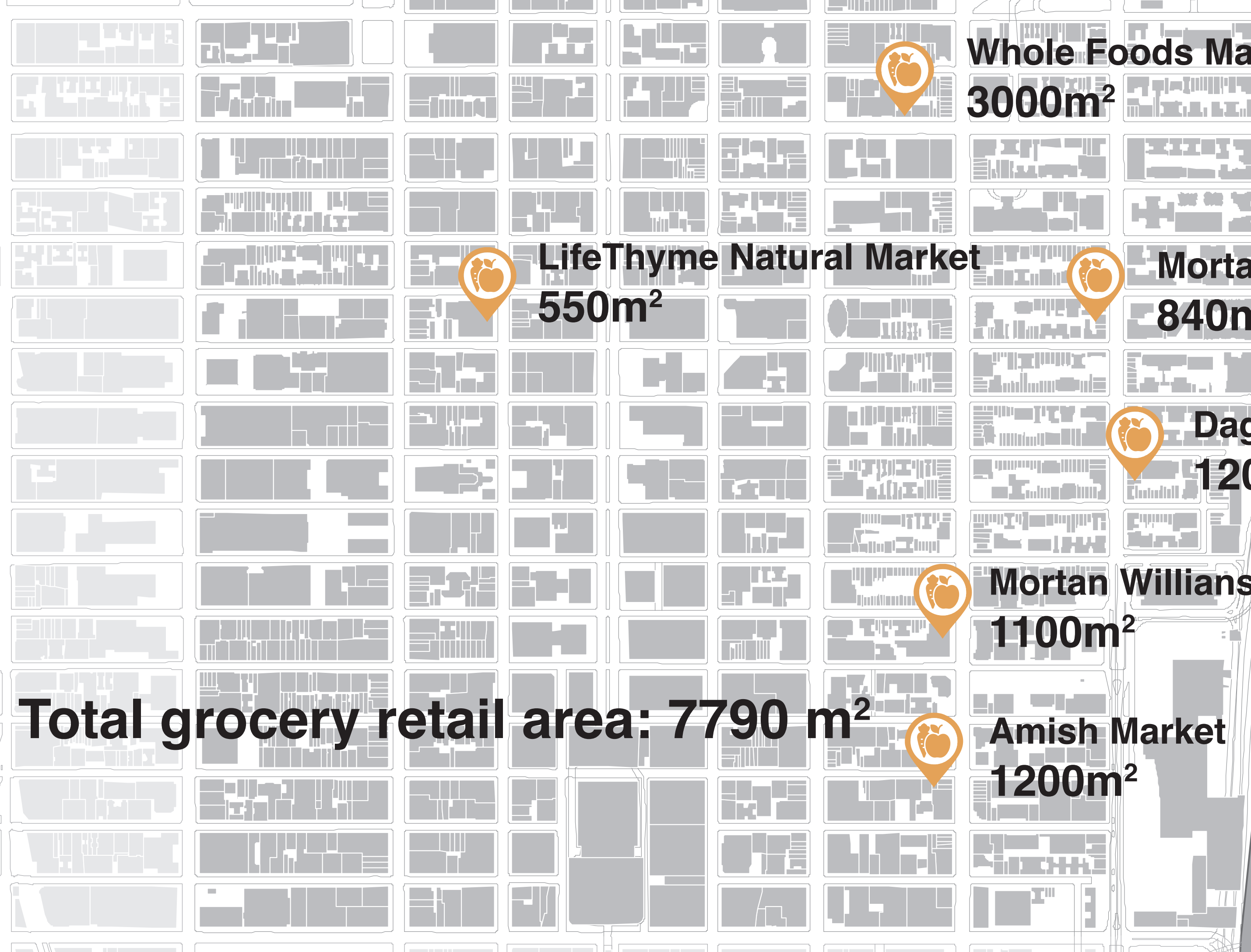

Total grocery retail area: $7790 \mathrm{~m}^{2}$ Amish Market 


\section{Grocery stores to population ratio}

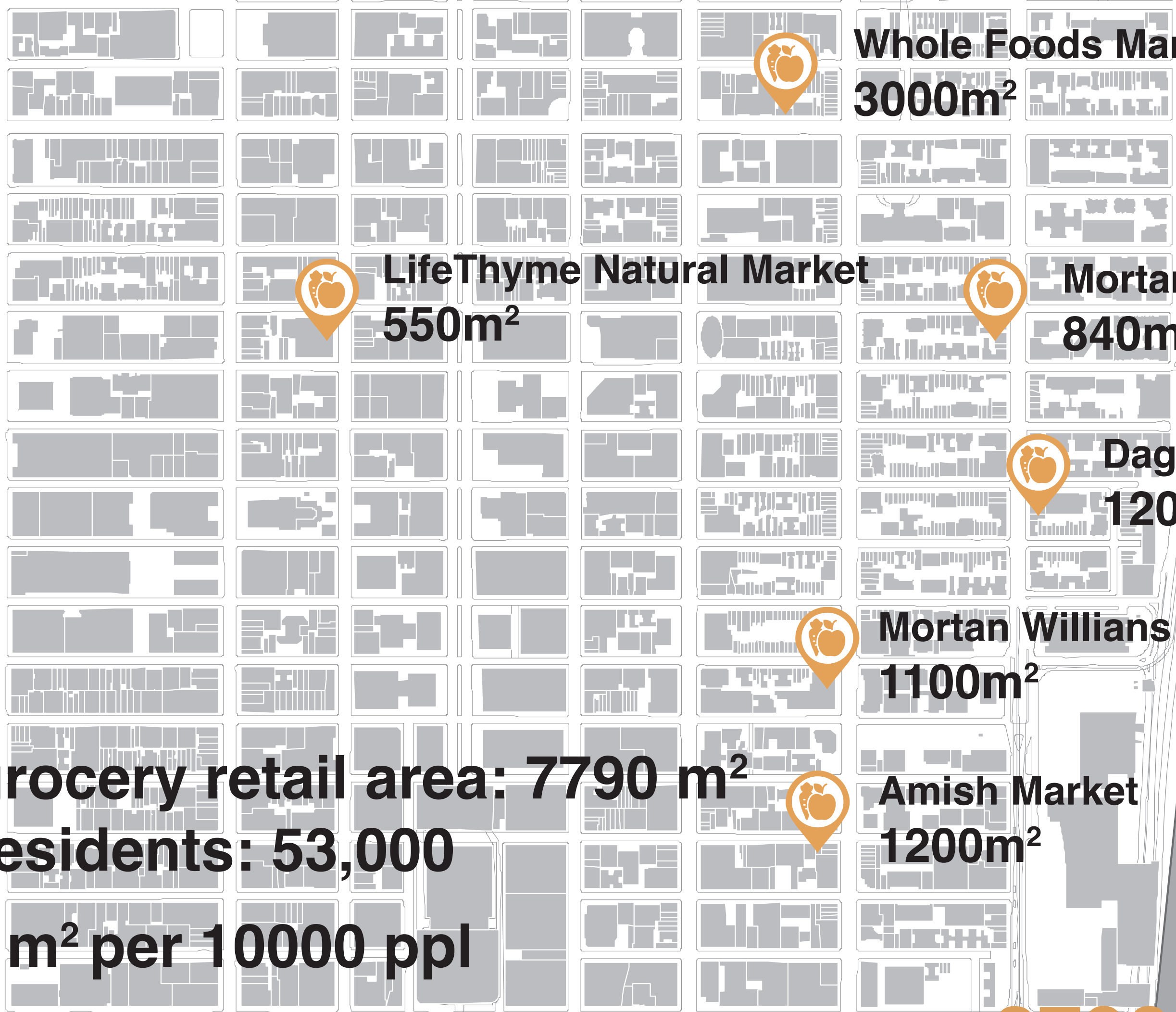

Total grocery retail area: $7790 \mathrm{~m}^{2}$ Amish Market Total residents: 53,000 $1470 \mathrm{~m}^{2}$ per $10000 \mathrm{ppl}$ City Planning Standard Ratio: $\left(\mathbf{3 0}, \mathbf{0 0 0} \mathbf{f t}^{2}\right) 2790$ 


\section{RECREATIONAL SHOPPING}

\section{NECESSARY SHOPPING}
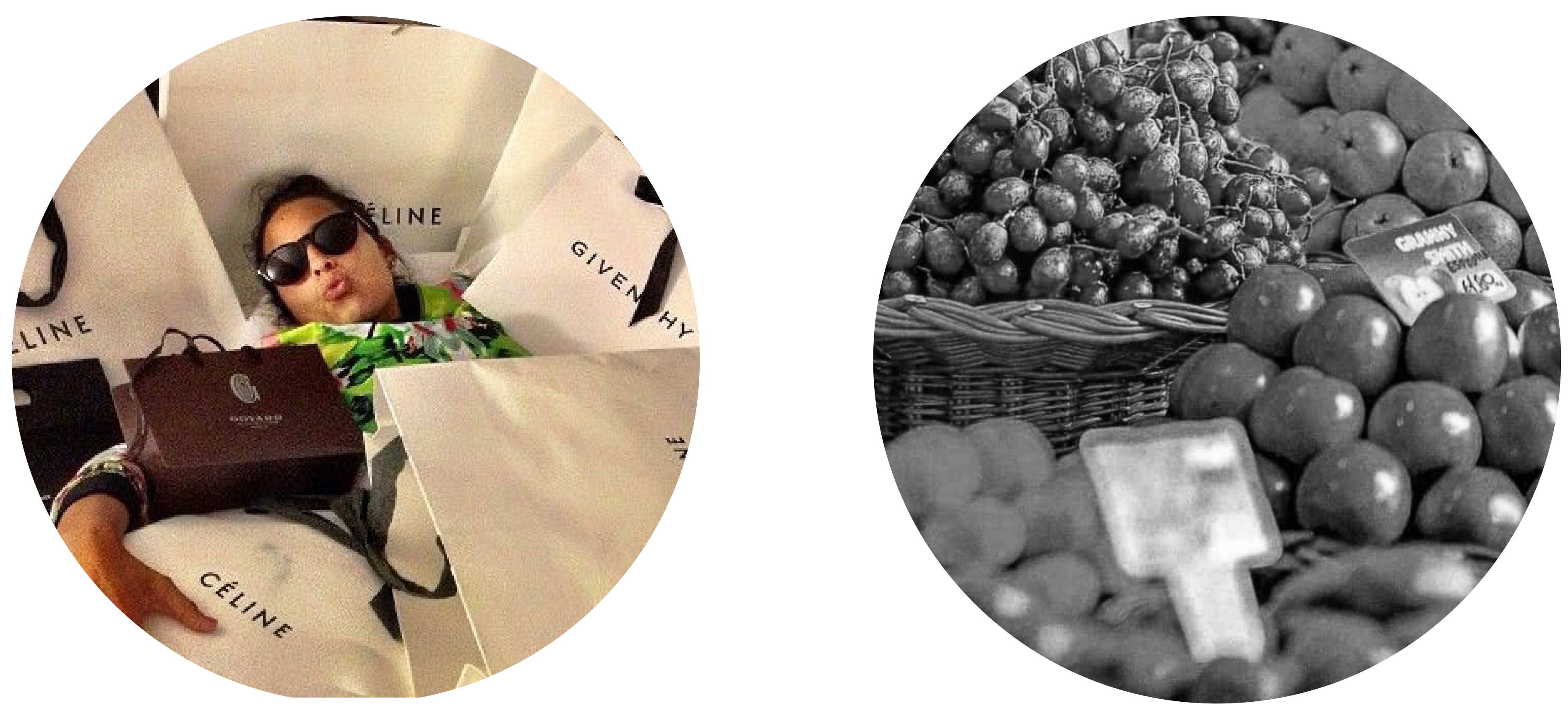


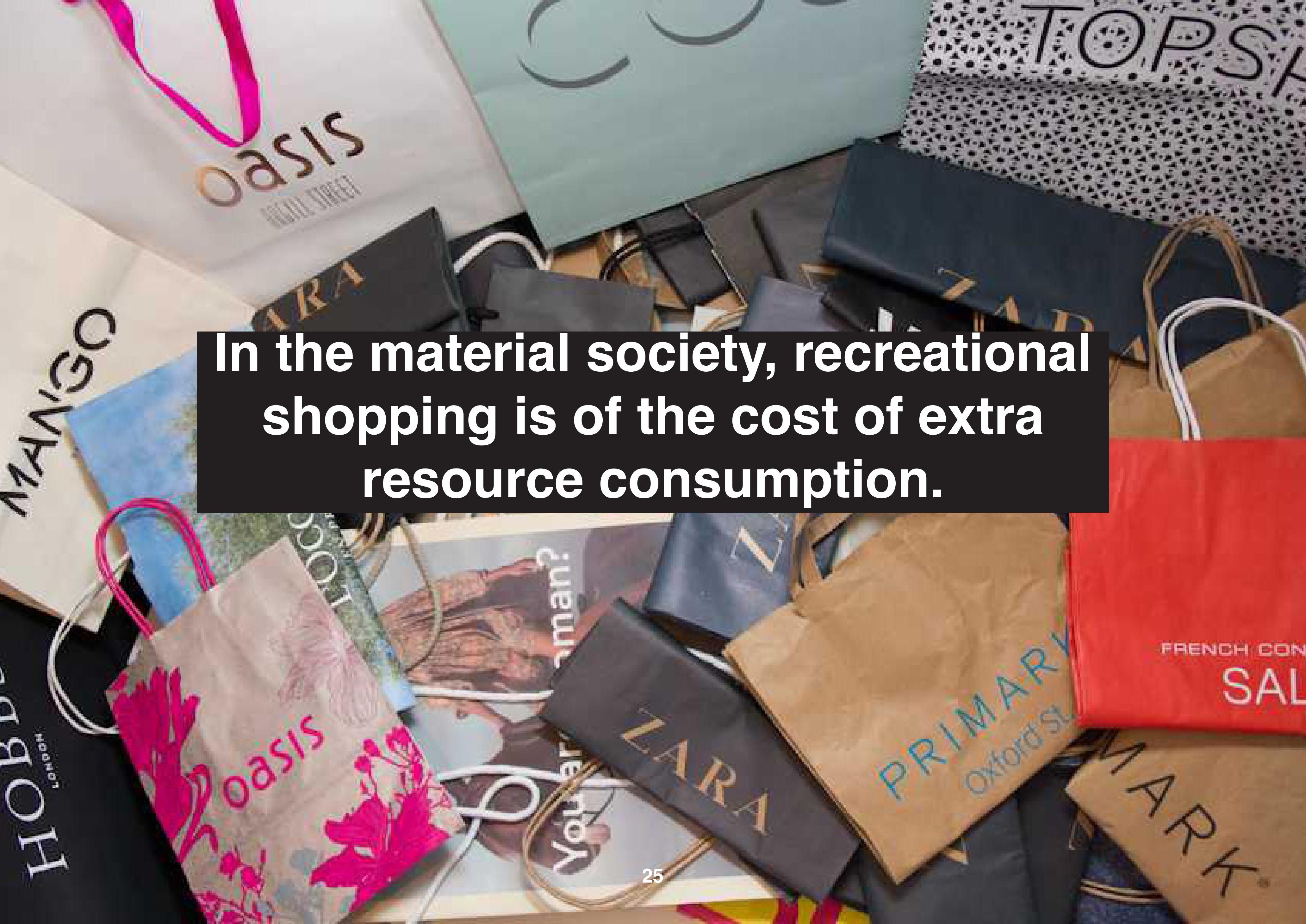




\section{RESEARCH QUESTION}

How to make consumerism socially, economically and environmentally sustainable?

Can necessary shopping replace recreational shopping as an enjoyable experience? 


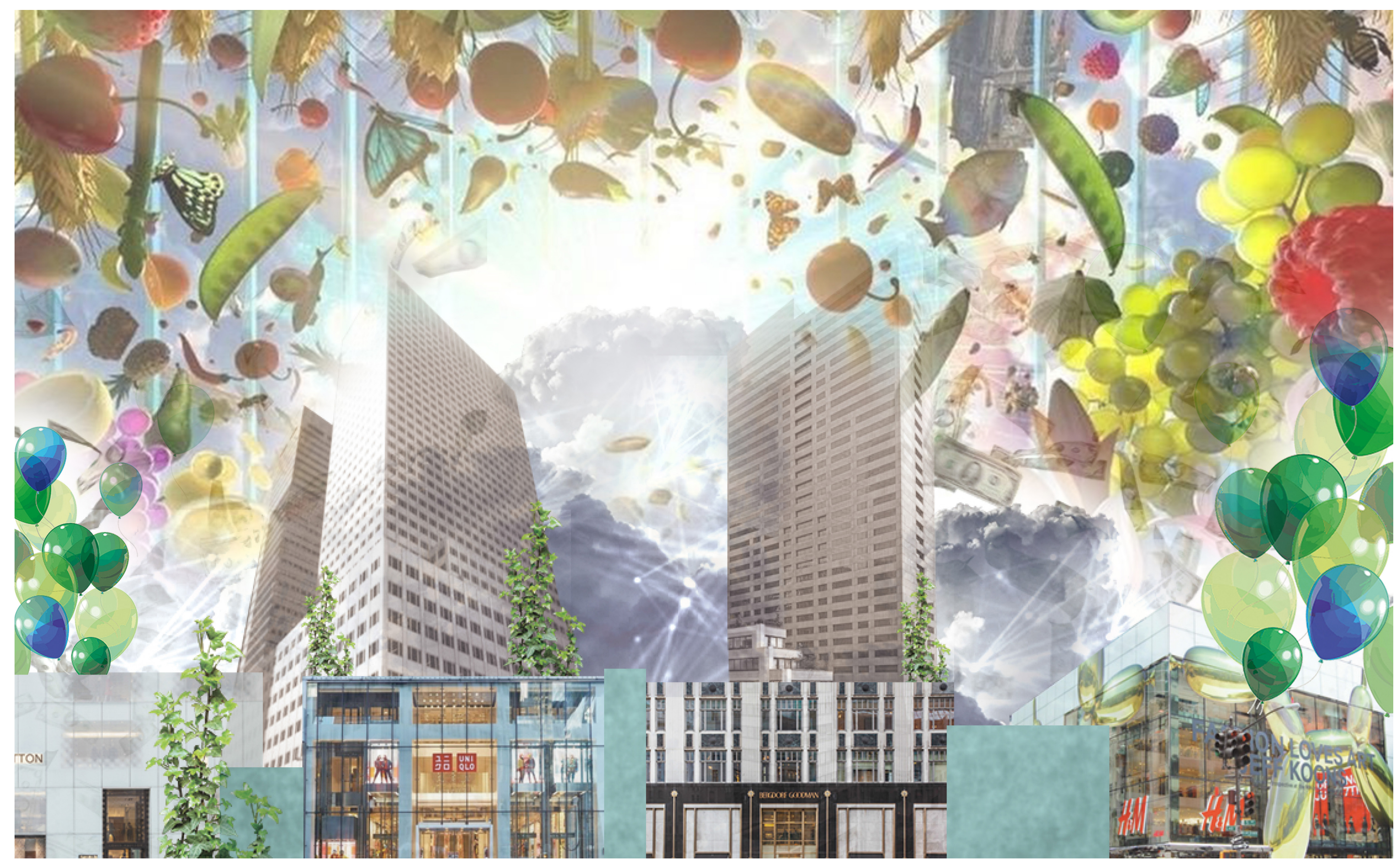




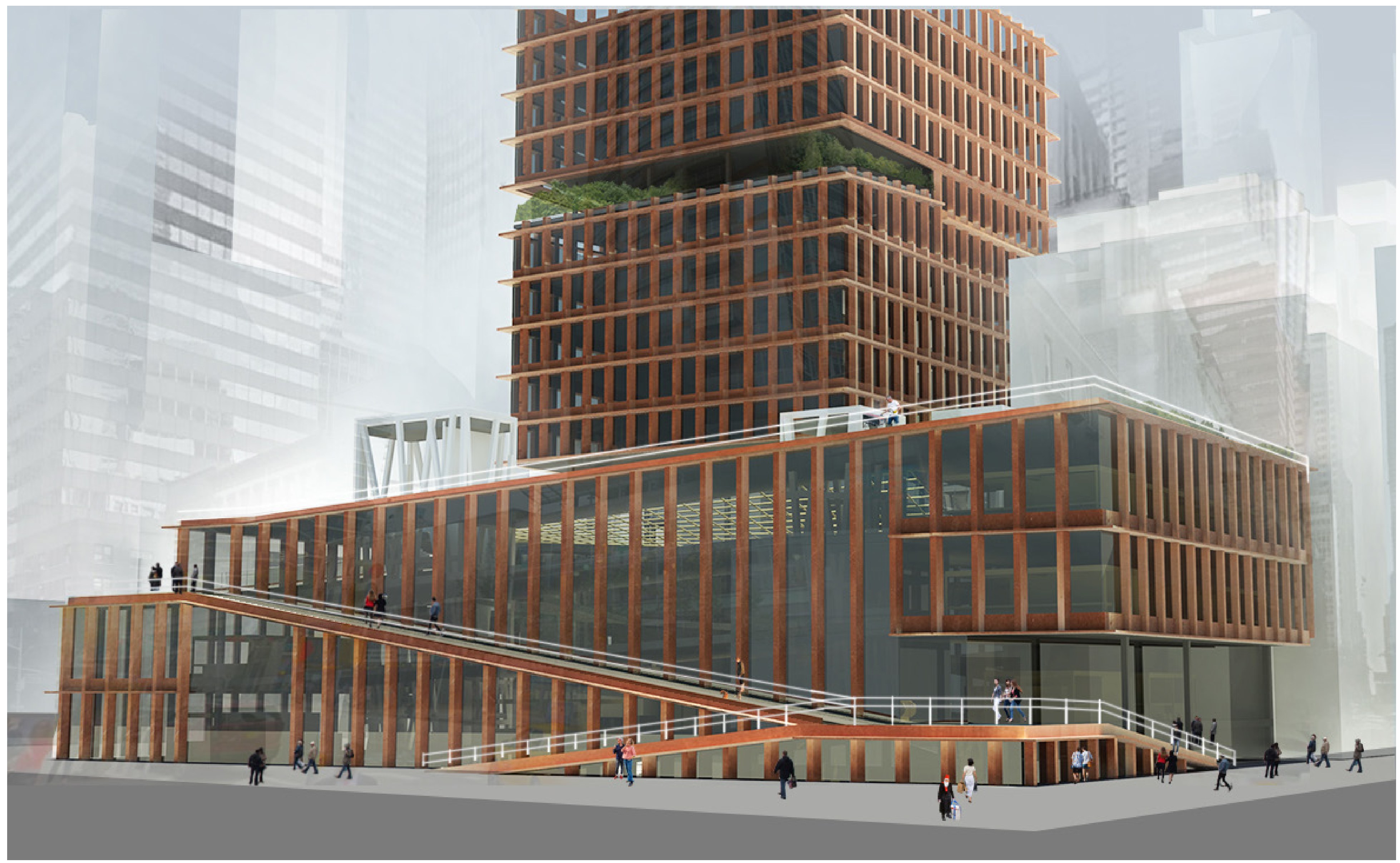




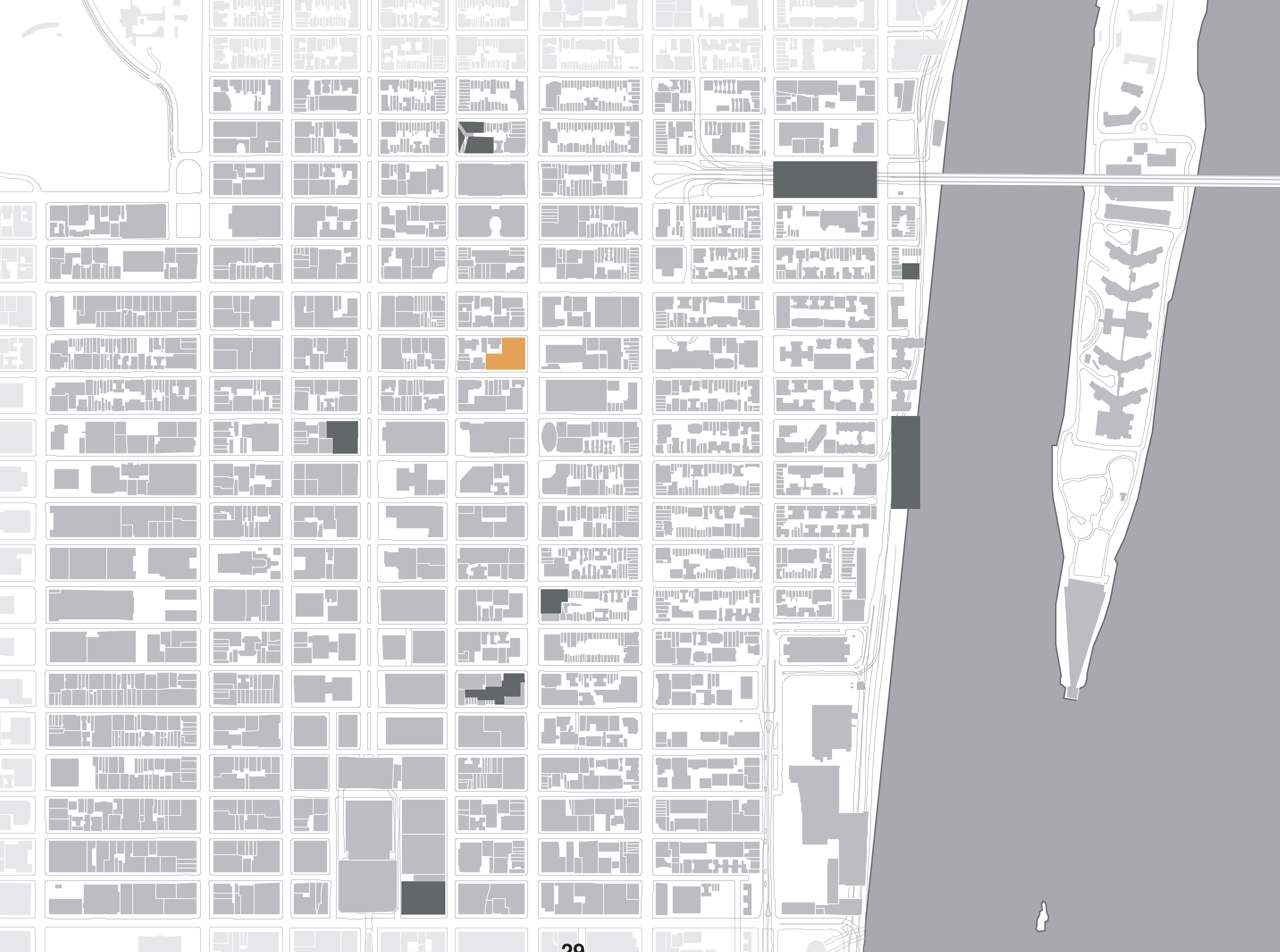




\section{People}

Who is shopping what here? 


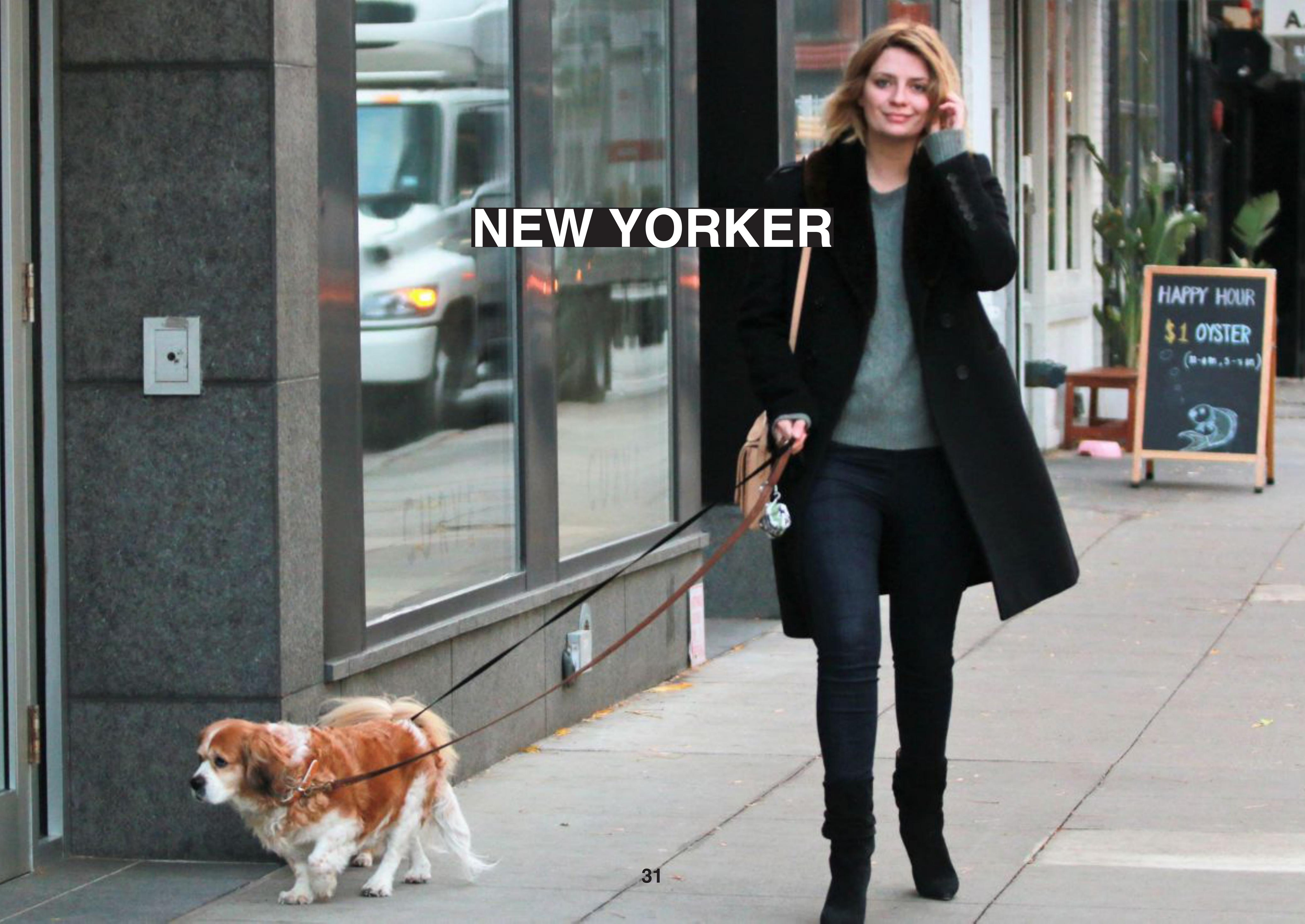




\section{New Yorker's Proffle}

-High income, high purchase power -Ethnically diverse

-Lack of grocery stores for buying fresh food

-Lack of gathering space which is crucial to build sense of community

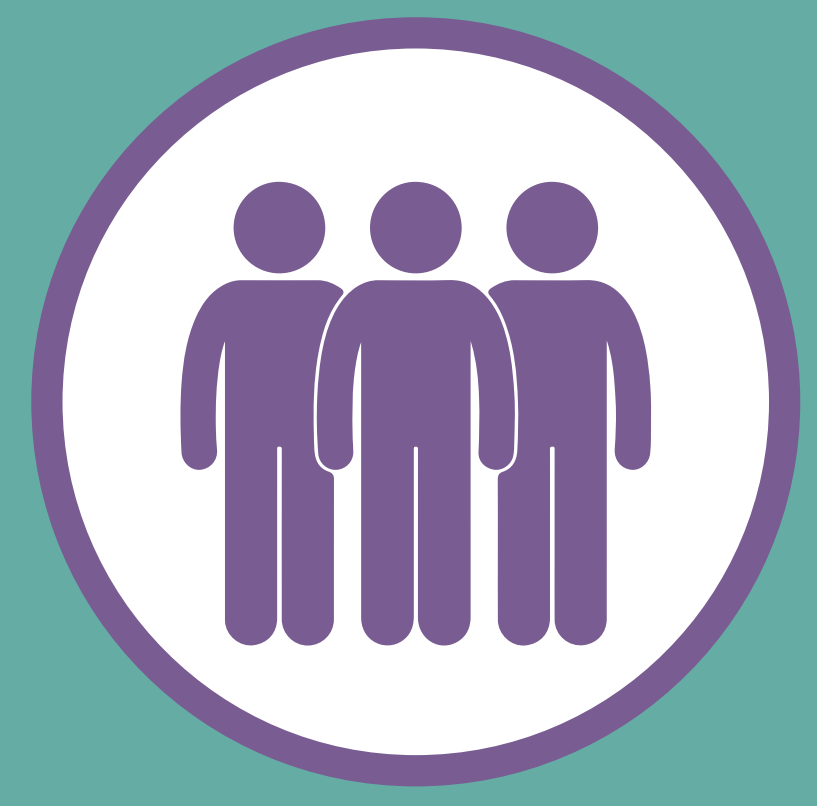




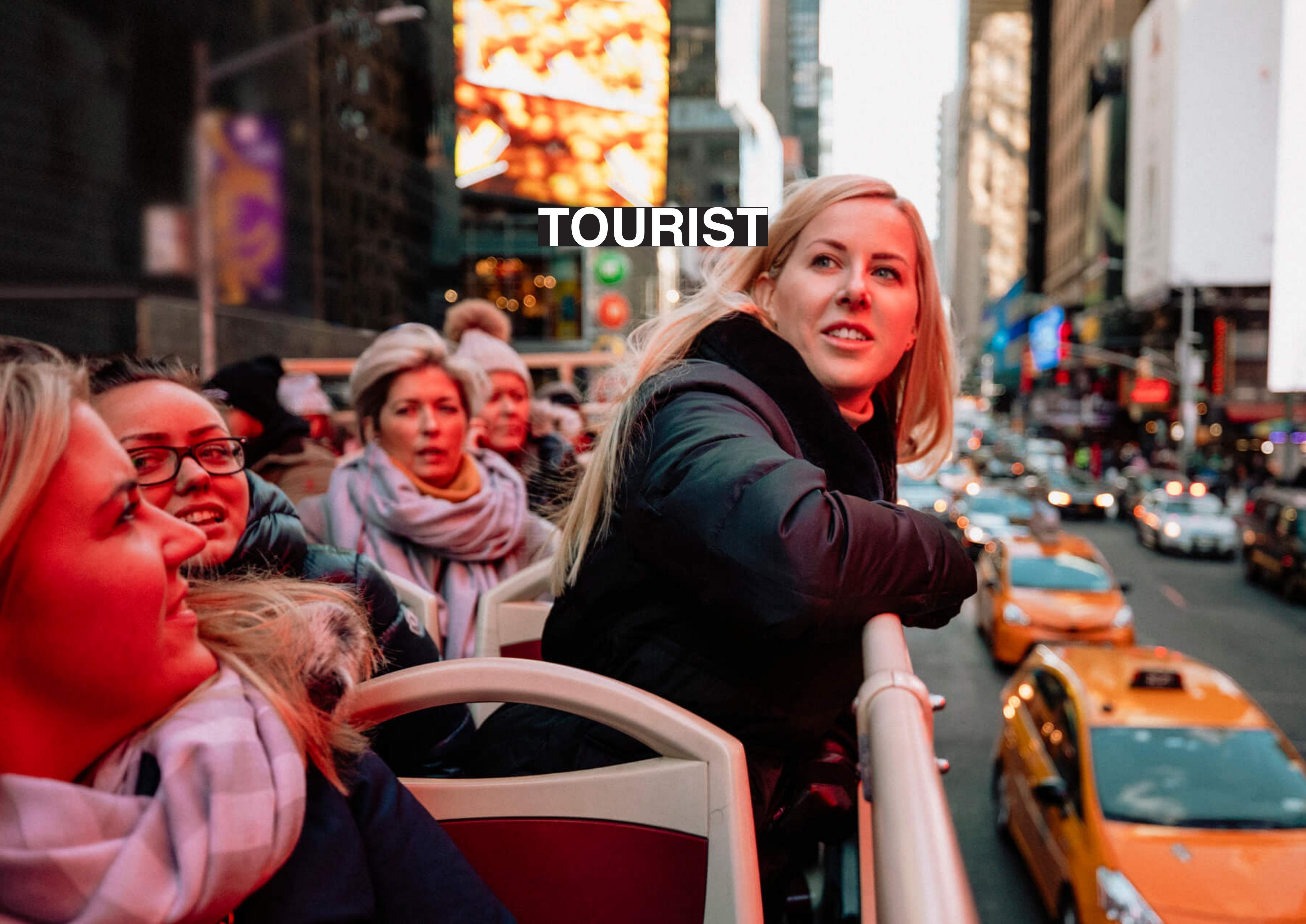




\section{Tourism as one of the leading sector in NYC}
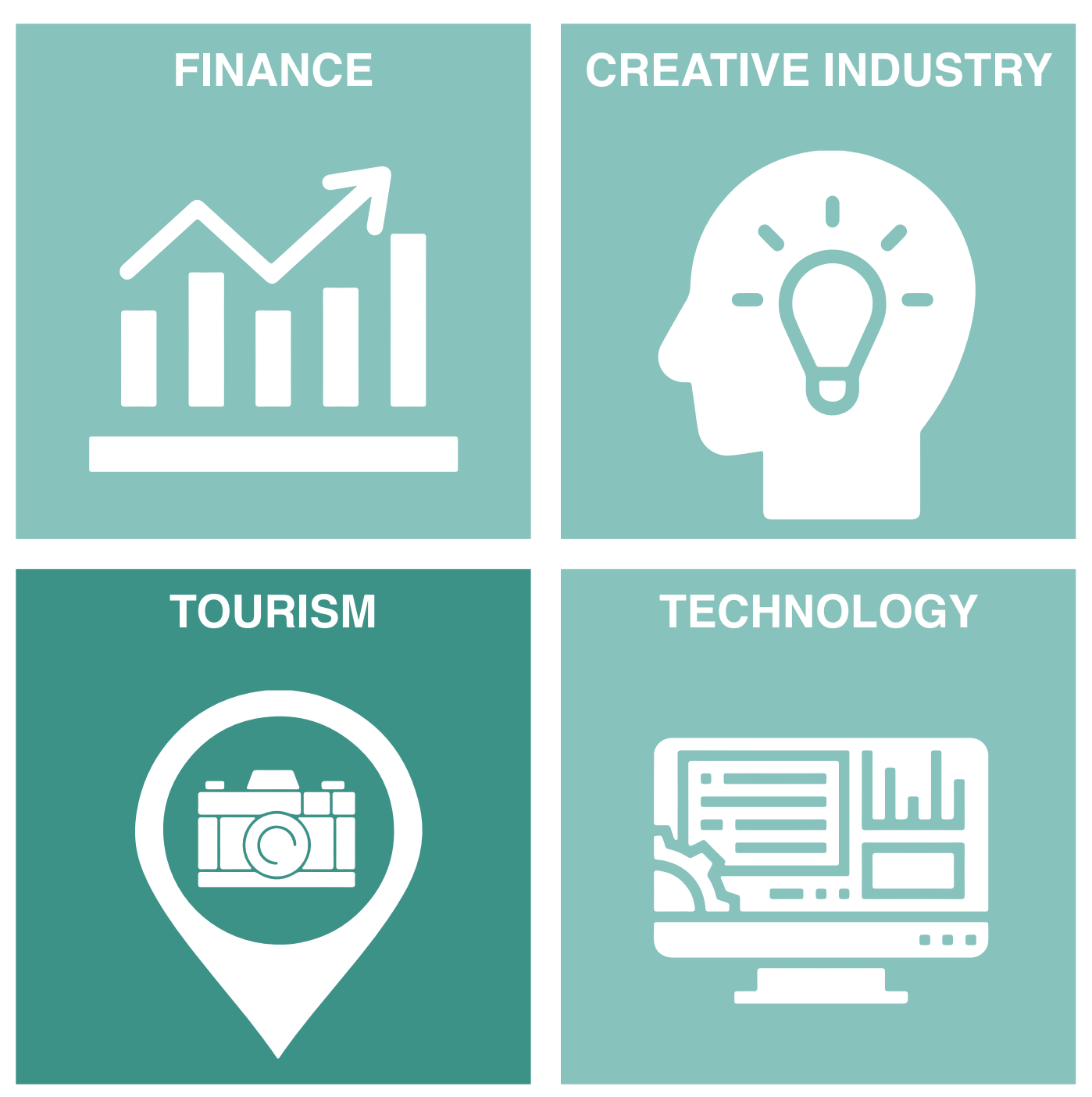


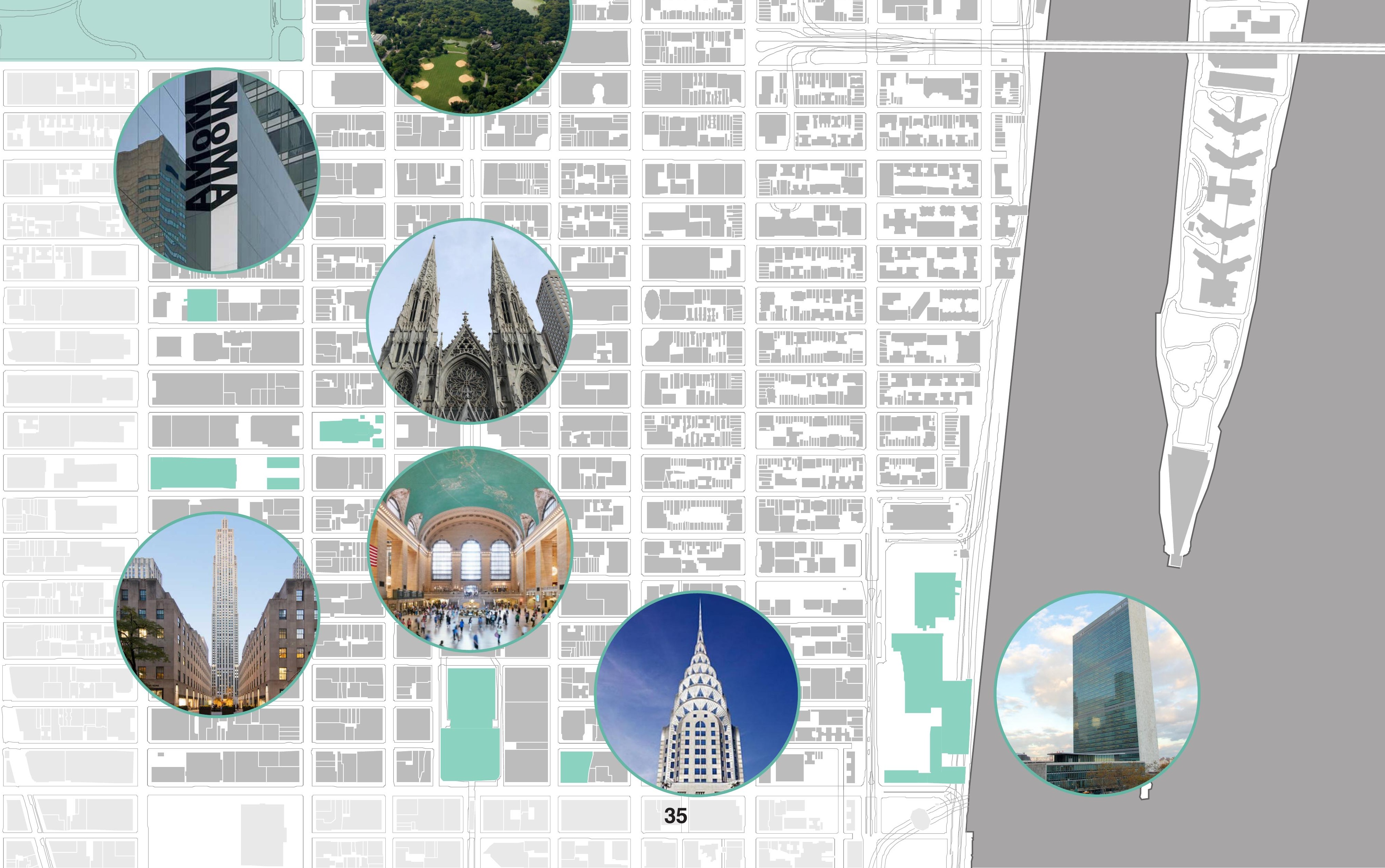




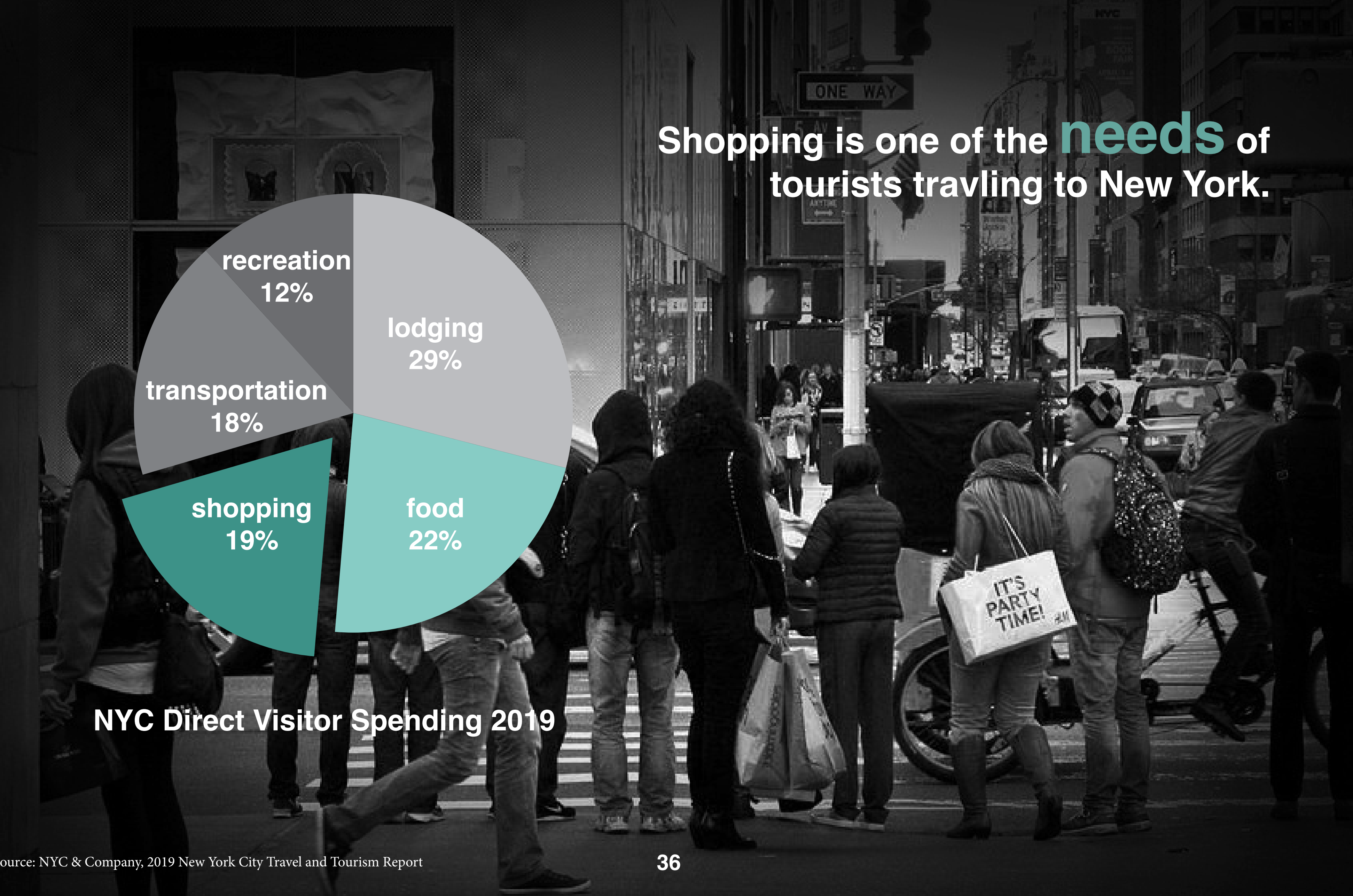




\section{Retails on Fifth Avenue}

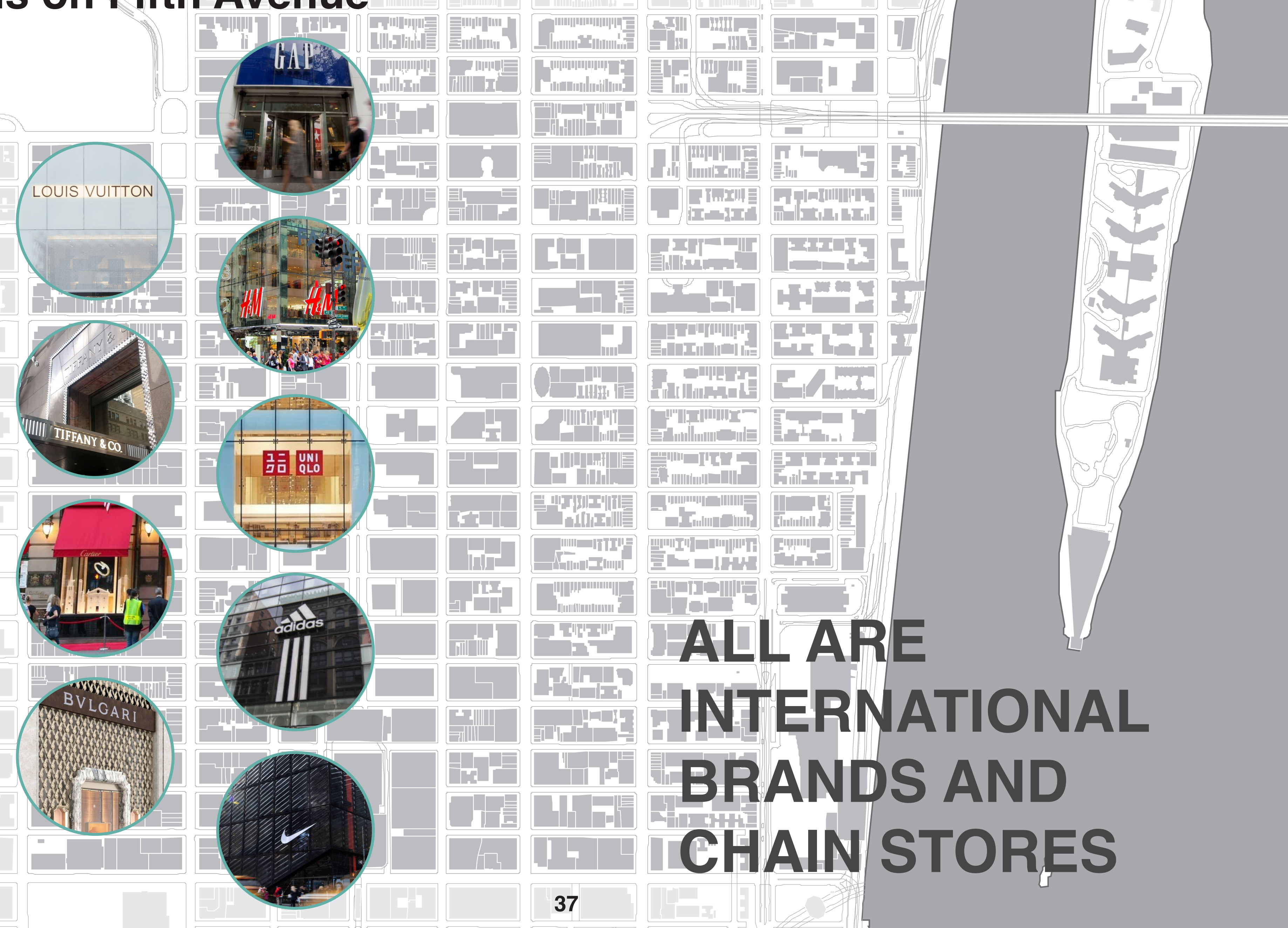




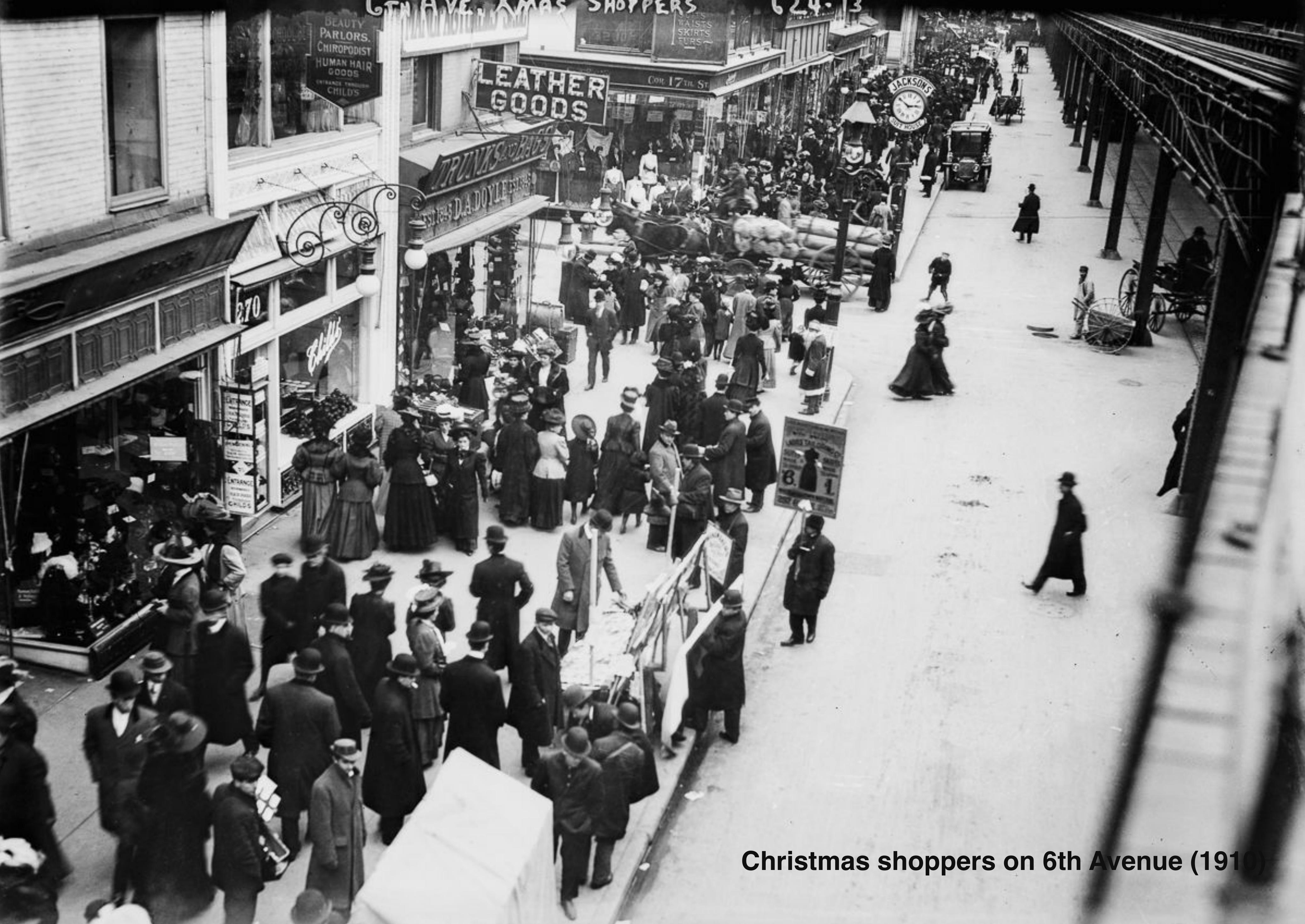




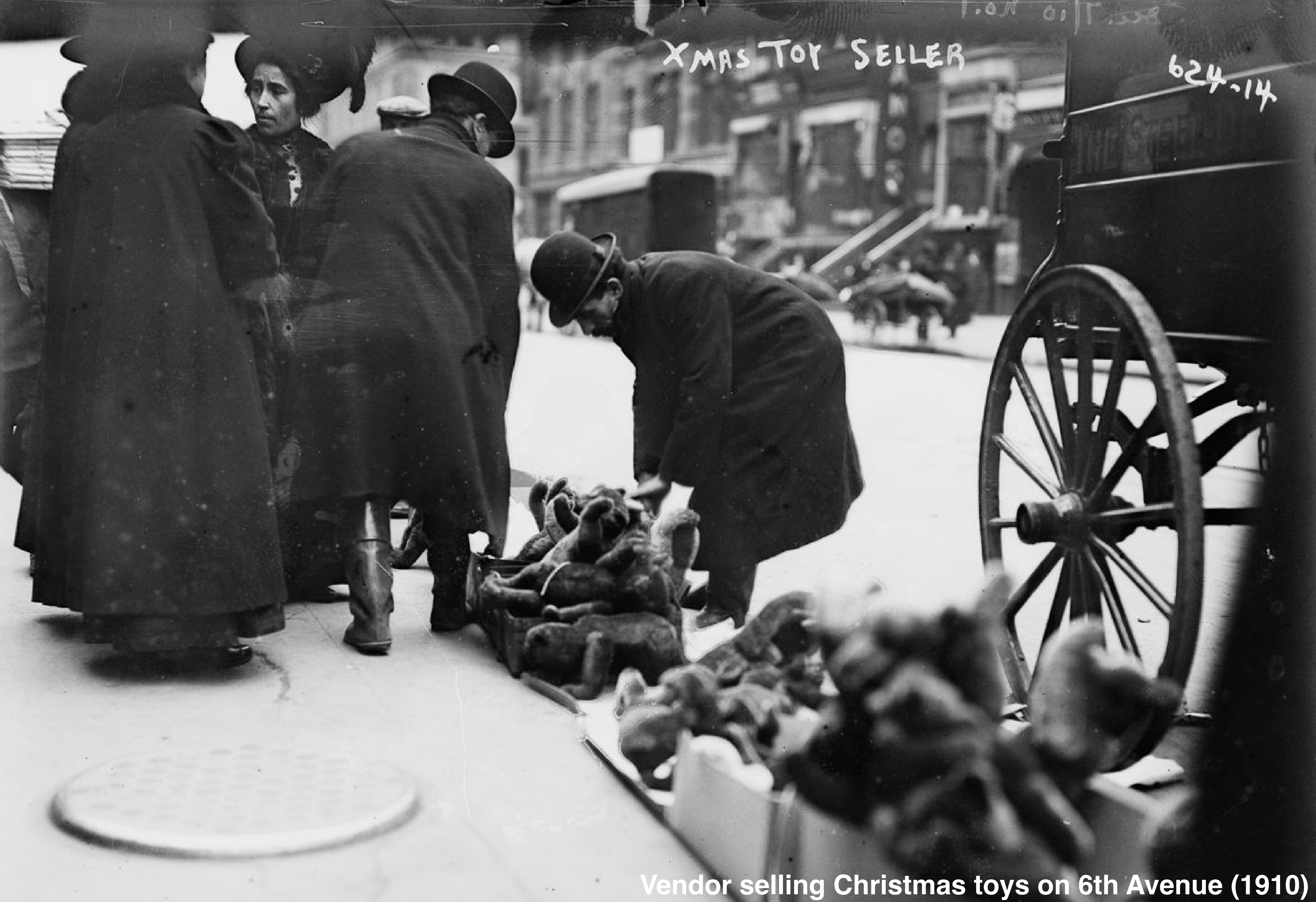




\section{NYC Tourists Proffle}

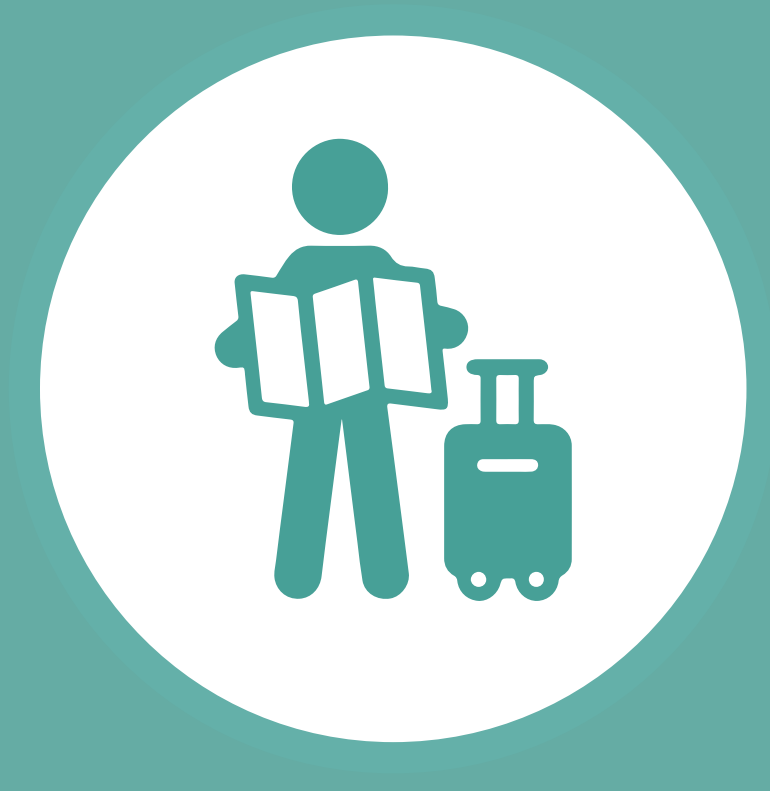

-High population, high purchase power -Shopping as a need

-Lack of featured shopping experience that is authentically 'New York'

-Unsustainable consumption from excessive unecessary shopping 

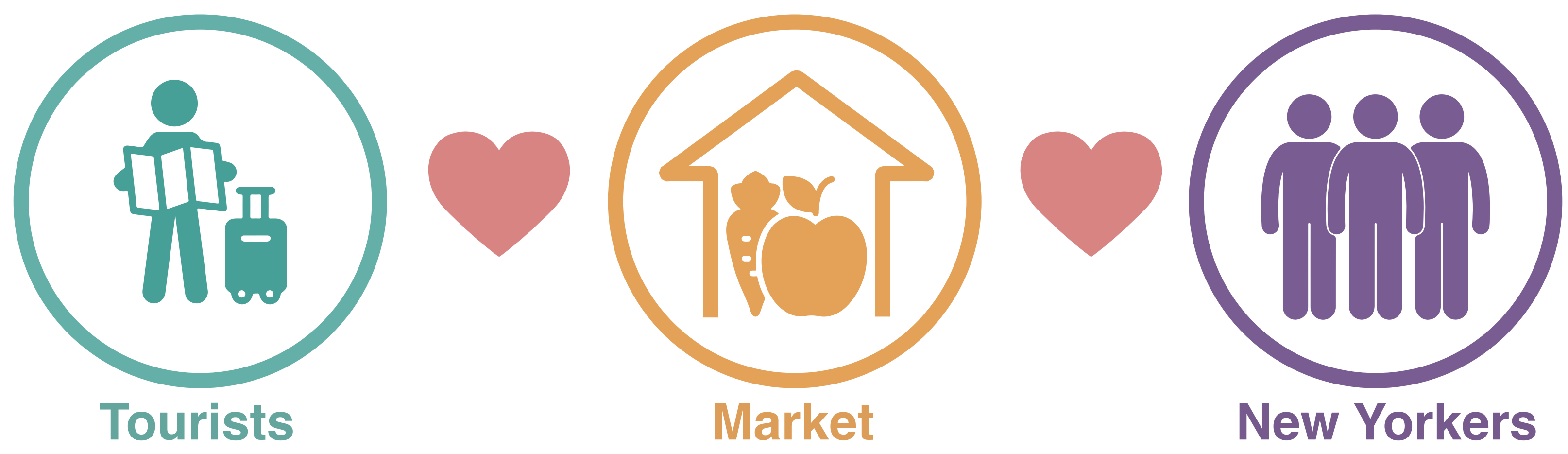


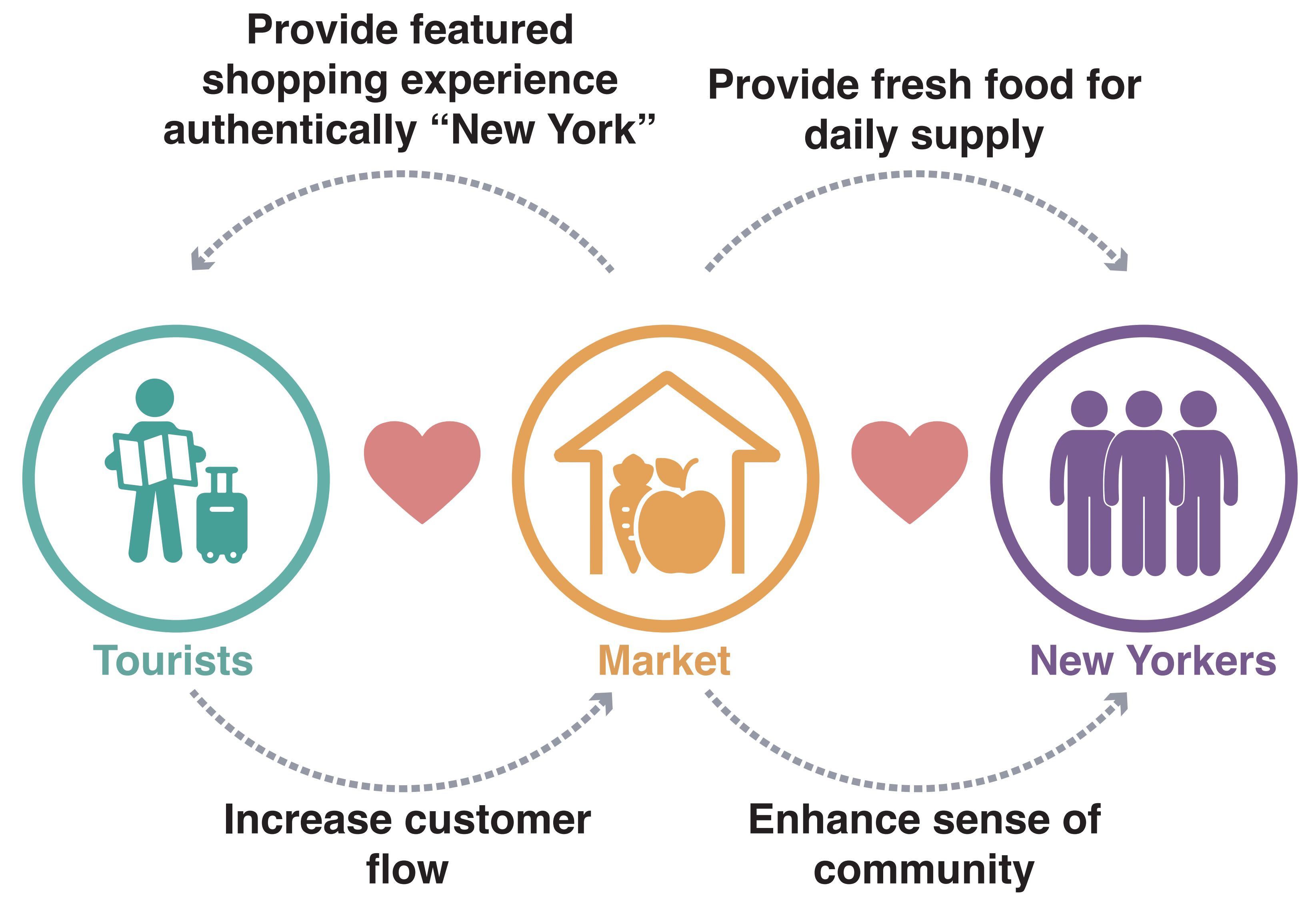




\section{Site}


(1)

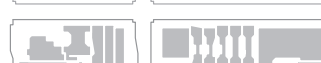

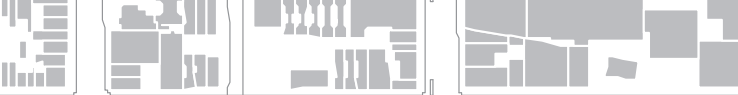

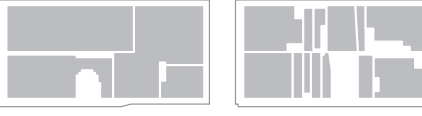

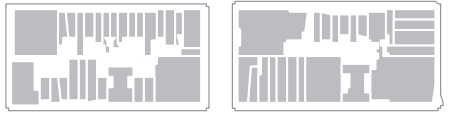

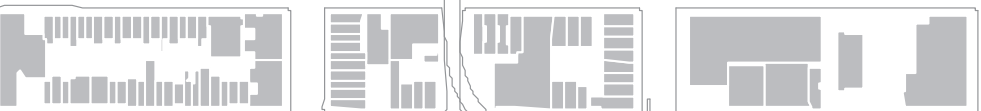

늘

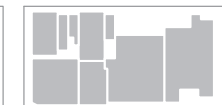

데

VALLEY OF GIANTS

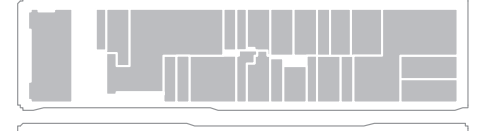

Gin|

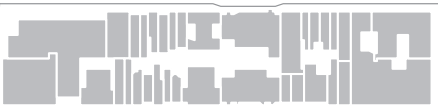

in in

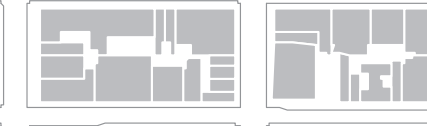

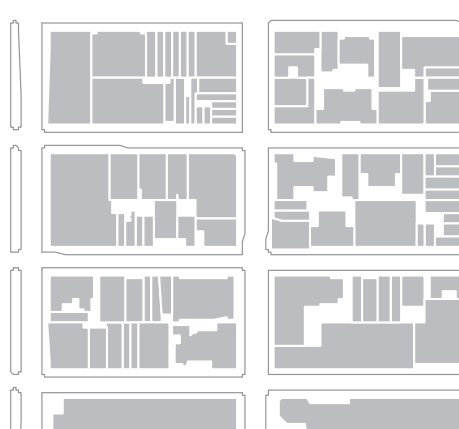

다다

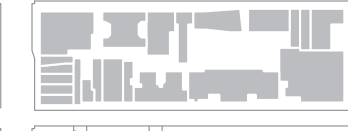

$2+1+35$

Tlisen

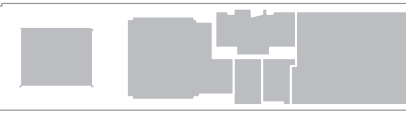

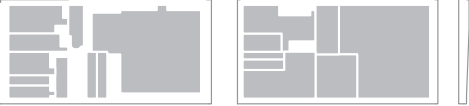

배내

대는도

드는

senthe

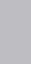
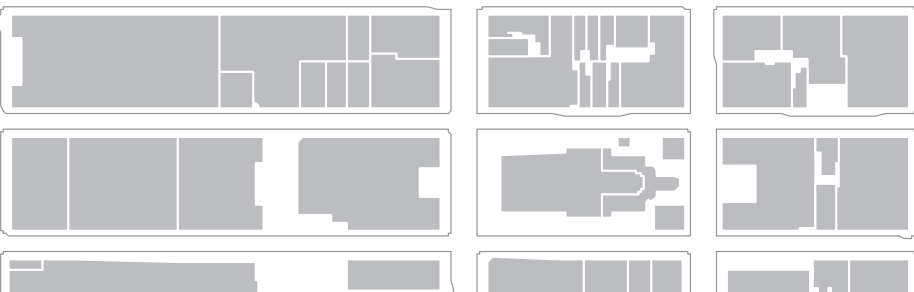

-

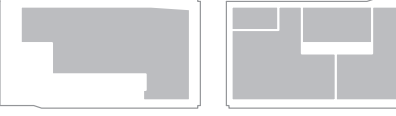

(1)

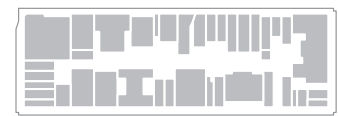

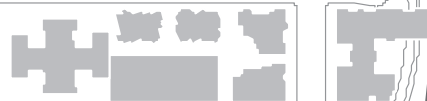

$\square+2$

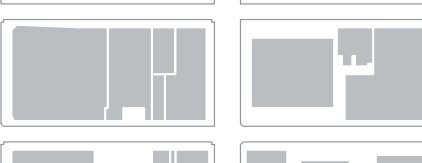

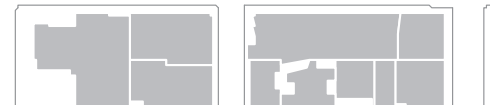

$\square$

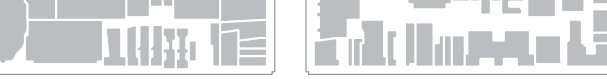

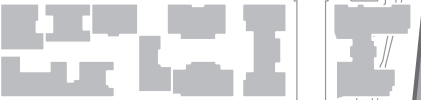

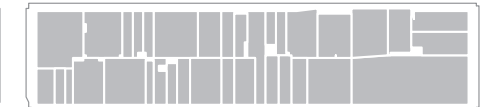

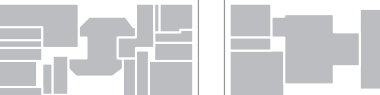

口十

rerinin:

(1)

E r ing

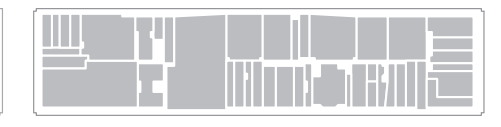

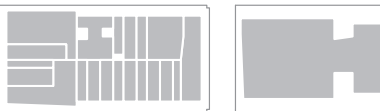

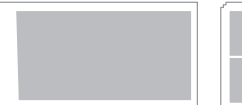

Finitint

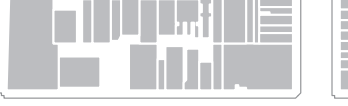

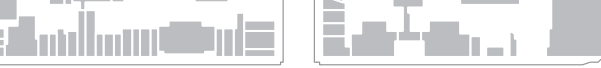

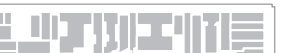

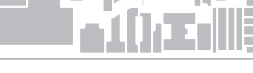

Fim

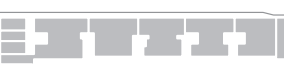

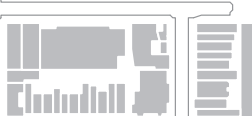

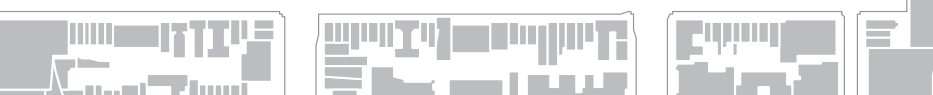

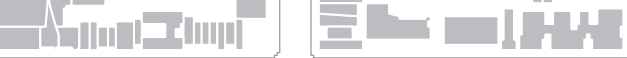

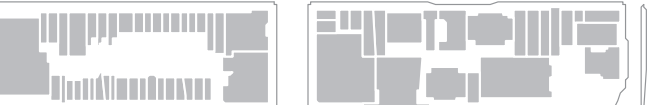

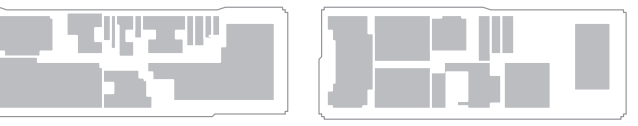

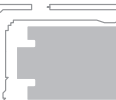

$\square \square \square$

$\square$ Din

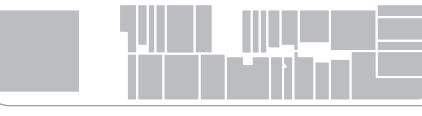

(I)

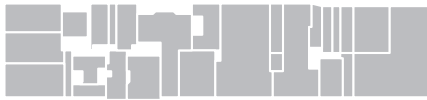

alin] EL

$[\sqrt{11}-1$

IIII $\square$

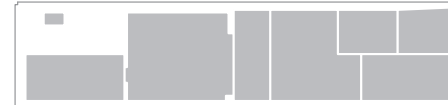

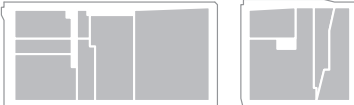

(x)

III

貫

1.4 पII" 
Fifth Avenue

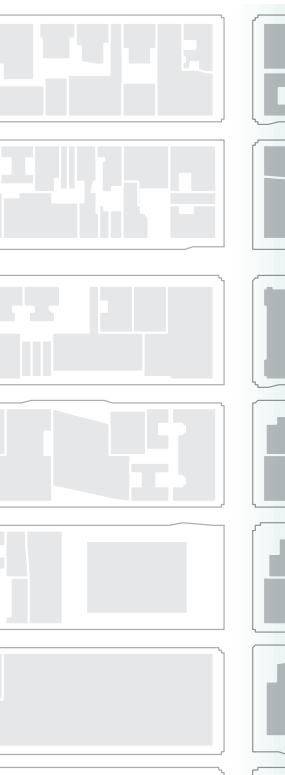

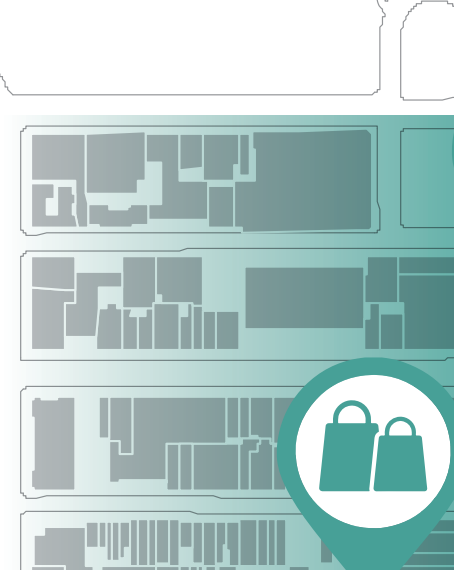

(a)

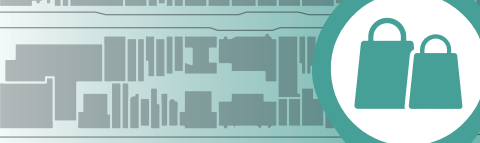

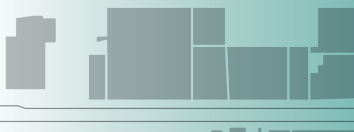

\section{in}

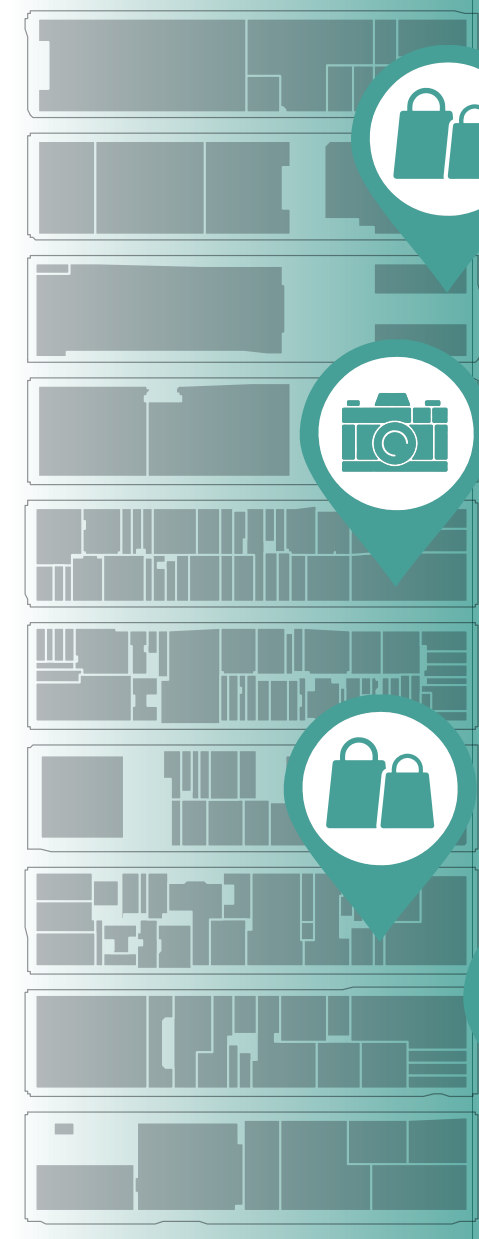

(6)

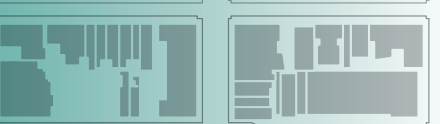

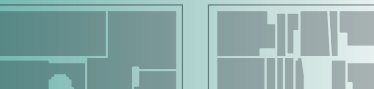

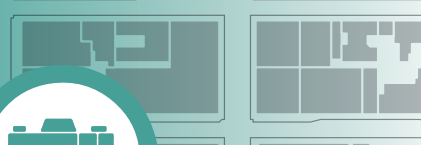

(a)

rit

(1IIIT)

(ia)

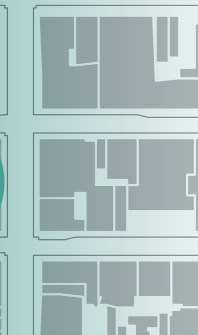

atirit

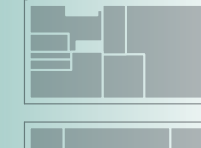

\section{it엑}

(10)

(10)

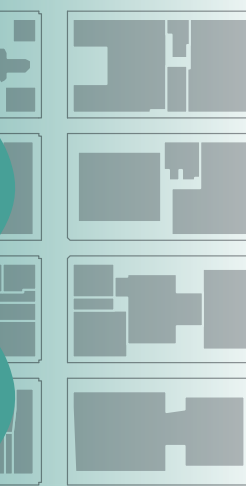

$\frac{1}{14}$
$\square-4$
$\square-4$

$-7-4$
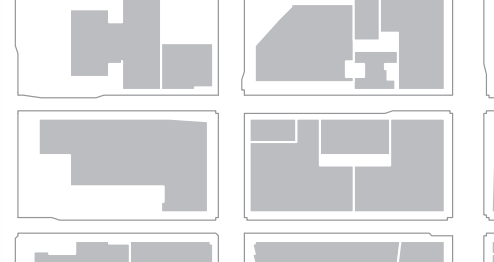

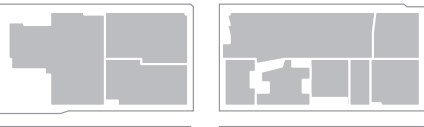

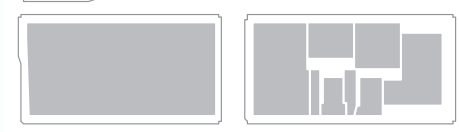

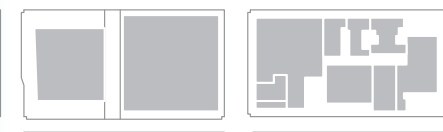
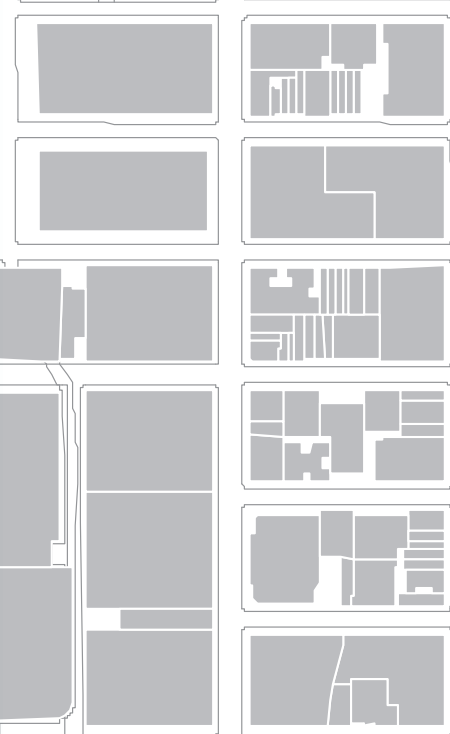

$-$

Lin|

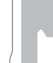

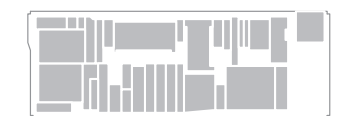

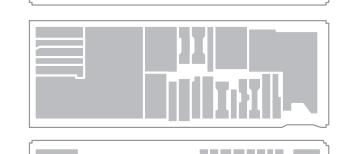

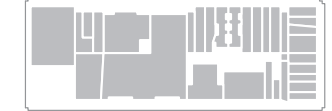

대든

다는

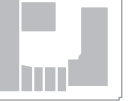

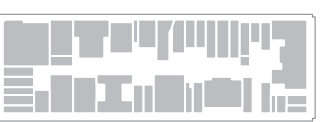

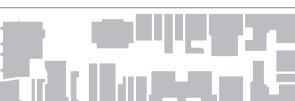

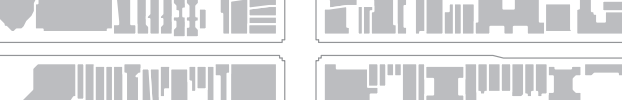

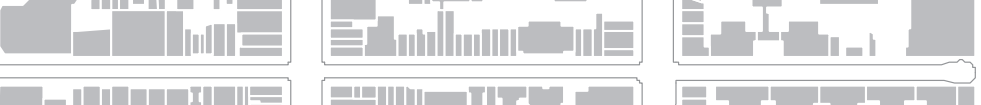

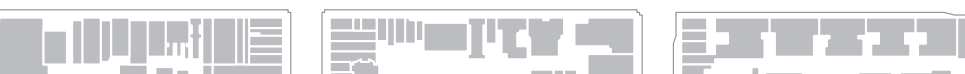

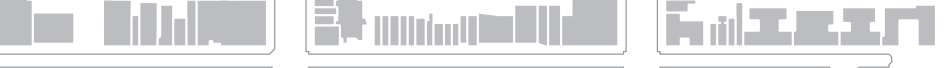

势

(5)

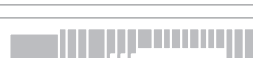

(lith

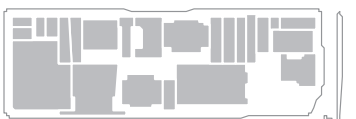

Fy

.

Fillinin

(n)

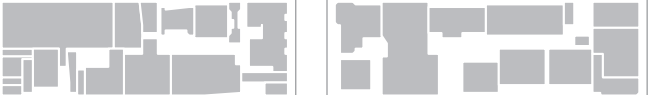

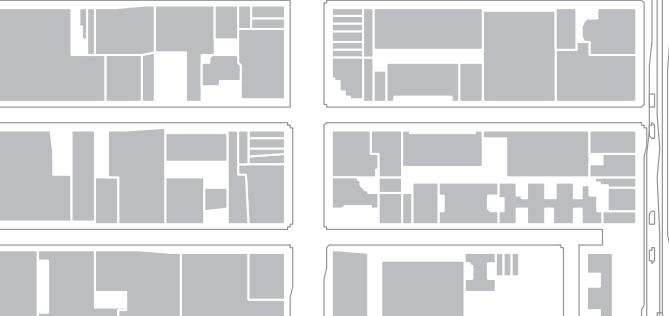

1. 


\section{Fifth Avenue - now}

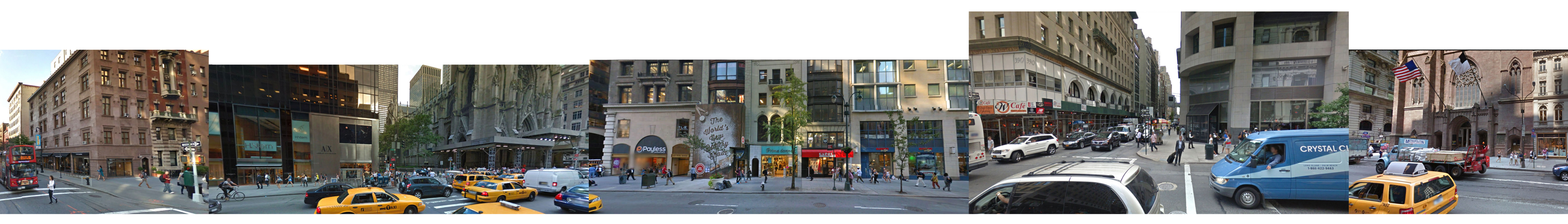

"The most expensive and elegant street in the world." -Forbes magazine, 2018 


\section{Residential District}

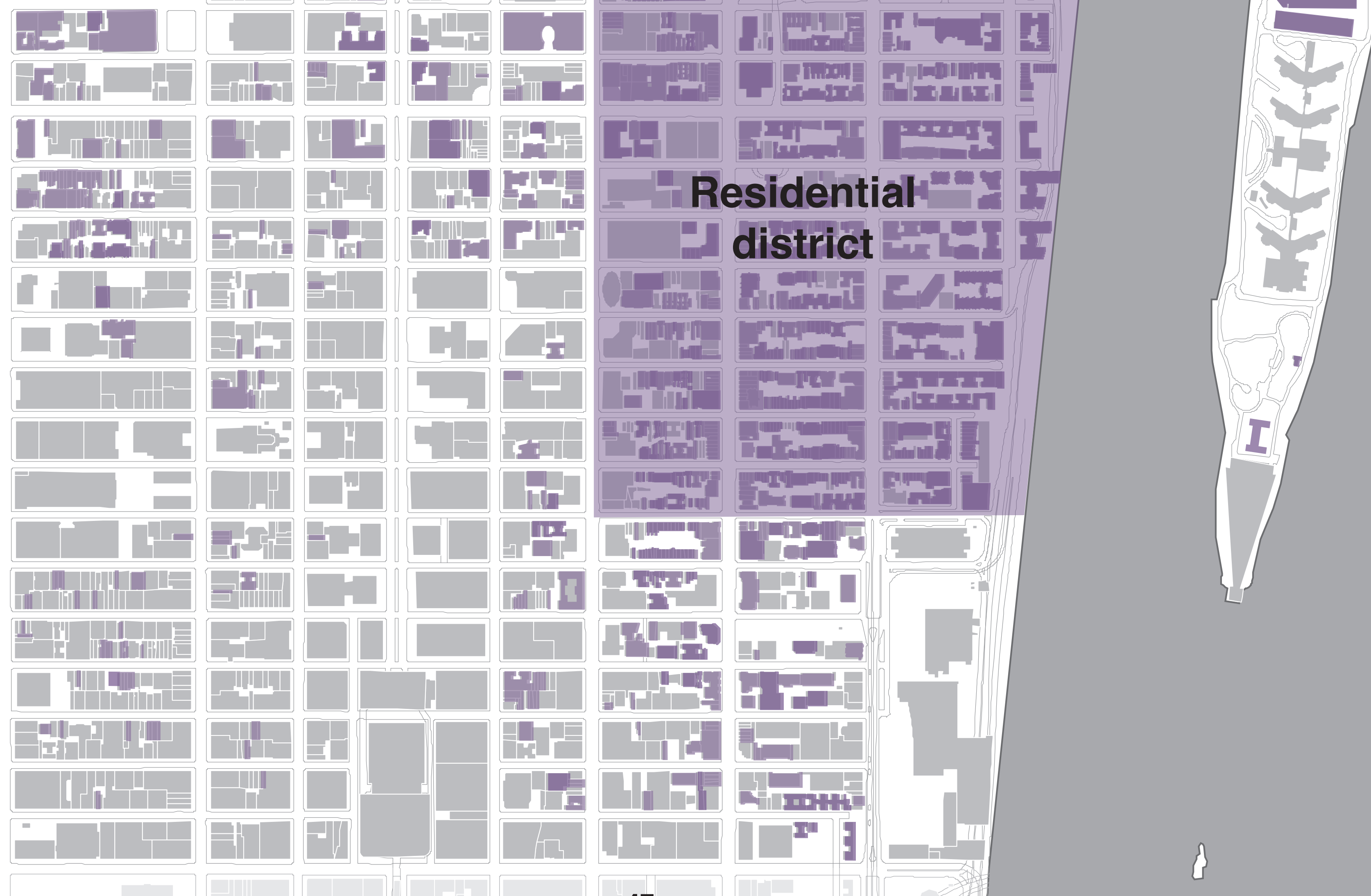

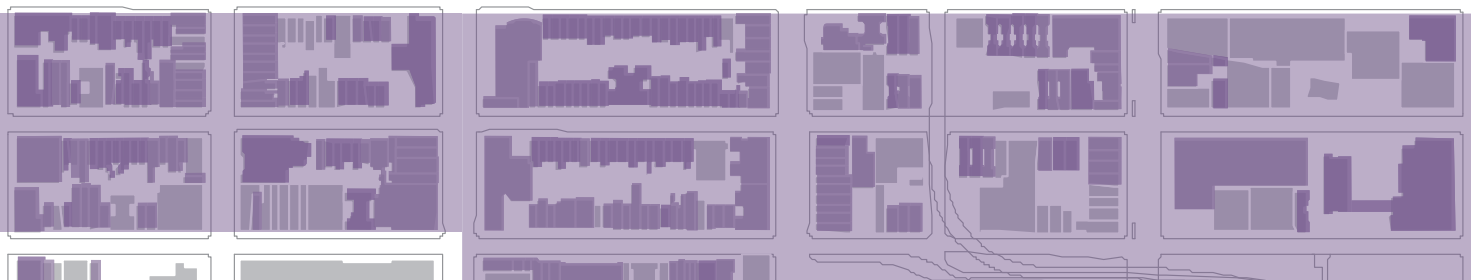

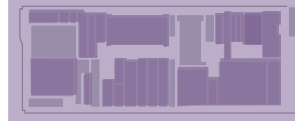

(II)

(1) 


\section{The buffer zone}

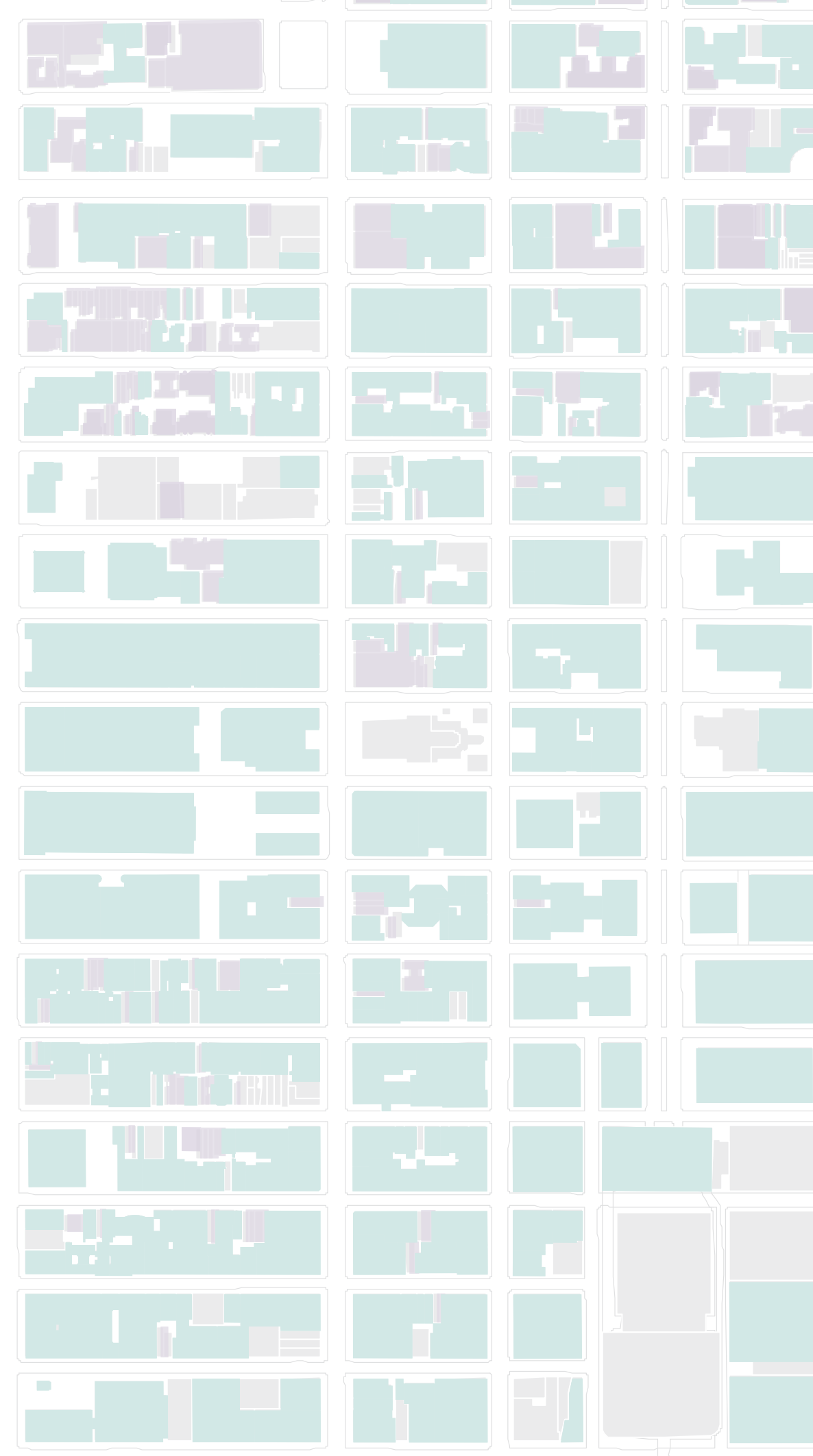

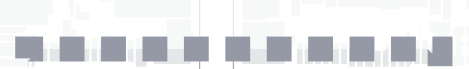

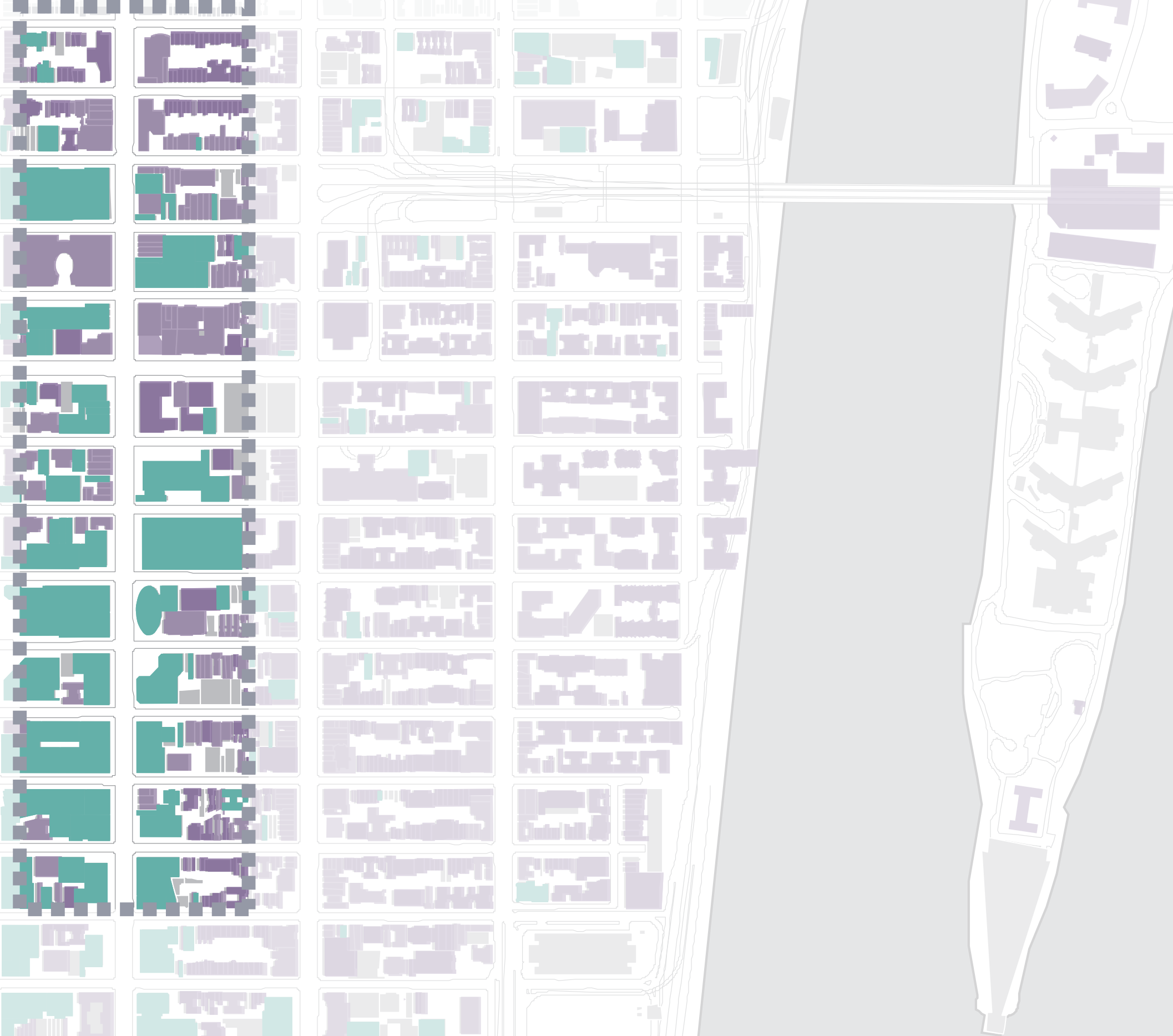




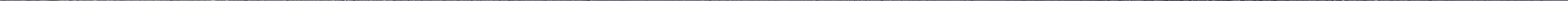


The
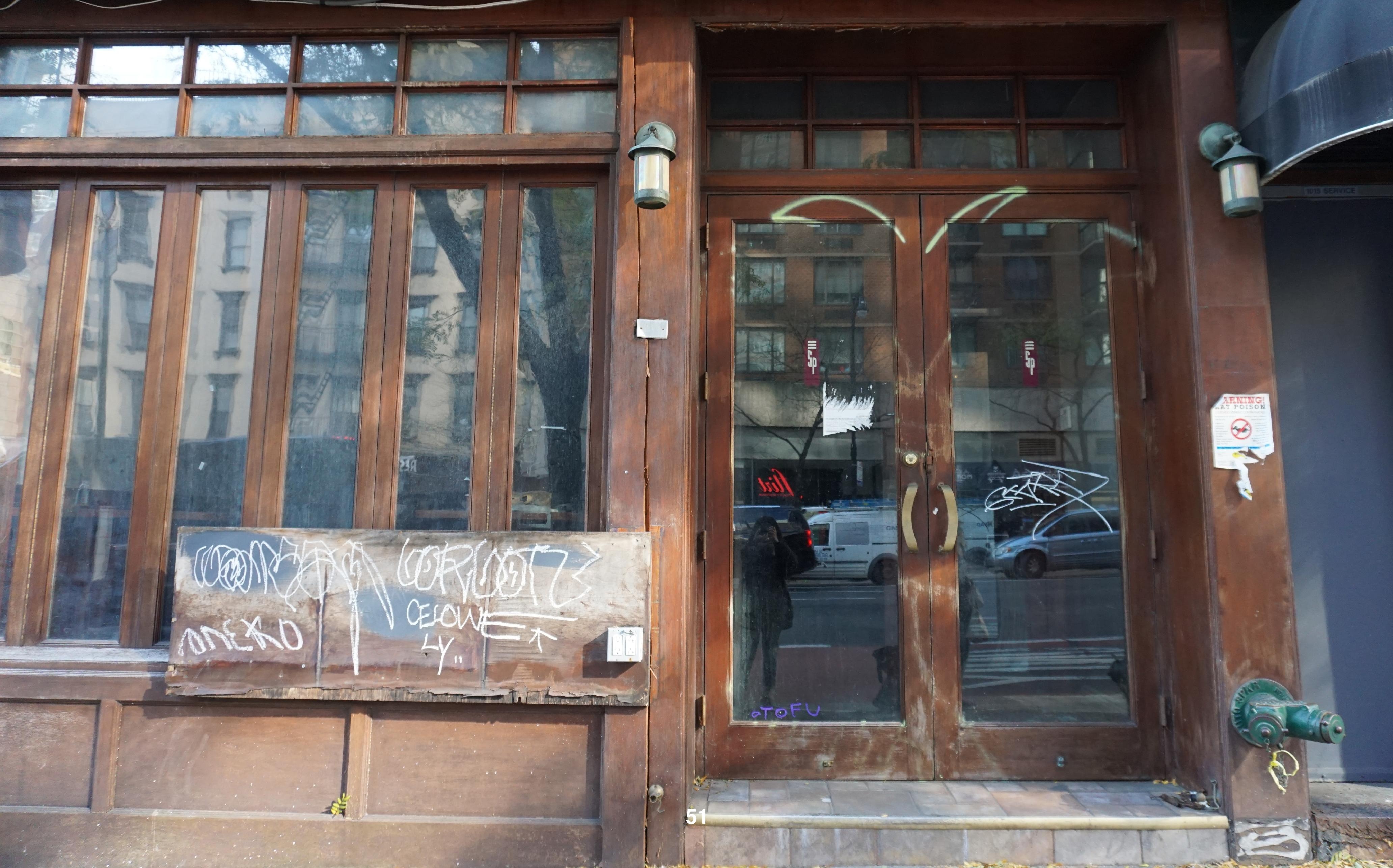


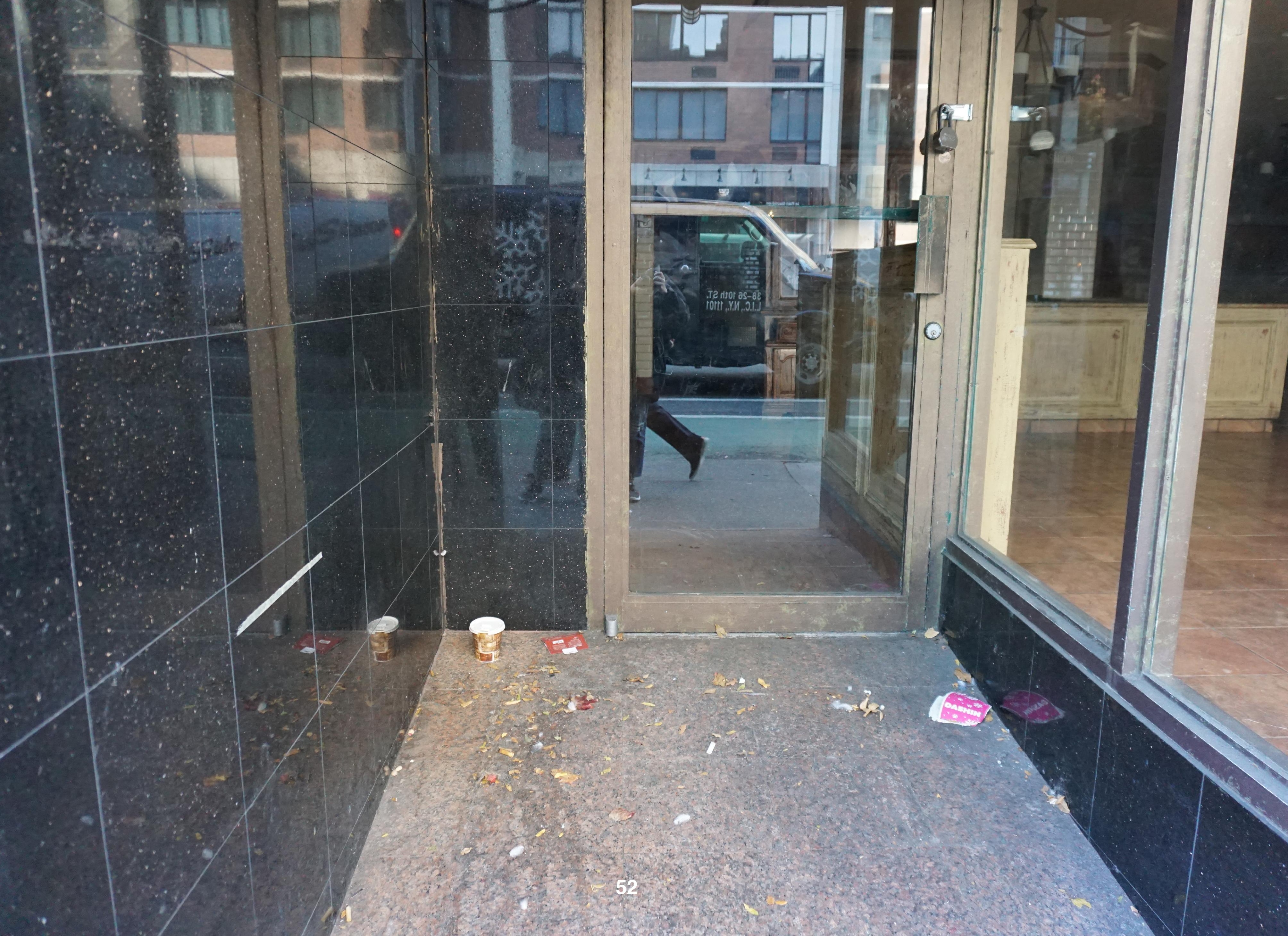




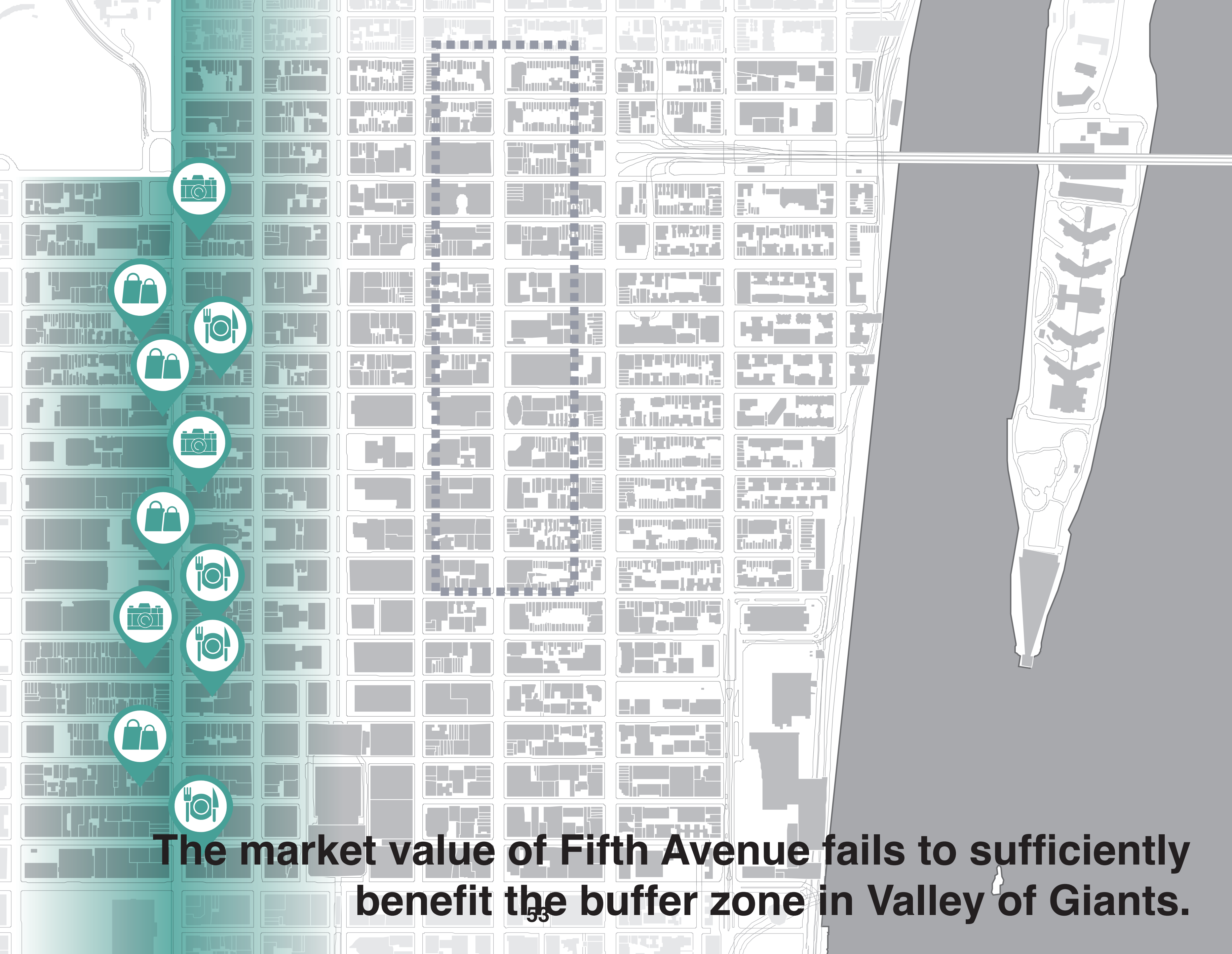




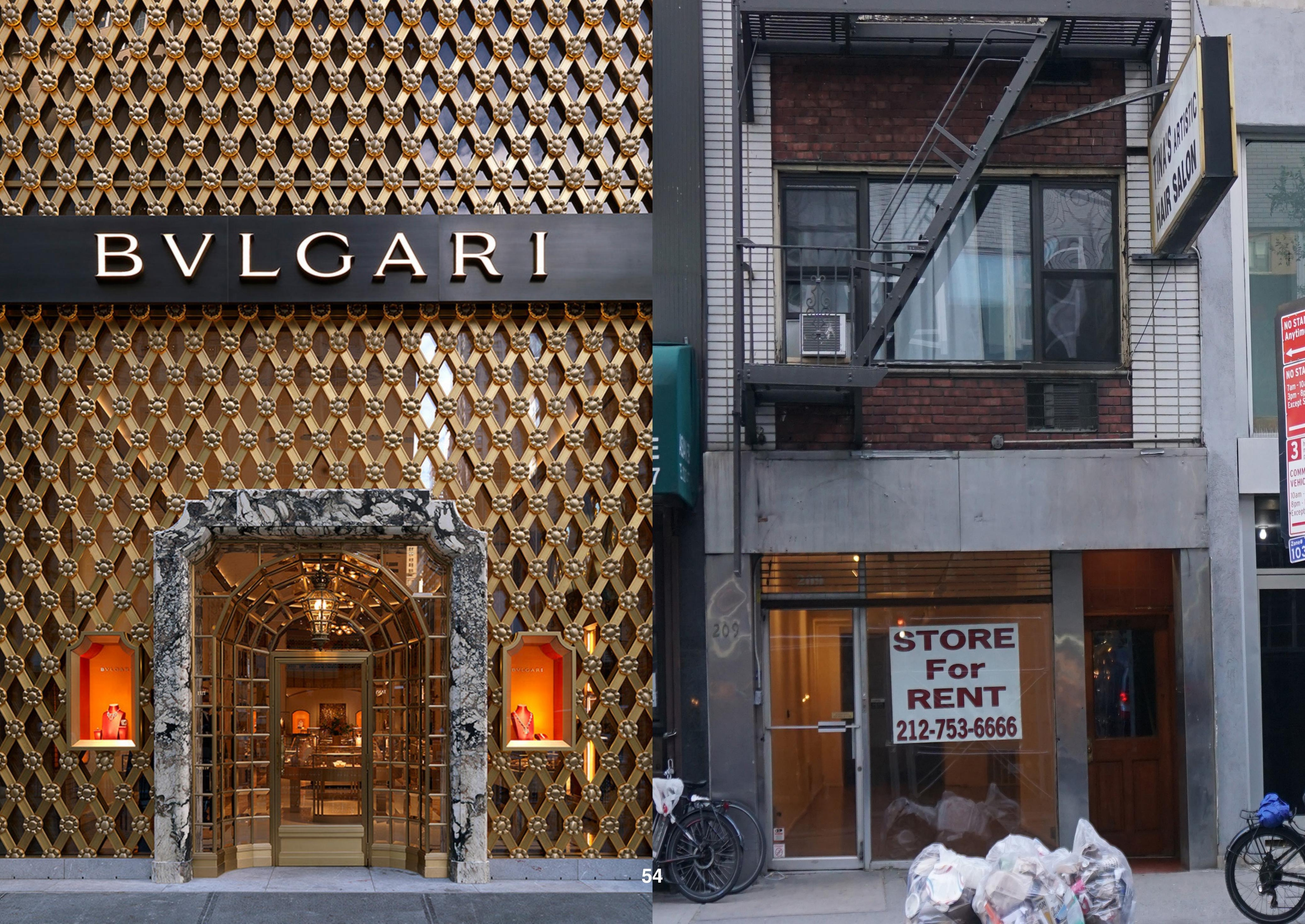




\section{PROBLEM}

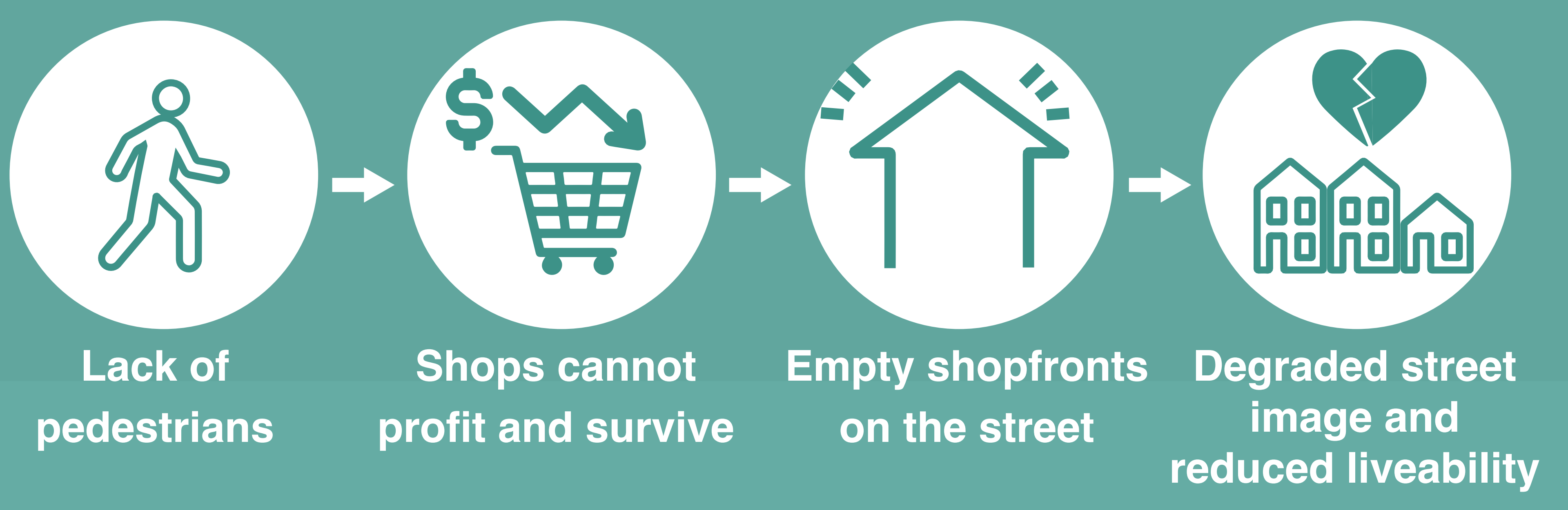




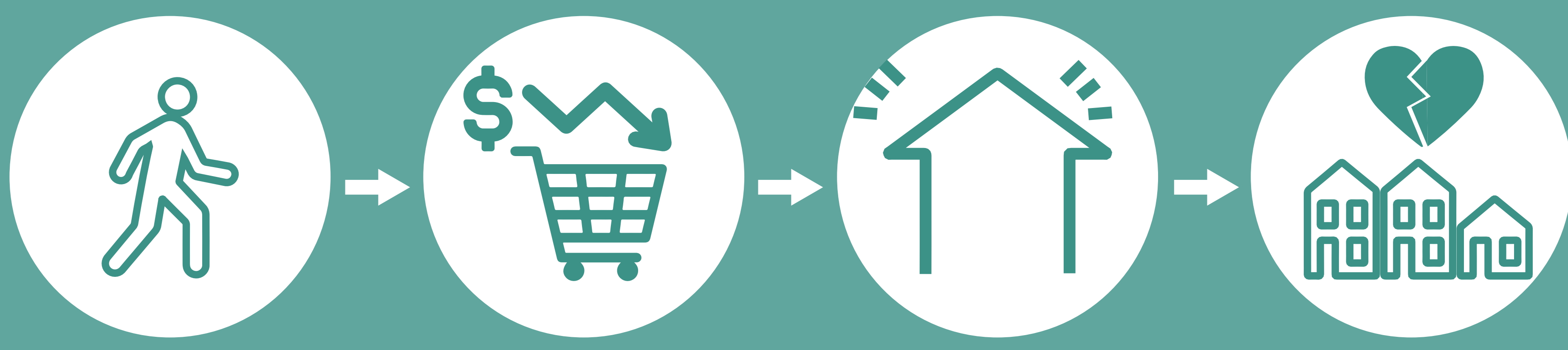

\section{SOLUTION}

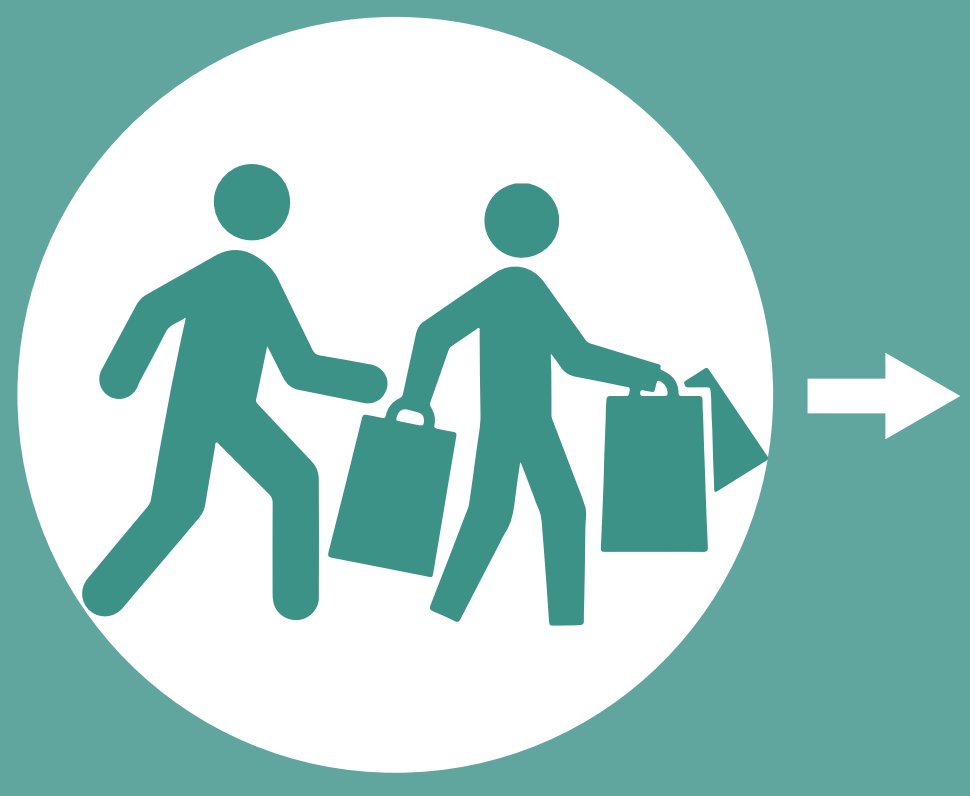

More pedestiran flow

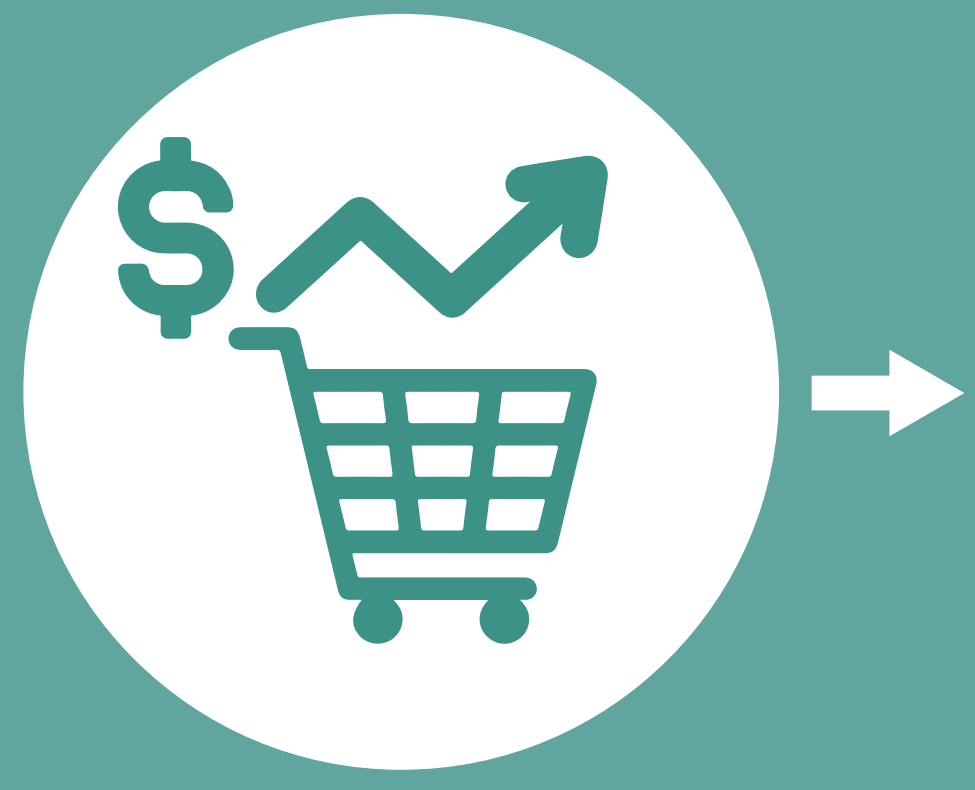

Shops become more profitable
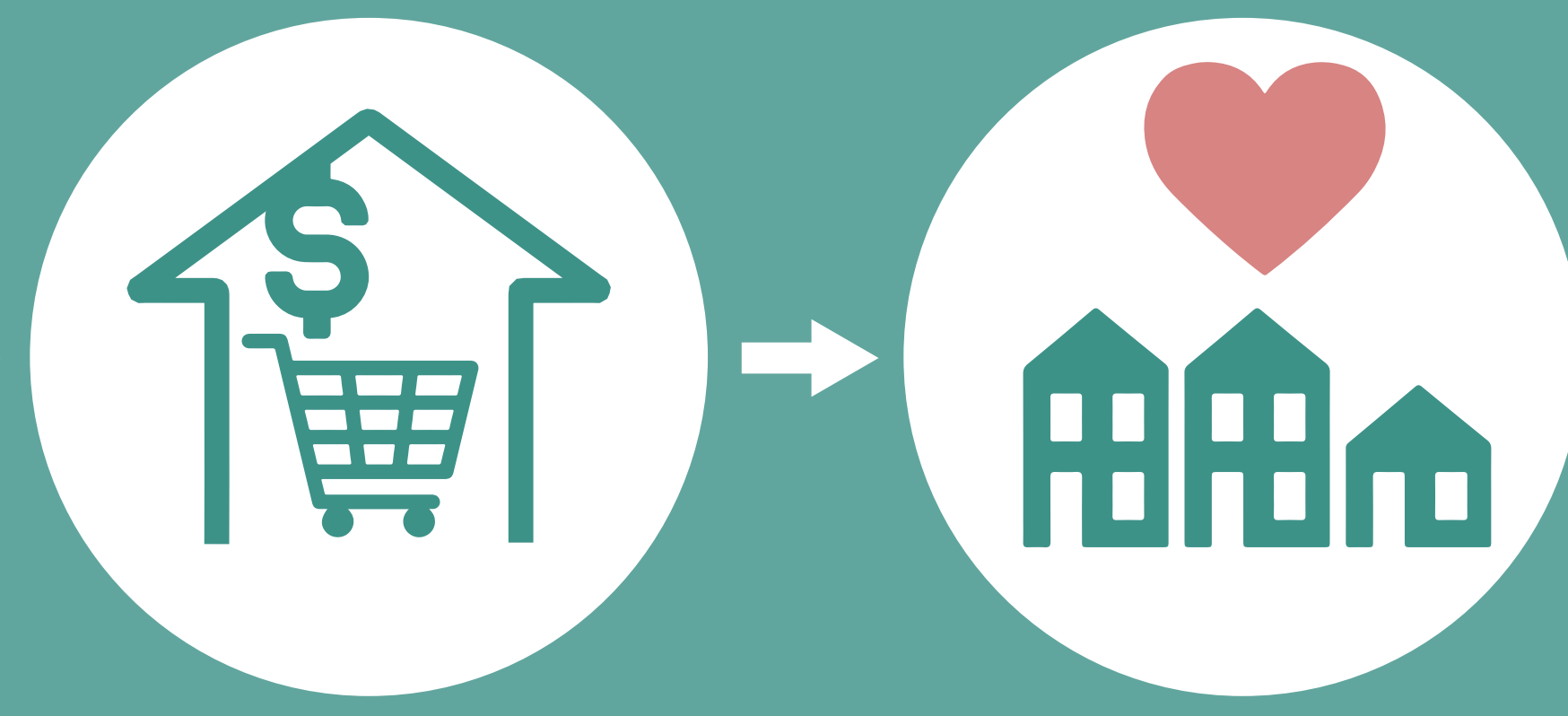

More retails move Higher liveability into the vacancies of the city 


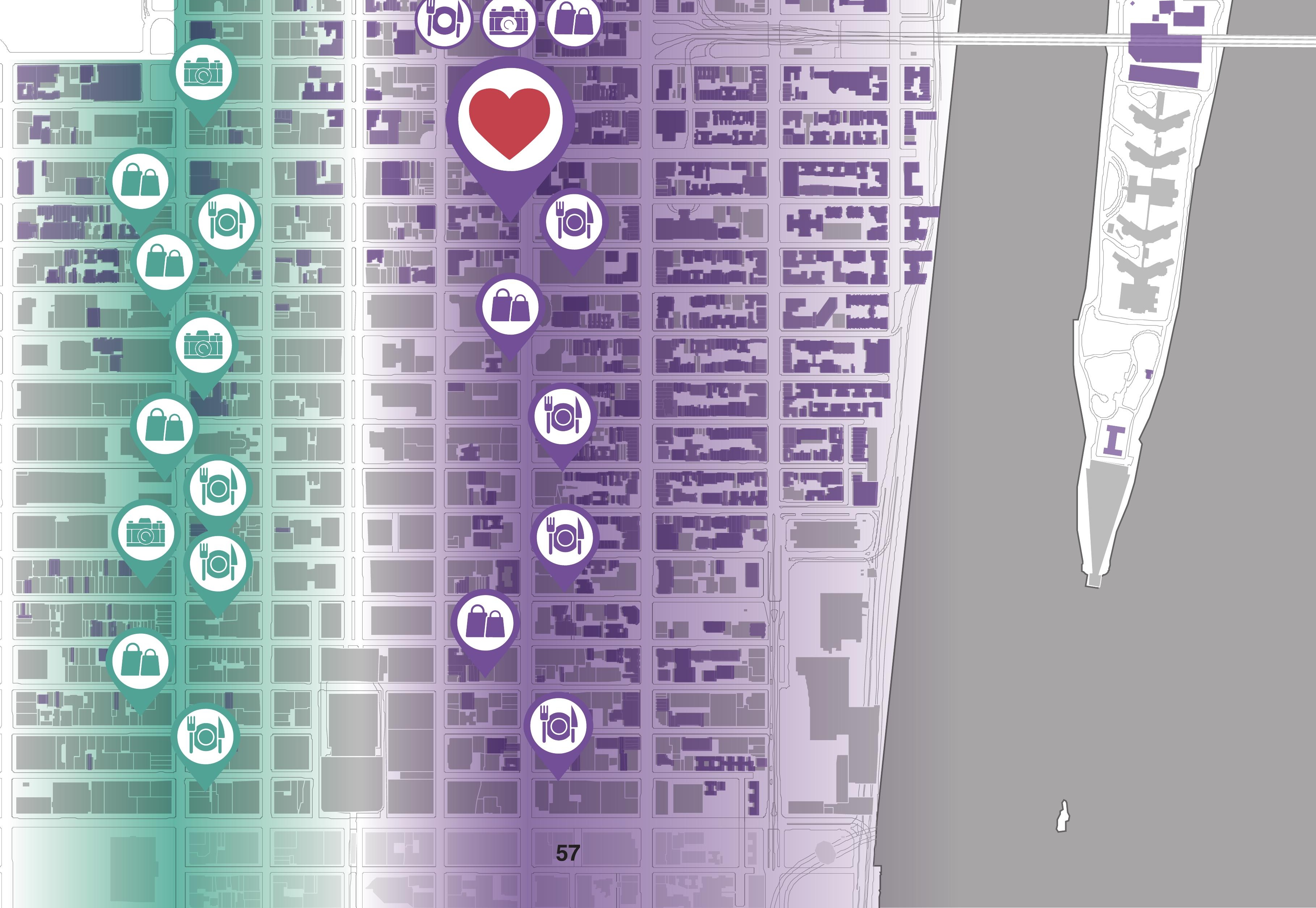




\section{Brief}




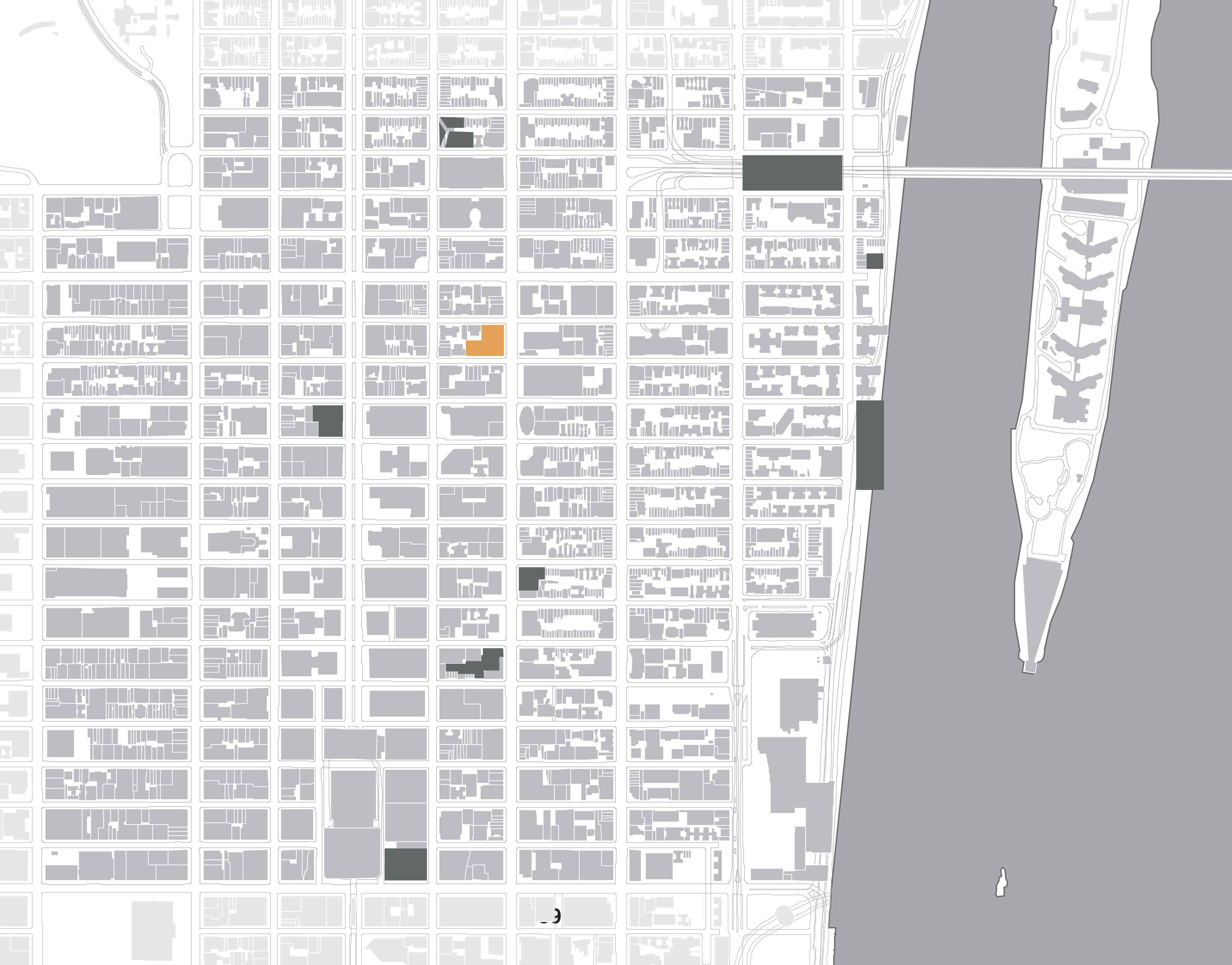




\section{Location}
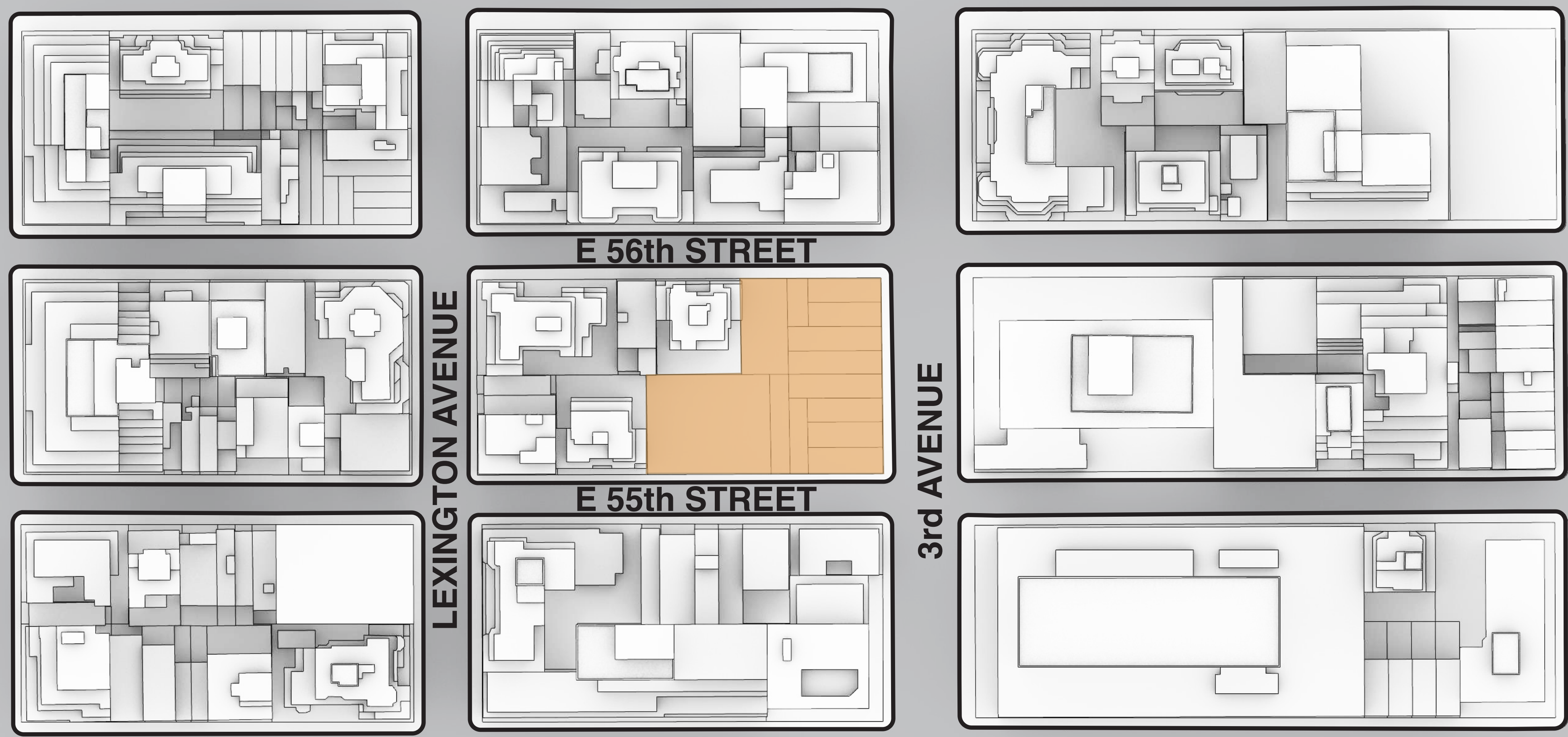


\section{Functions}
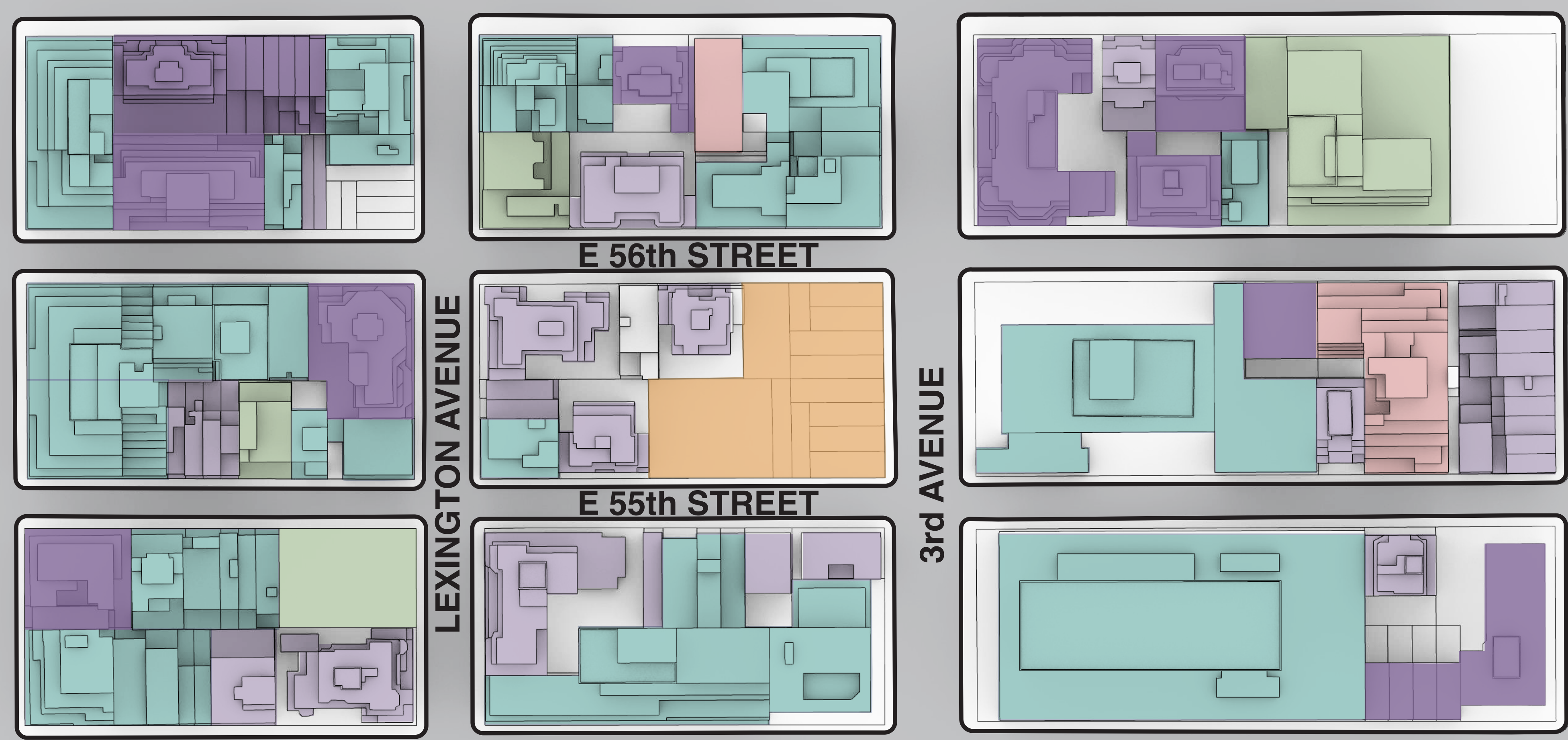


\section{Solar Radiation}
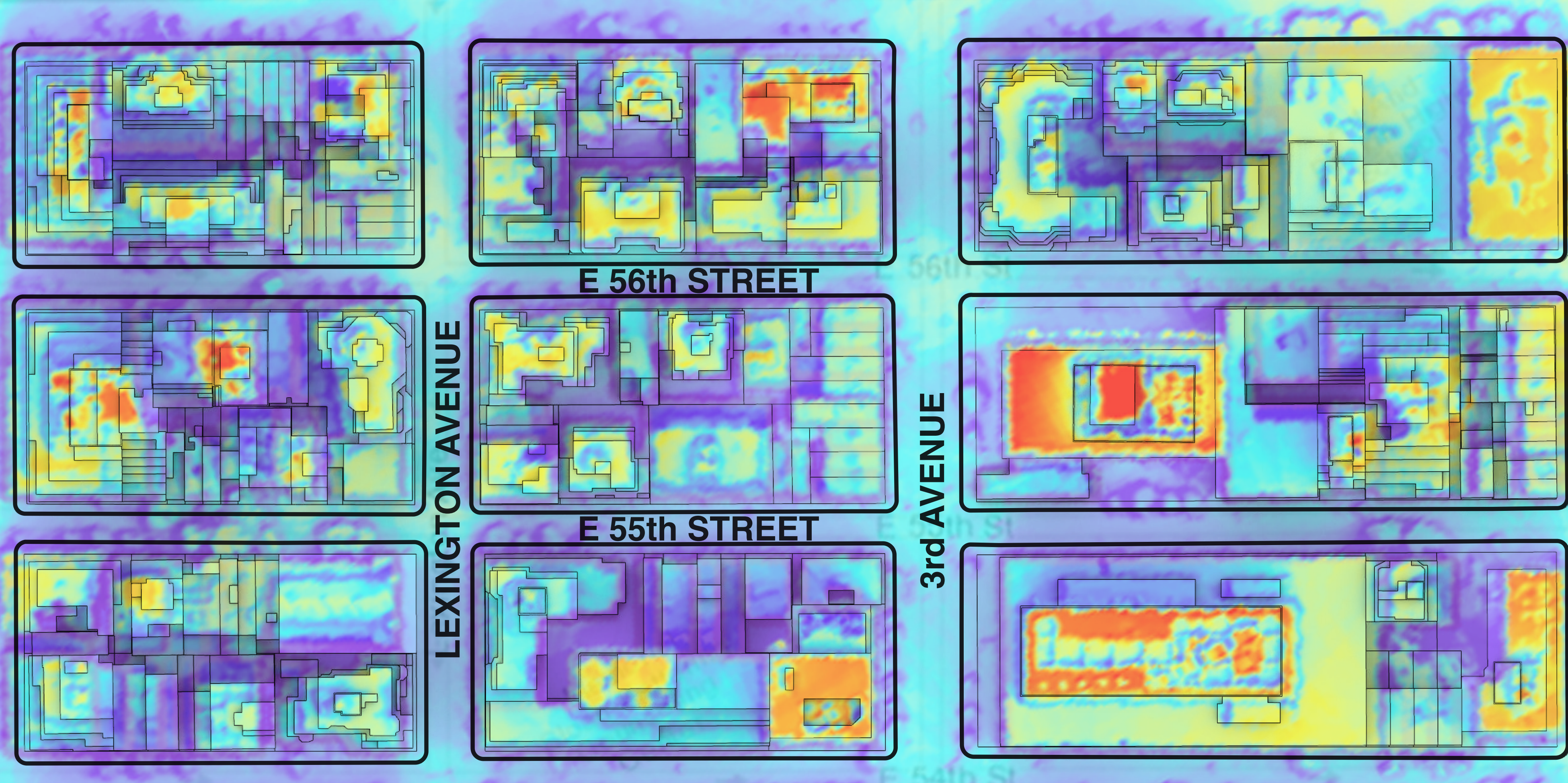


\section{Current Situation}

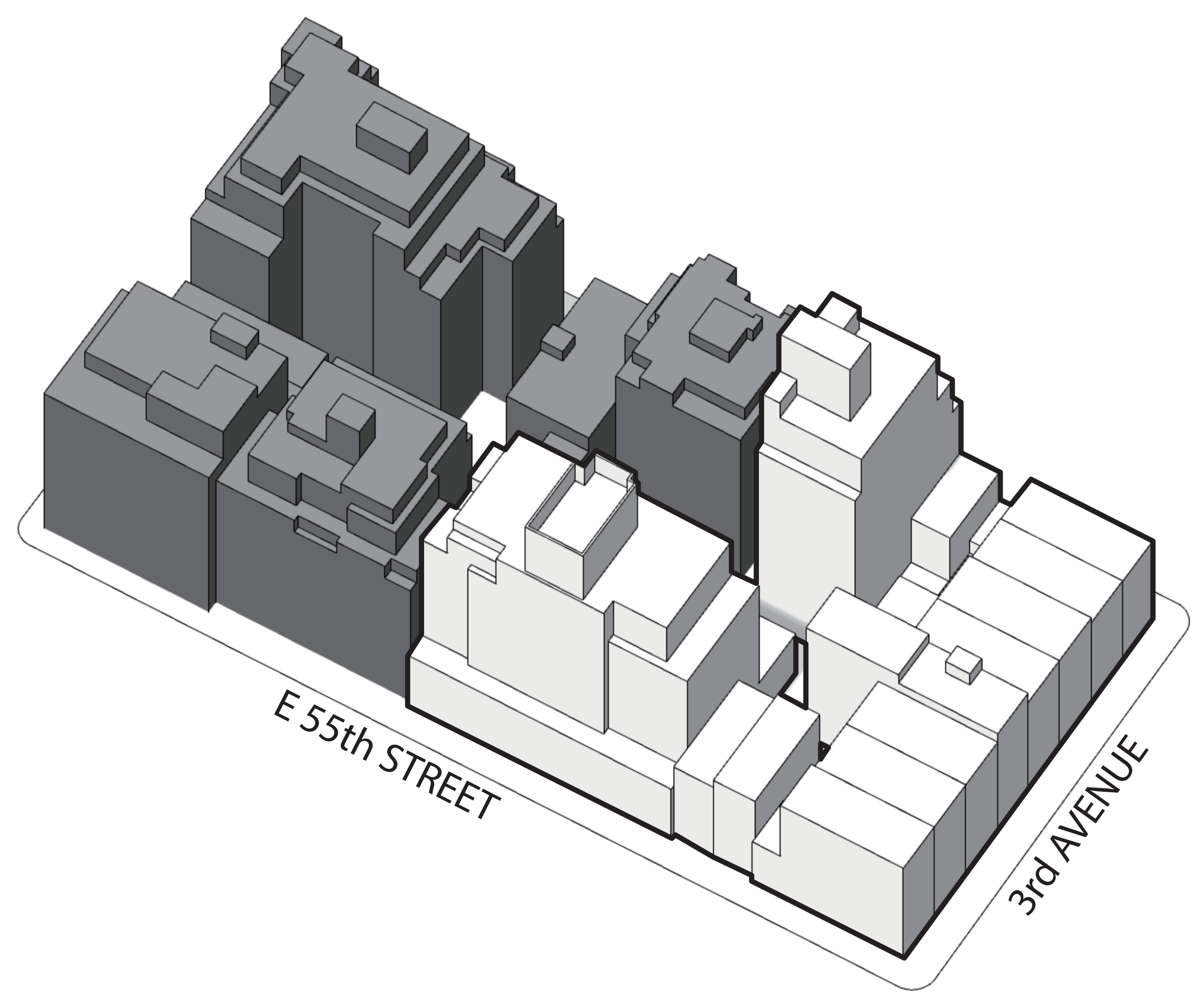




\section{Current Situation}

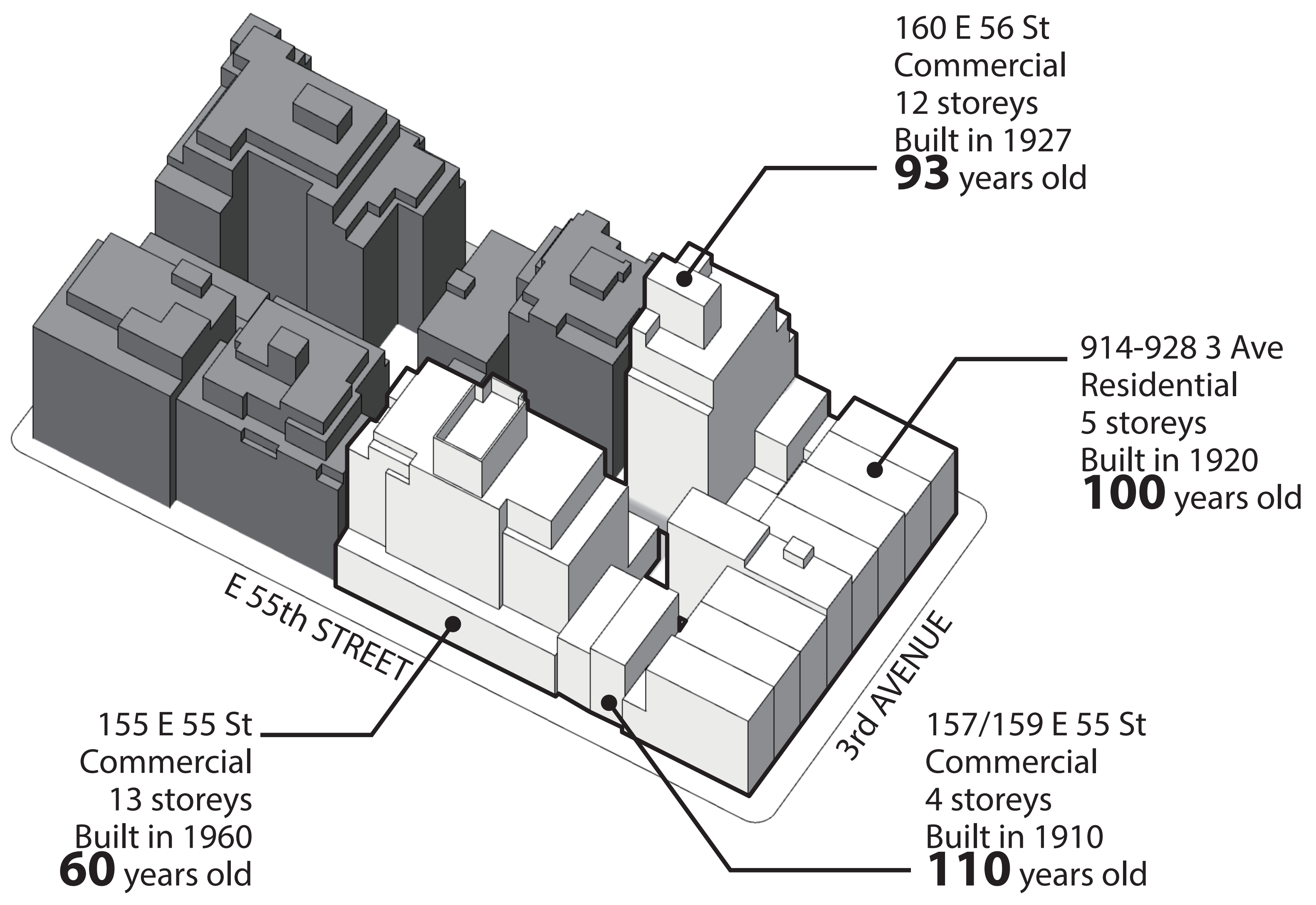




\section{Building Lot}

Lot: $3,800 \mathrm{~m}^{2}$

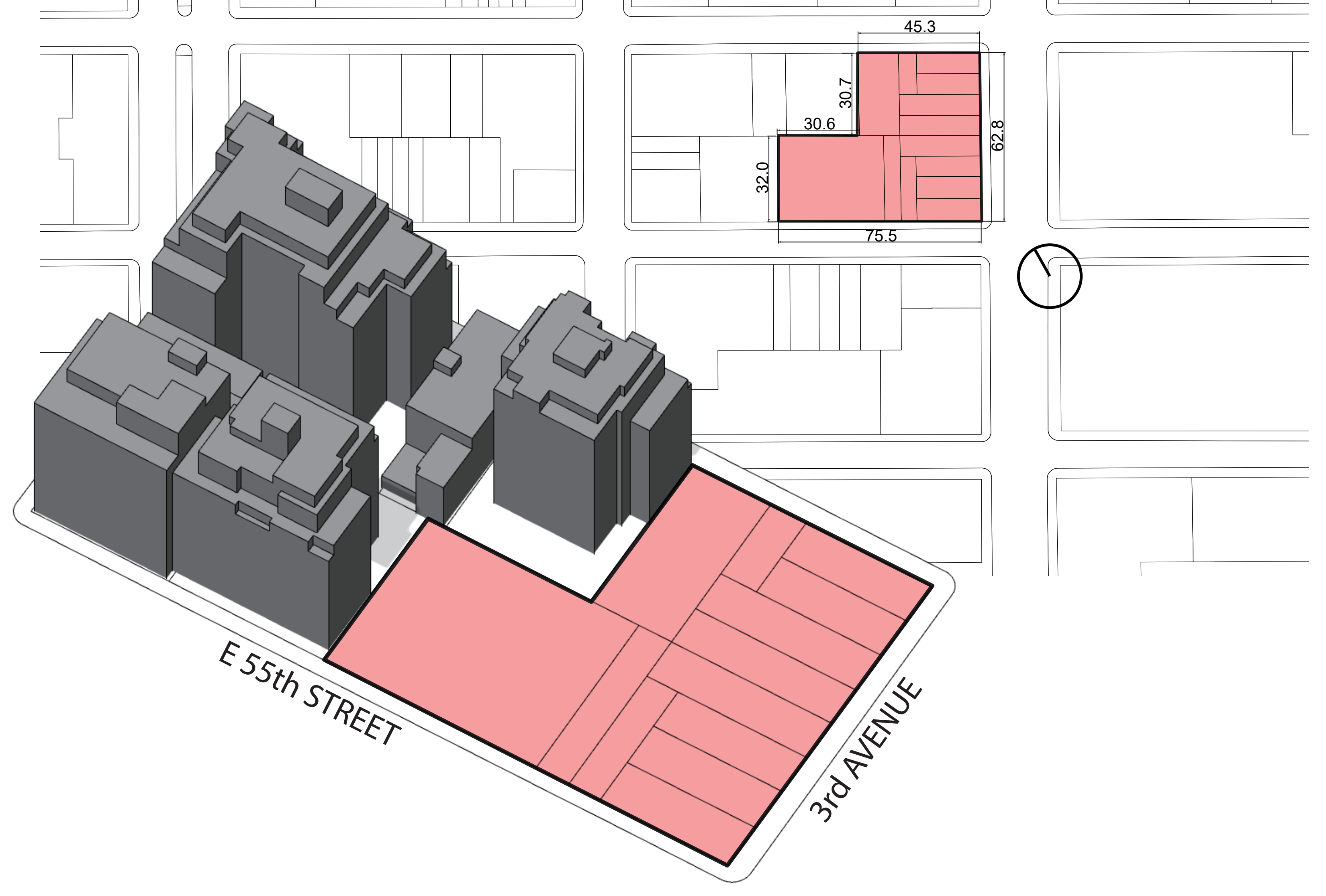




\section{Maximum Volume}

C6-6 Corporate headquarters, large hotels, department stores and entertainment facilities in high-rise mixed builldings are permitted

Lot: $3,800 \mathrm{~m}^{2}$

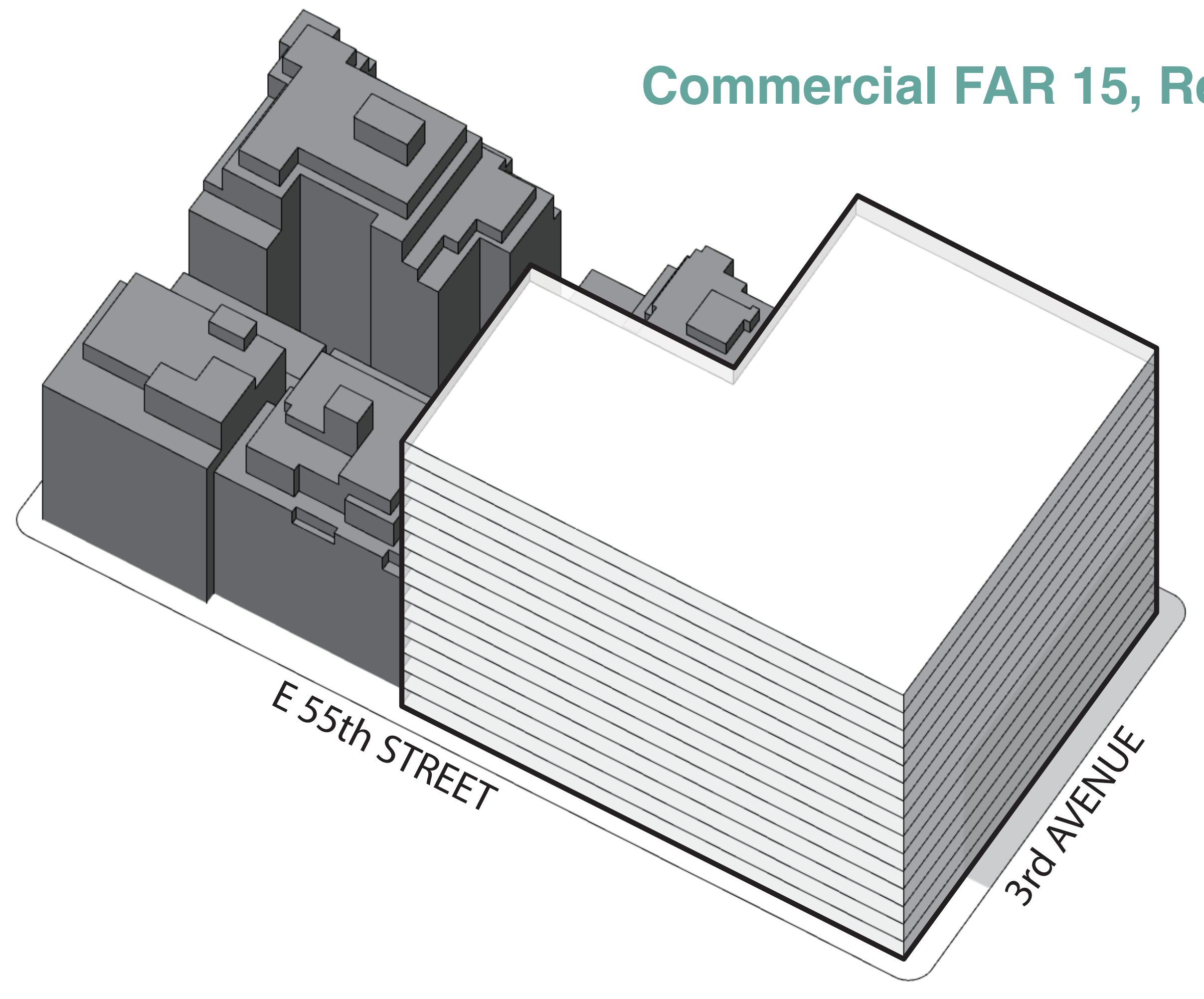

Height: $60 \mathrm{~m}$

GFA: $57,000 \mathrm{~m}^{2}$

FAR: 15 


\section{Main Programmes}

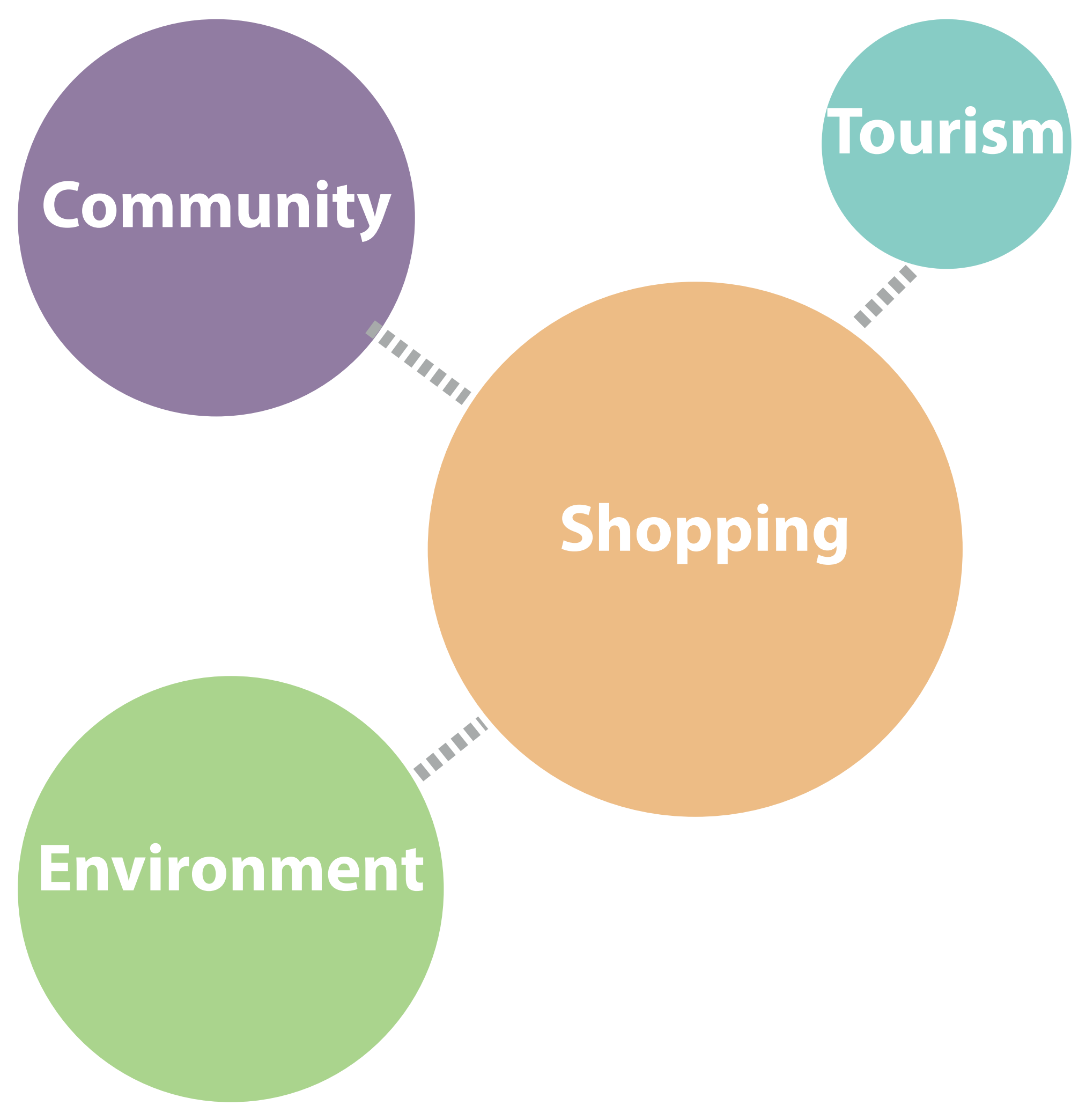




\section{Main Programmes}

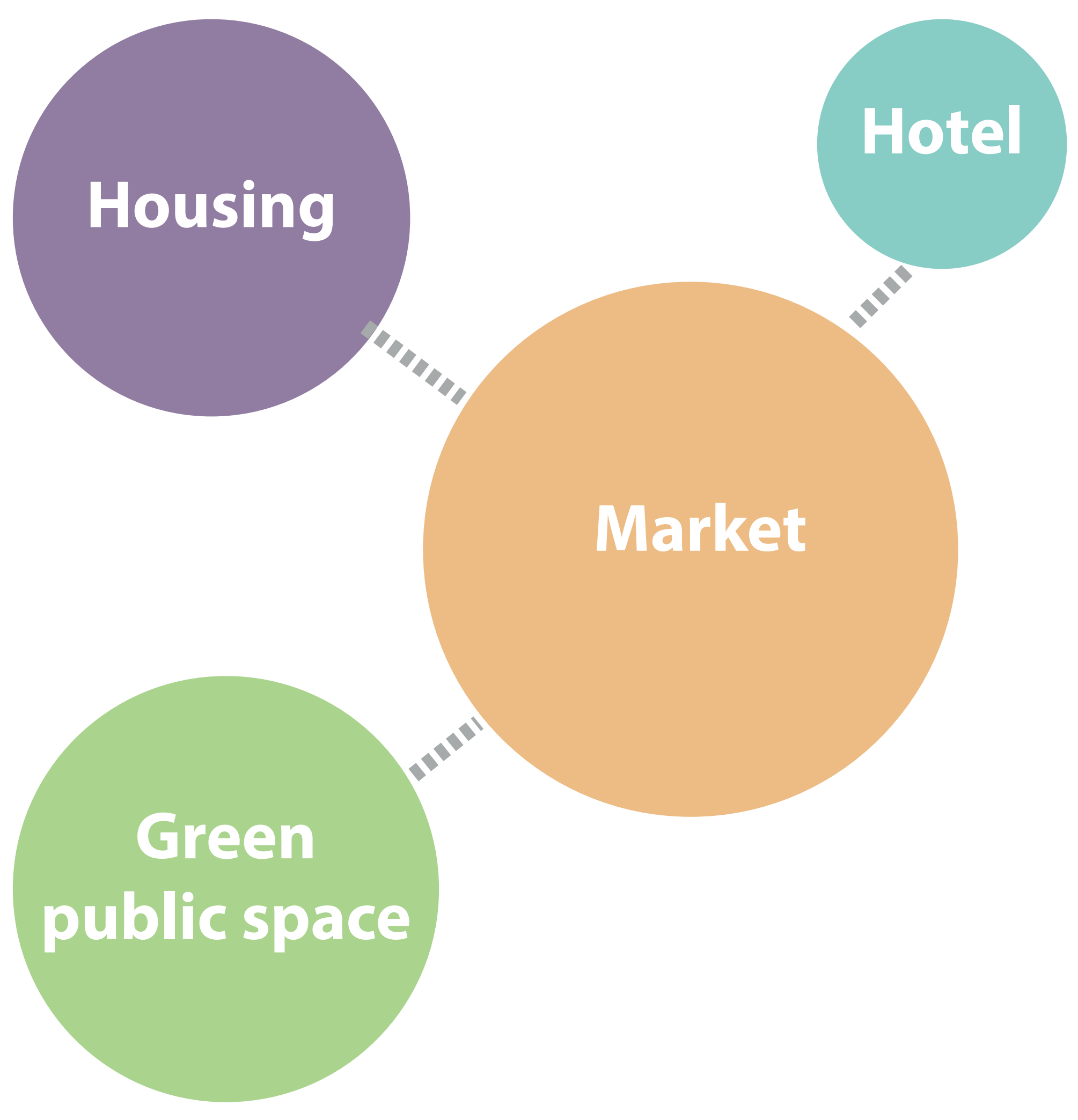




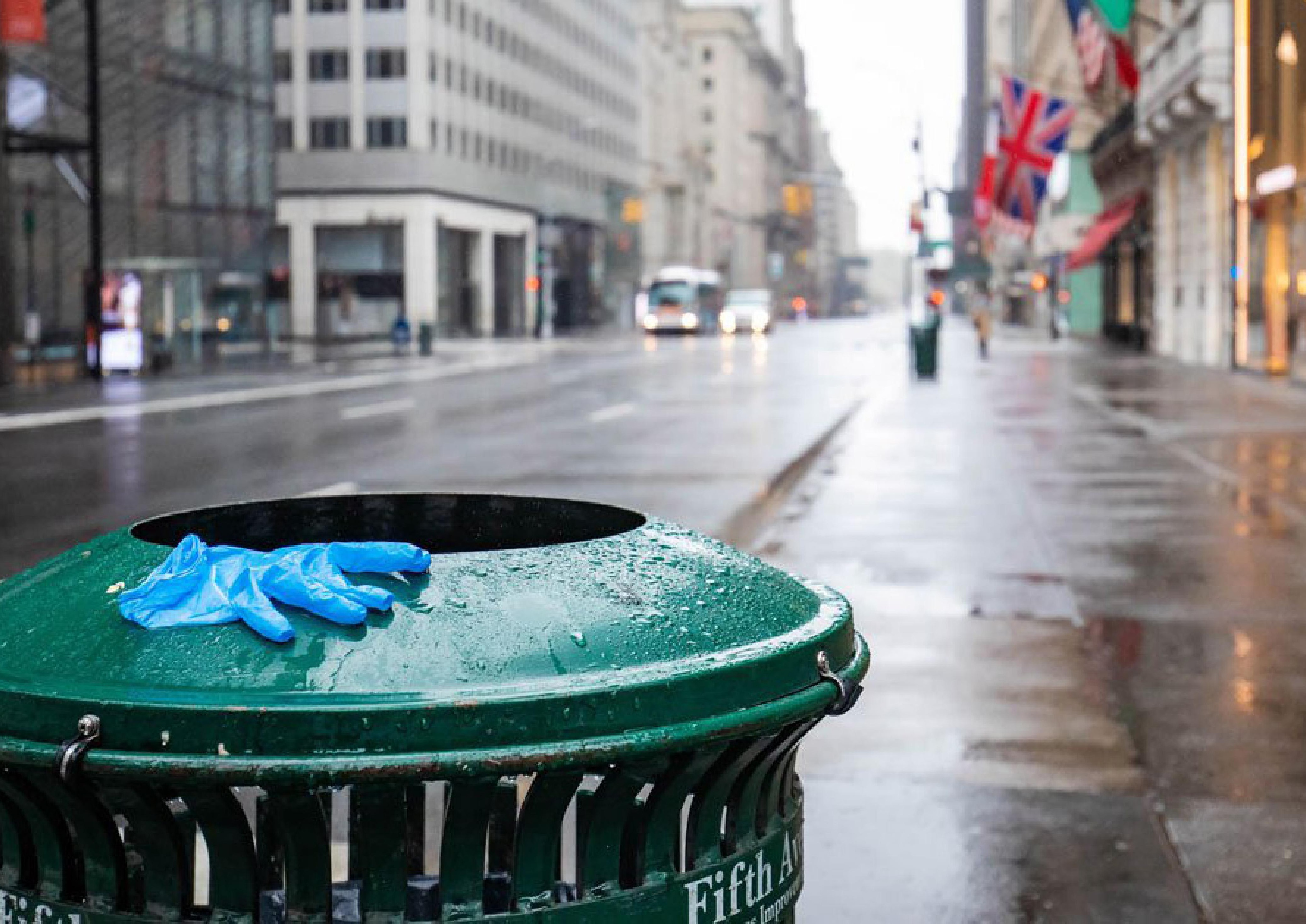




\section{Main Programmes}

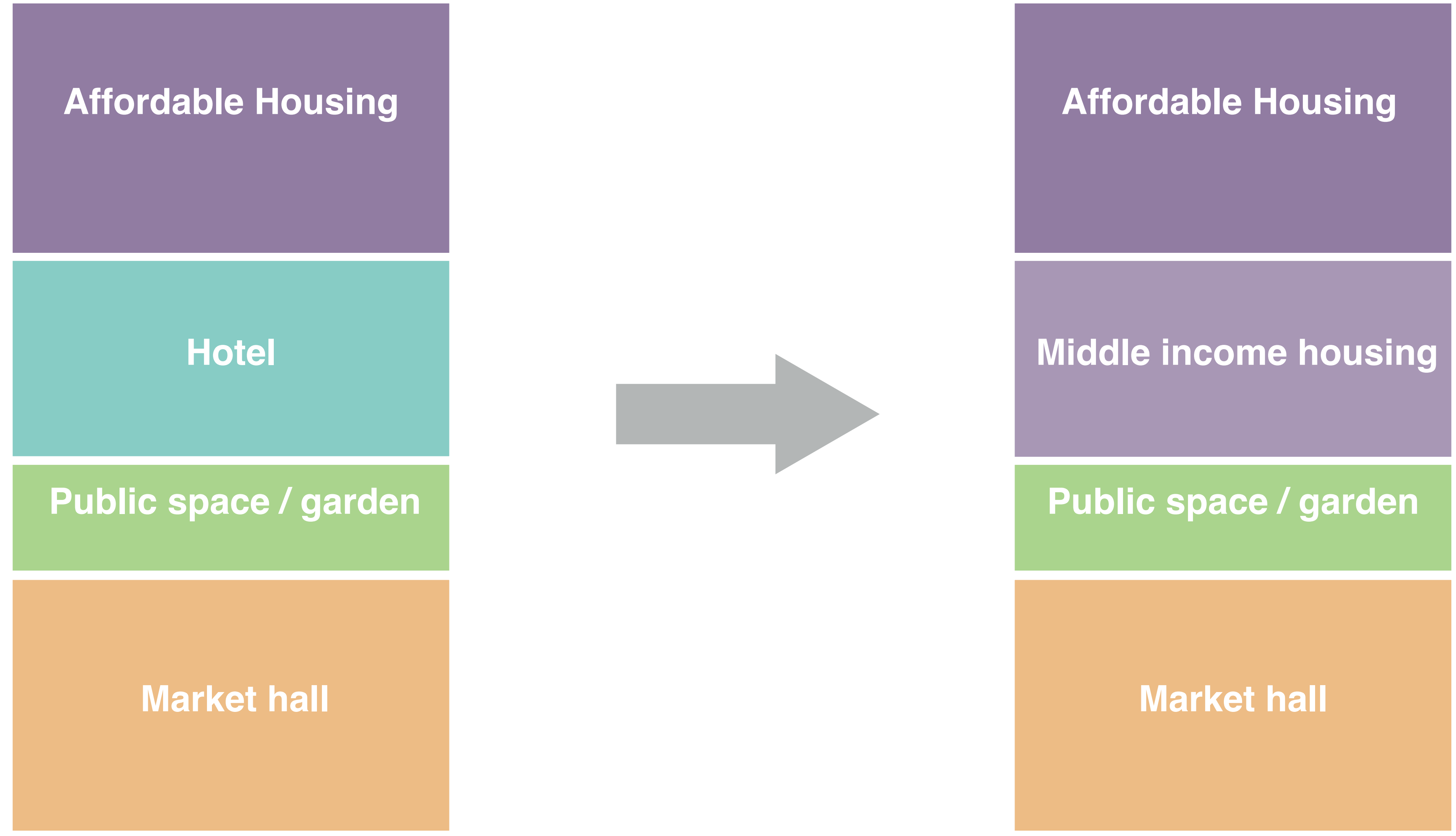




\section{Main Programmes}

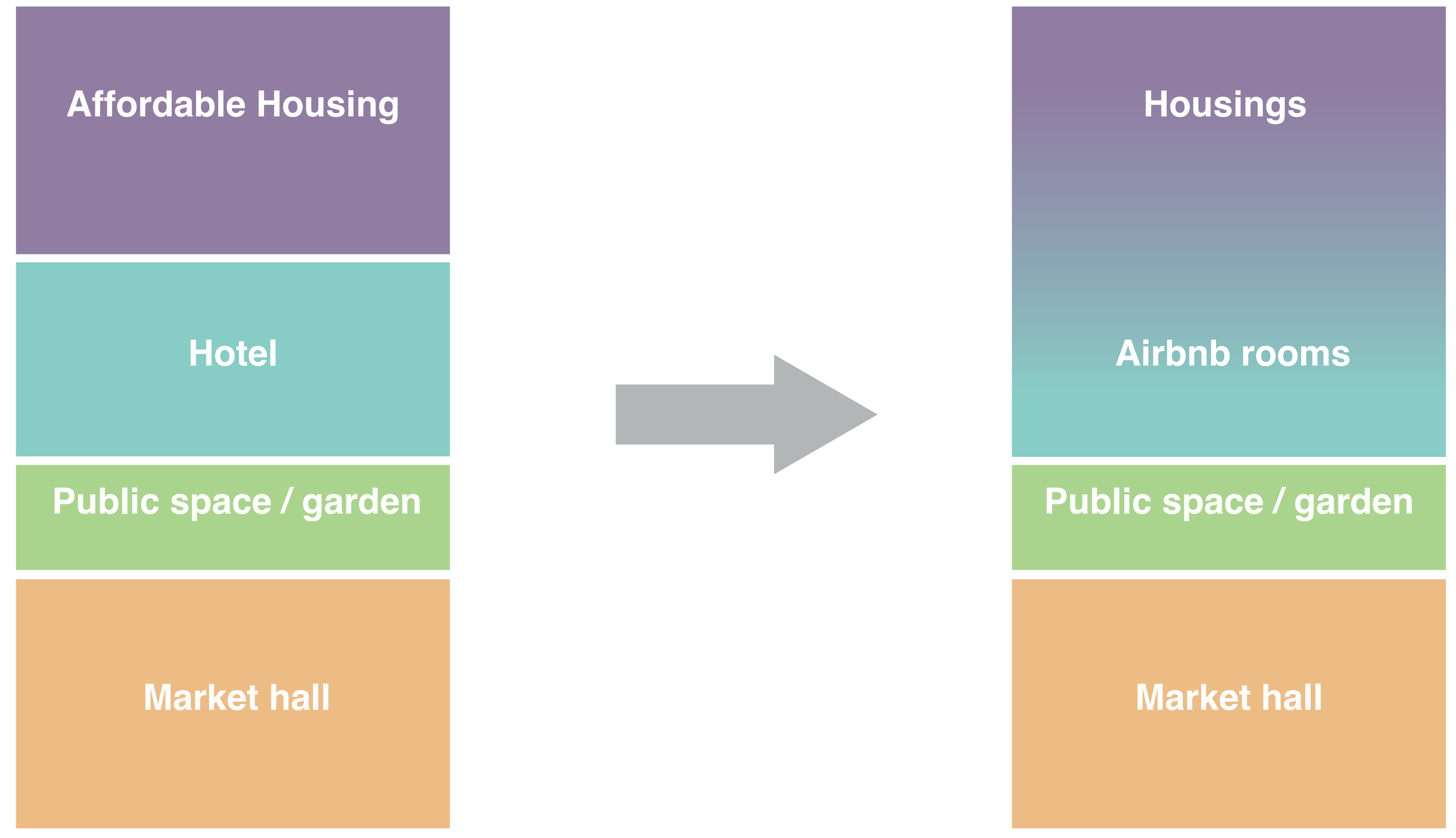



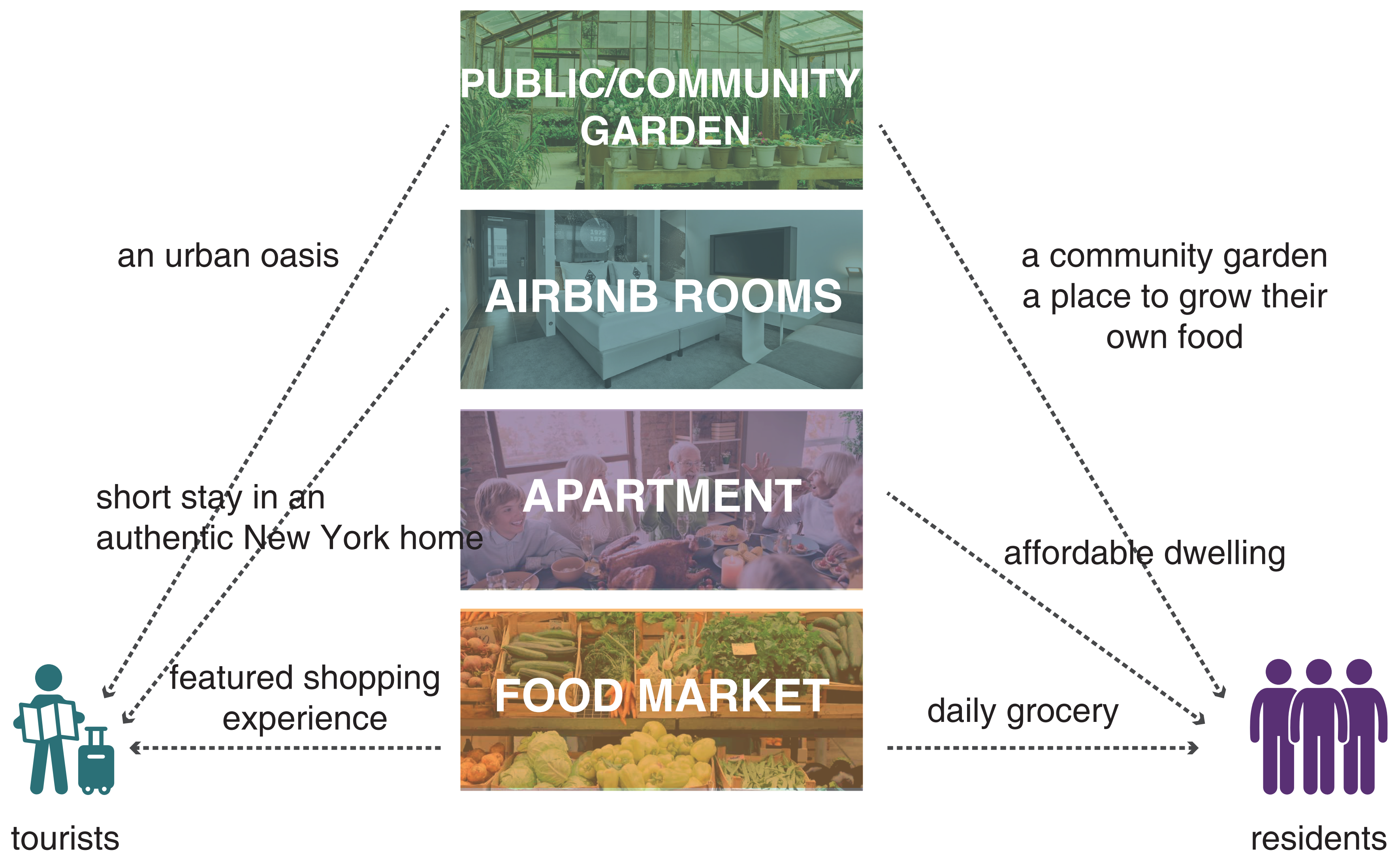

tourists 


\section{Programme ratio}

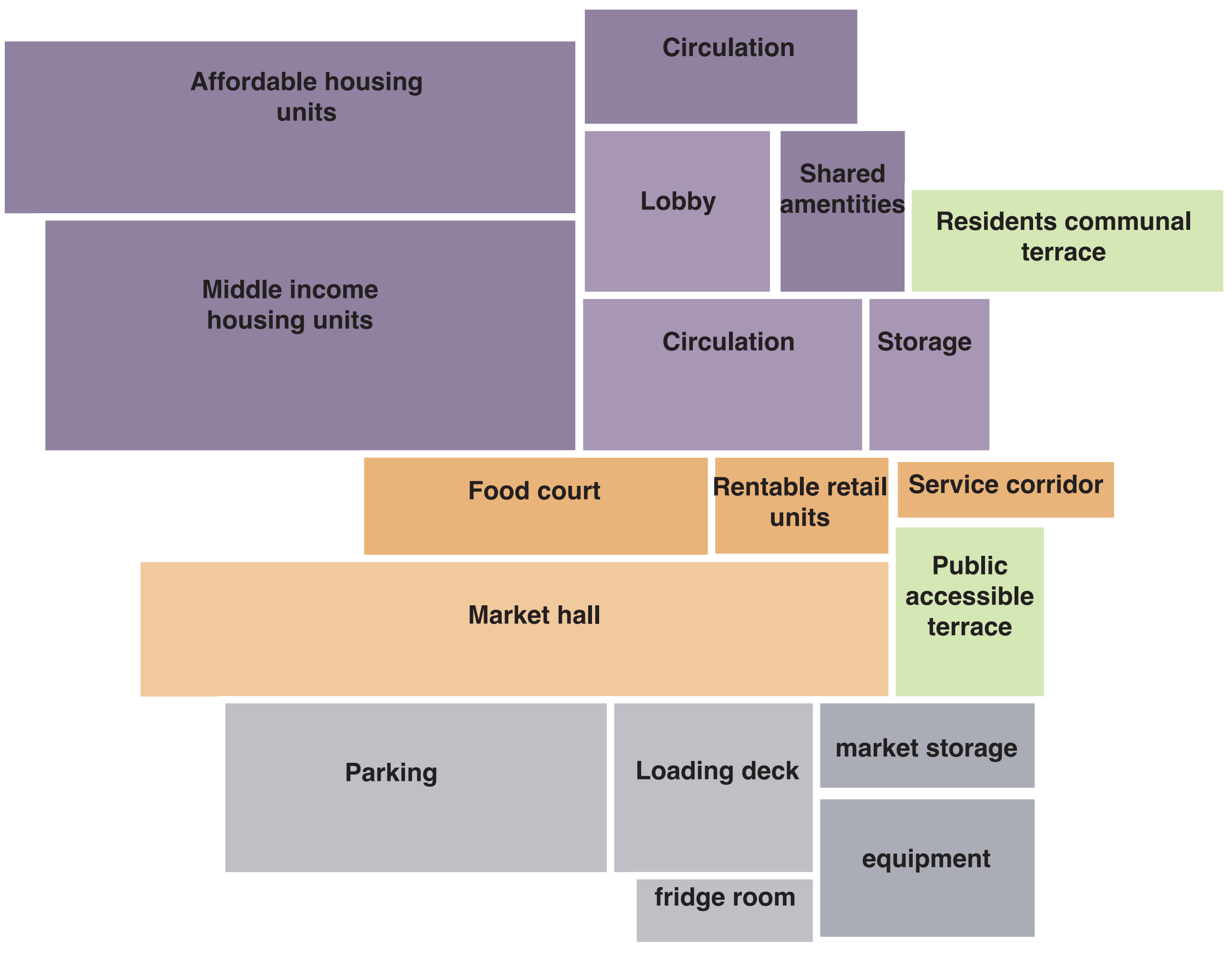


Ambitions

\section{A FOOD MARKET aims to promote an alternative LIFESTYLE against ever-expanding unsustainable} consumerism in our society by making grocery shopping AN ATTRACTIVE AND ENJOYABLE EXPERIENCE that can partially replace recreational shopping. 
Design Concept 


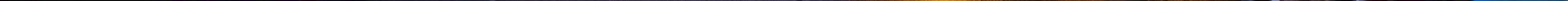




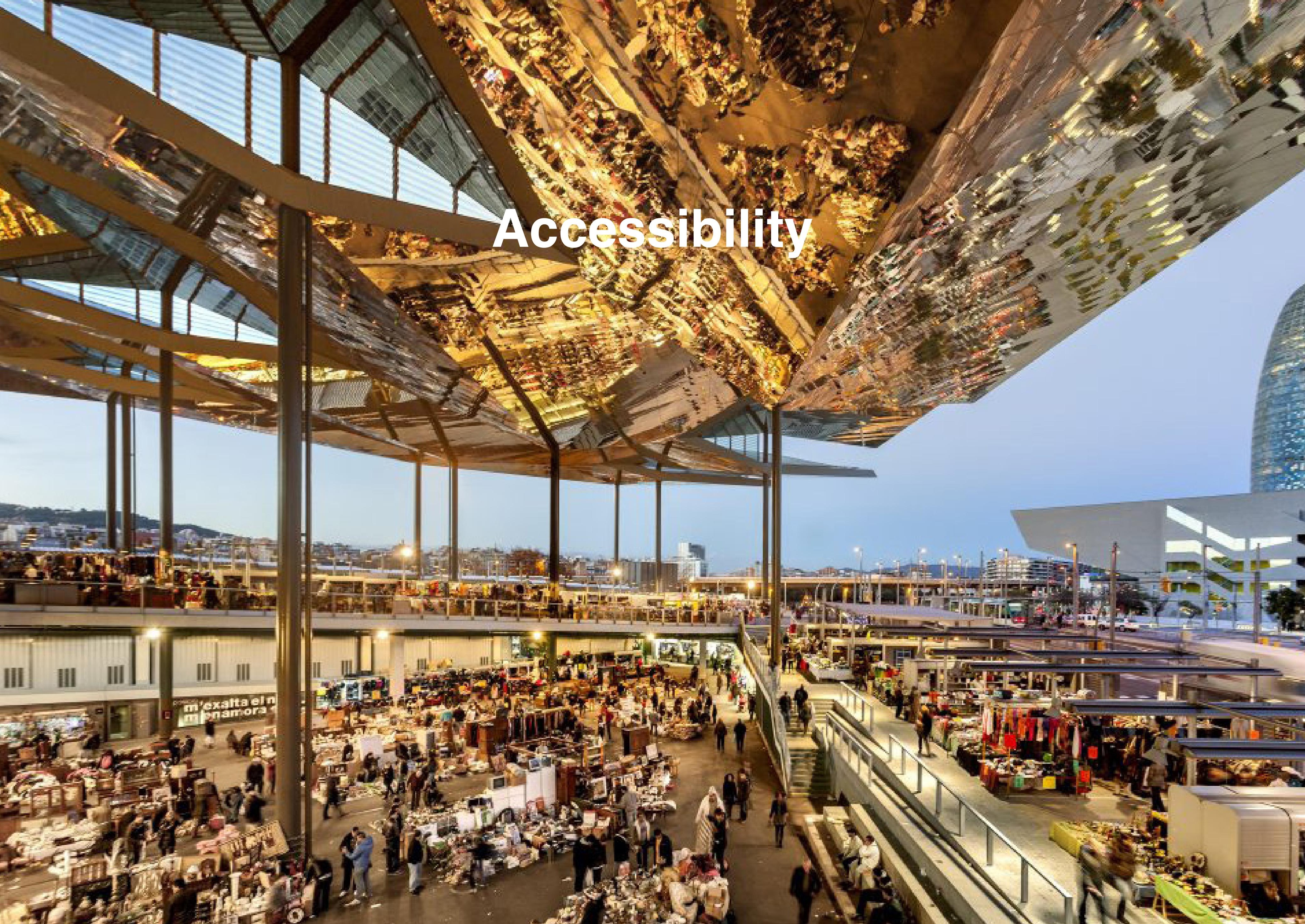




\section{Spatial concepts}

Publicity

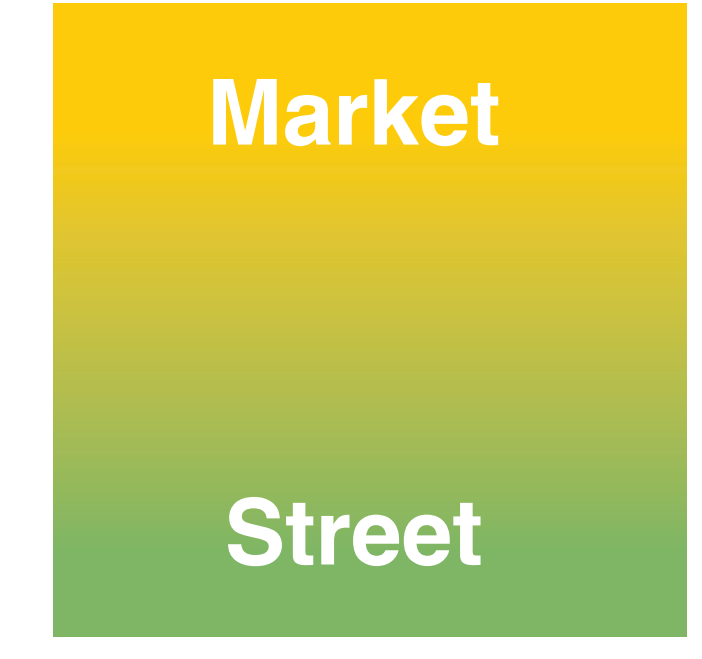

Blurred boundary between the market and urban street
Verticality

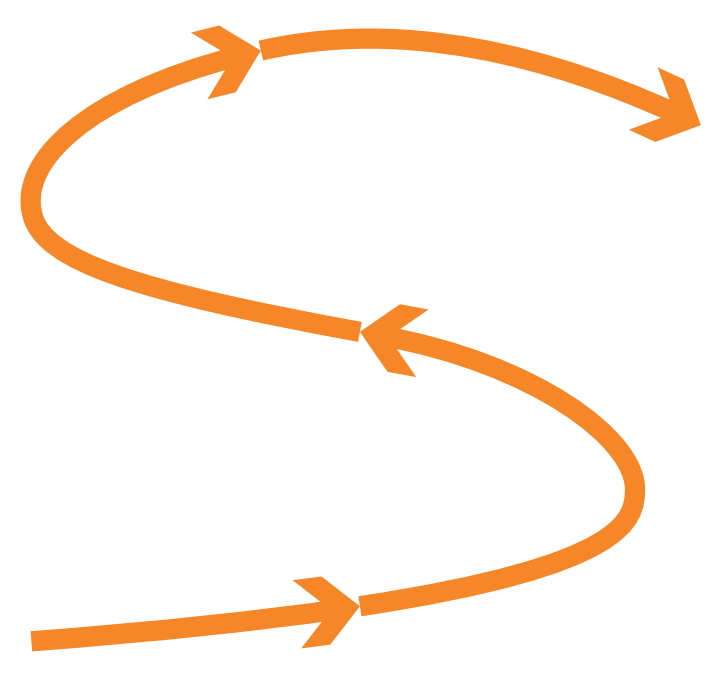

A continuous walk to take...
Recreation

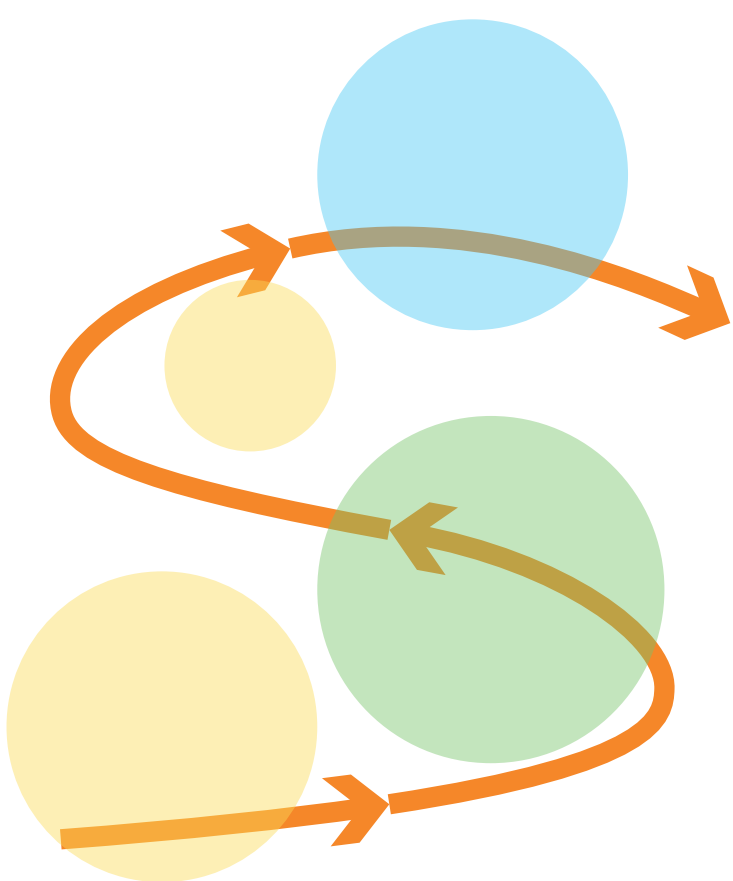

...alongside with diverse and enjoyable shopping experience
Accessiblity

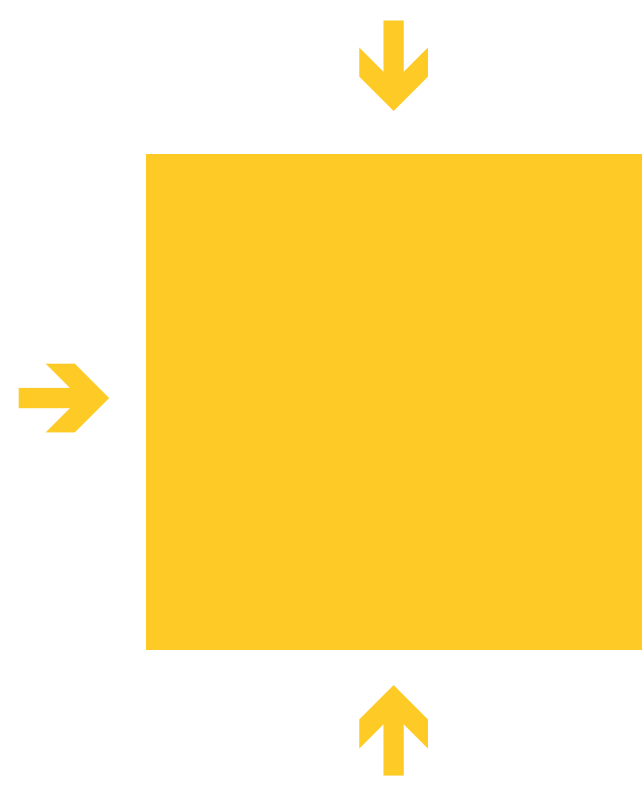

Market as big public space with easy access 

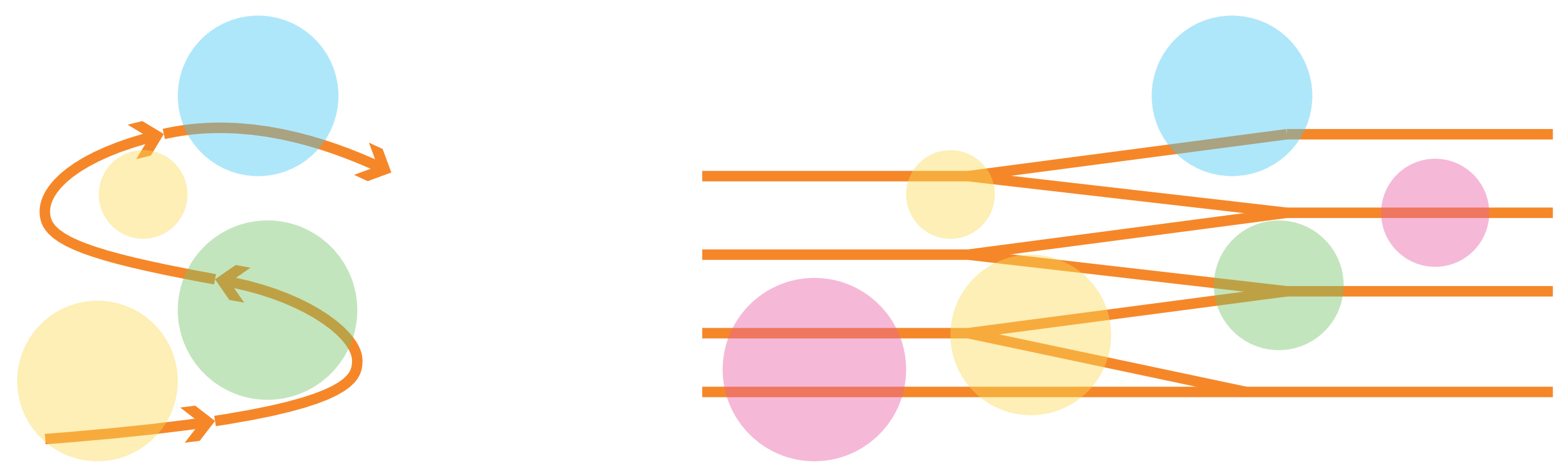

Staggered floors connected by ramps 


\section{Plot}

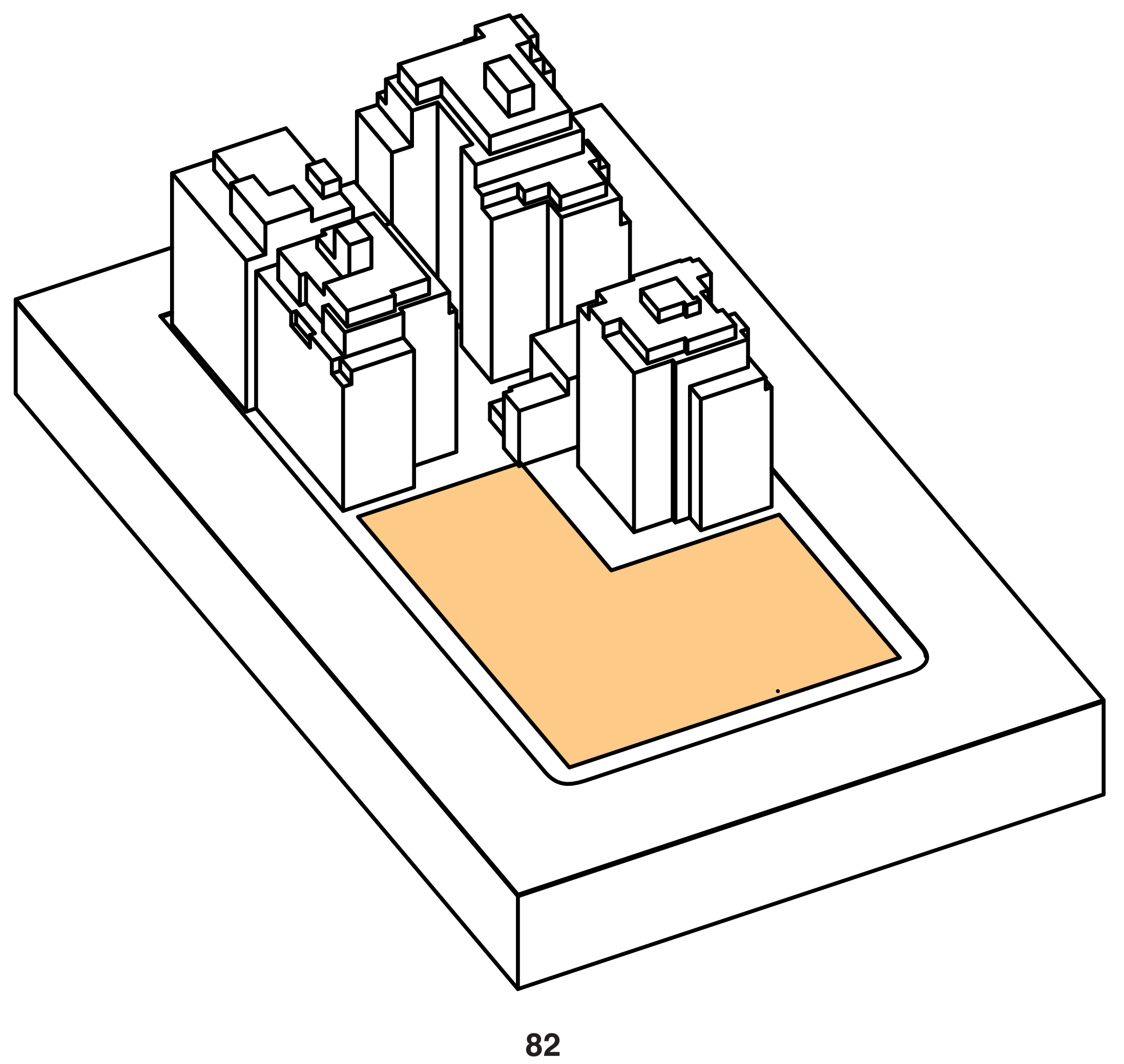




\section{Podium Volumn}

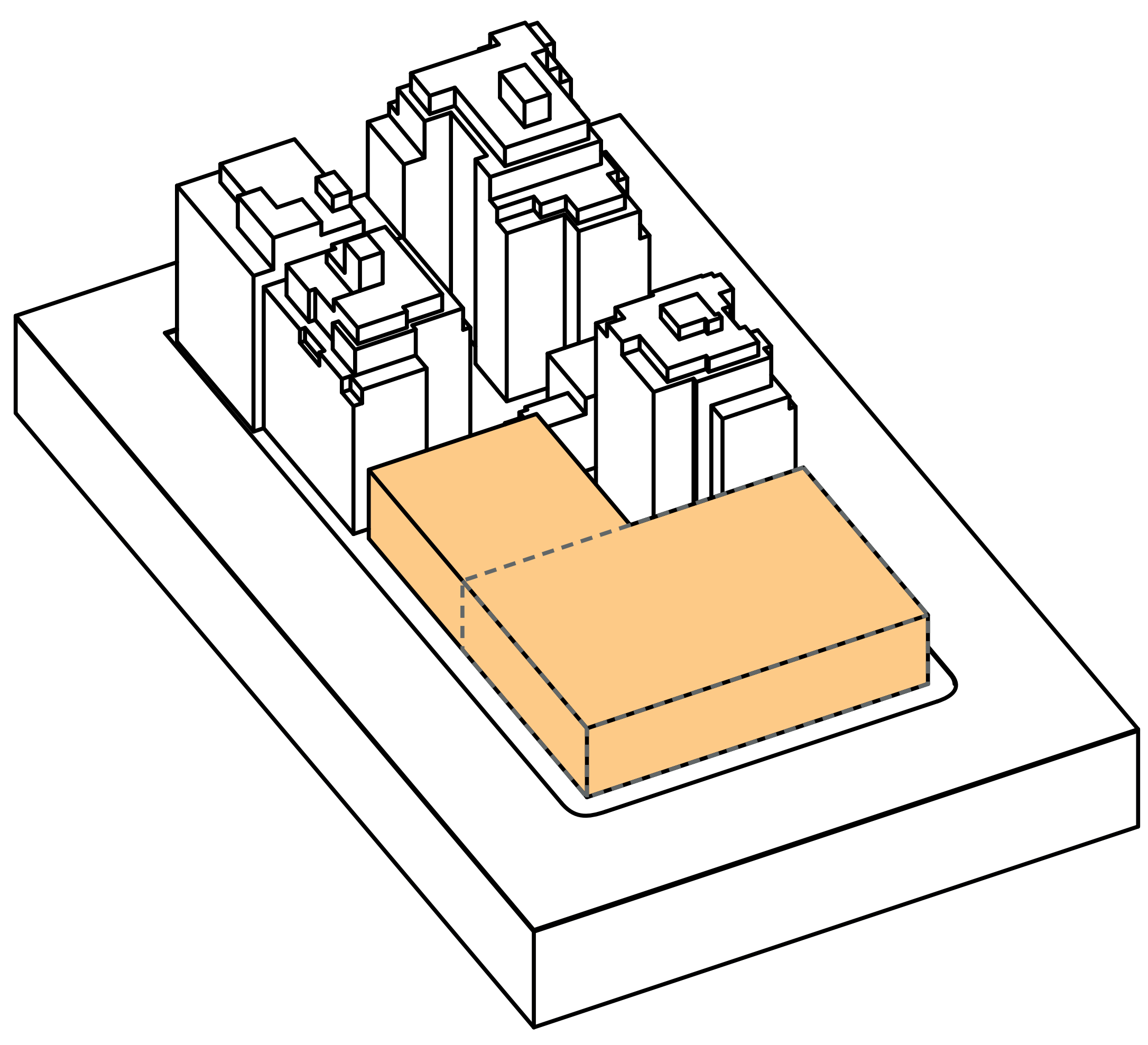




\section{Market hall \& Service space}

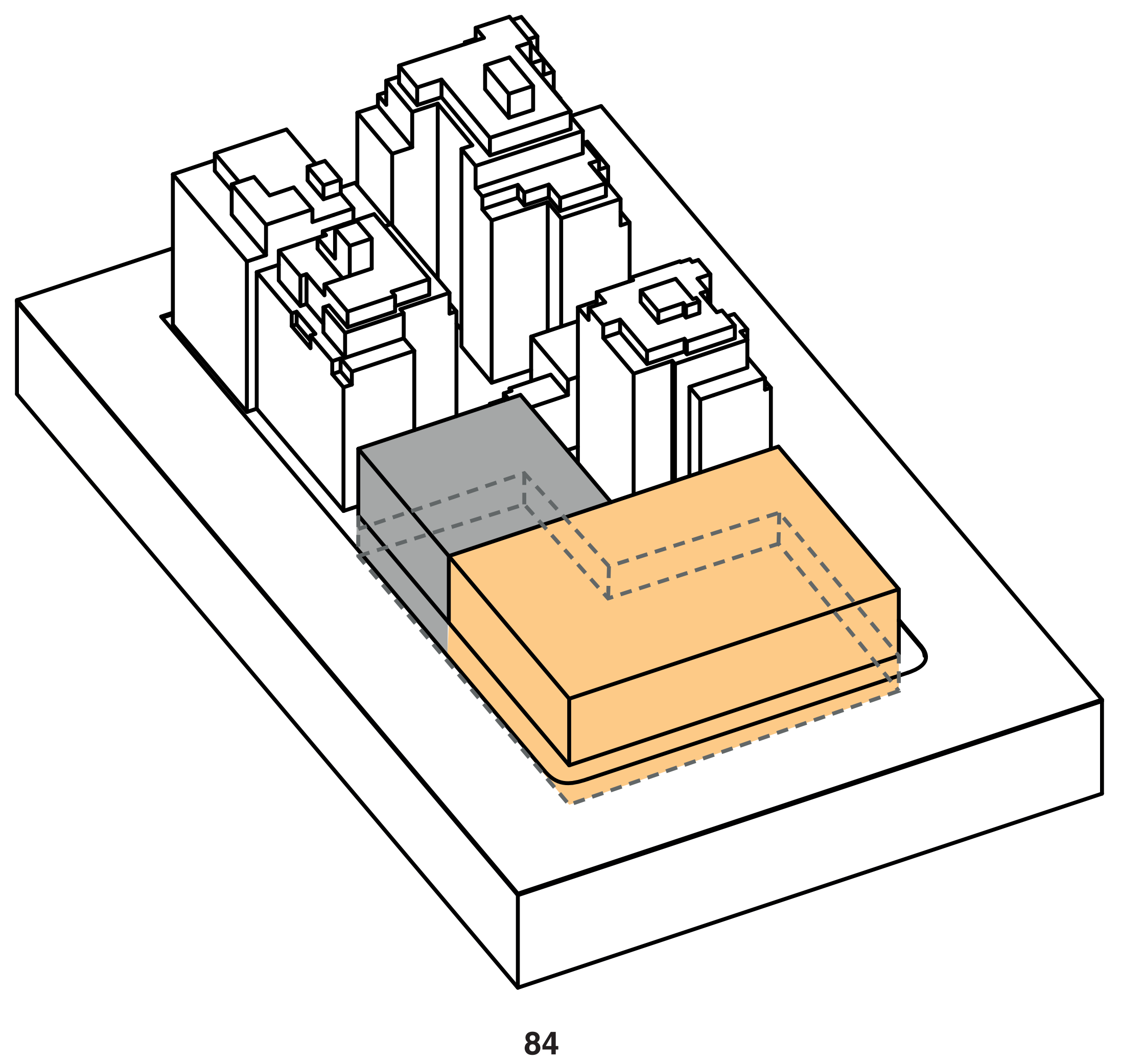




\section{Staggered floor}

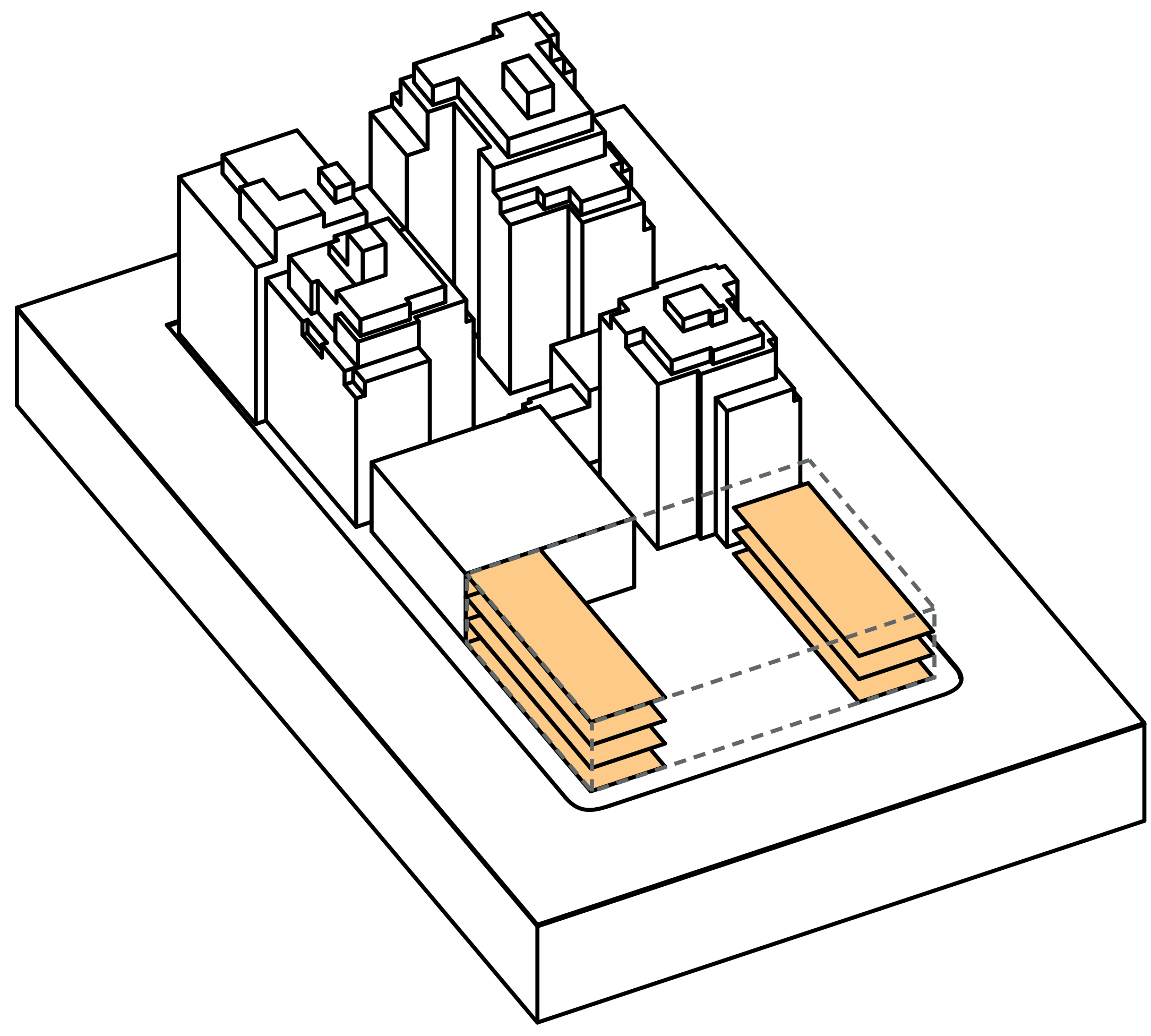




\section{Staggered floor}

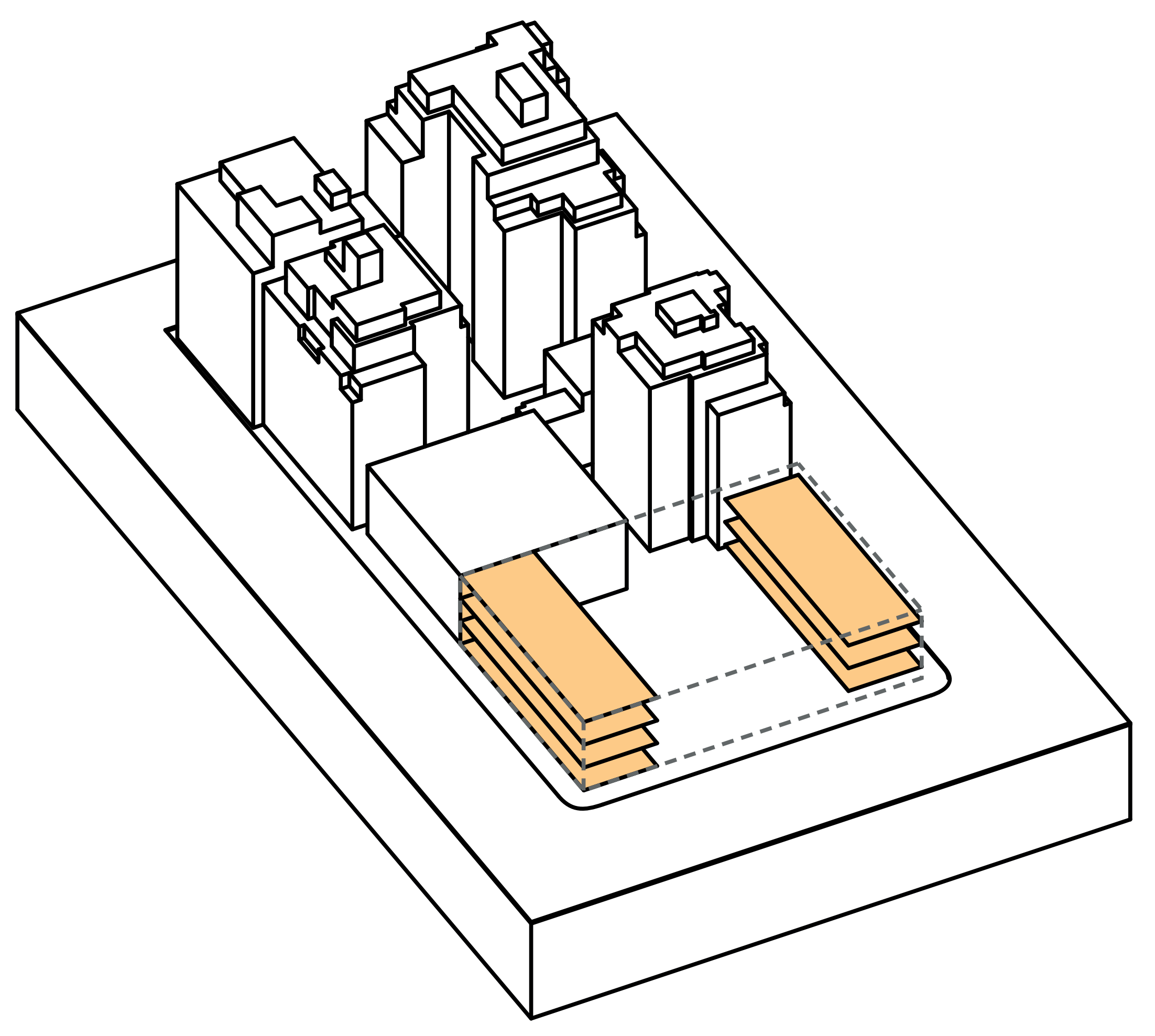


Interior \& external ramps

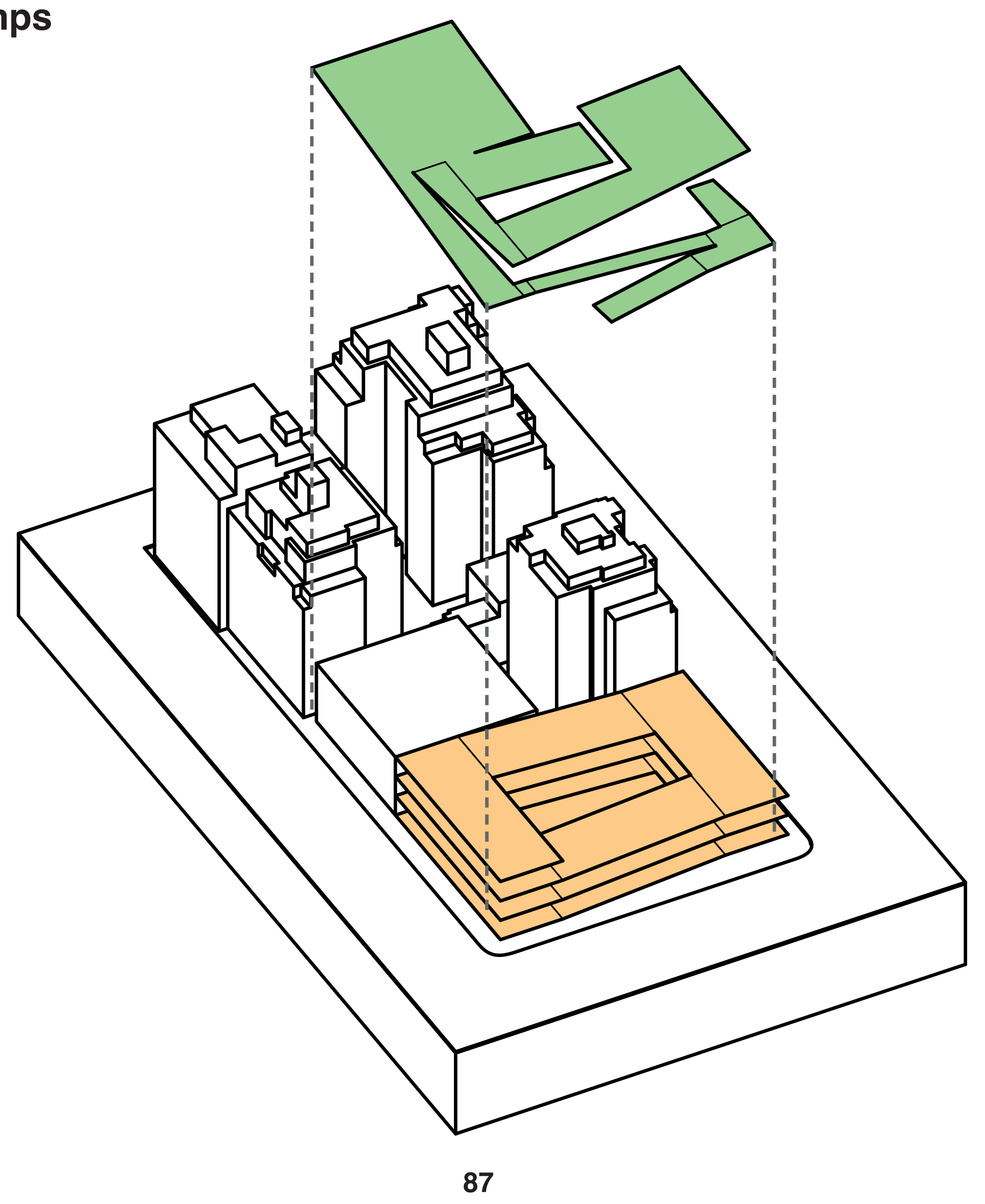


Interior \& external ramps

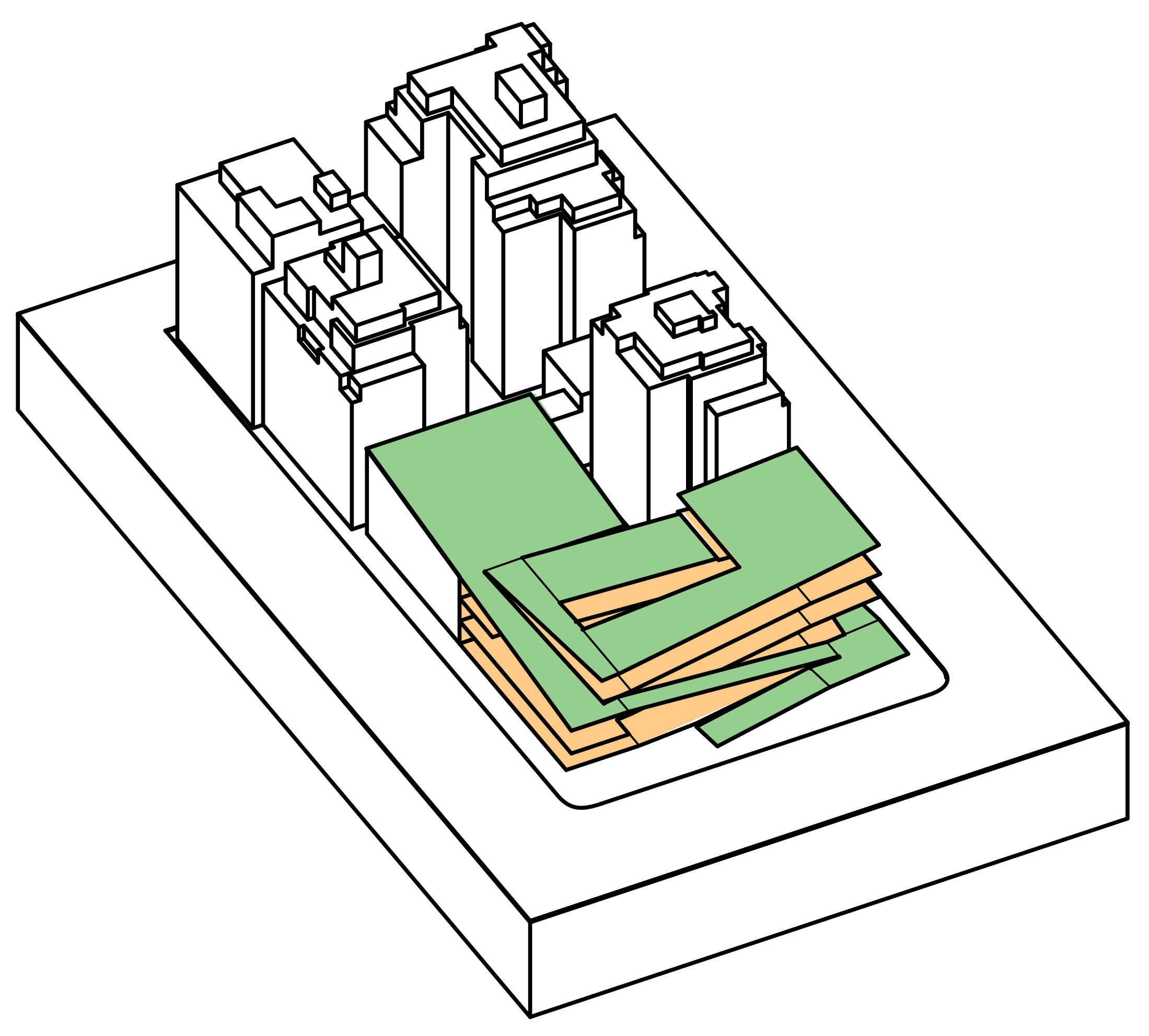


Residential tower

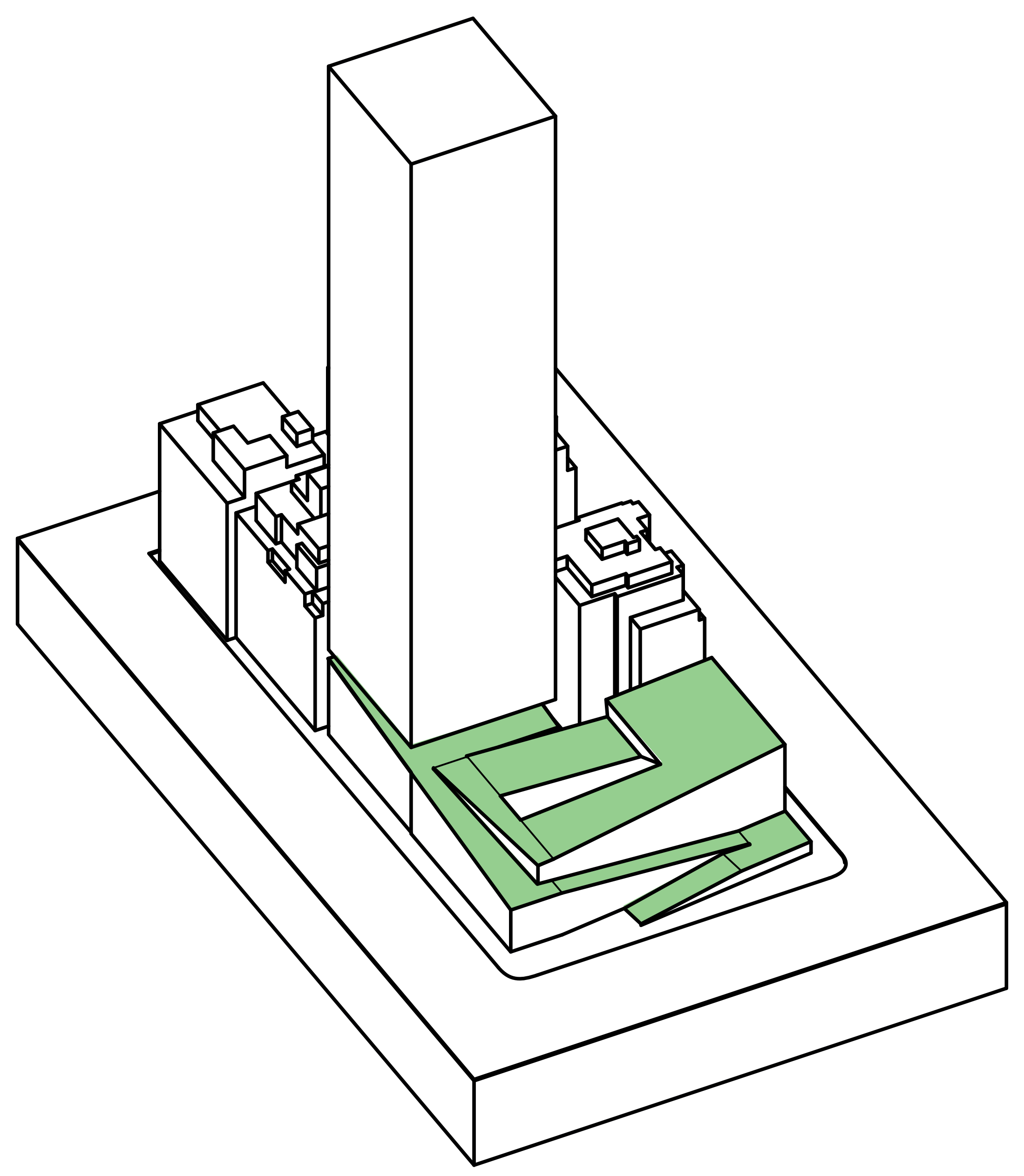


A recreational experience
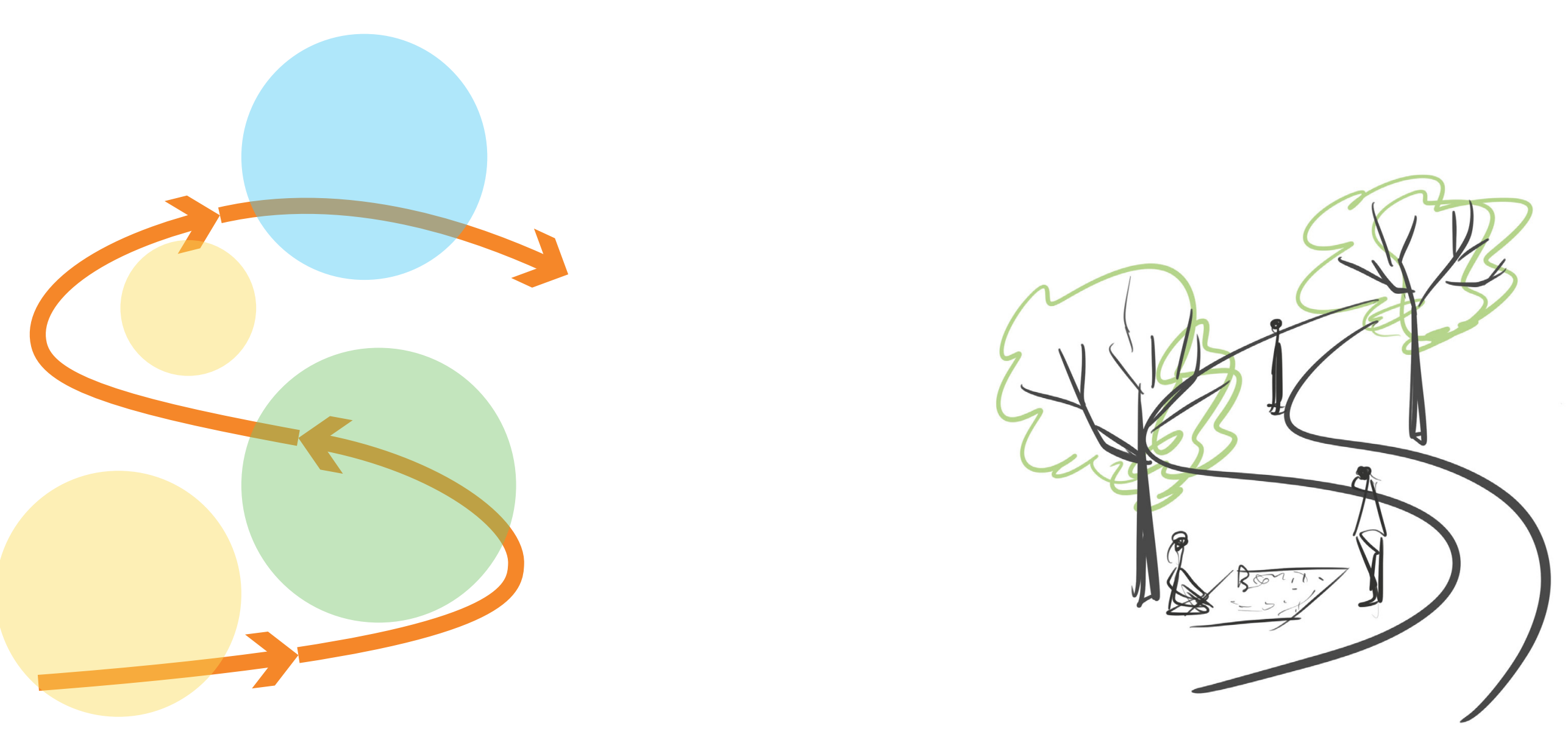

90 

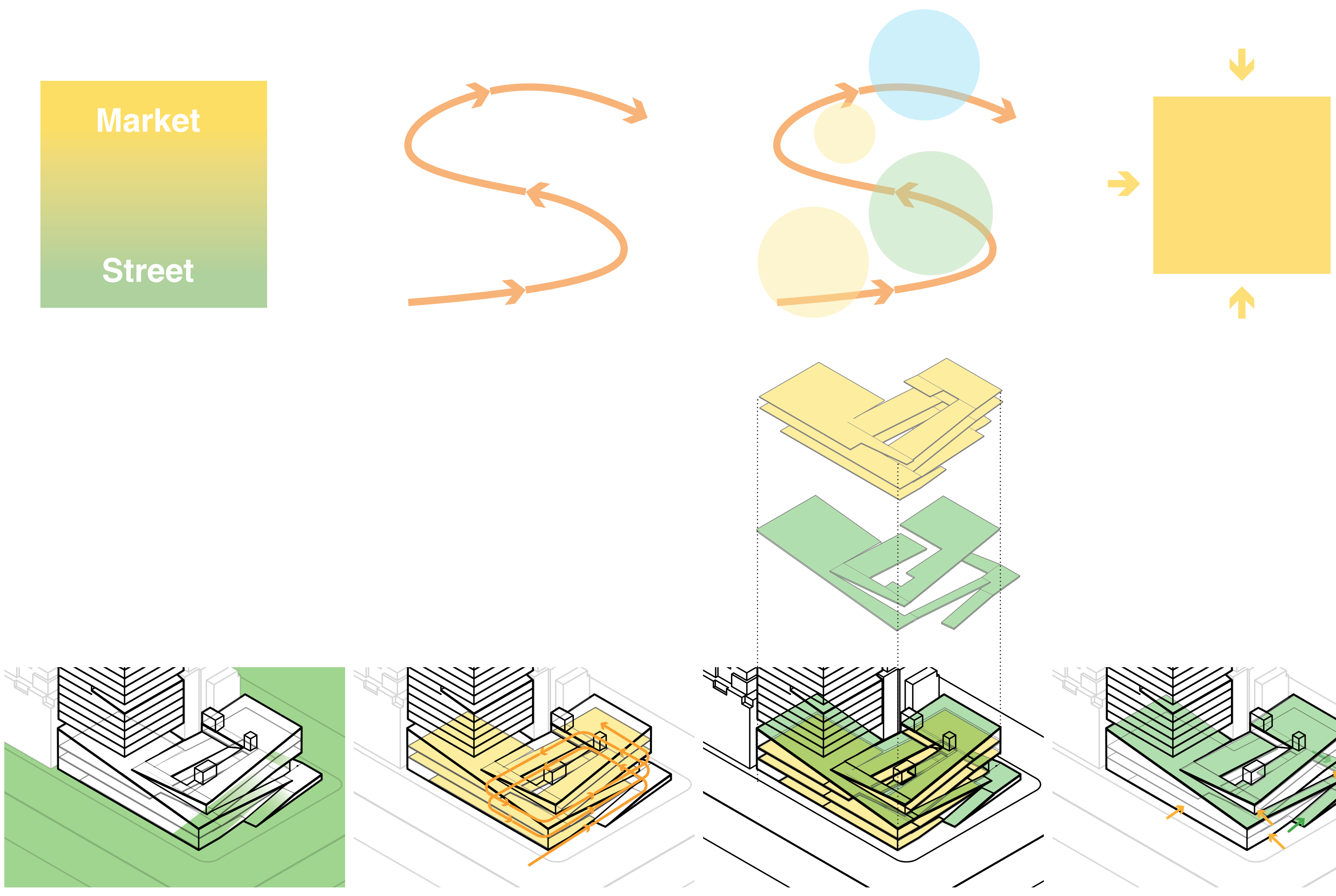

Publicity

Verticality Recreation

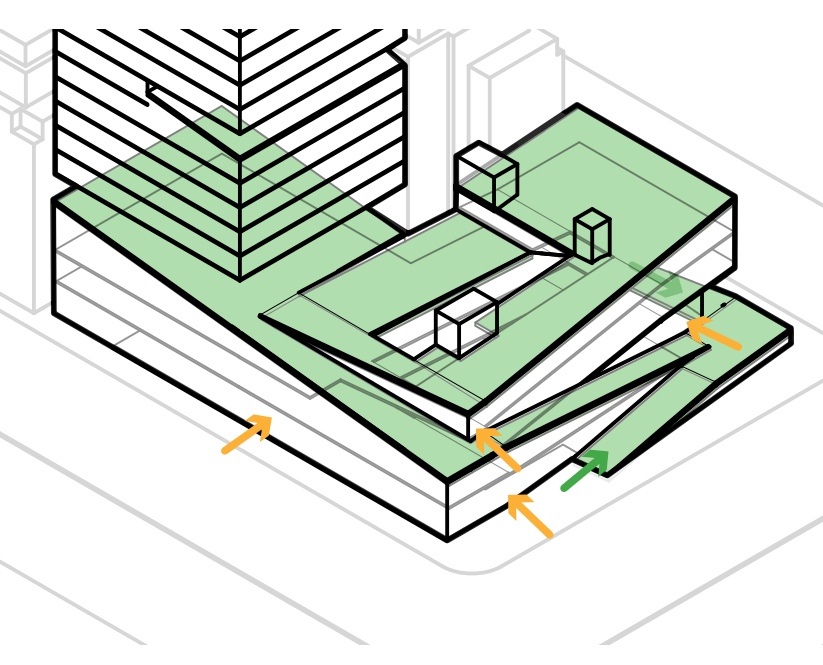

Accessiblity 


\section{Authentic New York Shopping Experience}

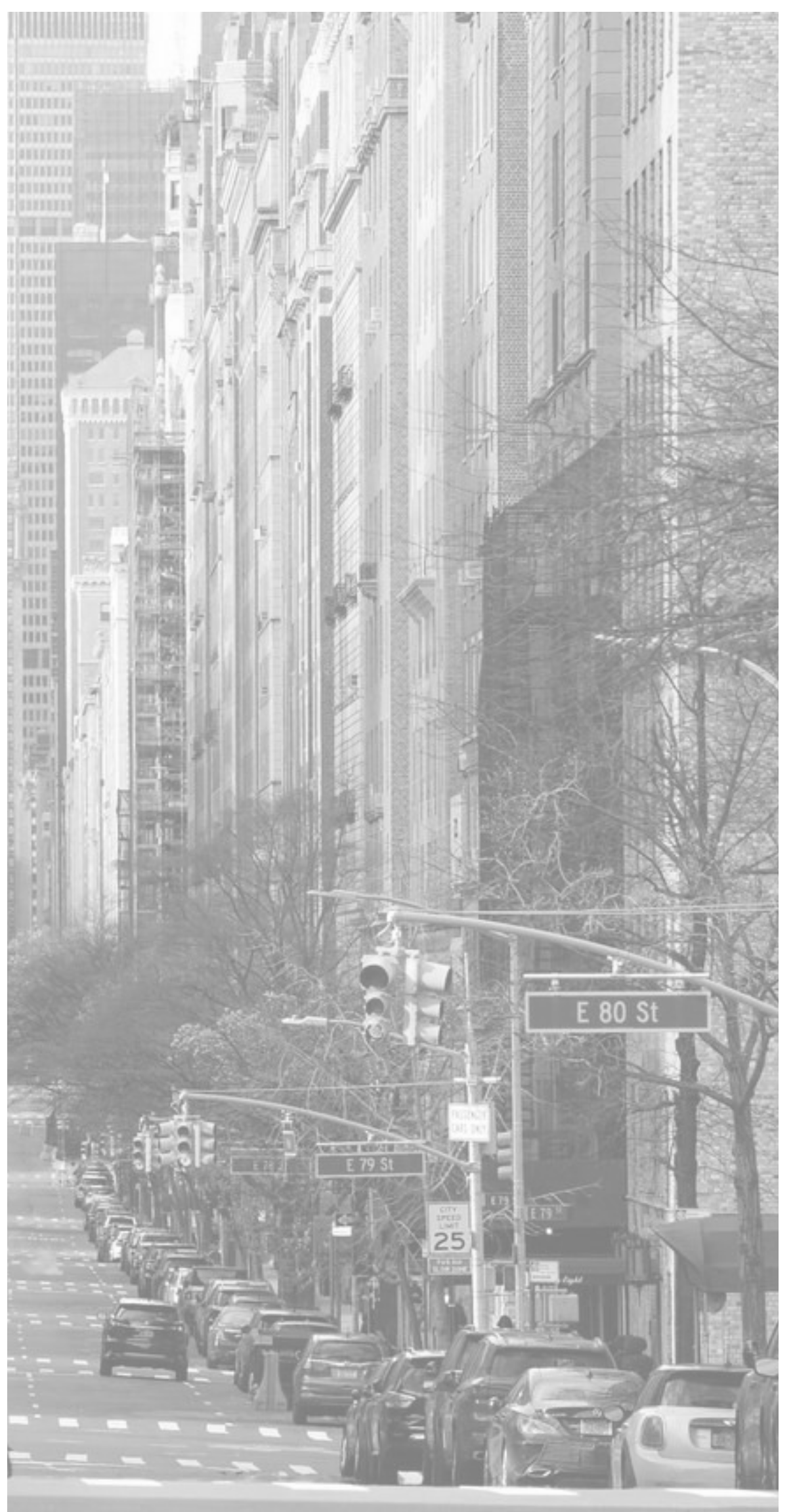

Verticality Busy Diversity

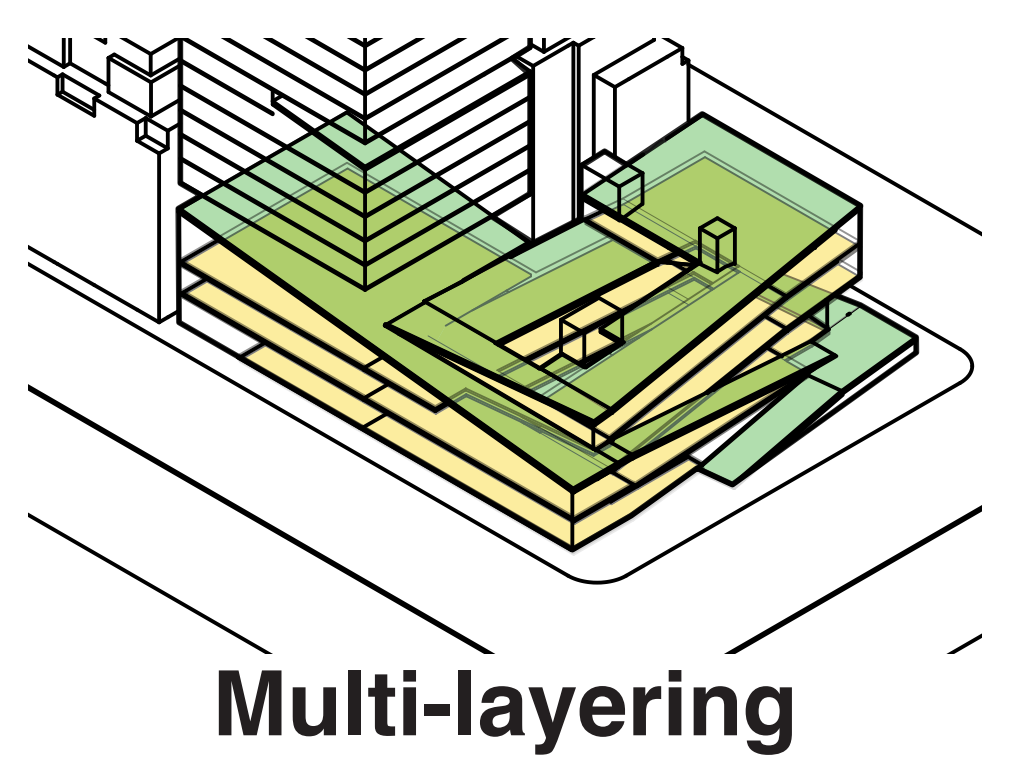

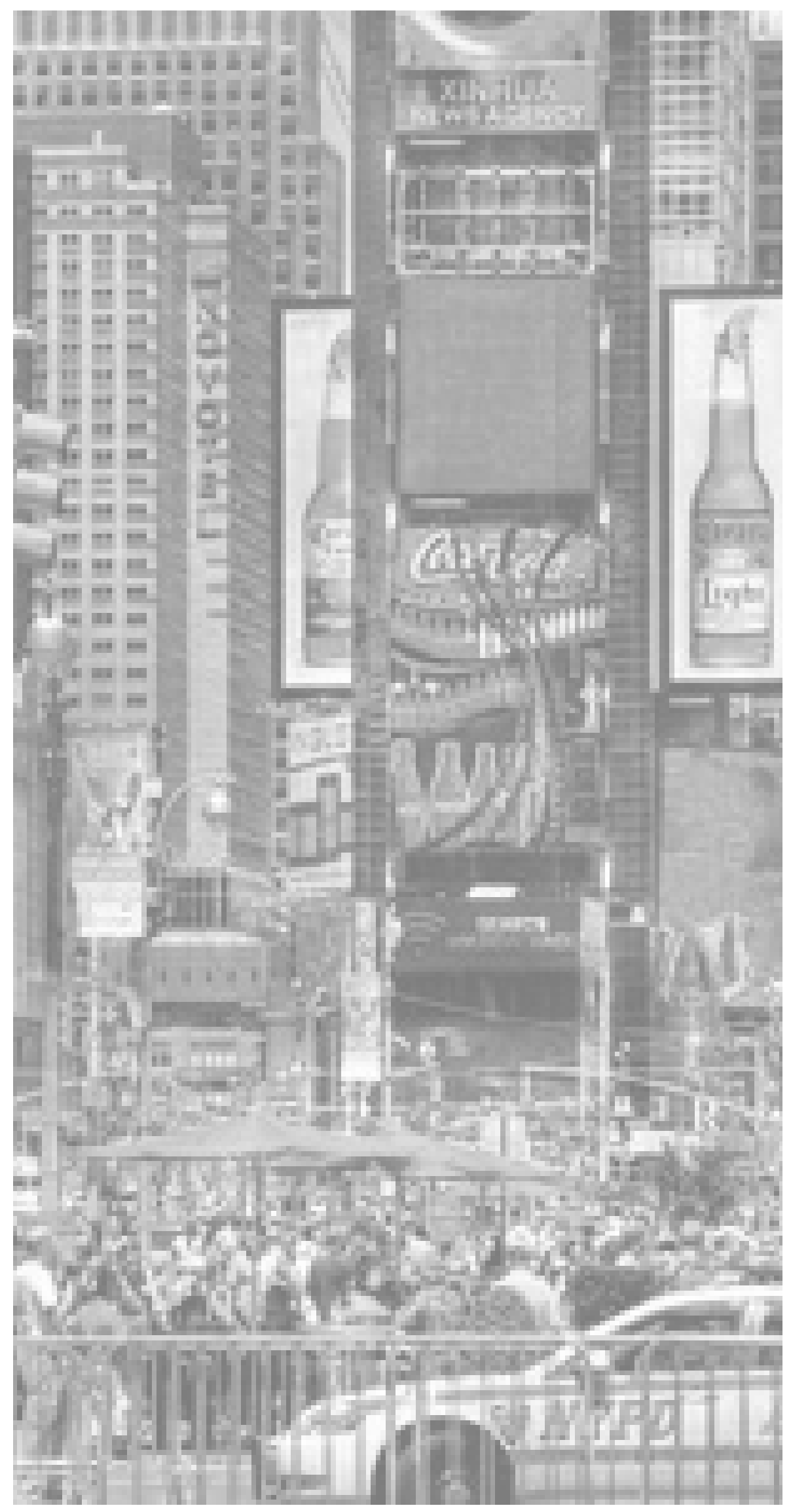




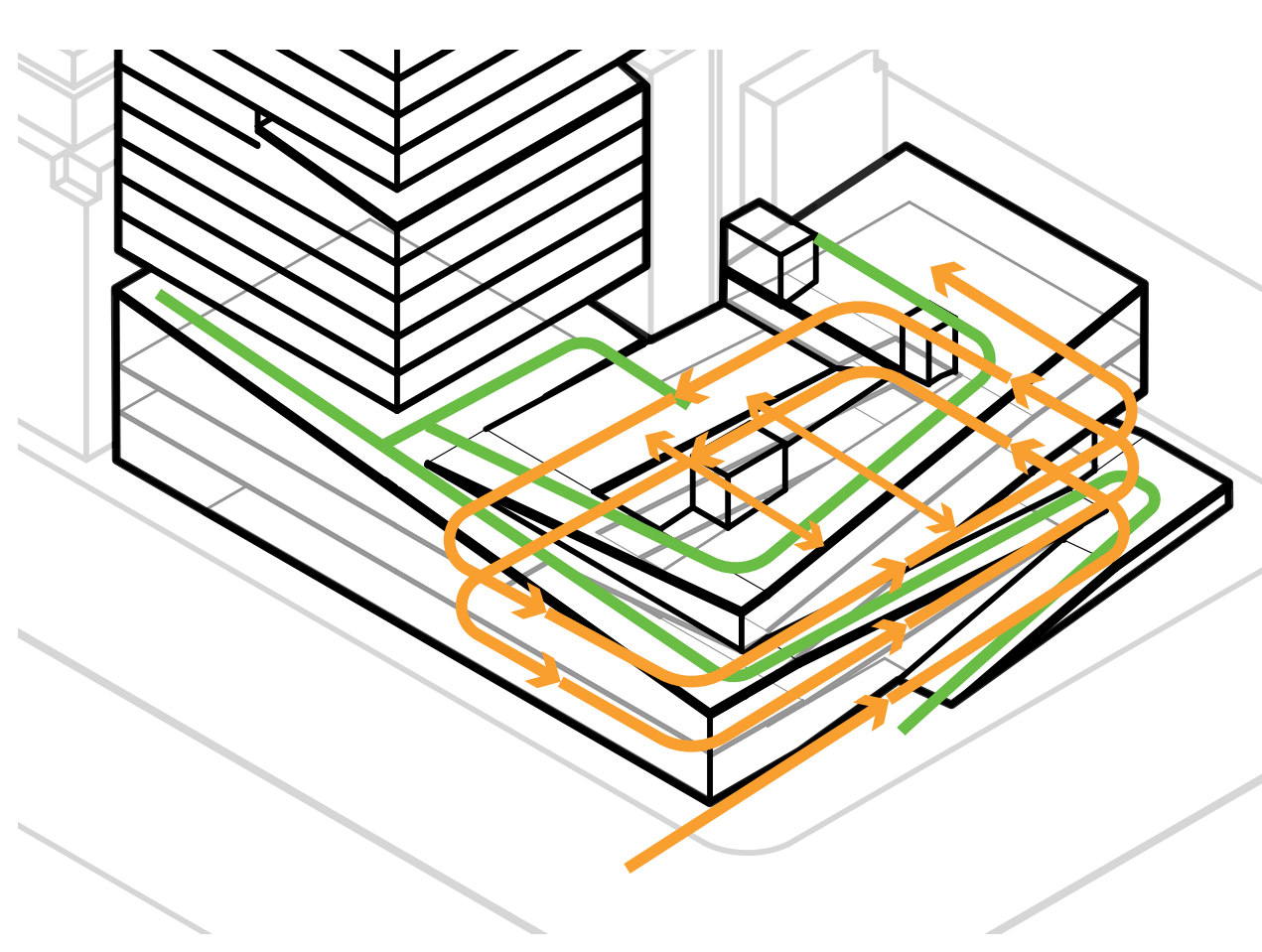

layers of circulation

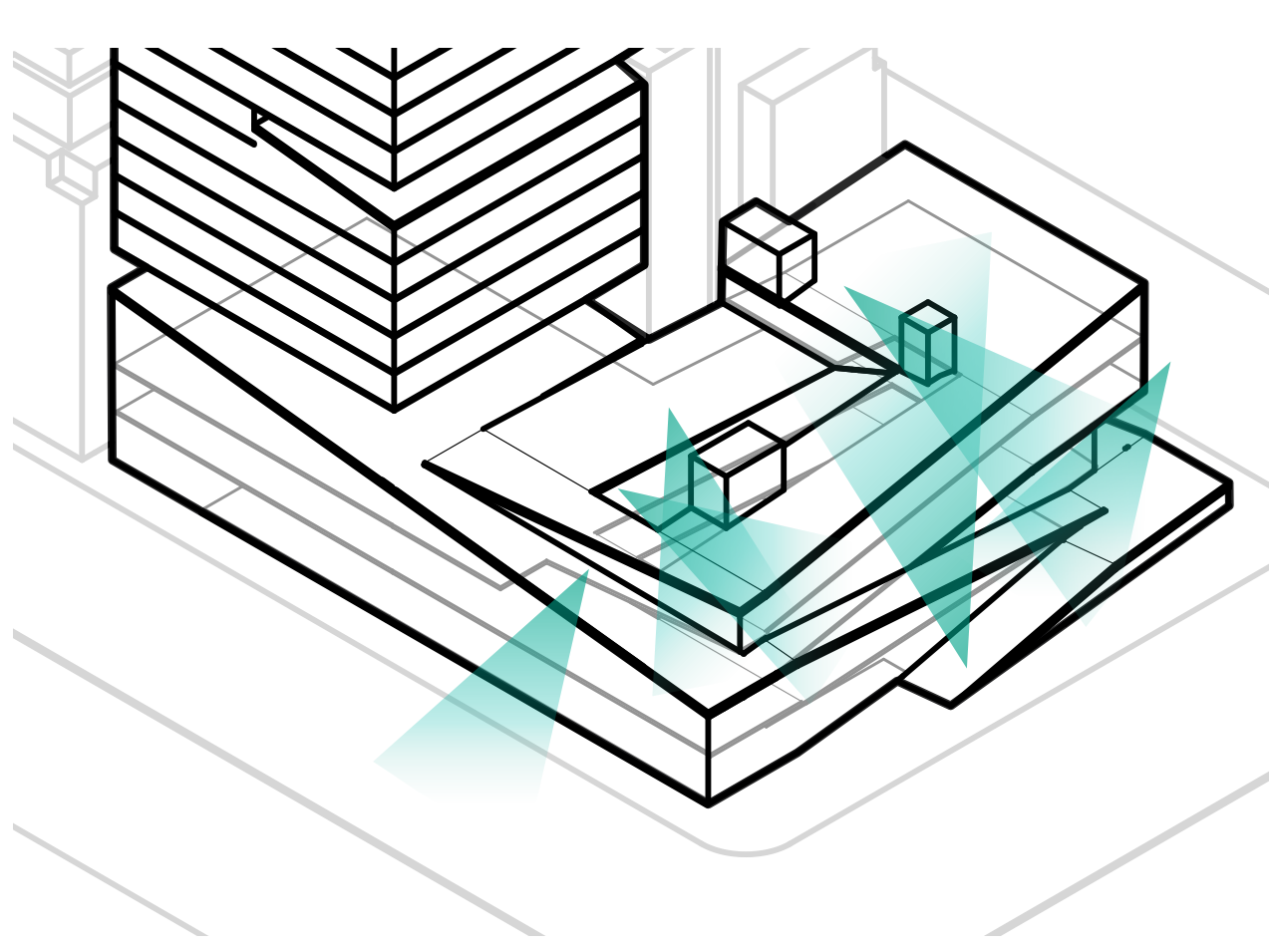

layers of views

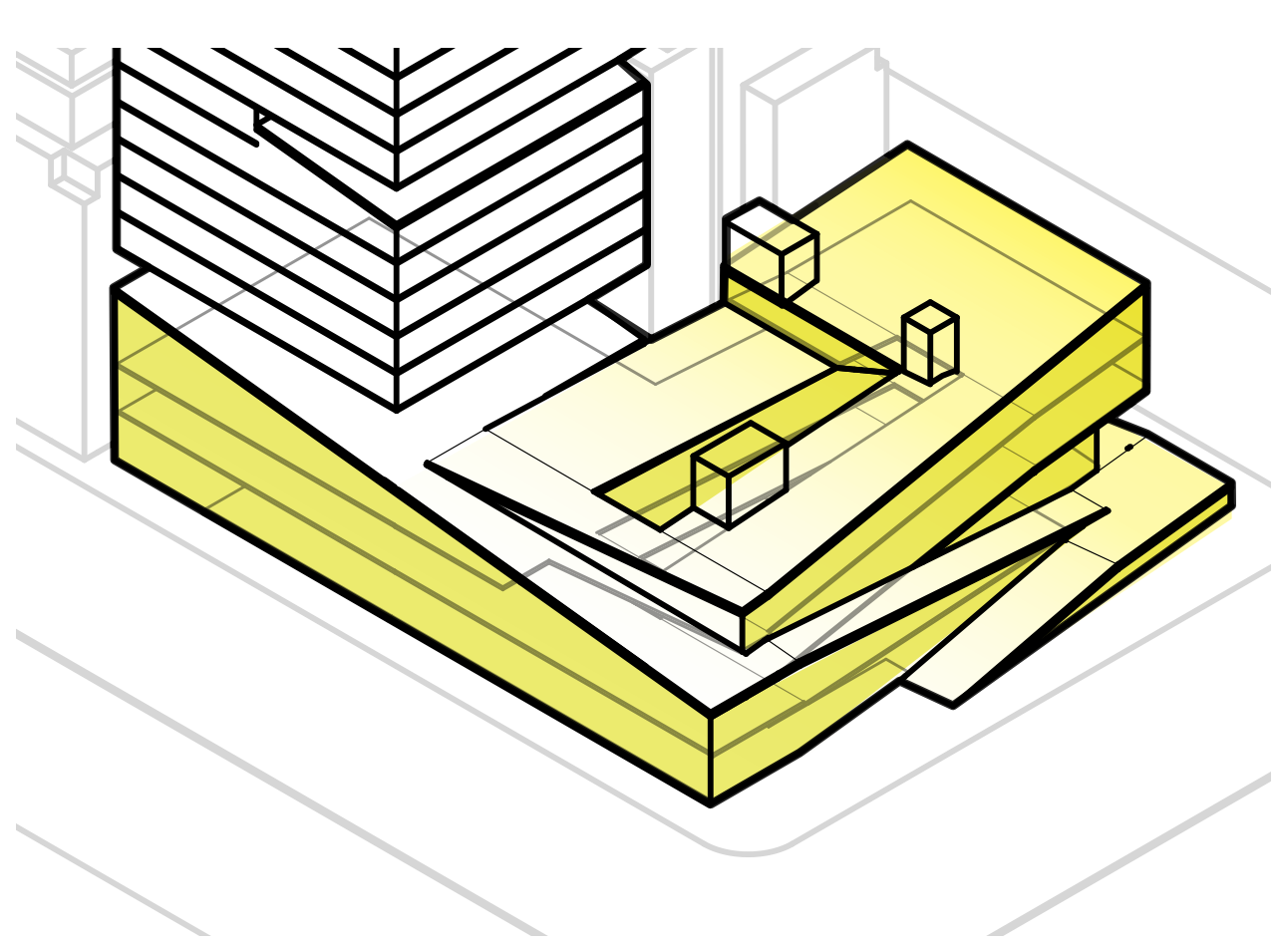

layers of natural lights 


\section{Authentic New York Shopping Experience}

Fresh local groceries sold by local farmers

Local people come here to shop groceries and meet with each other

No big brands/international chain stores

Casual, temporary, flexible, spontaneous 

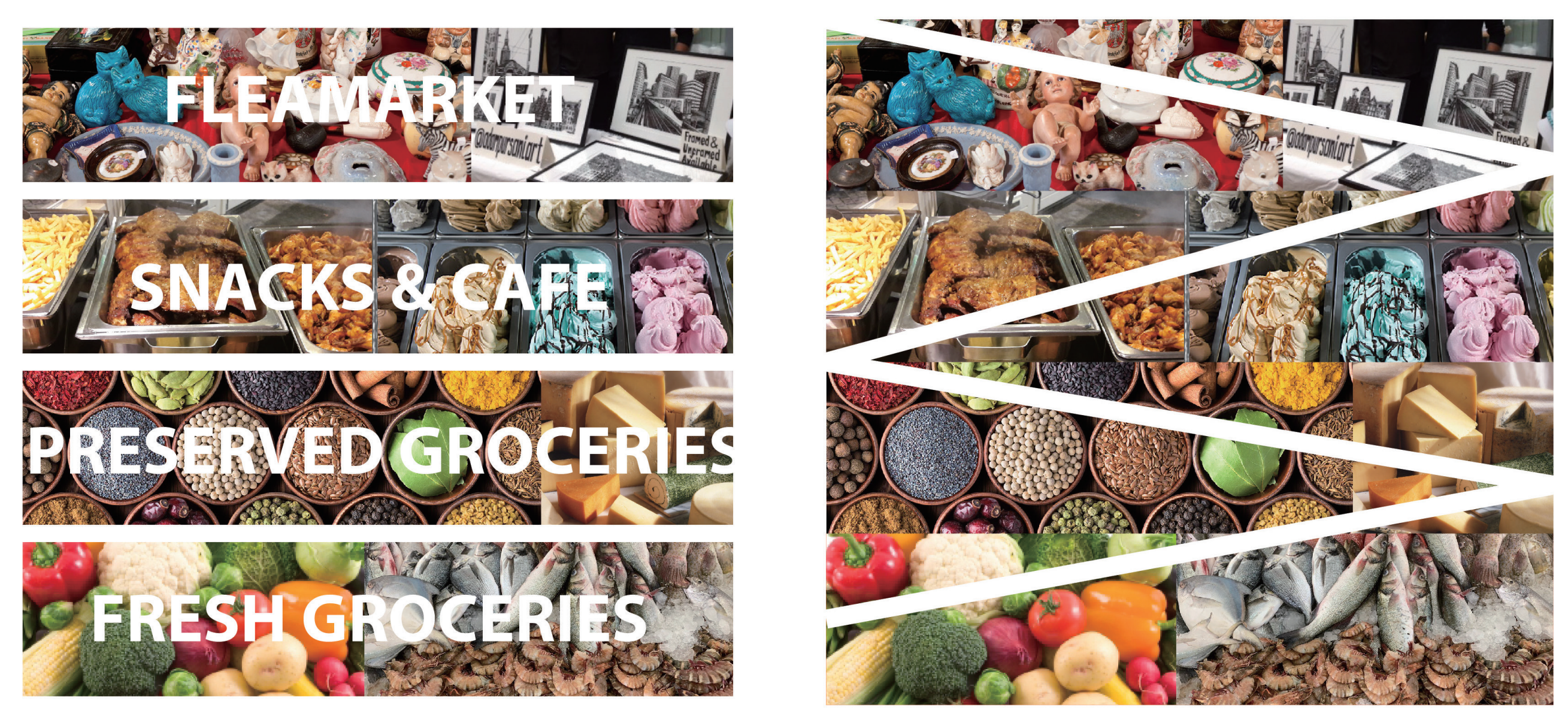


\section{SMALL}

STALLS

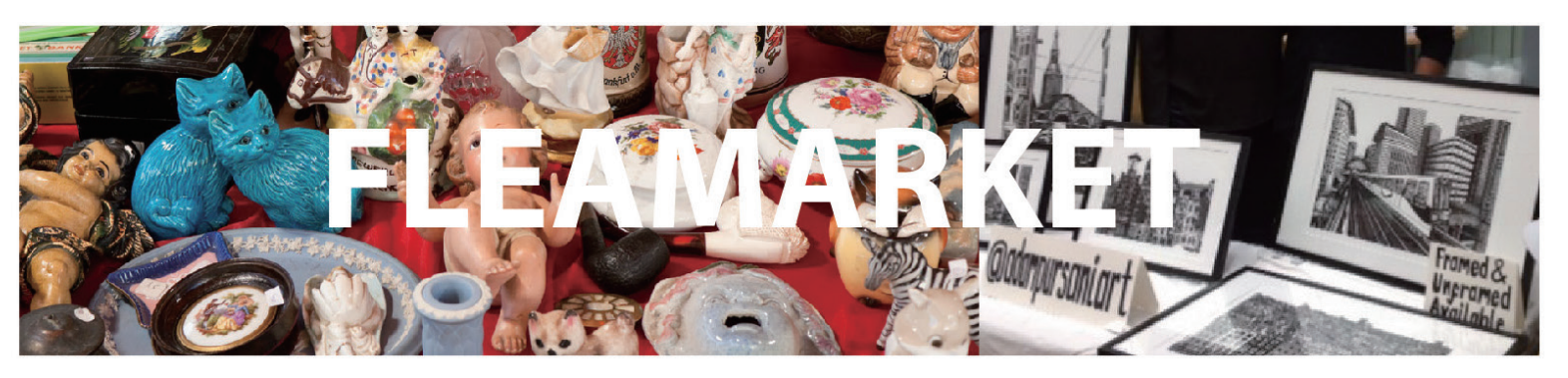

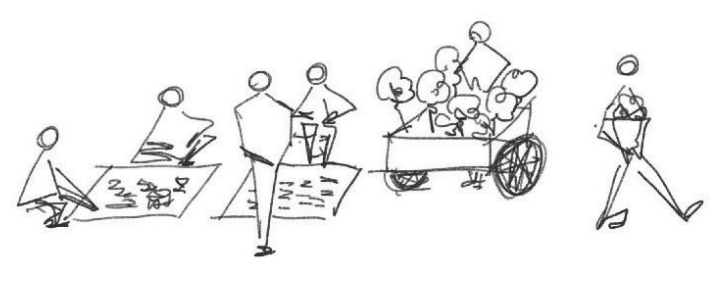
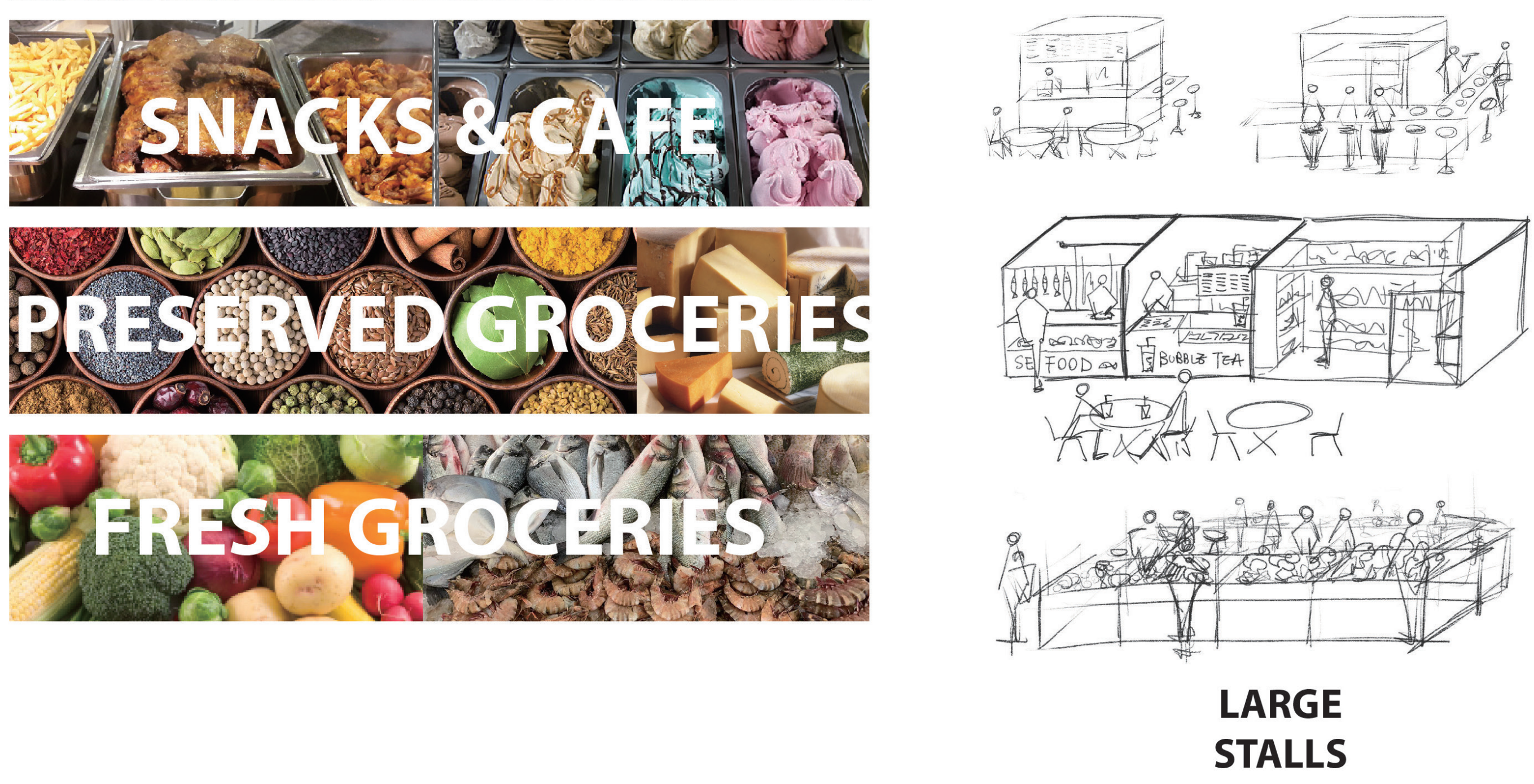


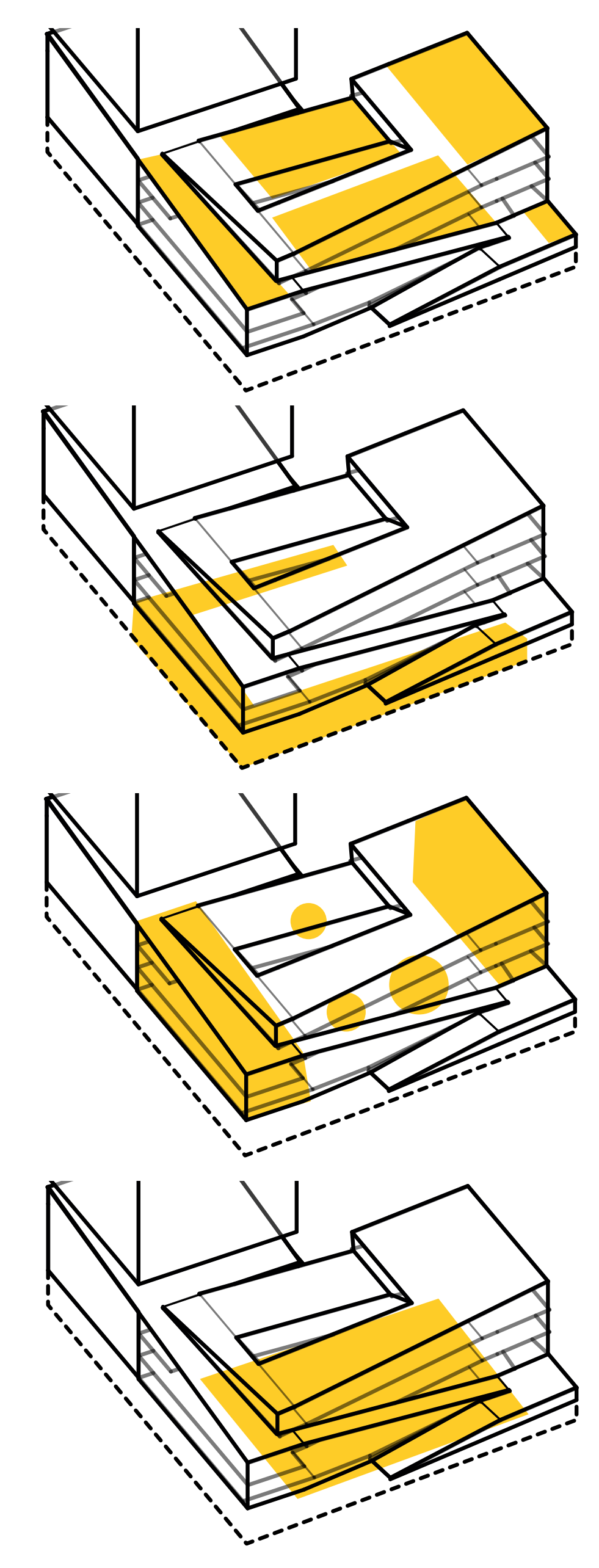

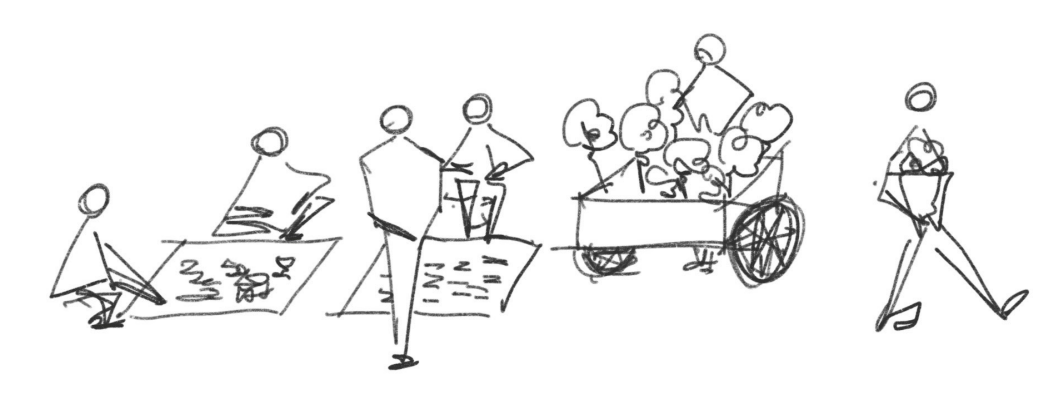

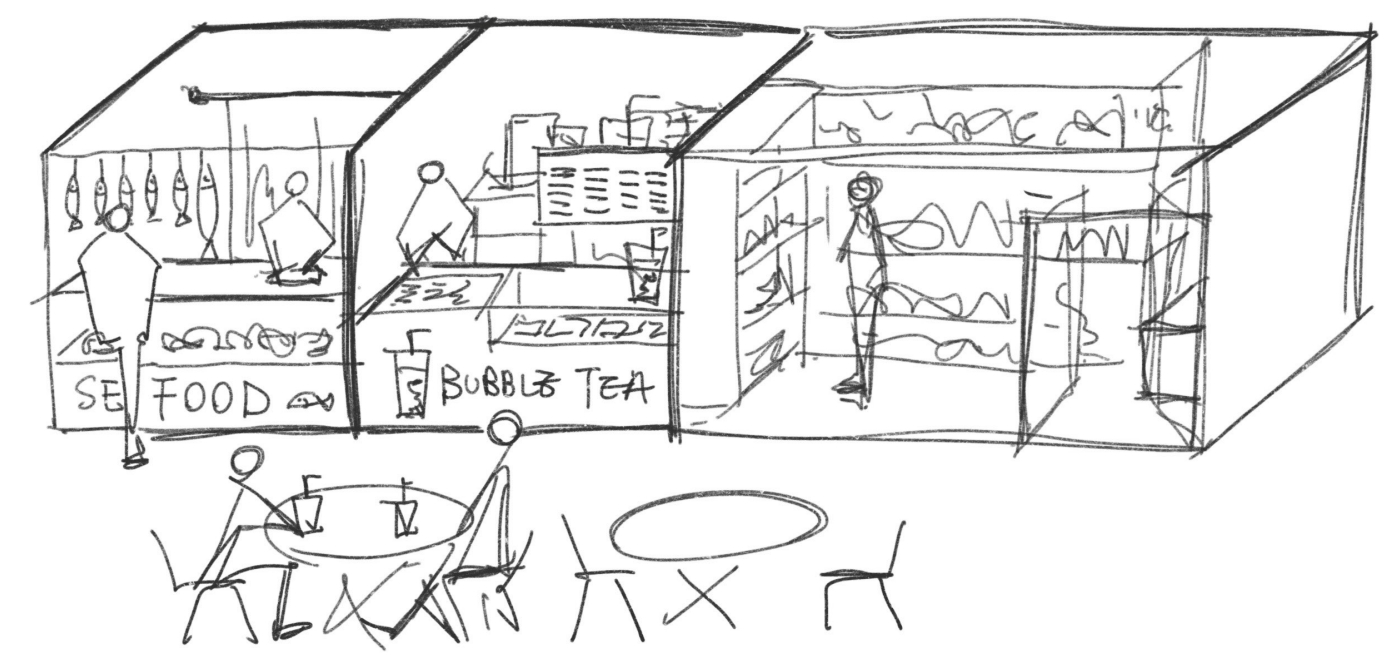

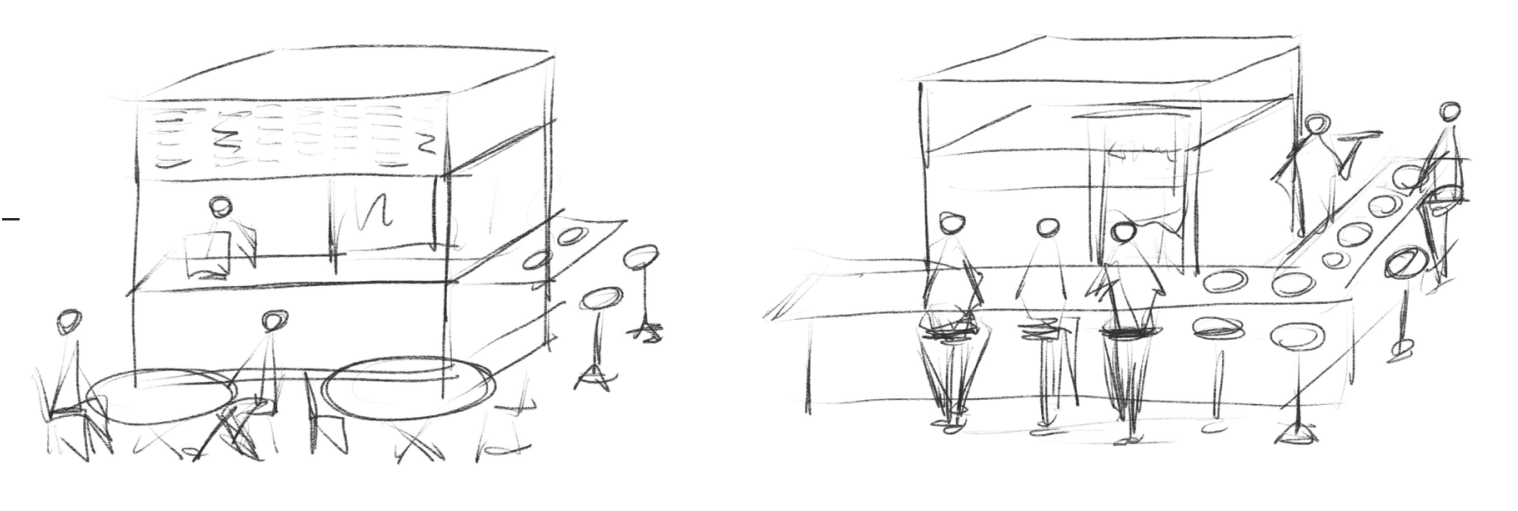

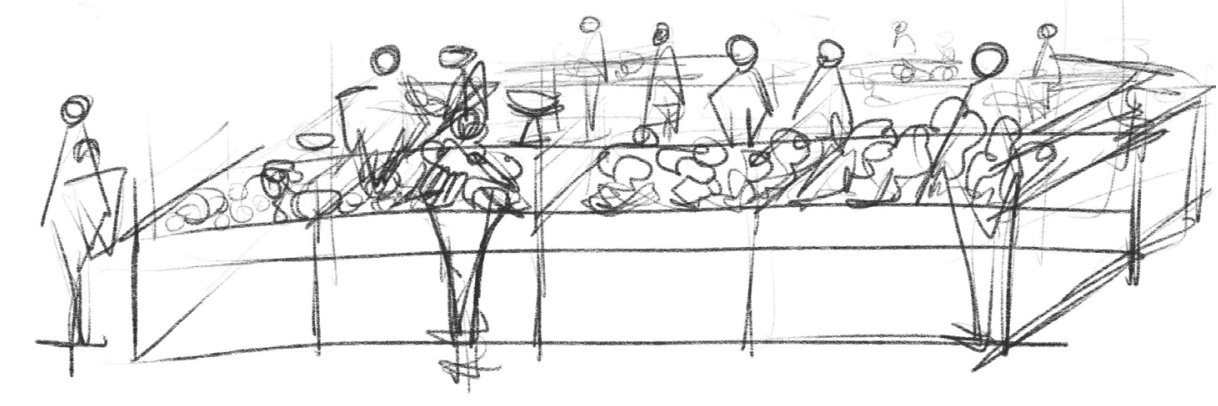




\section{Residential tower}

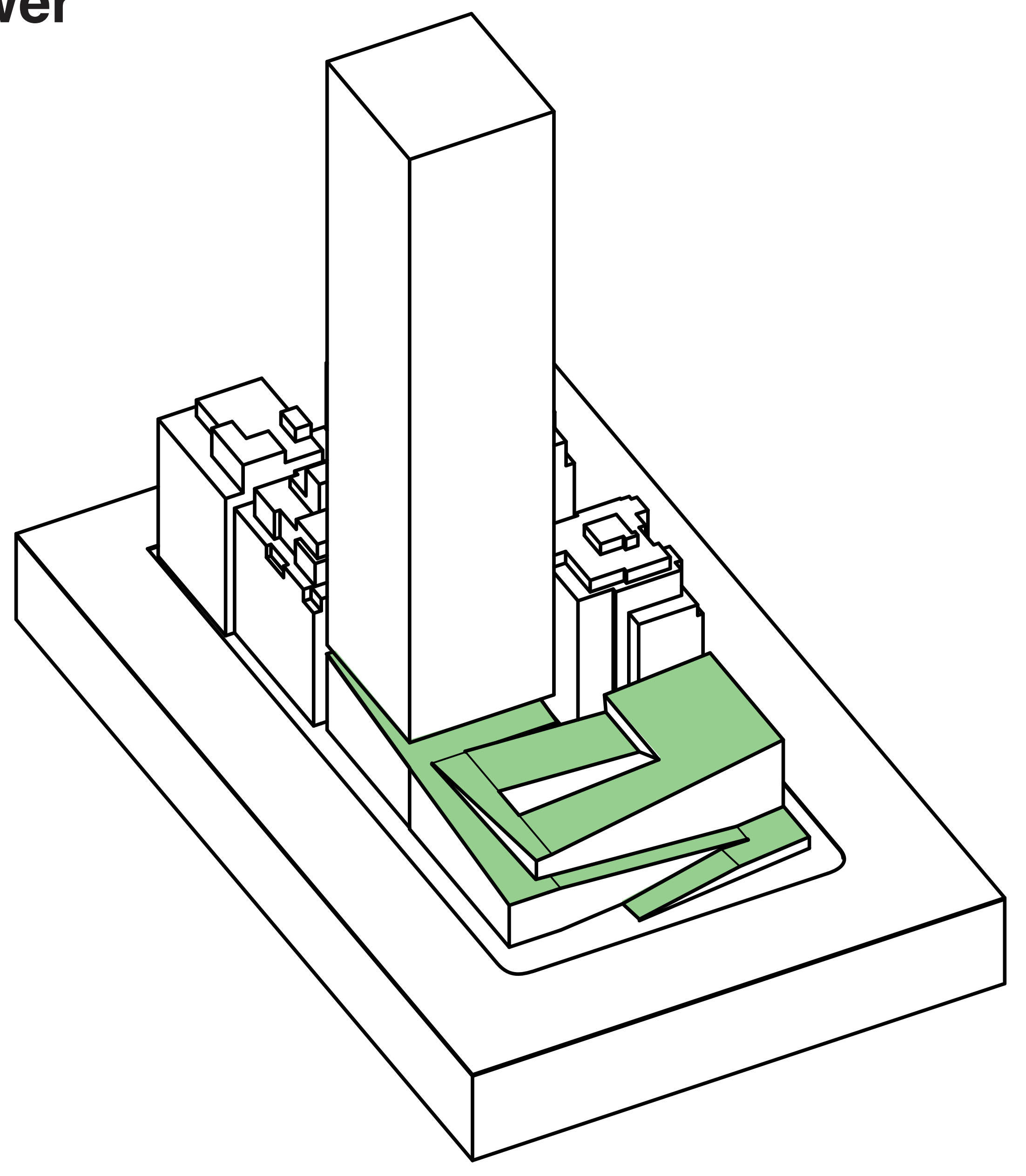




\section{Residential tower}

Communal garden for every 5 floors

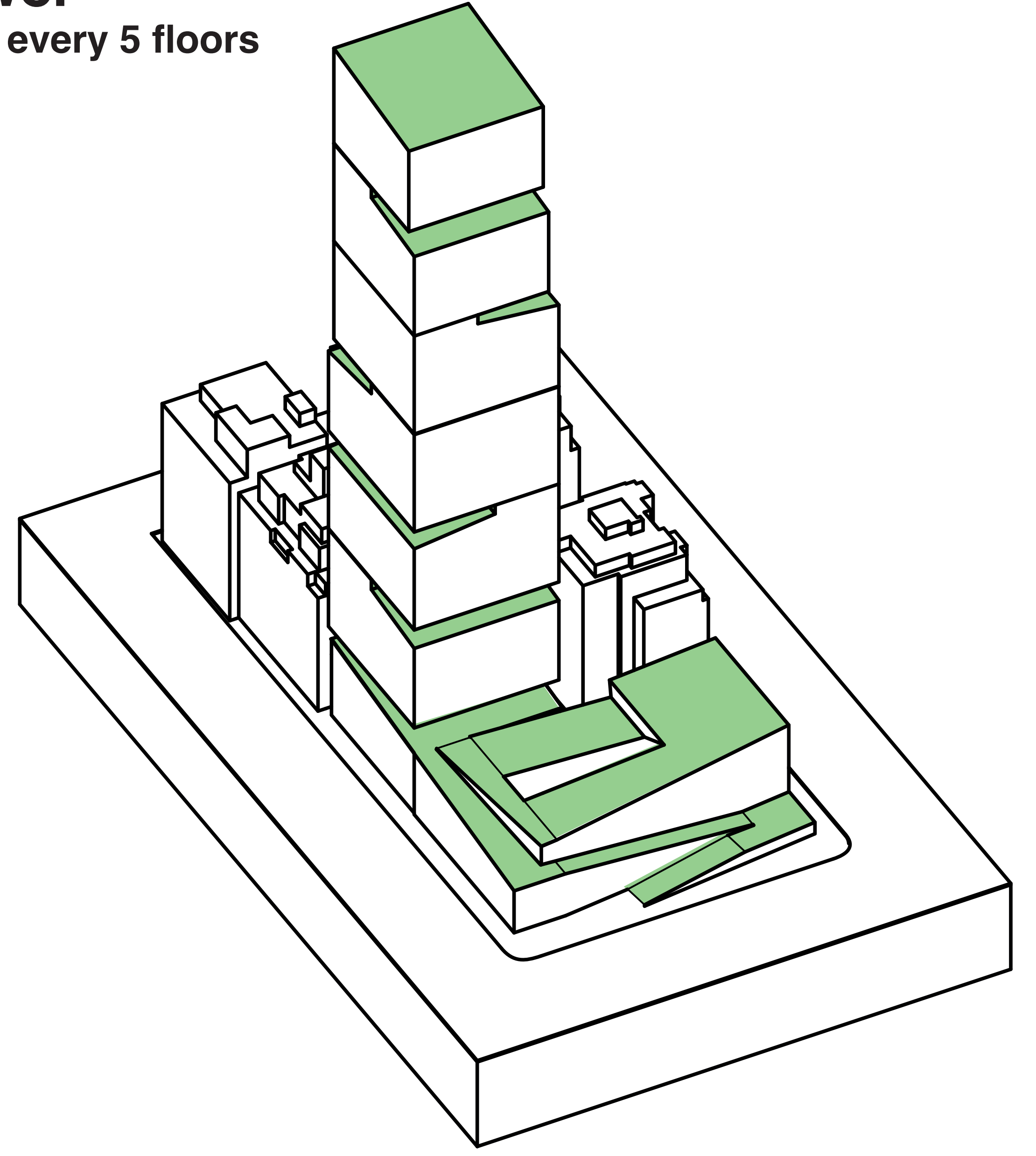


Plot size: $3,800 \mathrm{~m}^{2}$ Mareket: $11000 \mathrm{~m}^{2}$ Residential: $29000 \mathrm{~m}^{2}$ Public roofgarden: $3000 \mathrm{~m}^{2}$ Total GFA: $40000 \mathrm{~m}^{2}$

FAR: 10.5
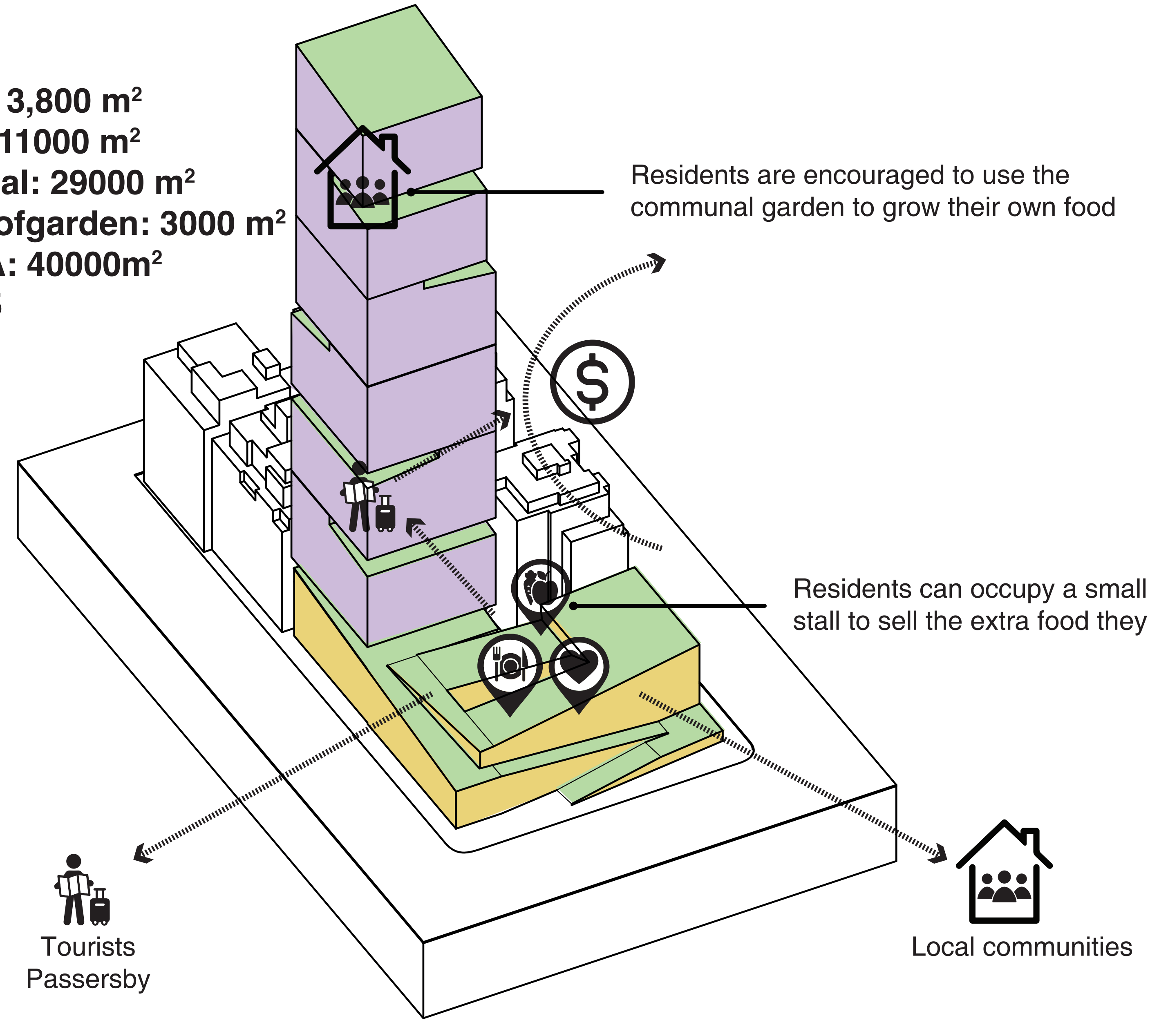

Residents are encouraged to use the communal garden to grow their own food 


\section{Design Implementation}




\section{Group Masterplan}

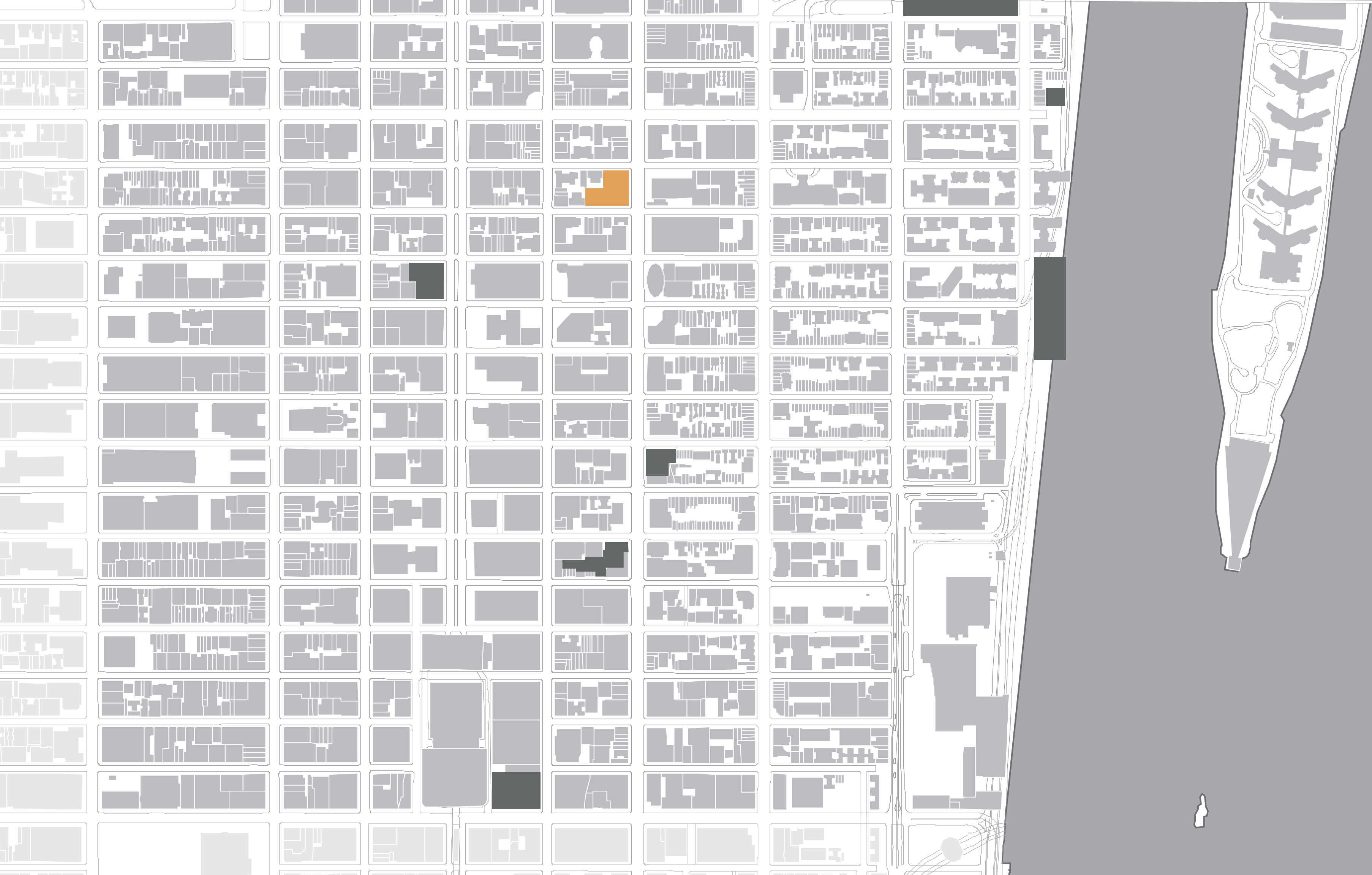

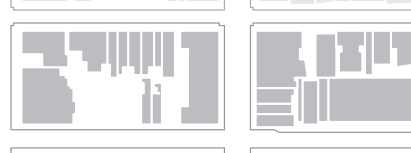

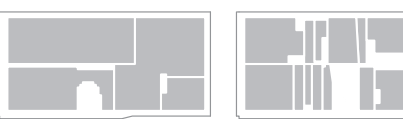

플

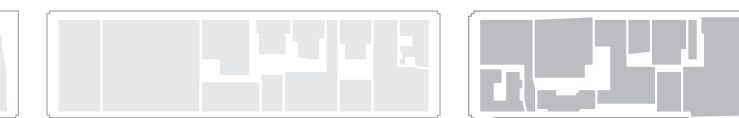

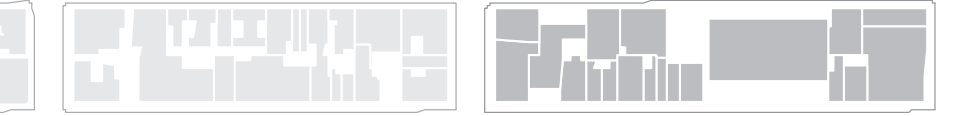

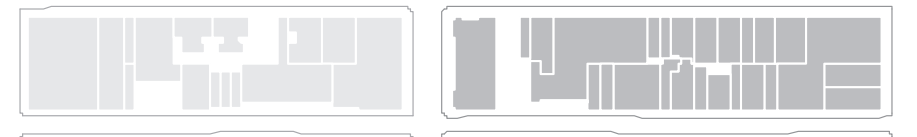

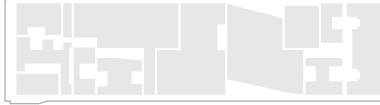

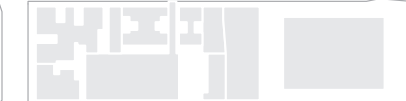

$+11+$

$+2$

(2)
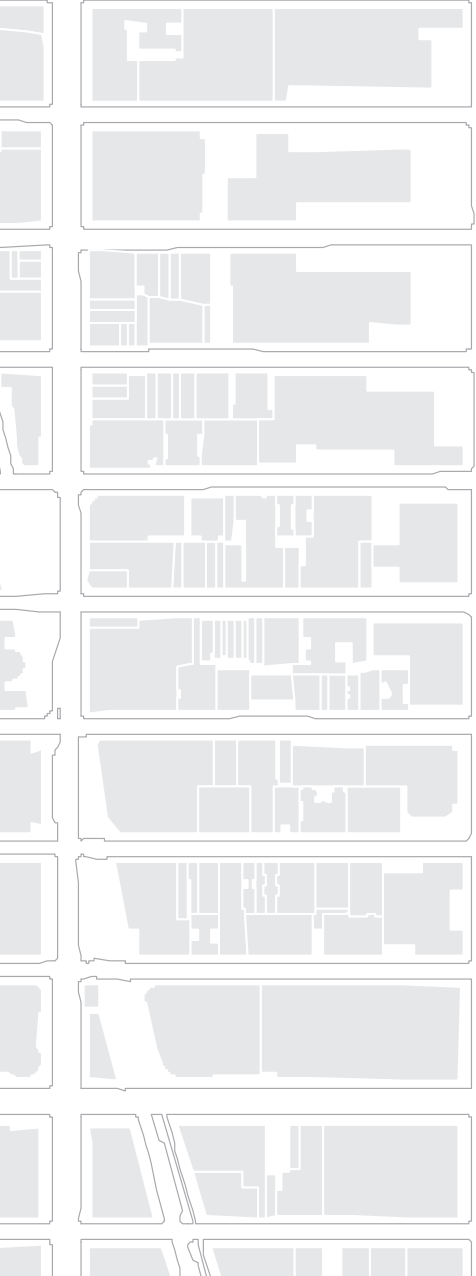

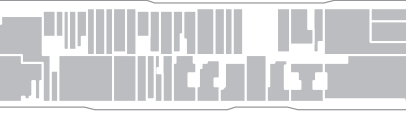

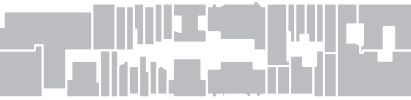

Ii i n

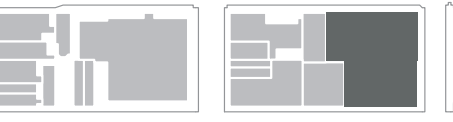

$=-1+\square$

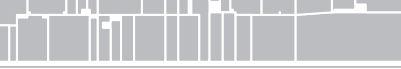

Finilini

10 0

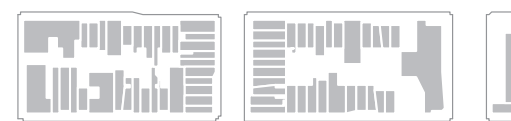

(1)

\section{I}

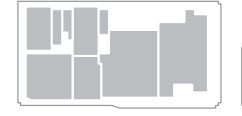

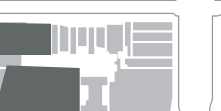

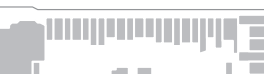

- III - IIII)

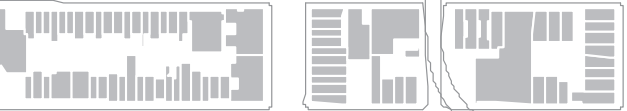

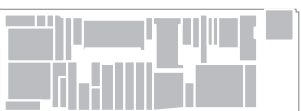

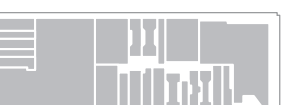

$r \sqrt{n+10}$

매

1ili)

年

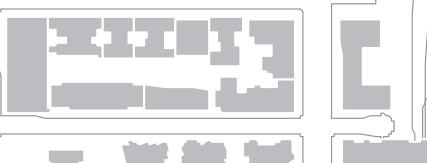

드논

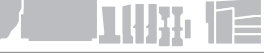

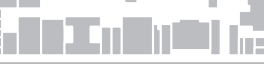

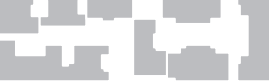

Fant

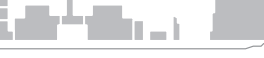

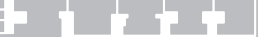

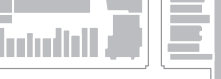

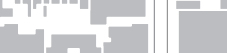




\section{Urban implementation}
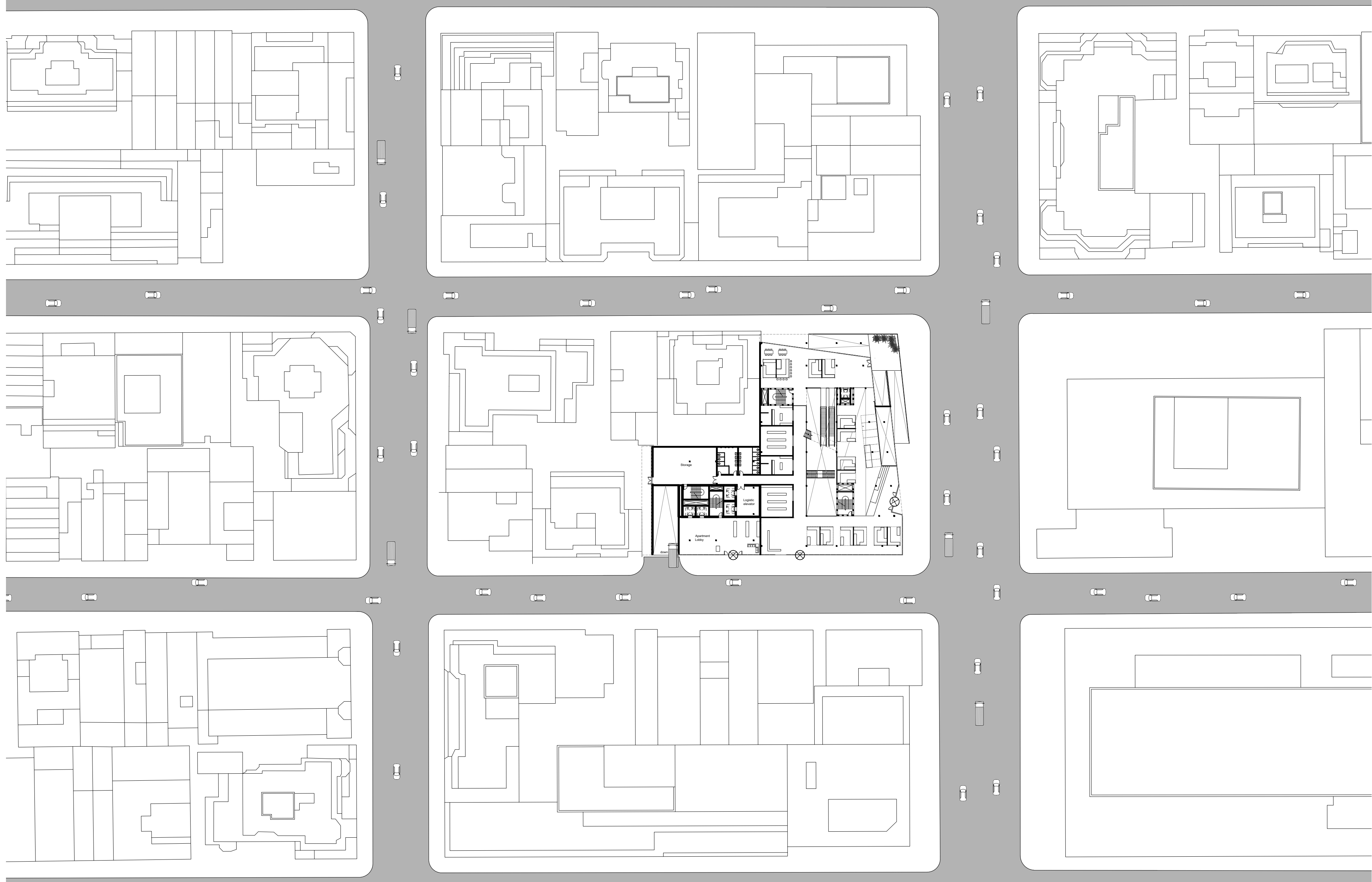

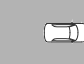




\section{Ground Floor}
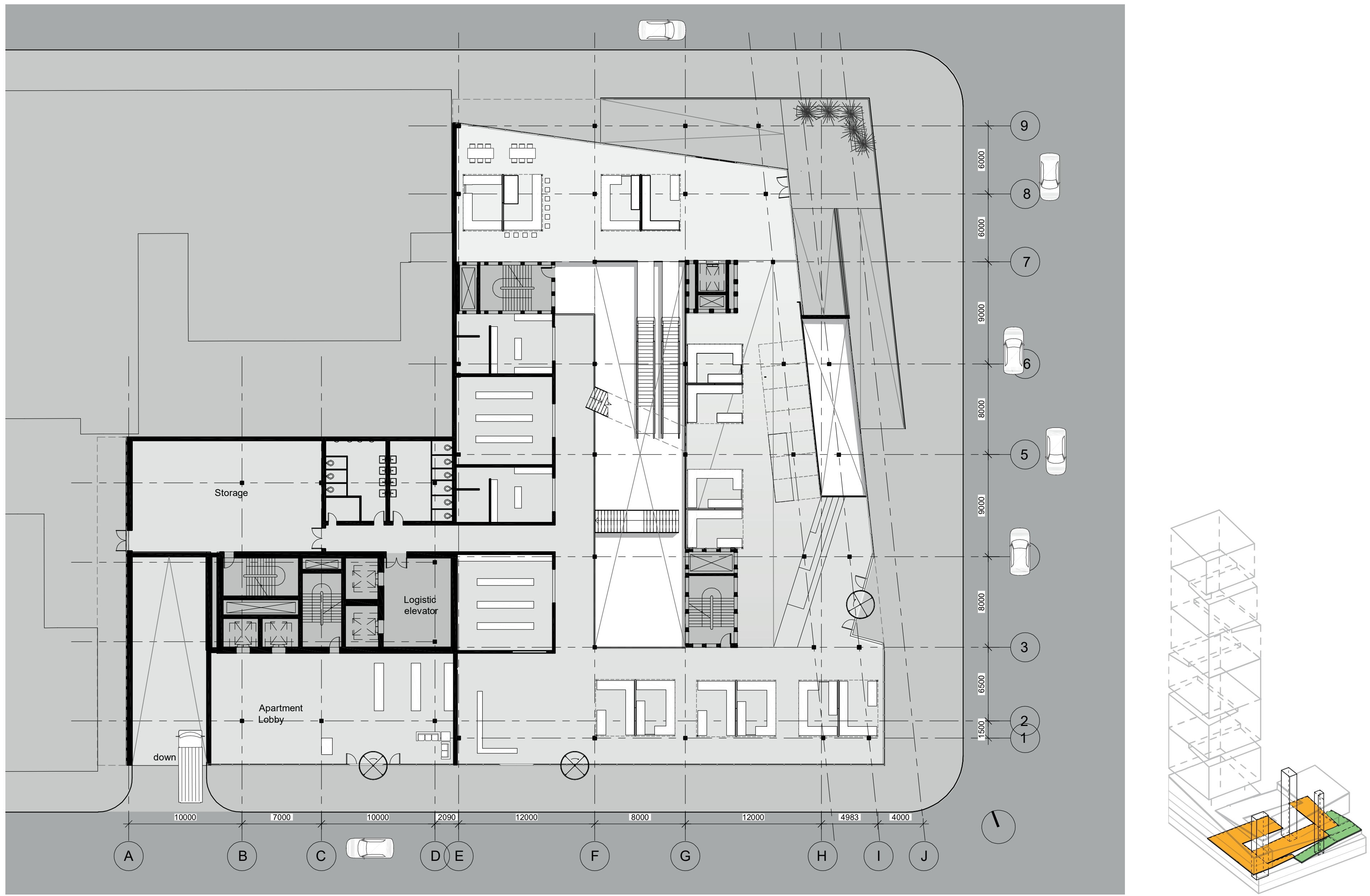

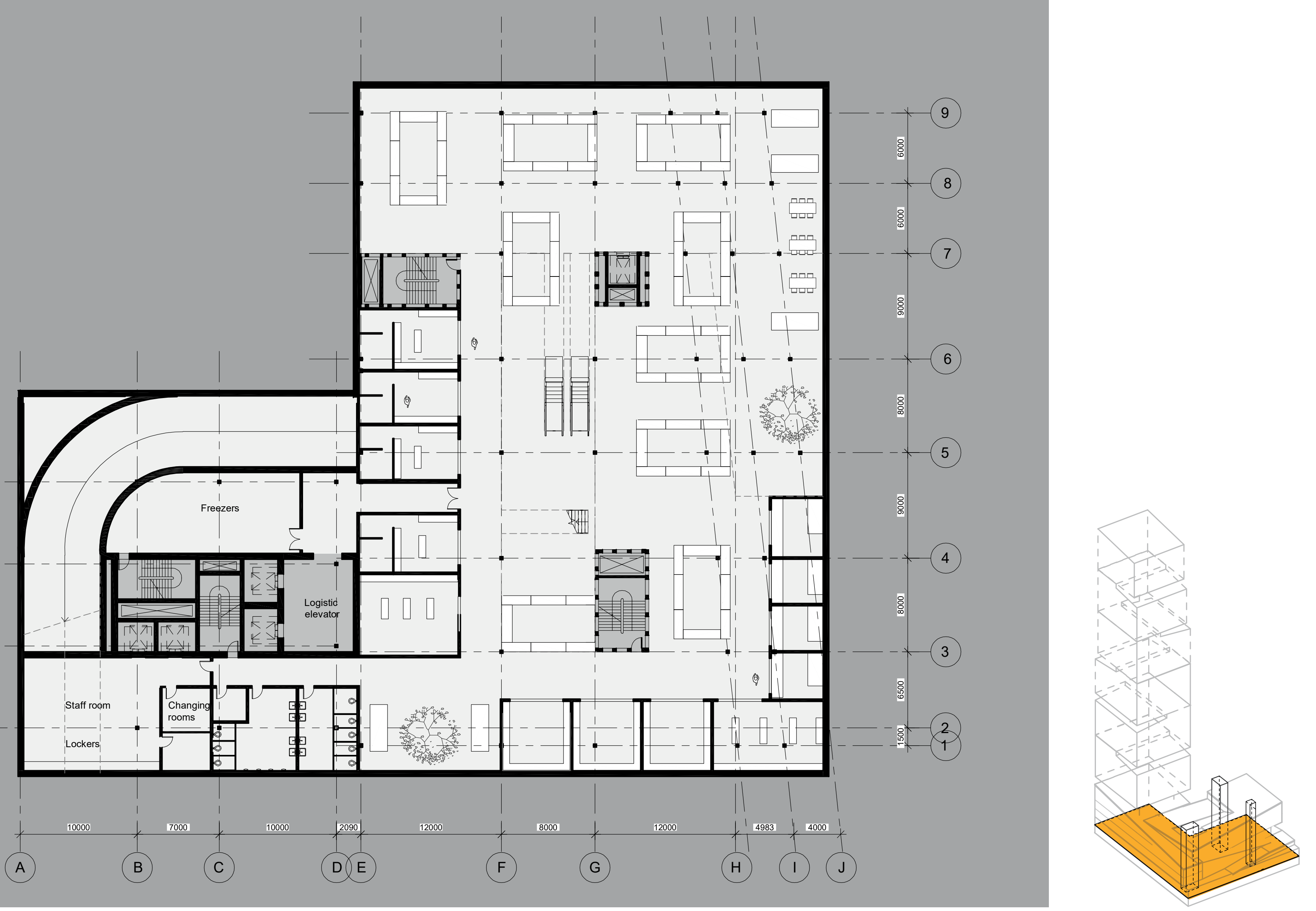


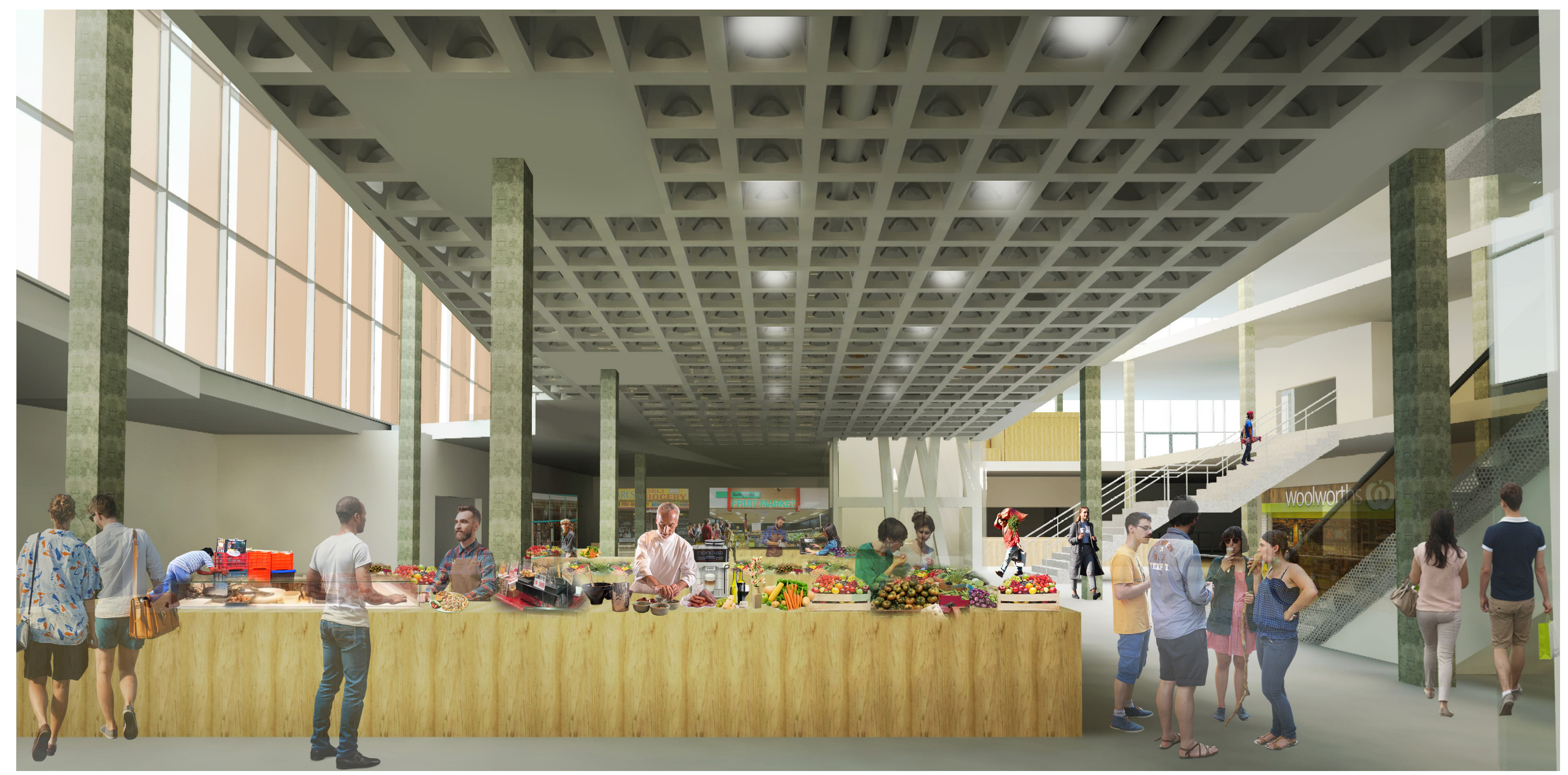



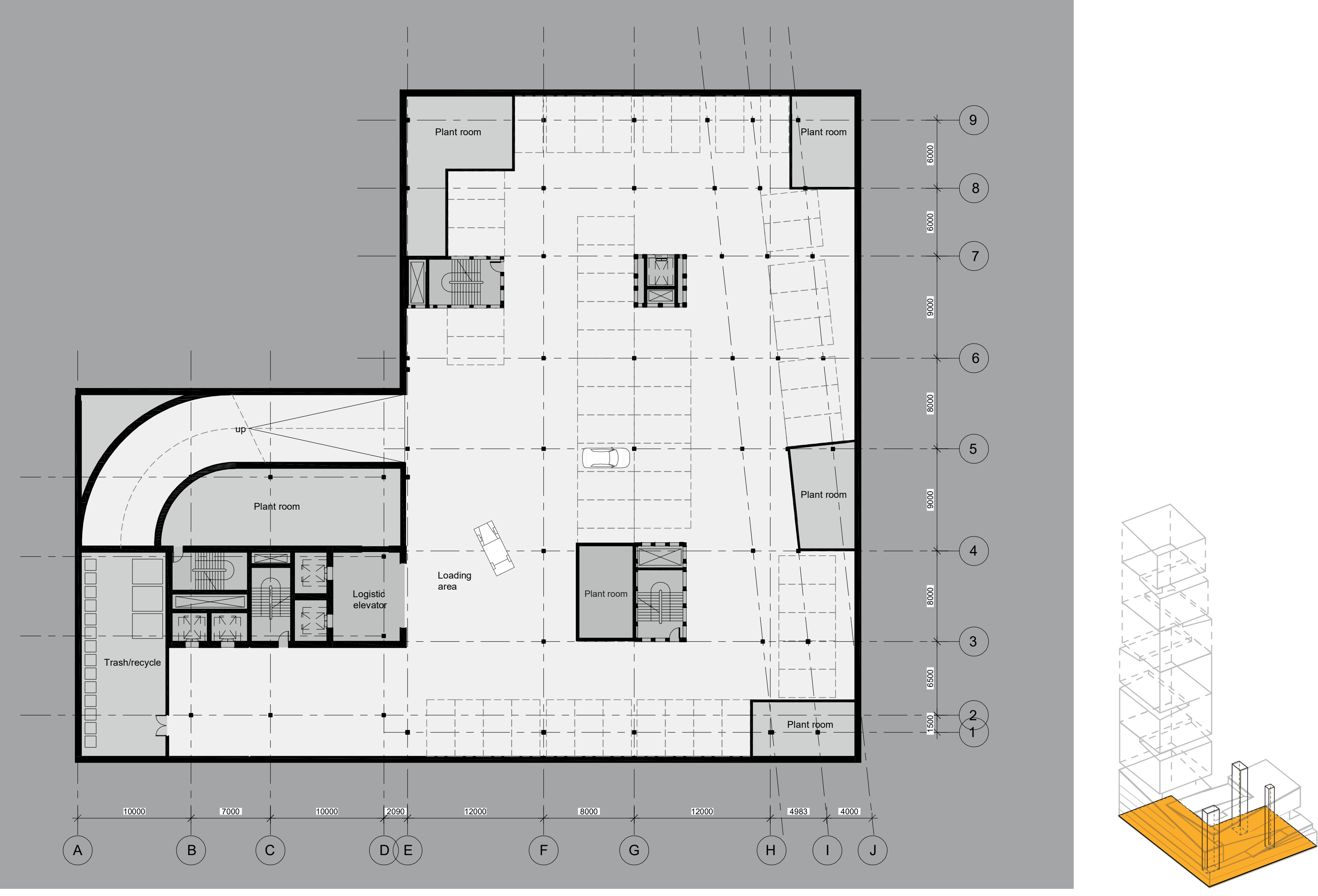


\section{Ground Floor}
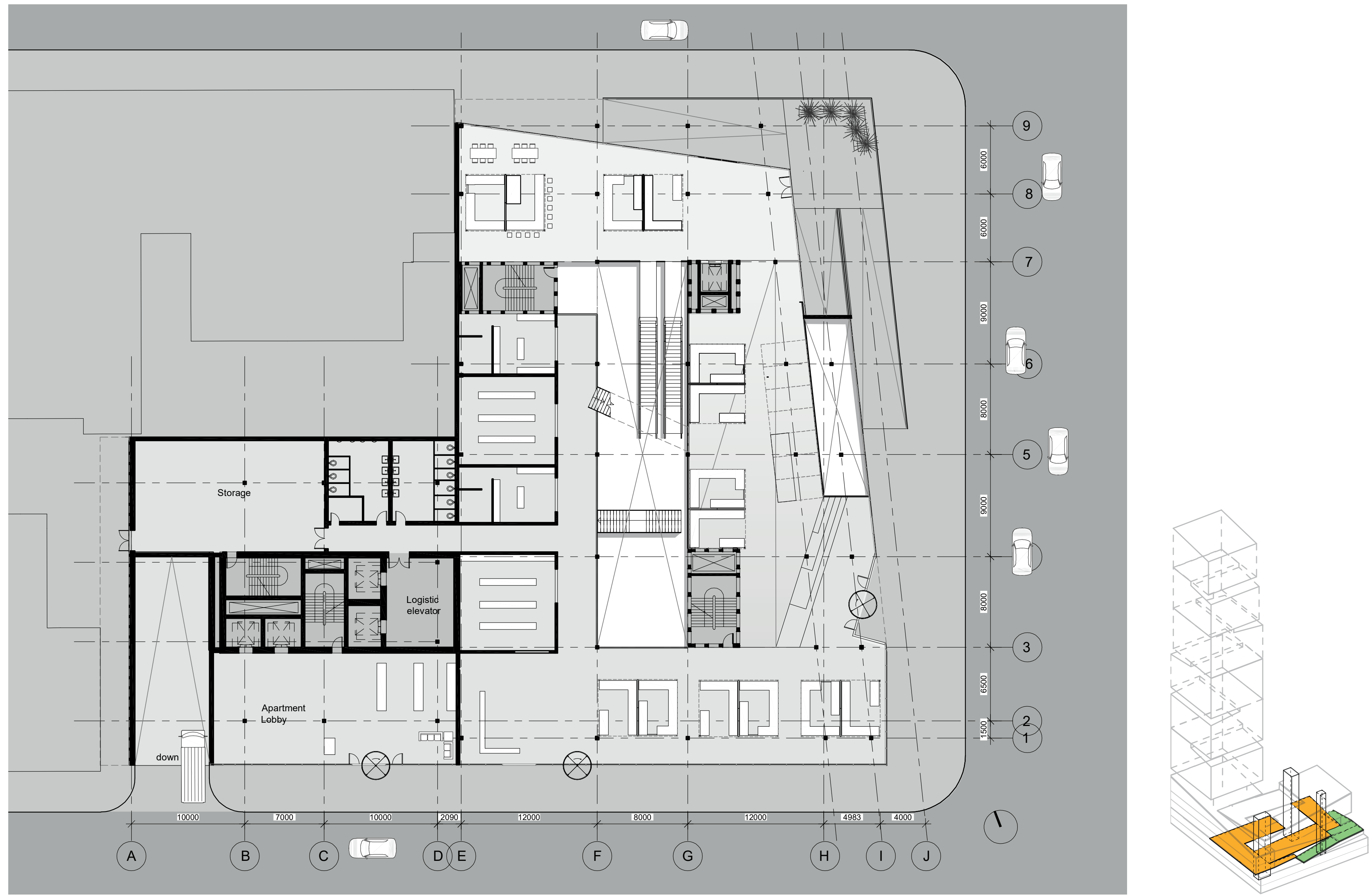

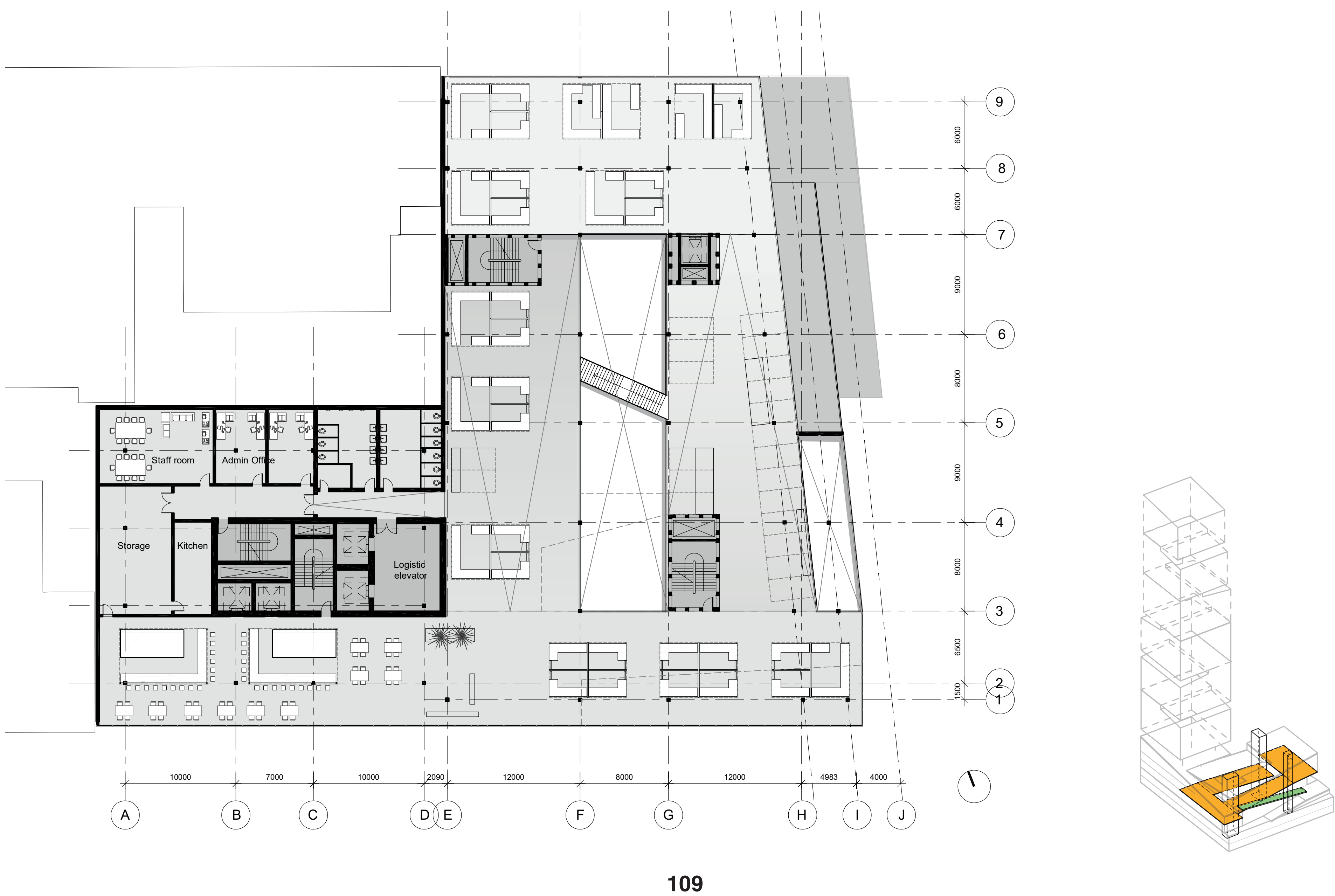


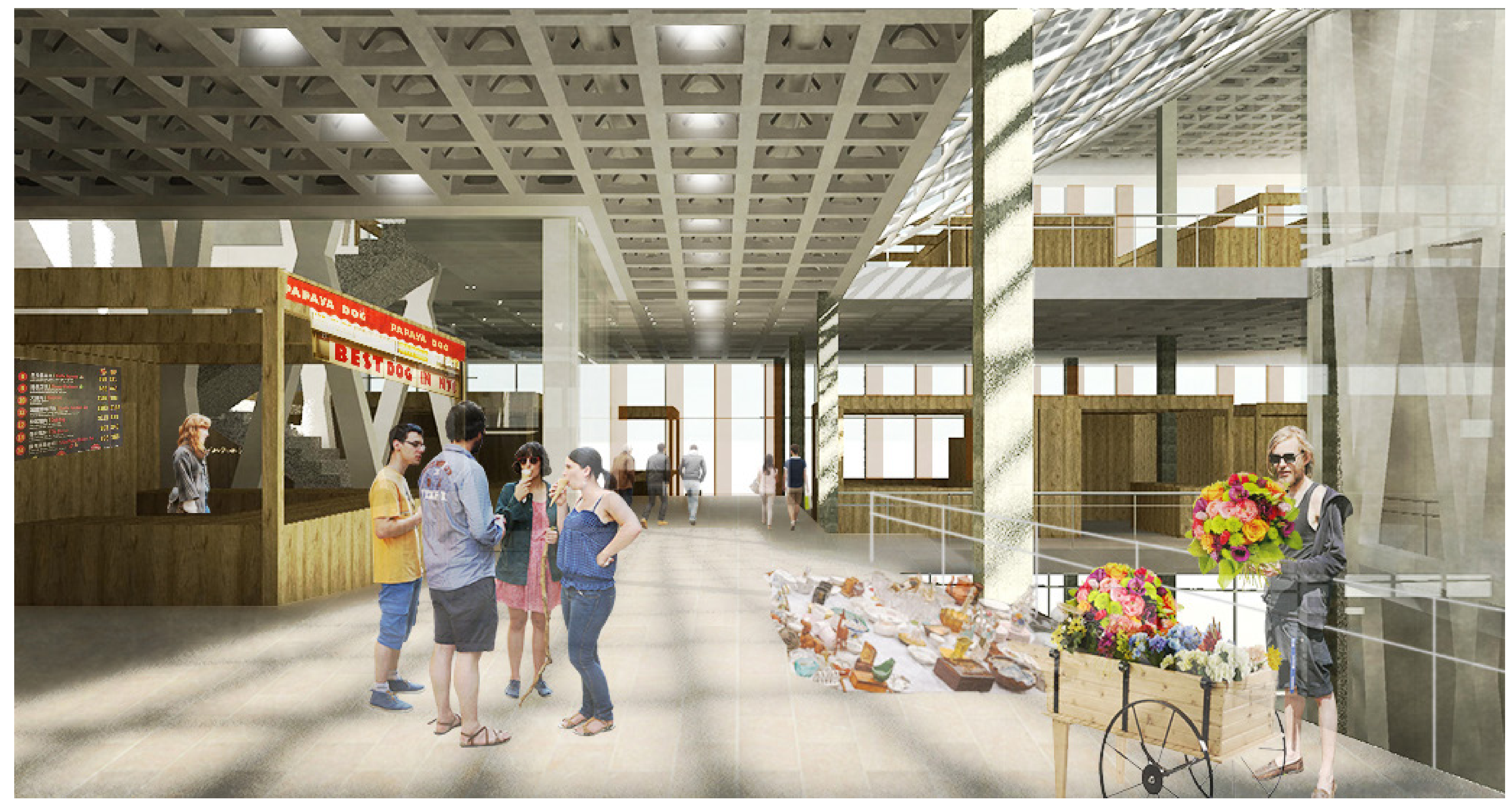




\section{Flexible stall system}
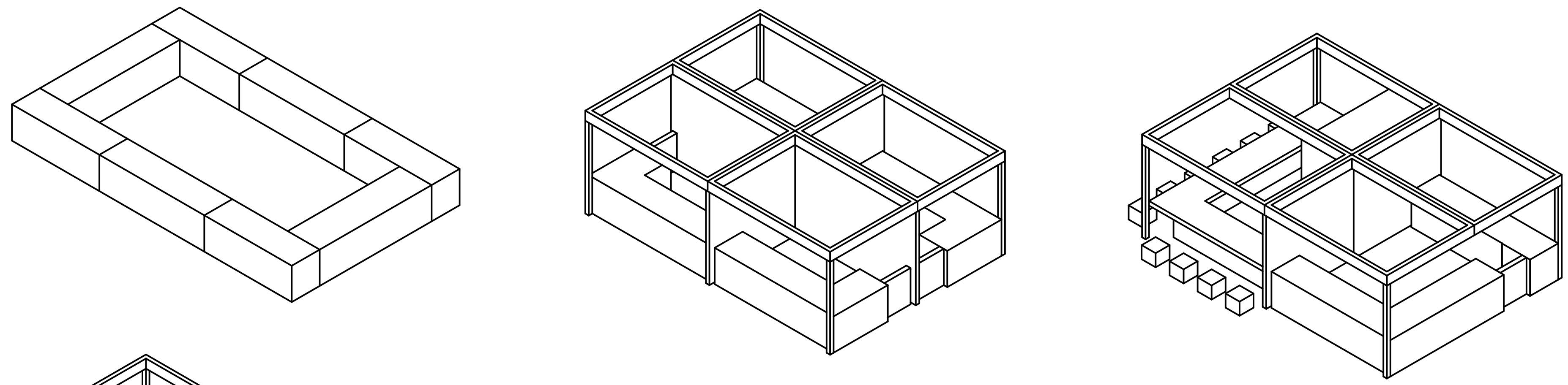

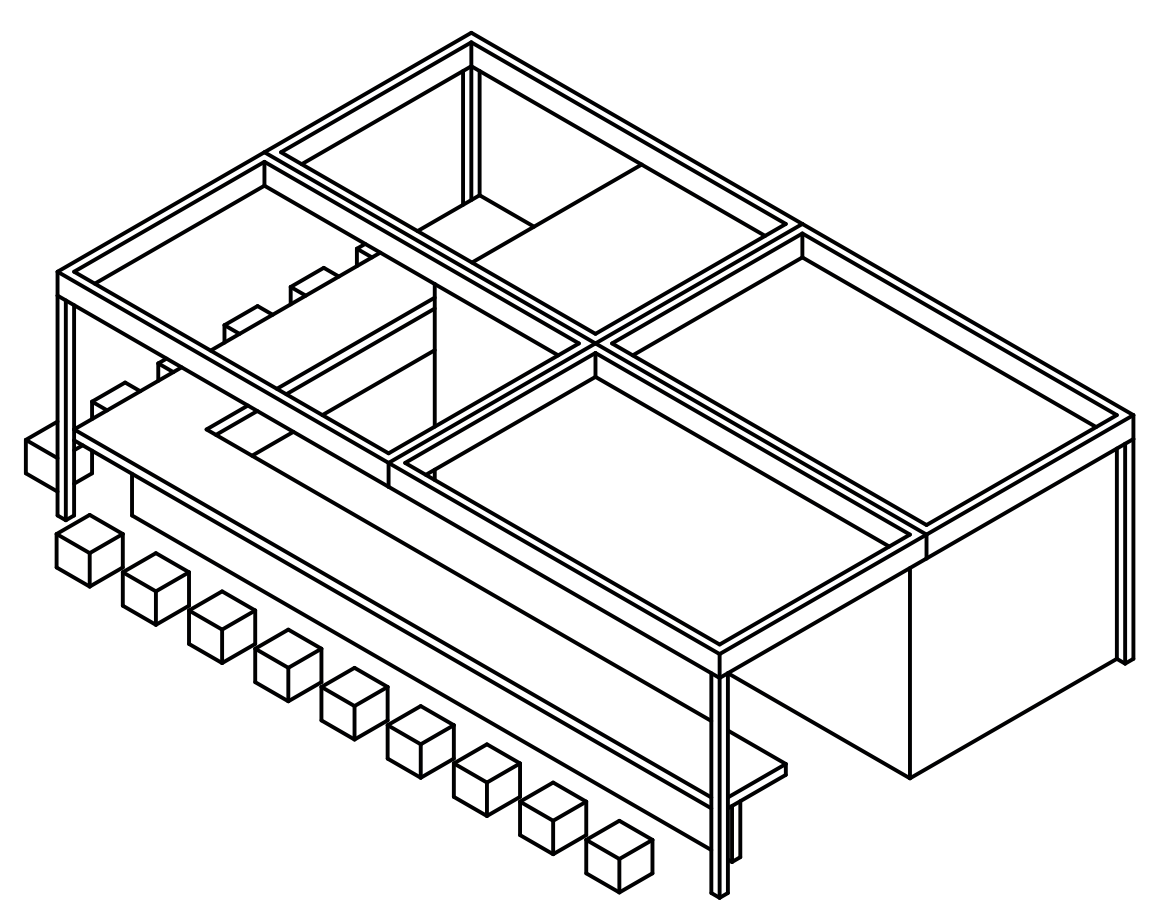

$4800 * 8000$

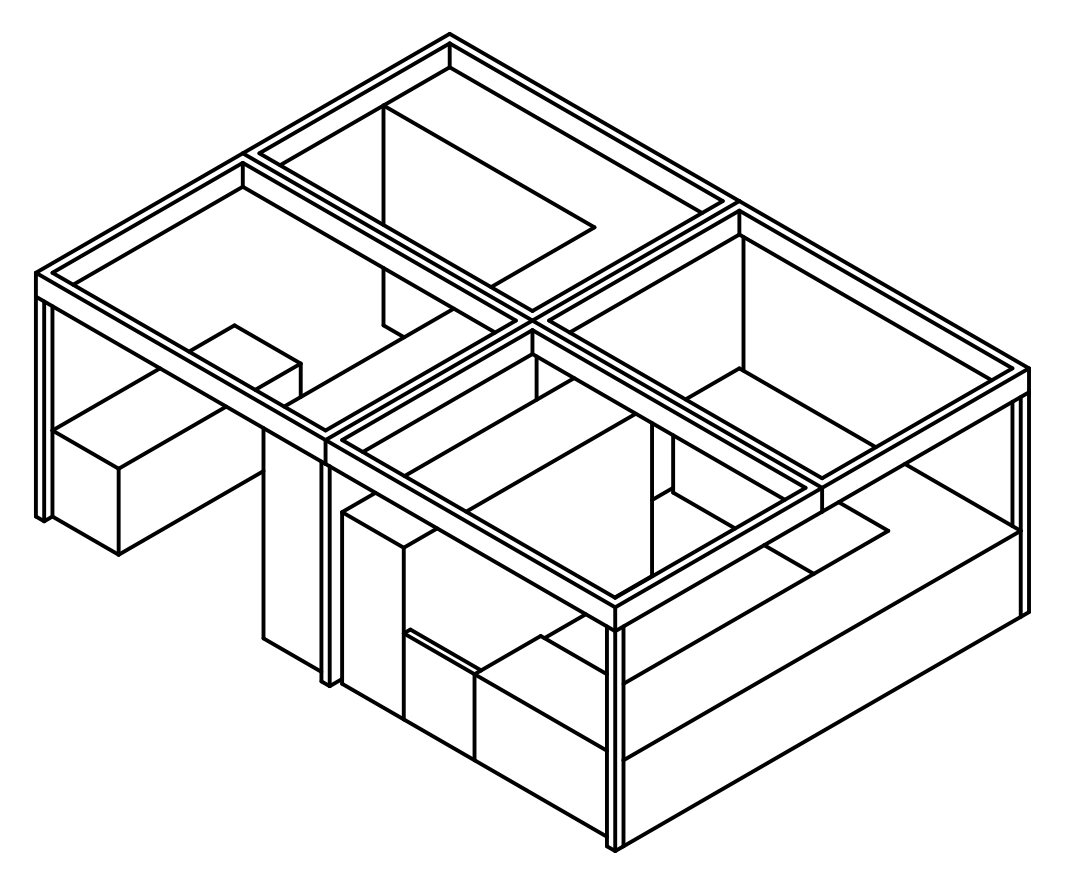

$5000 \star 7000$

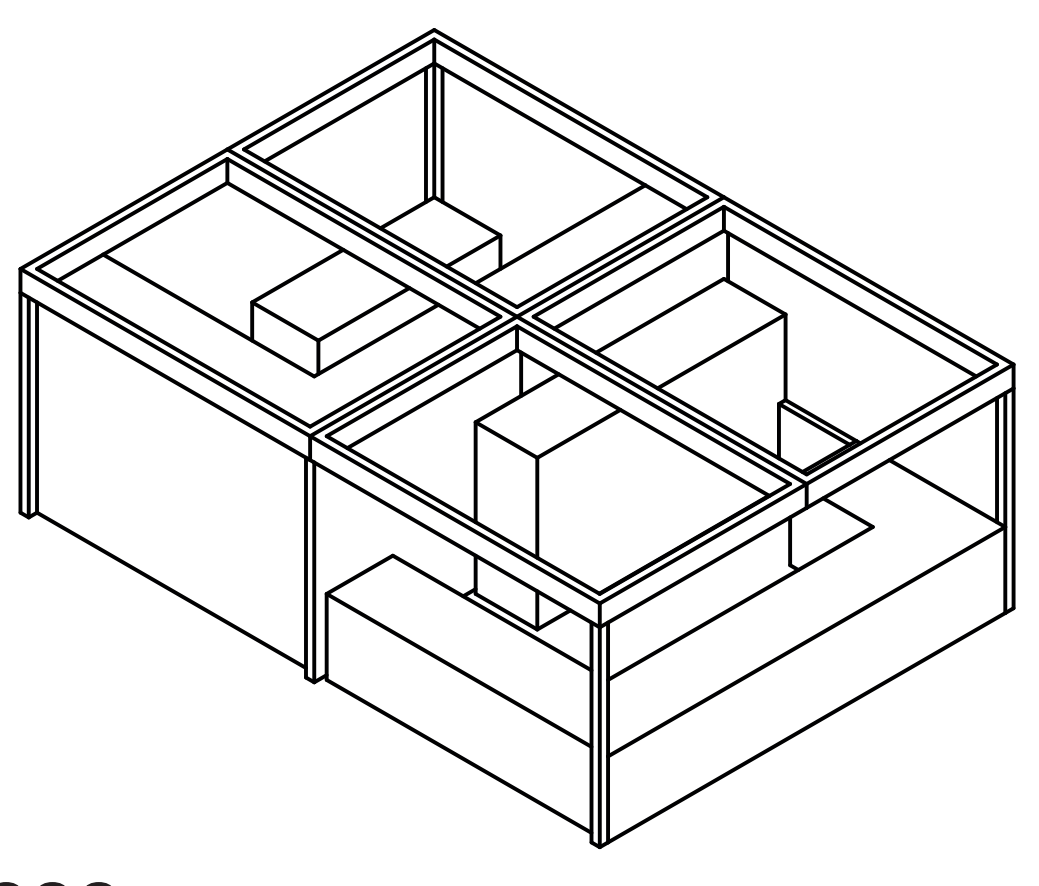




\section{2nd Floor}
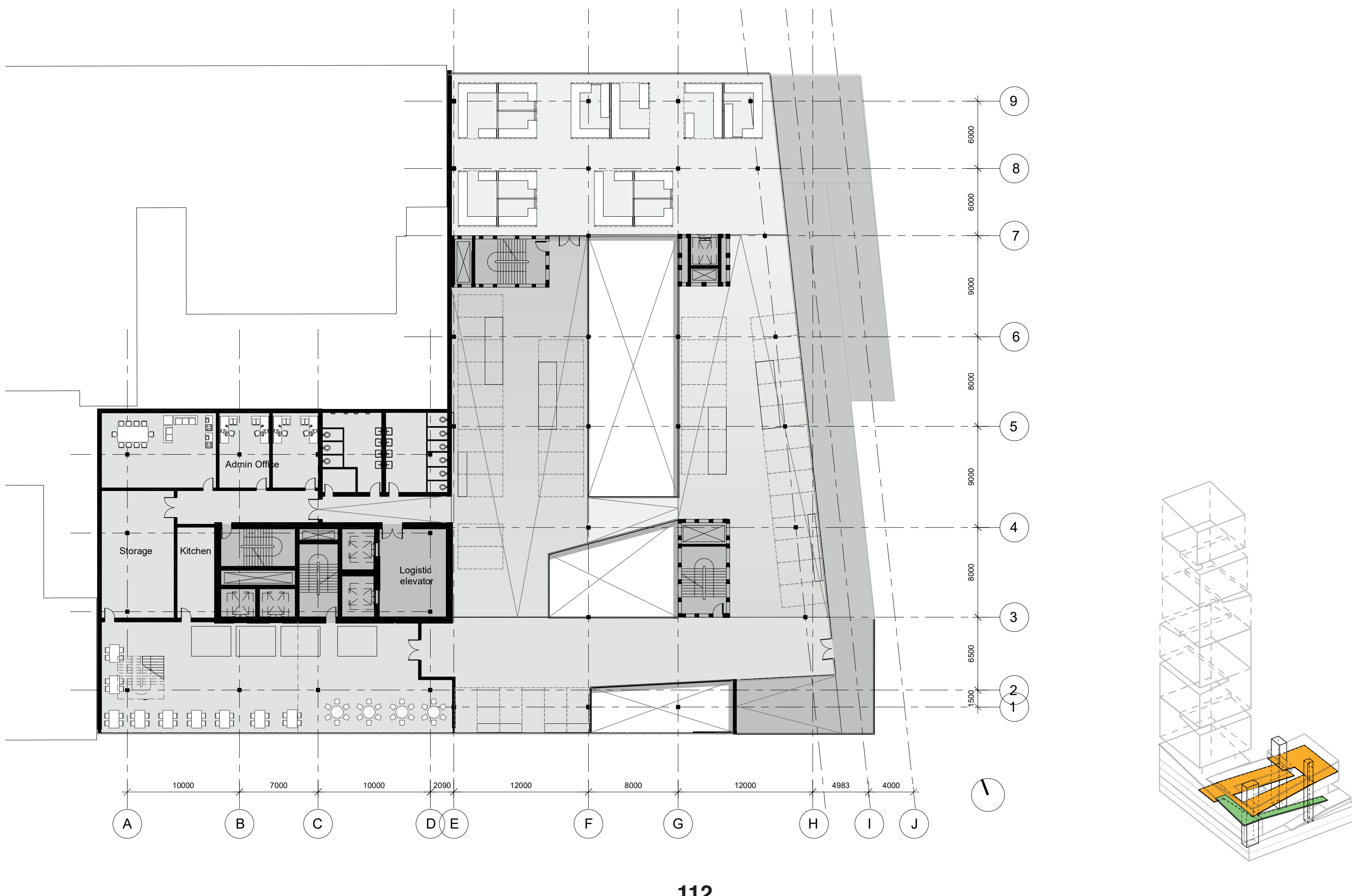

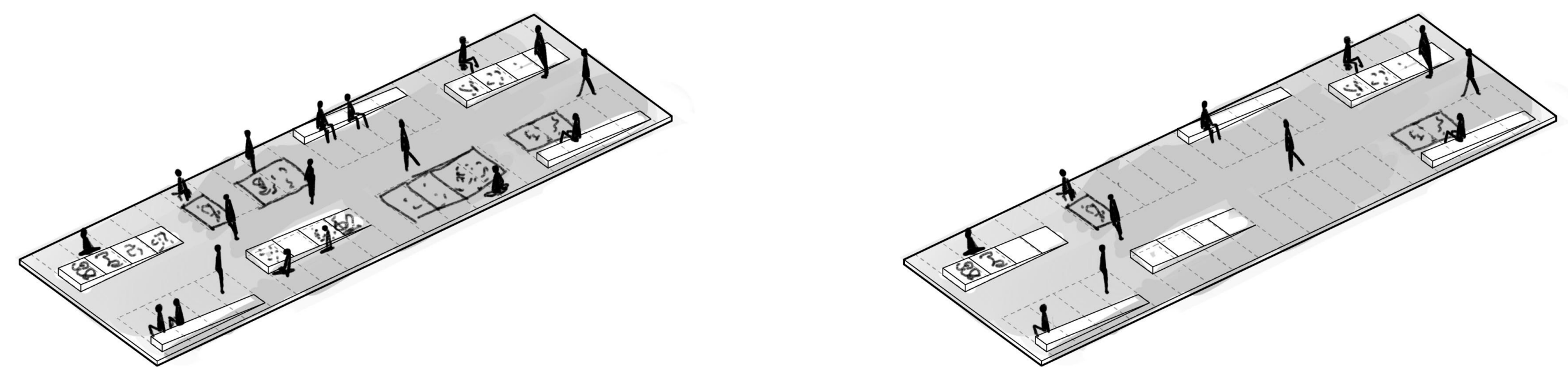


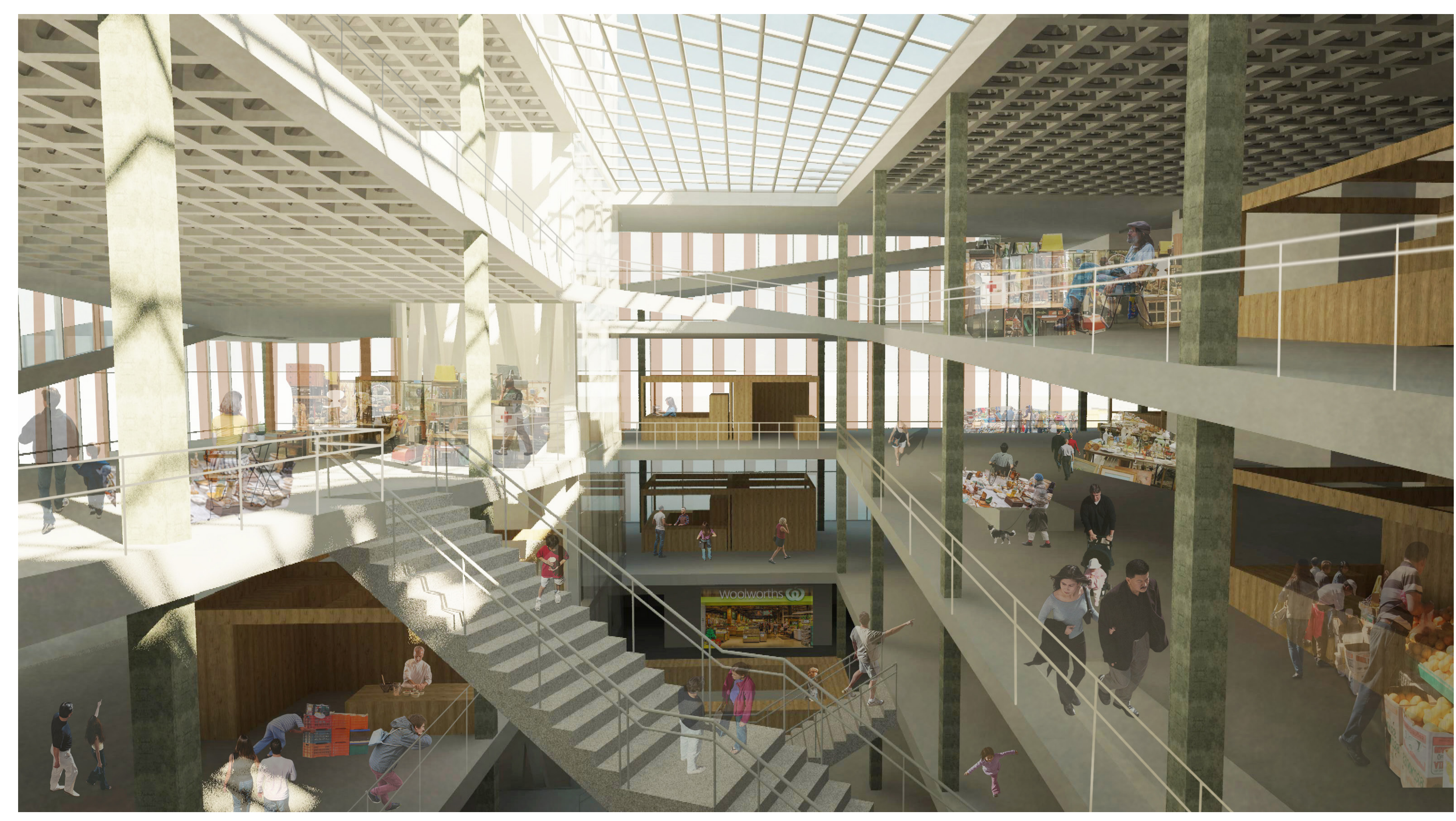




\section{Podium Rooftop}

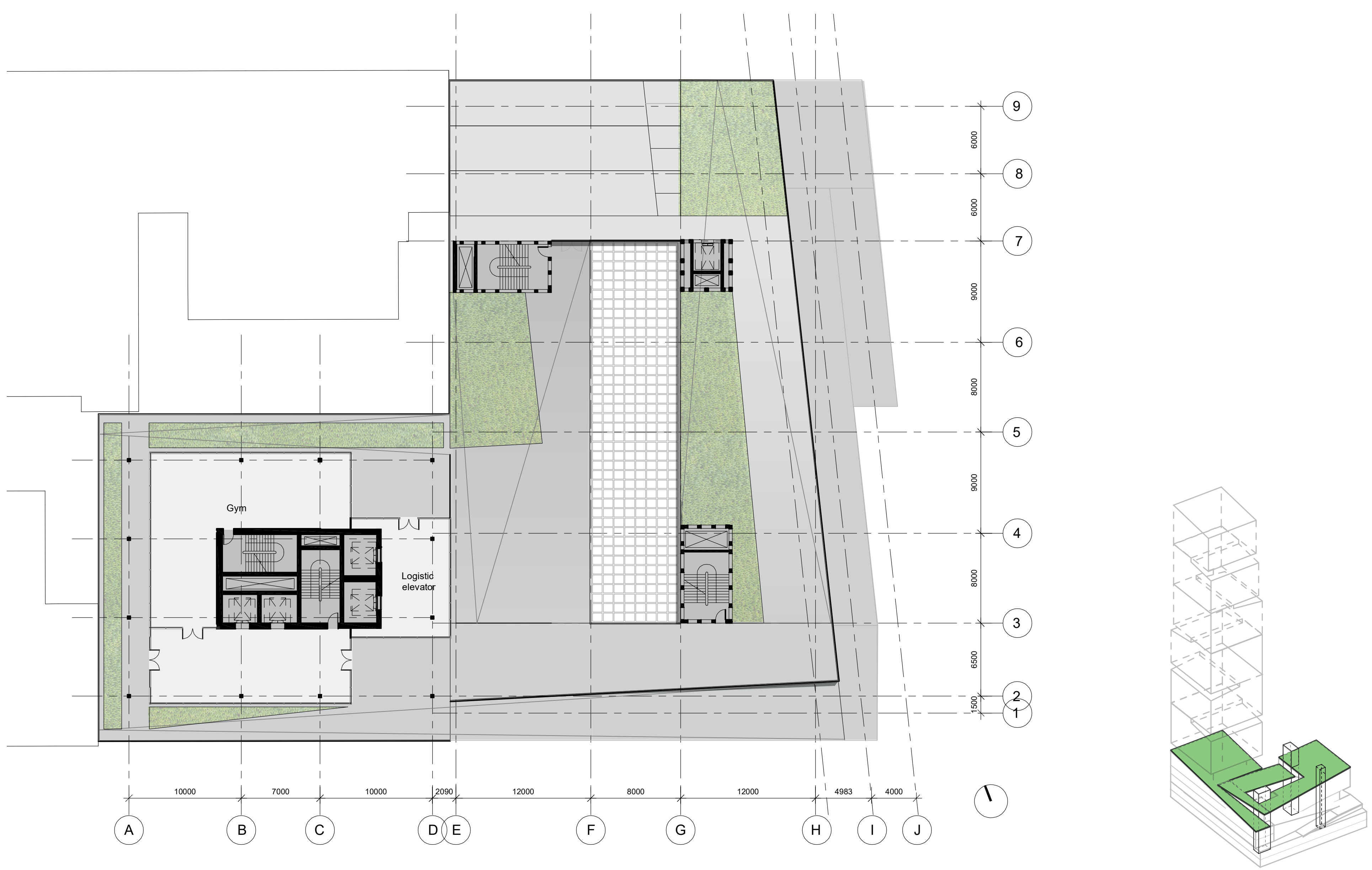




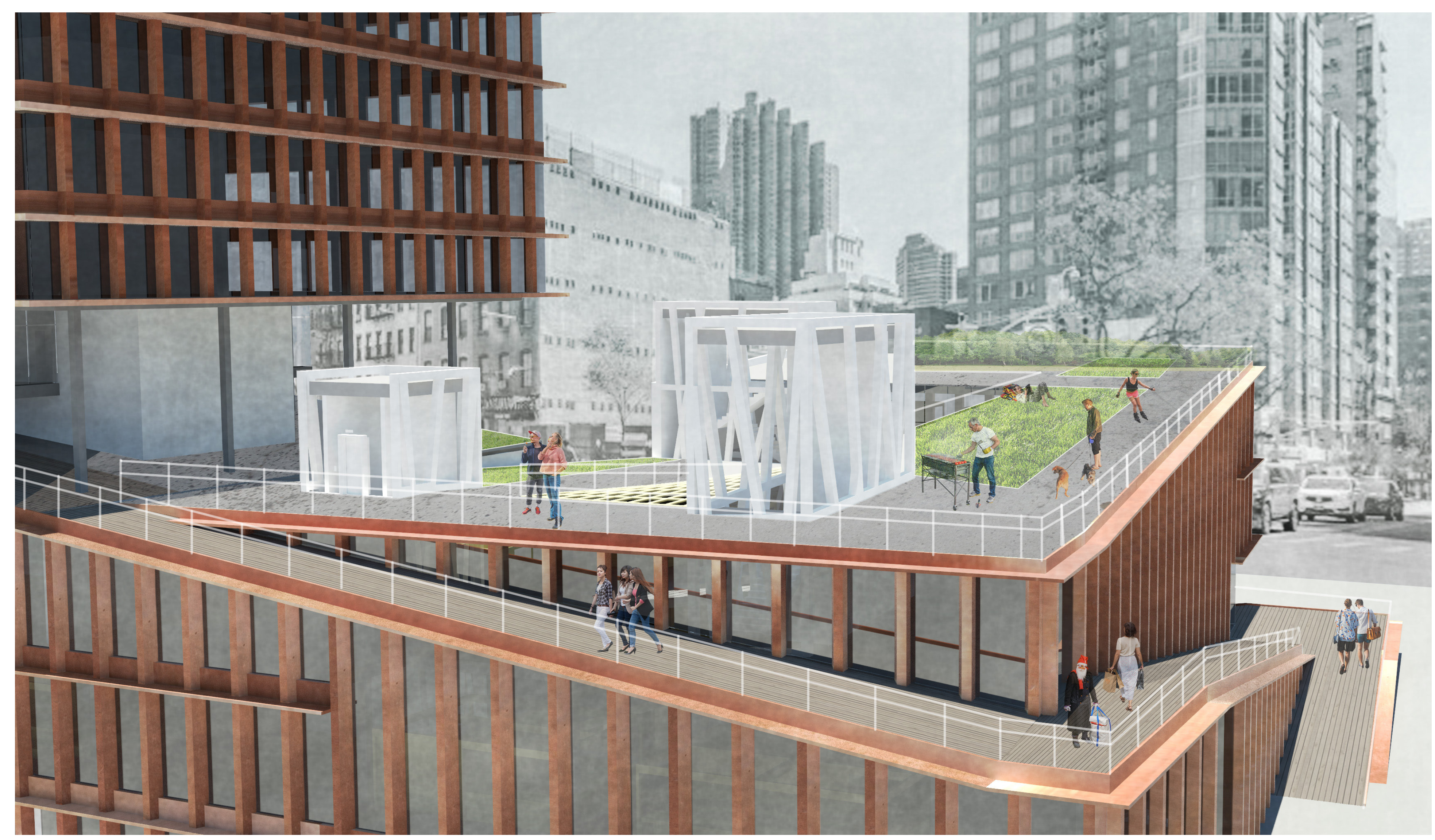




\section{Typical tower floor plan}
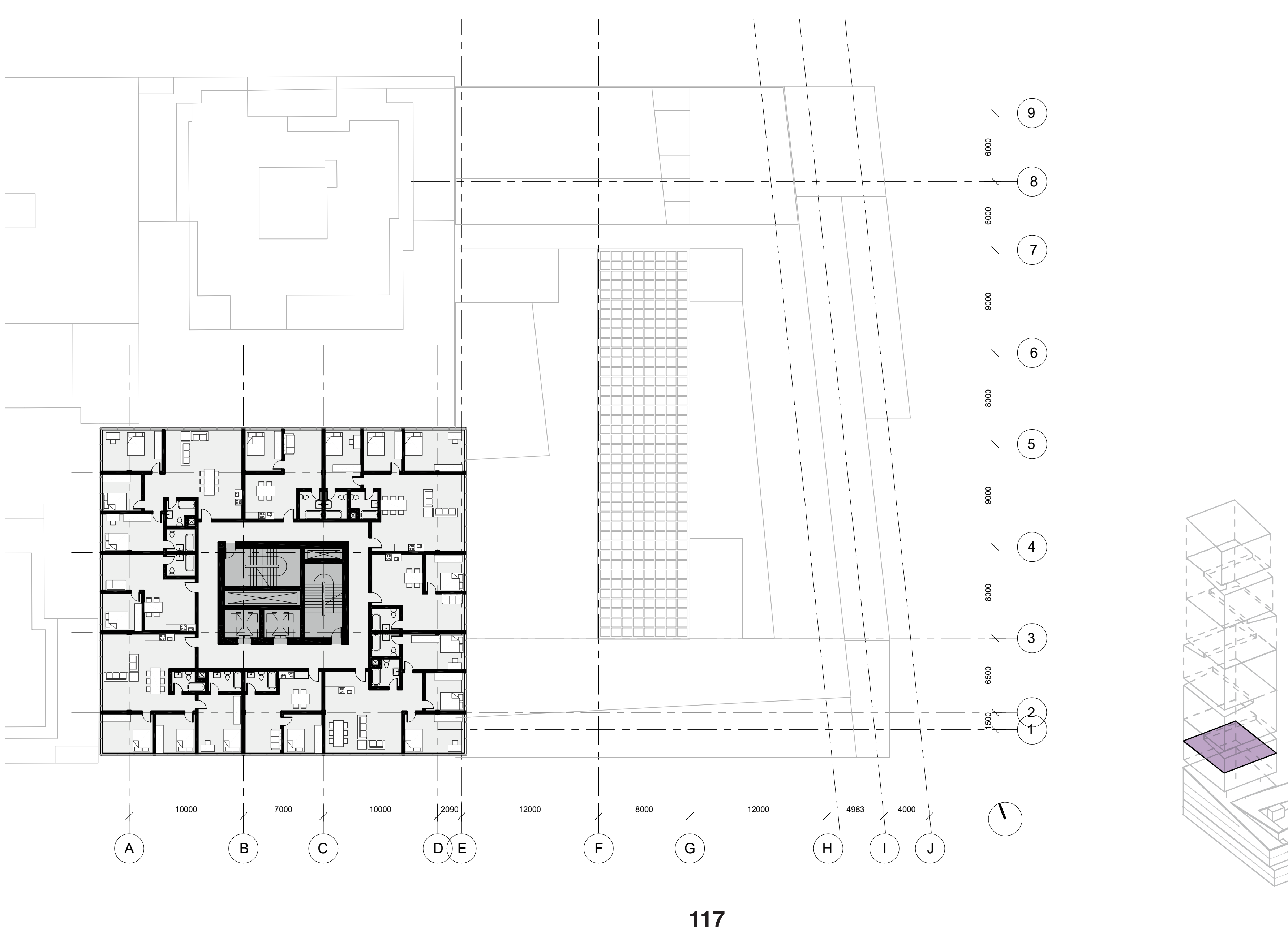


\section{Typical tower communal garden}

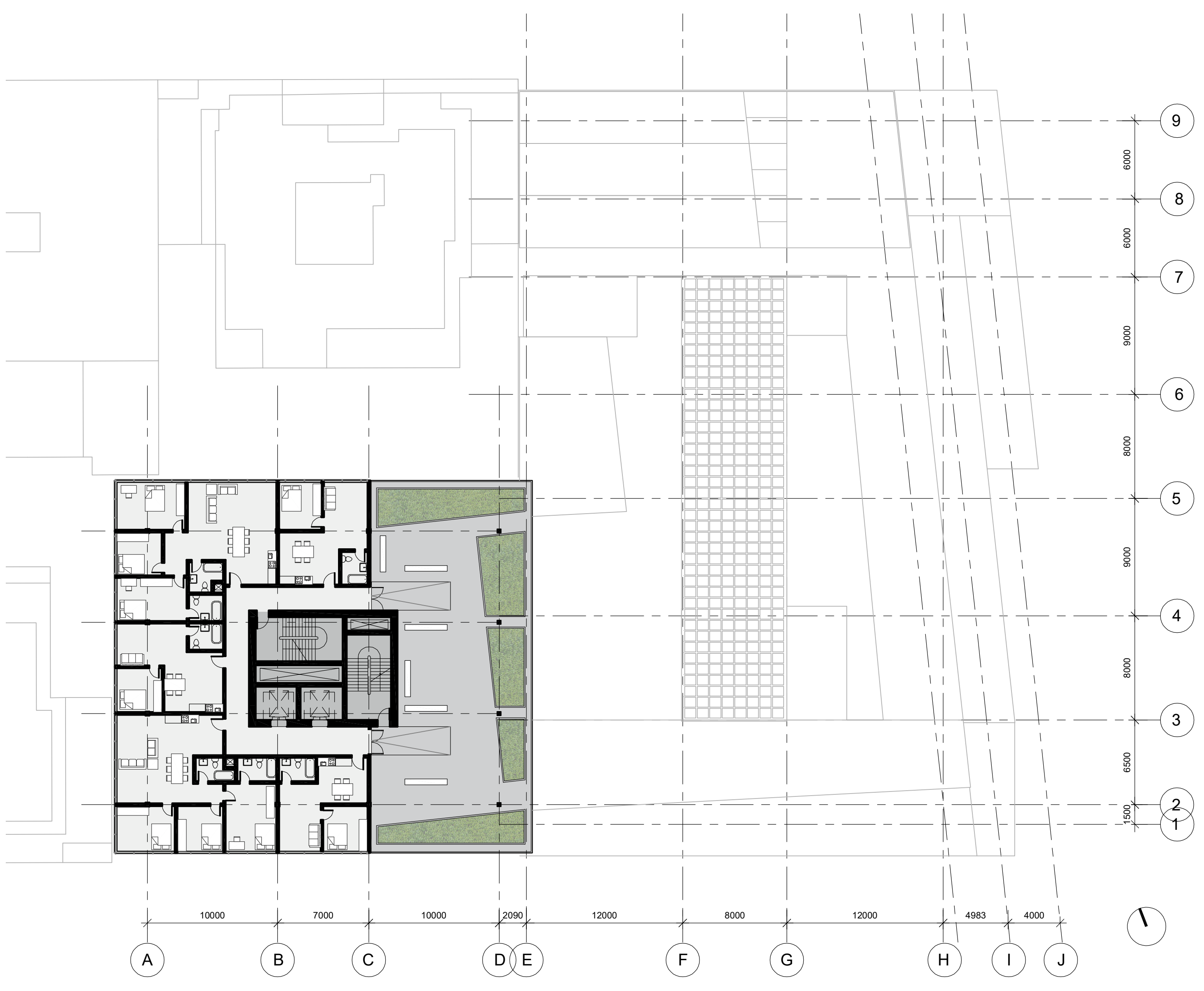


Vertical Transportation

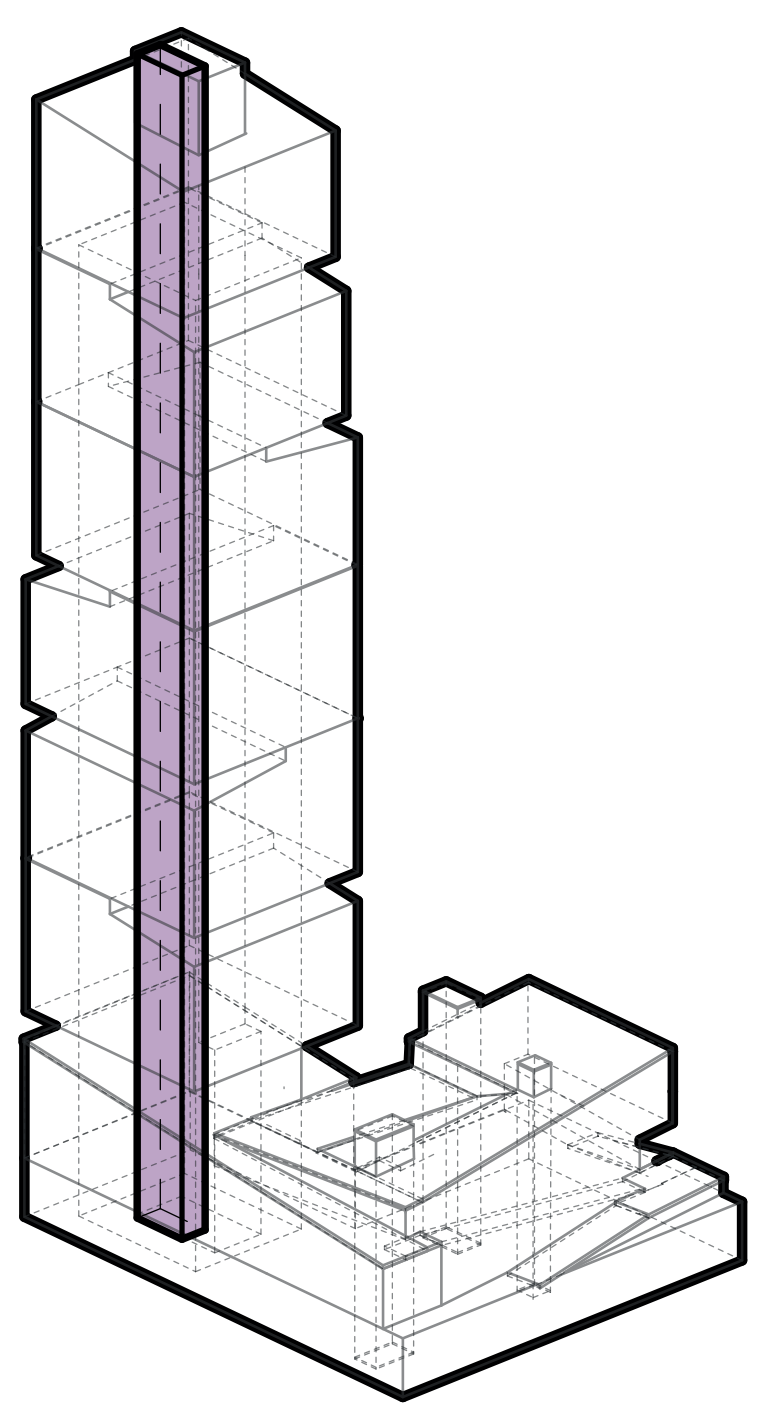

elevators for residents

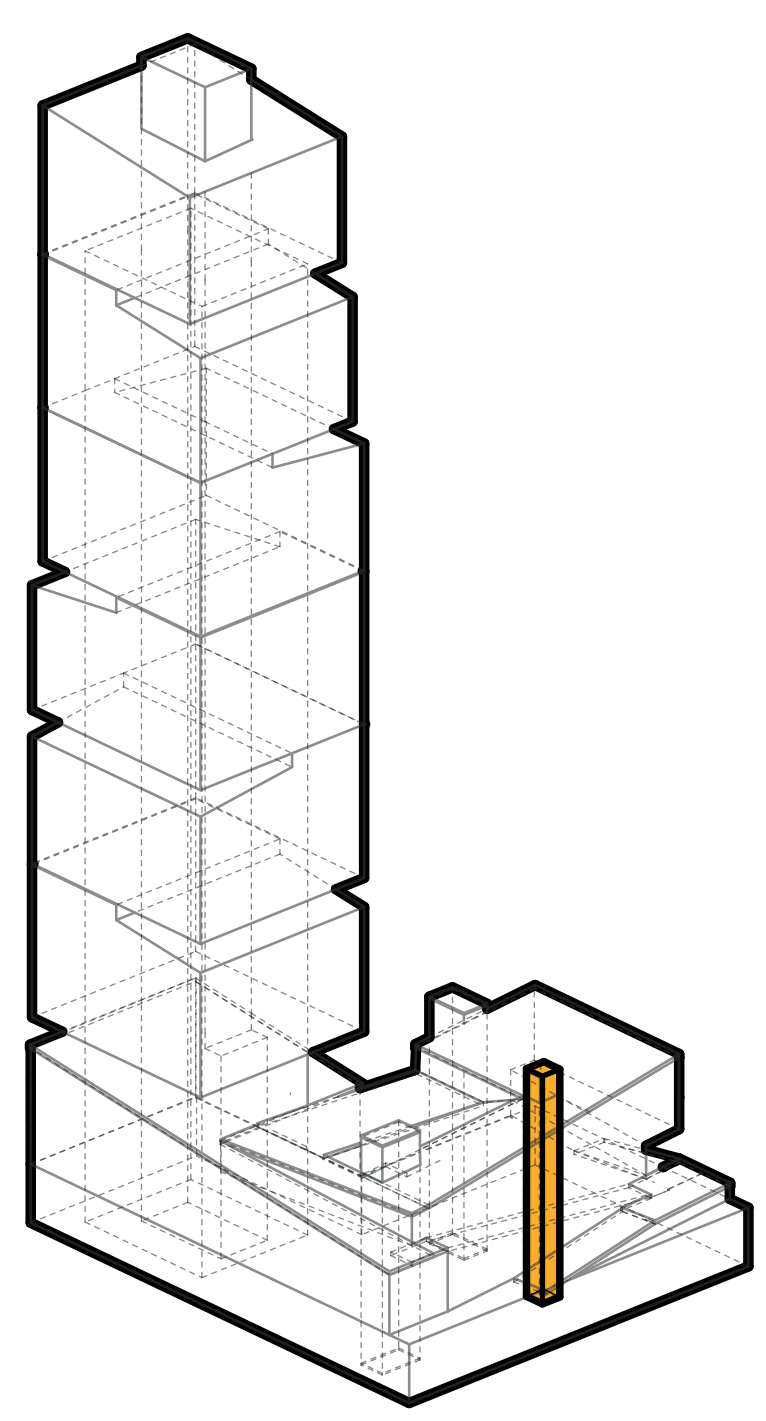

elevators for market customer

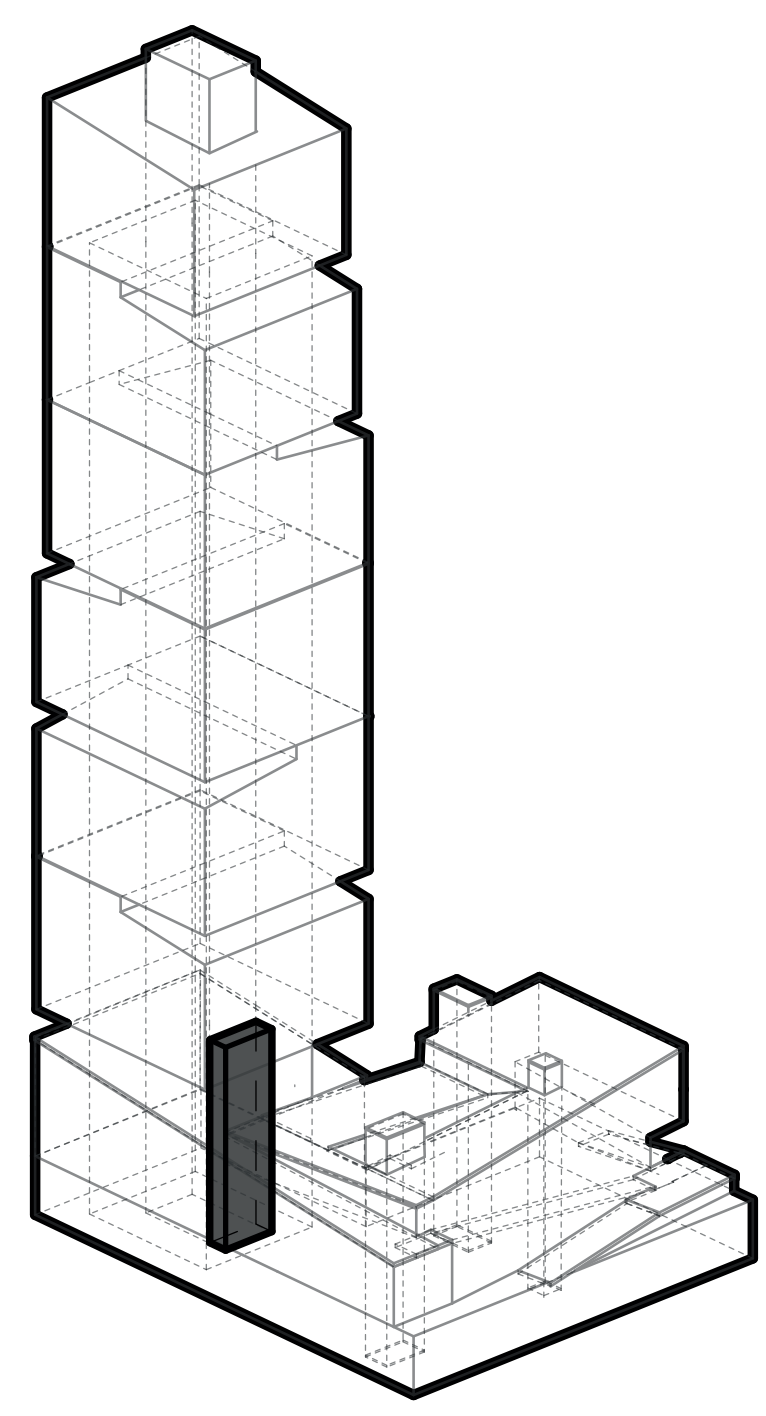

freight elevators and storage

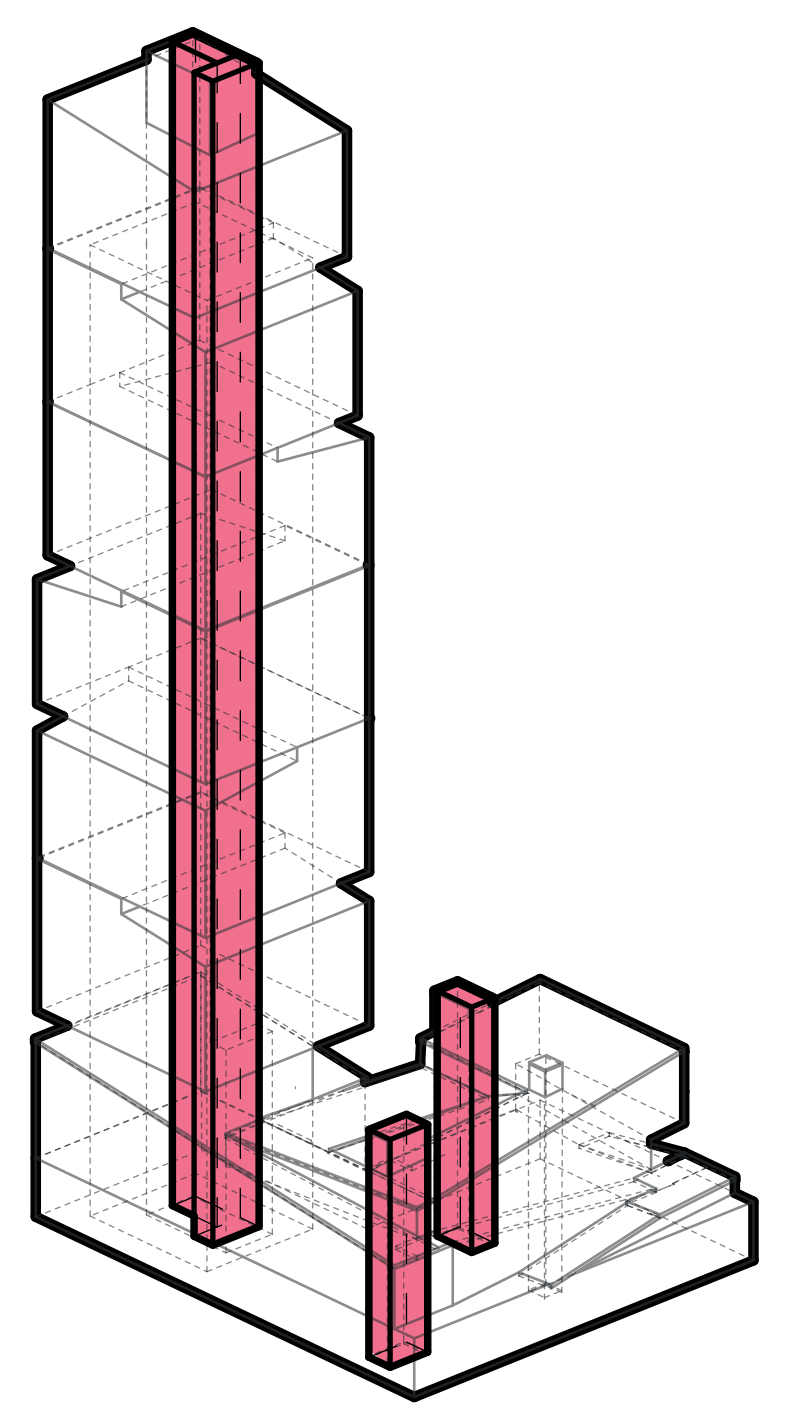

fire escape stairs

119 


\section{East Elevation}
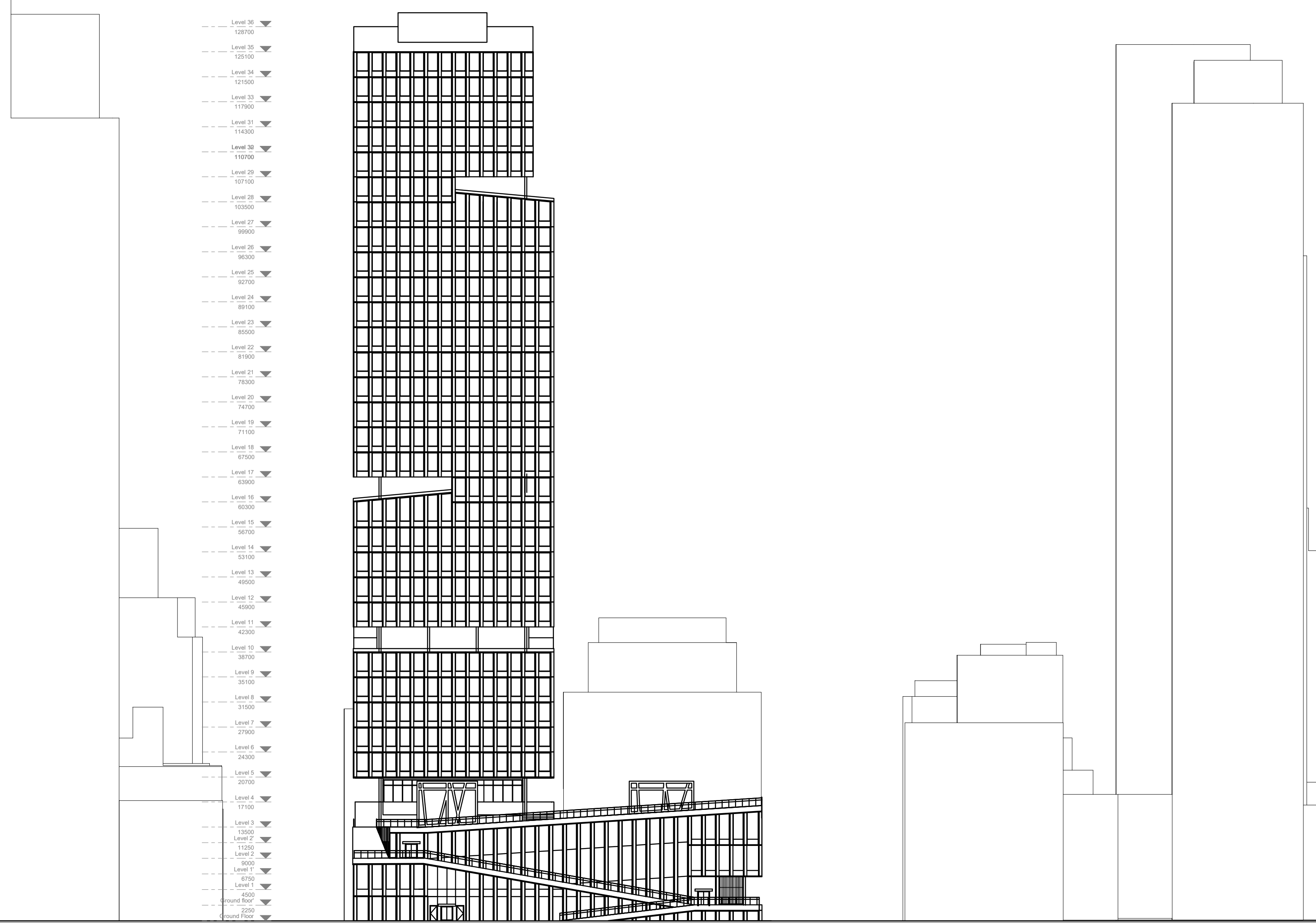

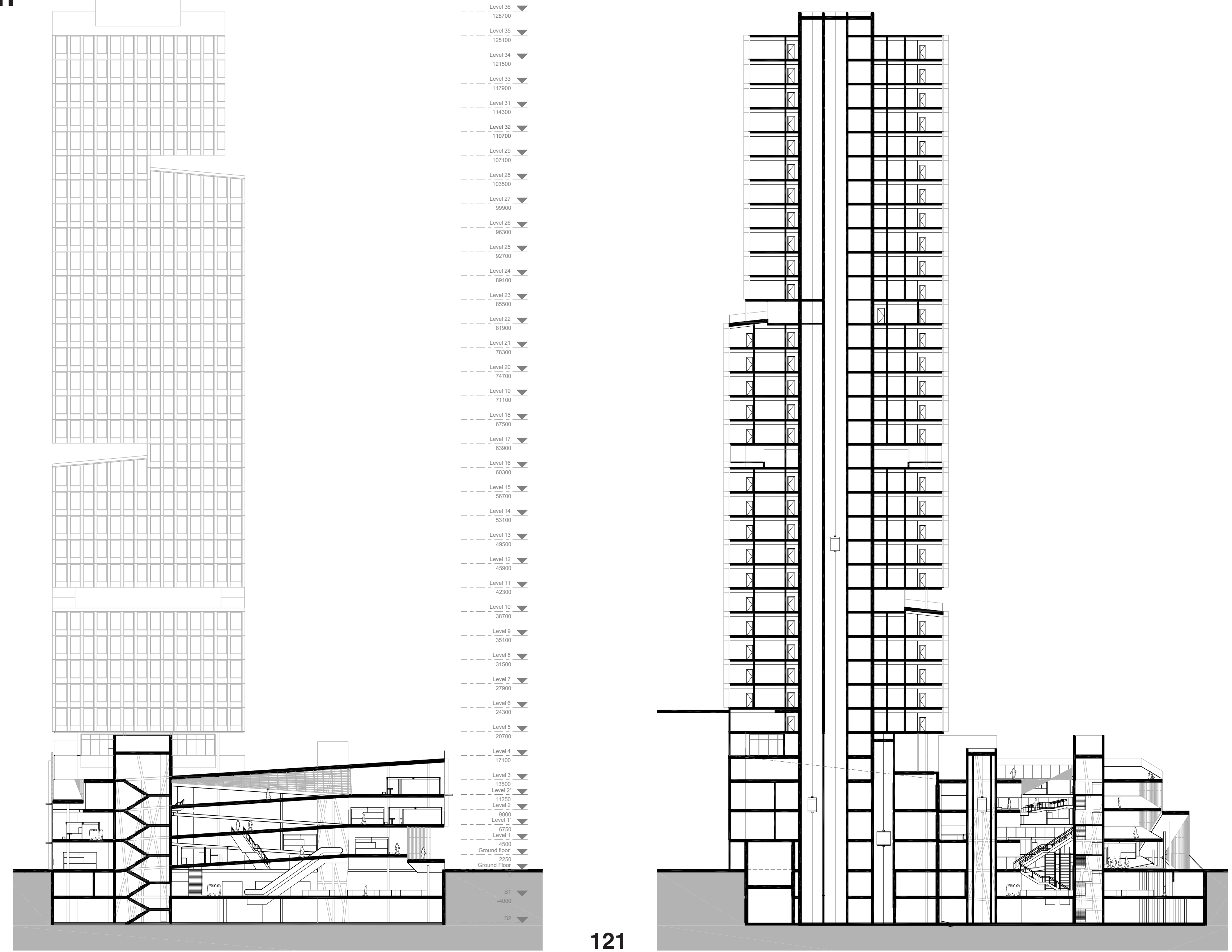


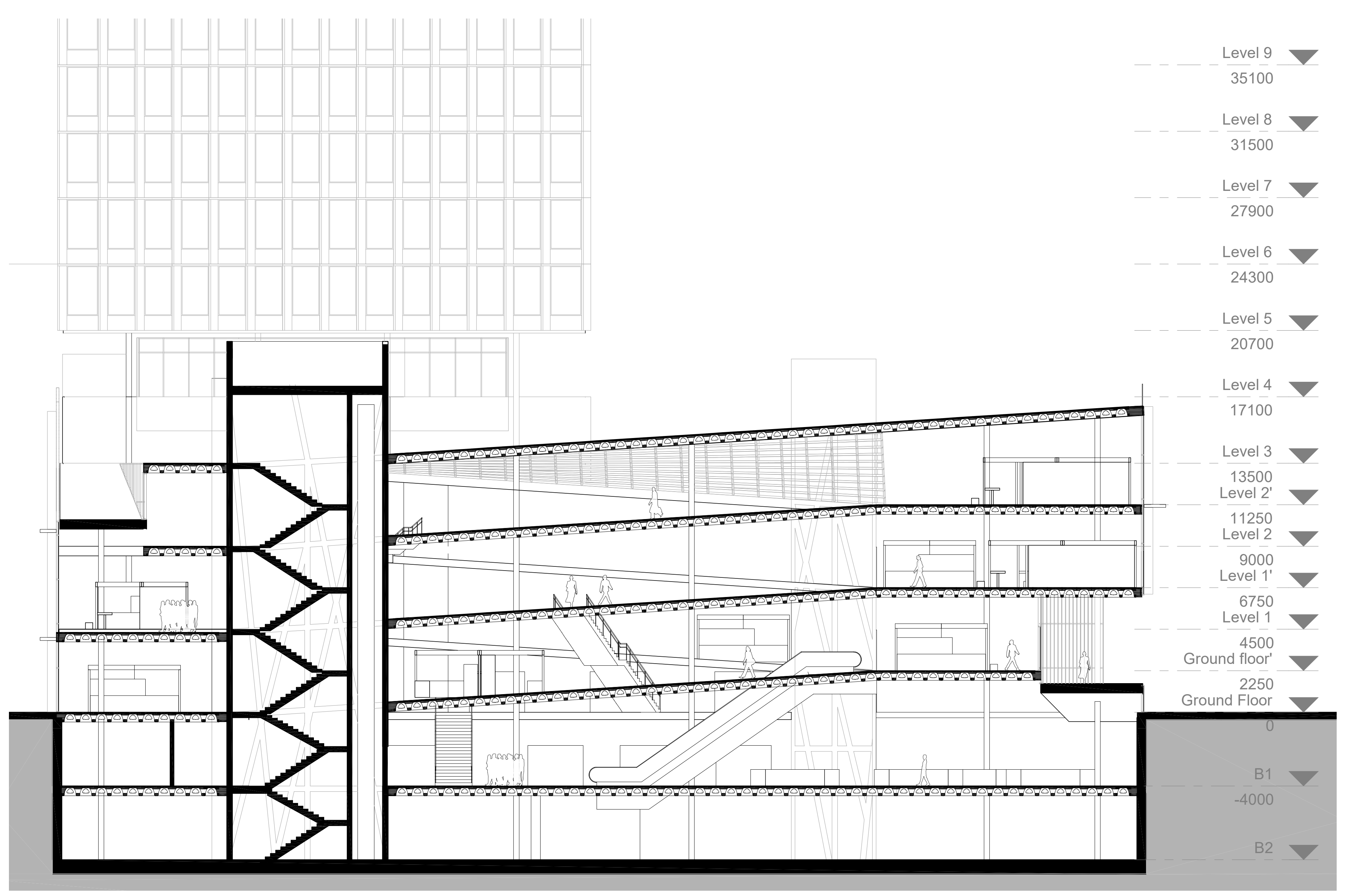




\section{Materialization}

Copper
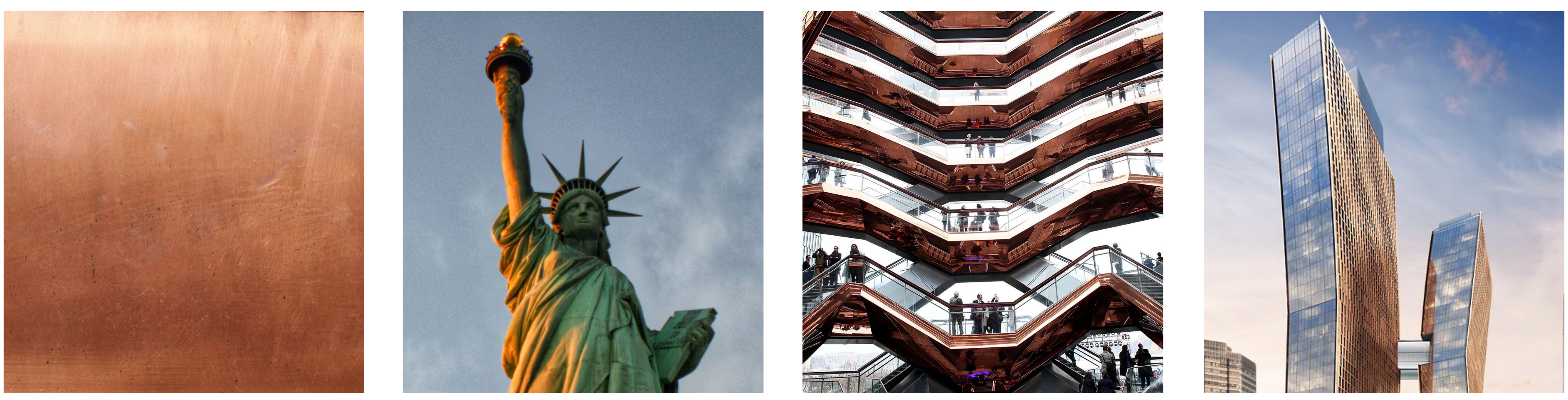
Materialization

Copper

Highly recycled content

long life span

corrosion resistant

low maintenance cost 


\section{Materialization}

Copper
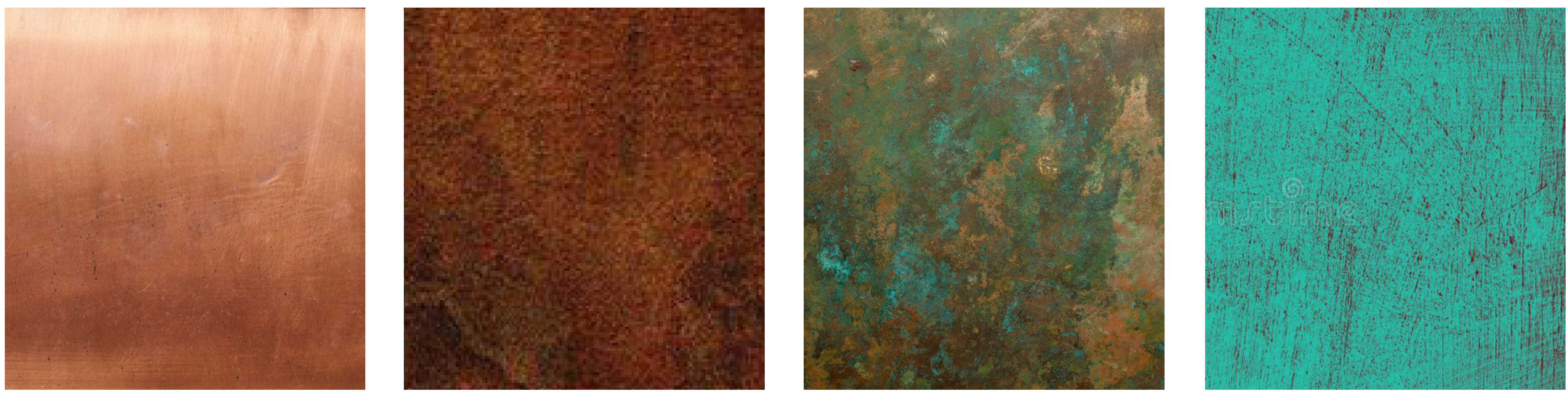
Materialization
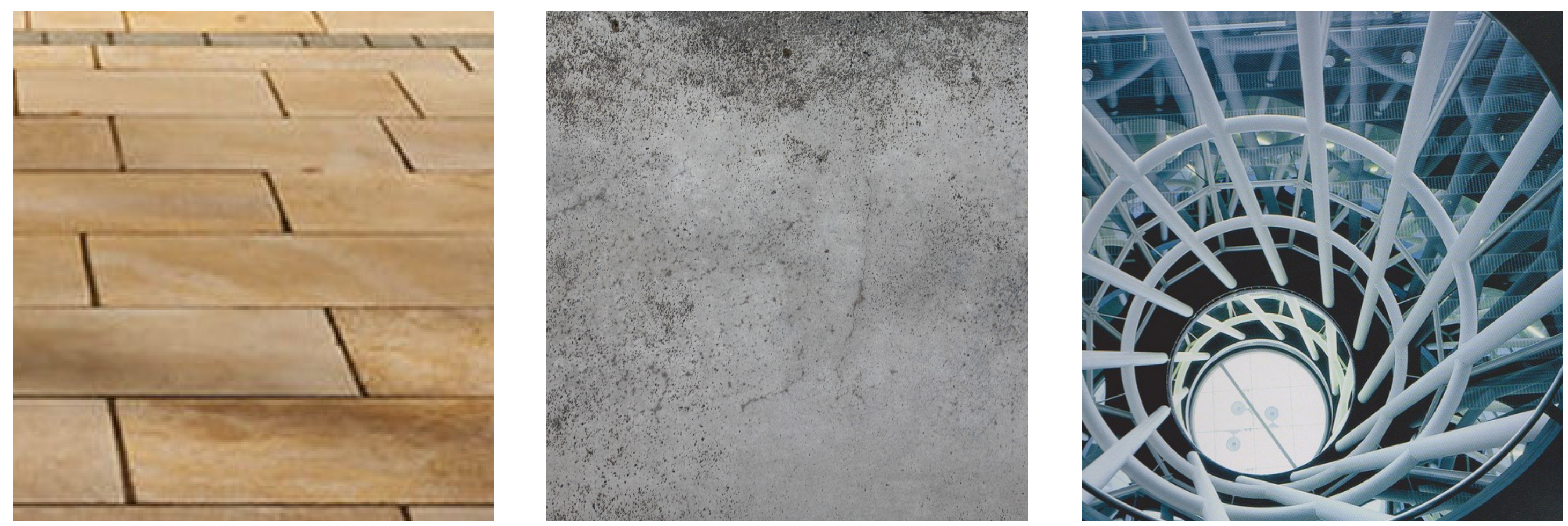


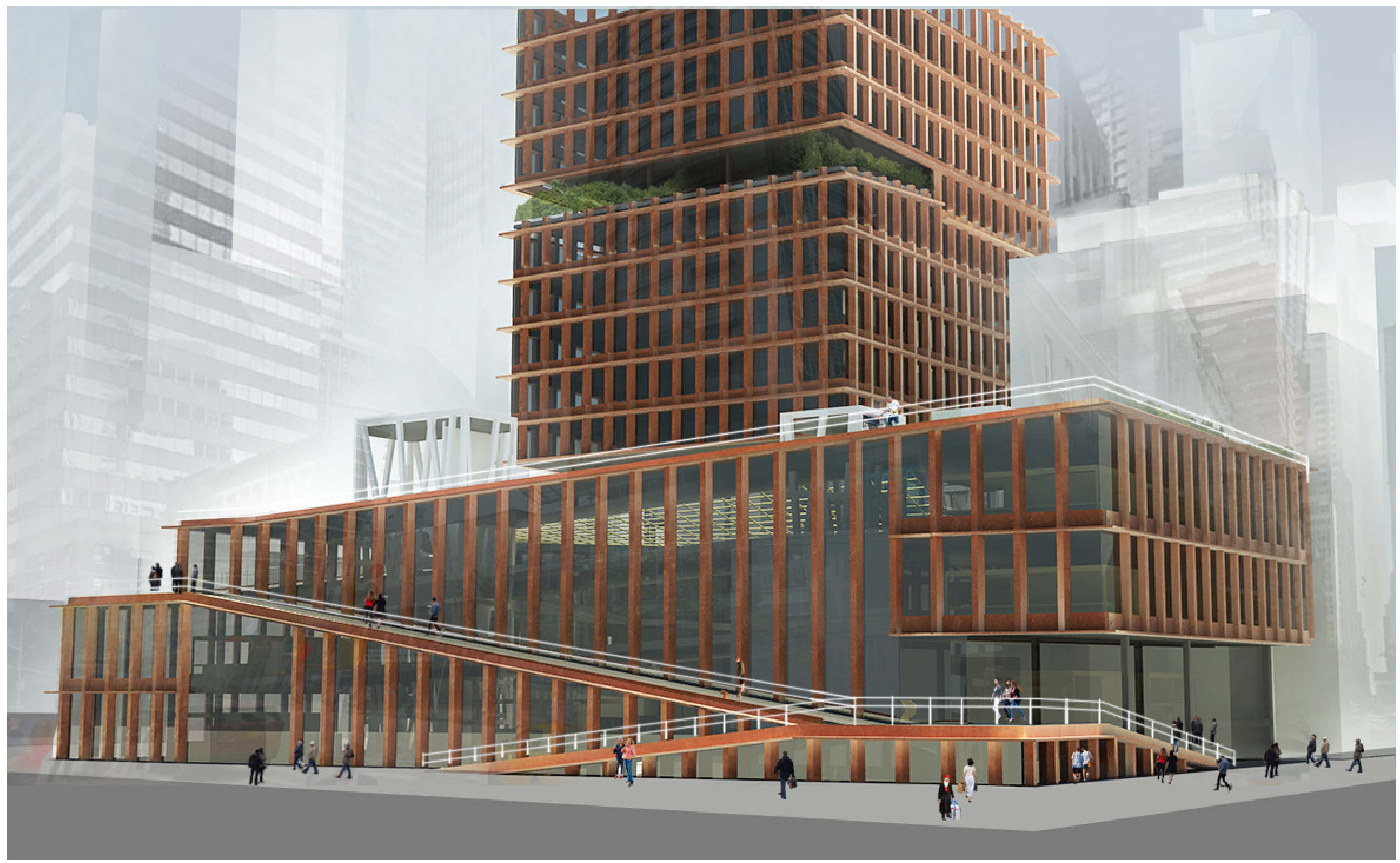




\section{Podium Section}
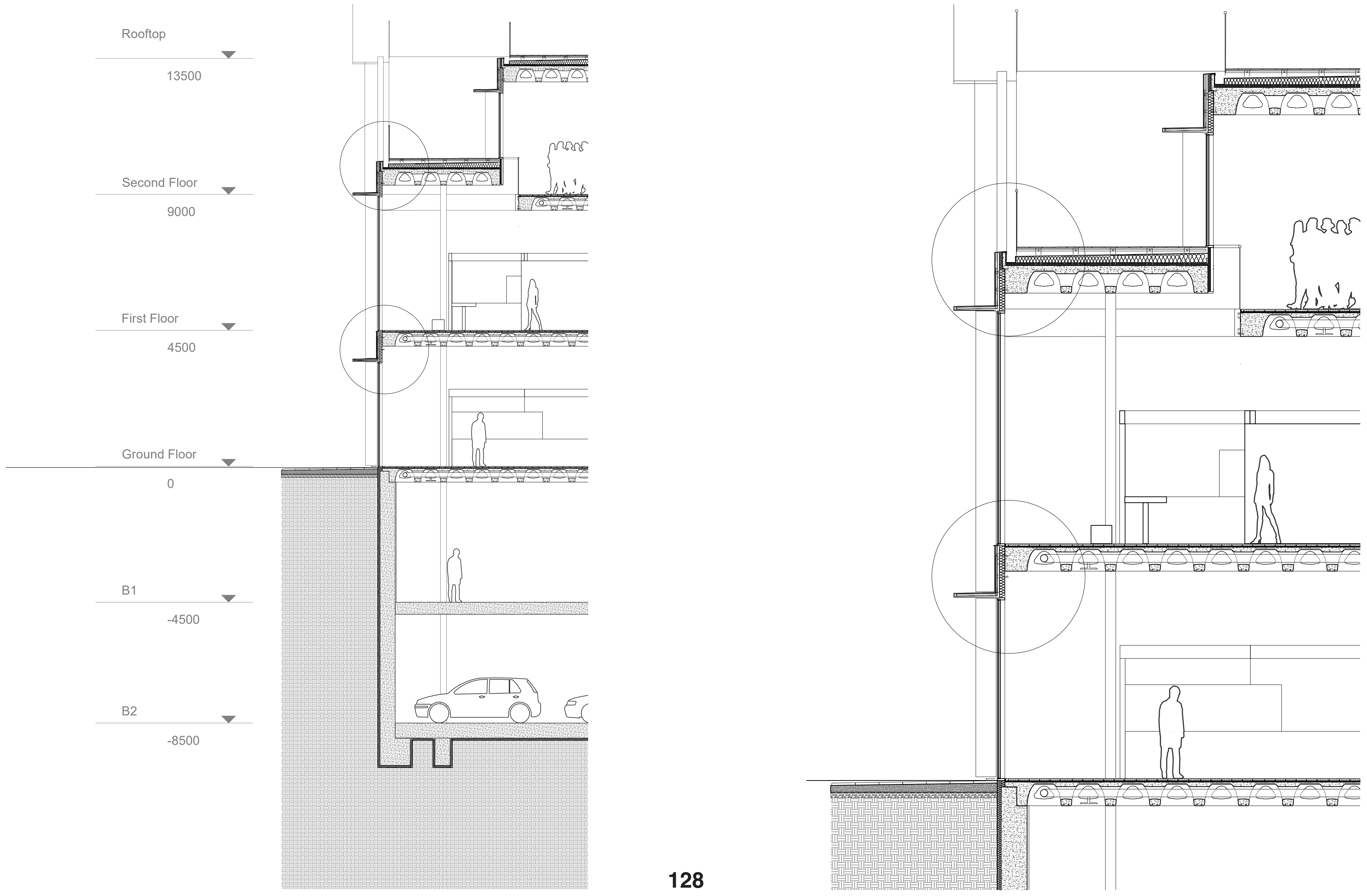


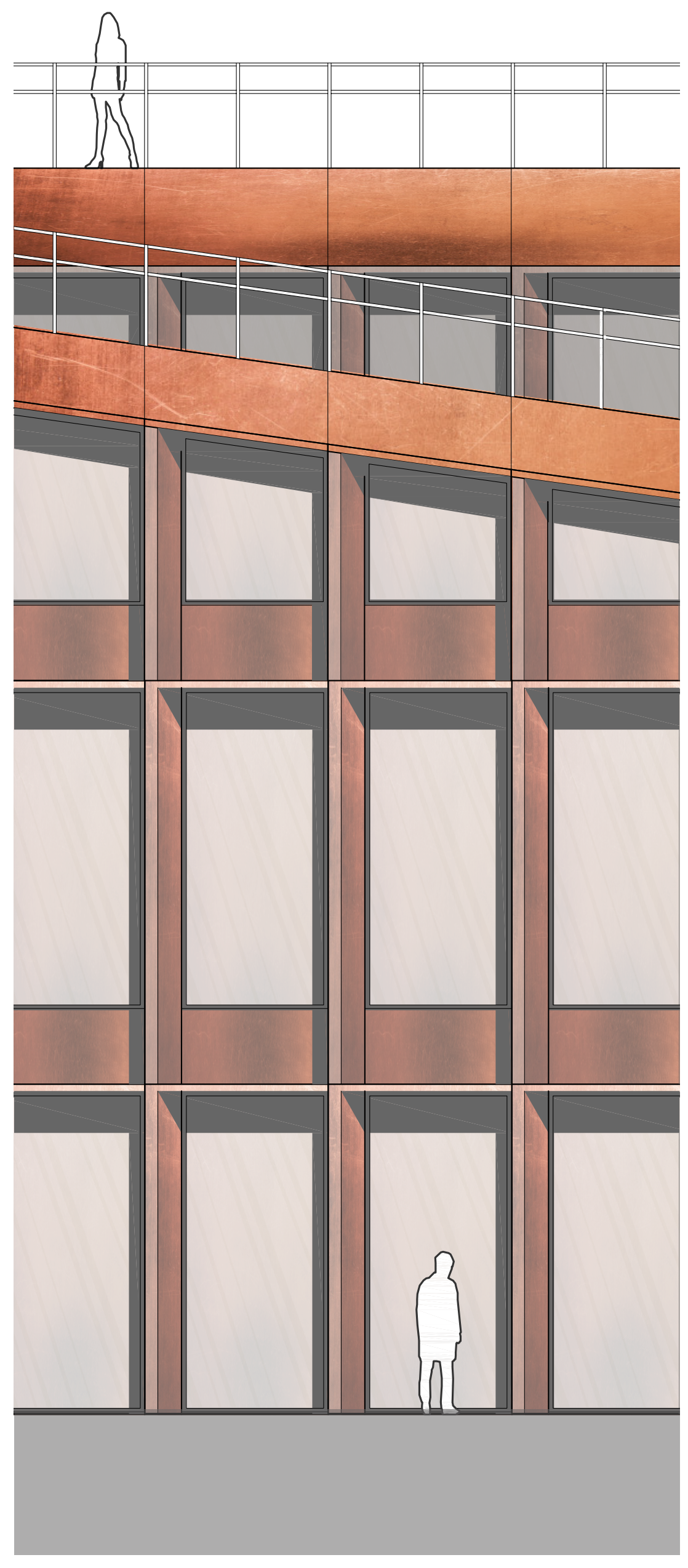

\section{Rooftop}

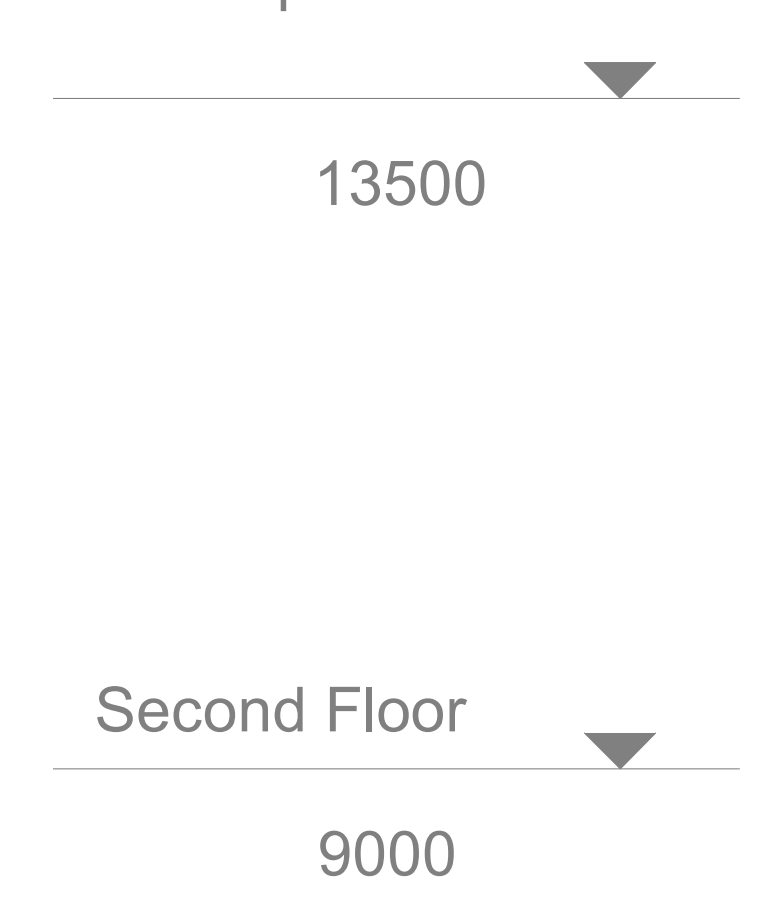

Ground Floor

0
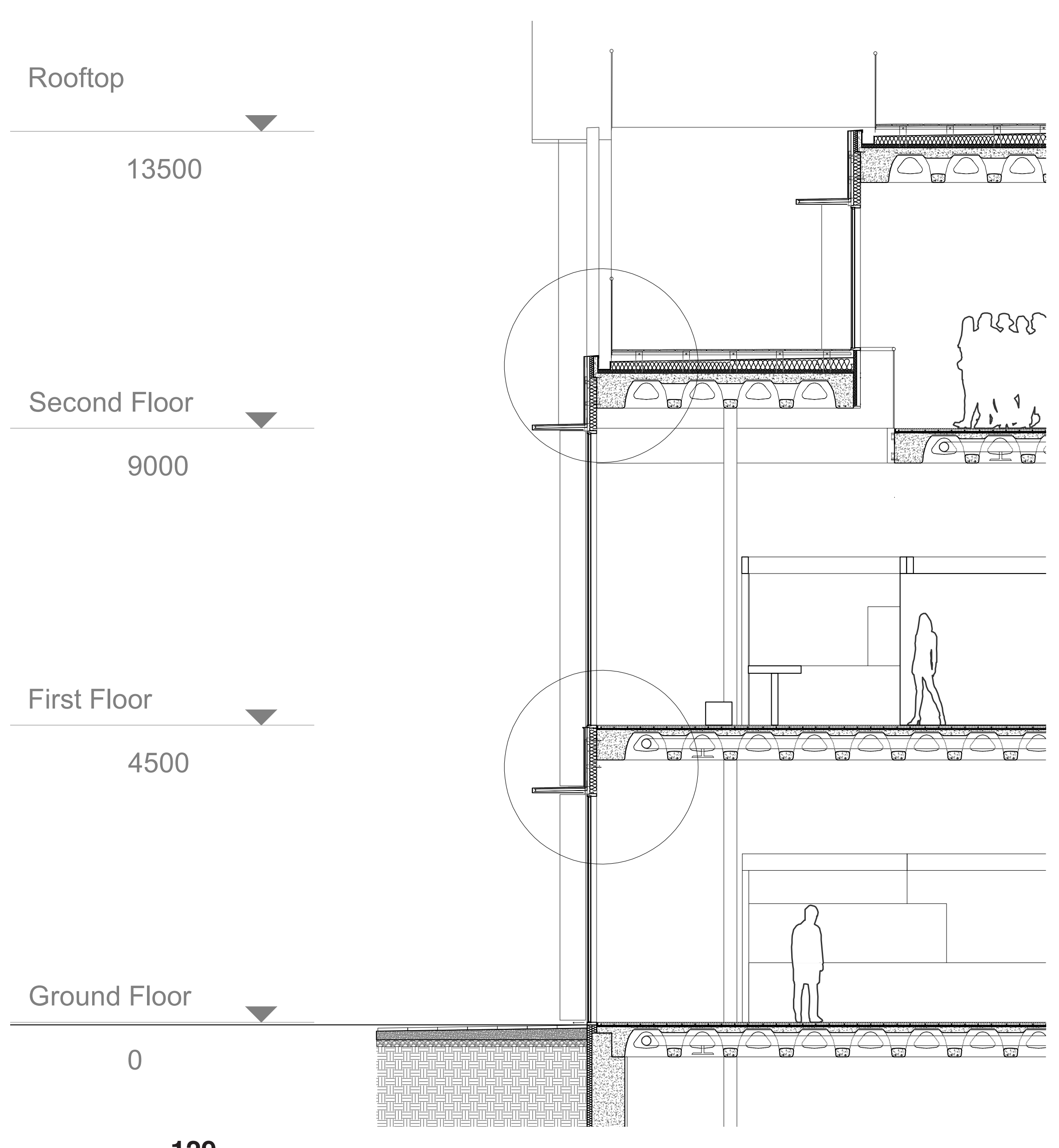


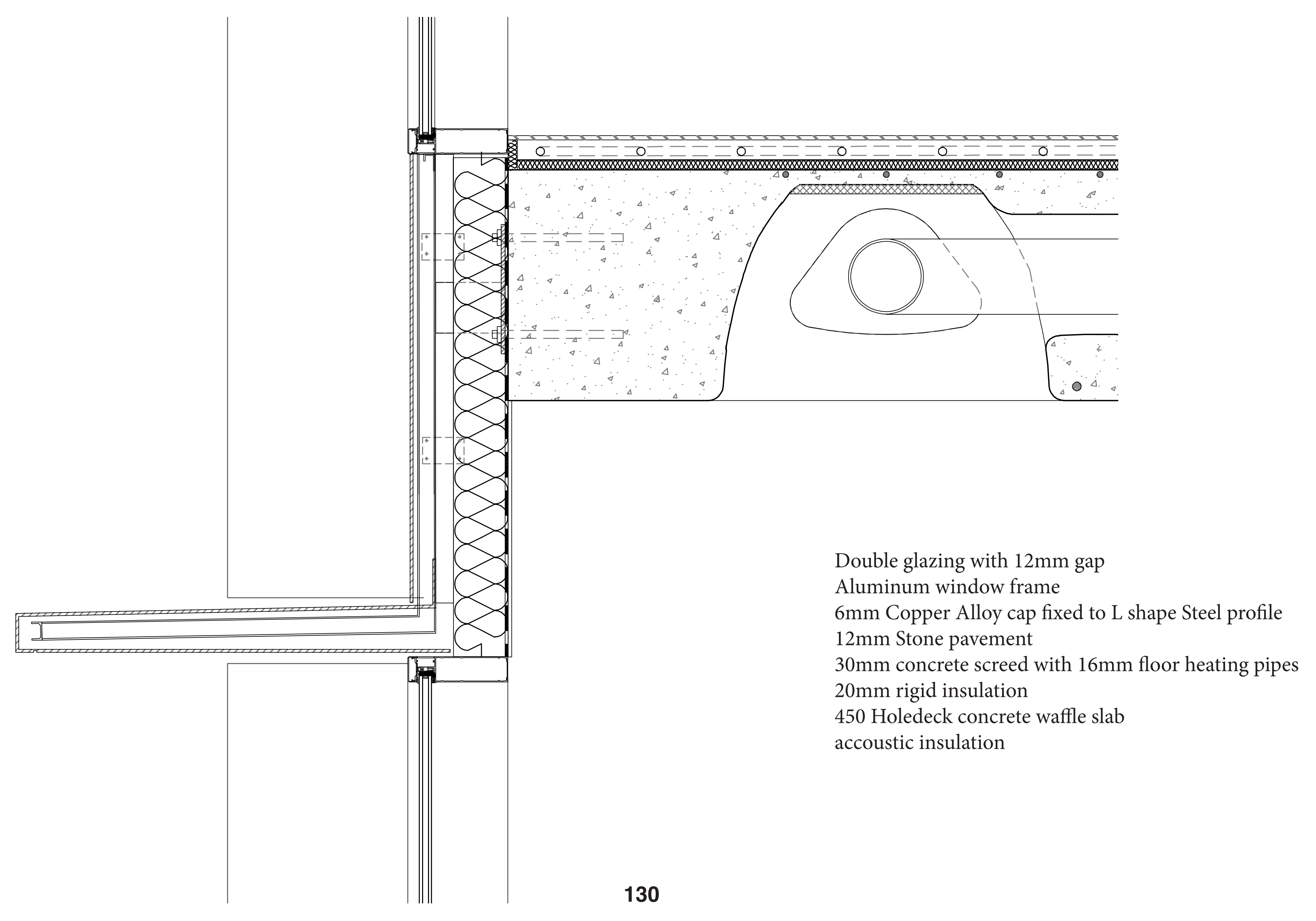




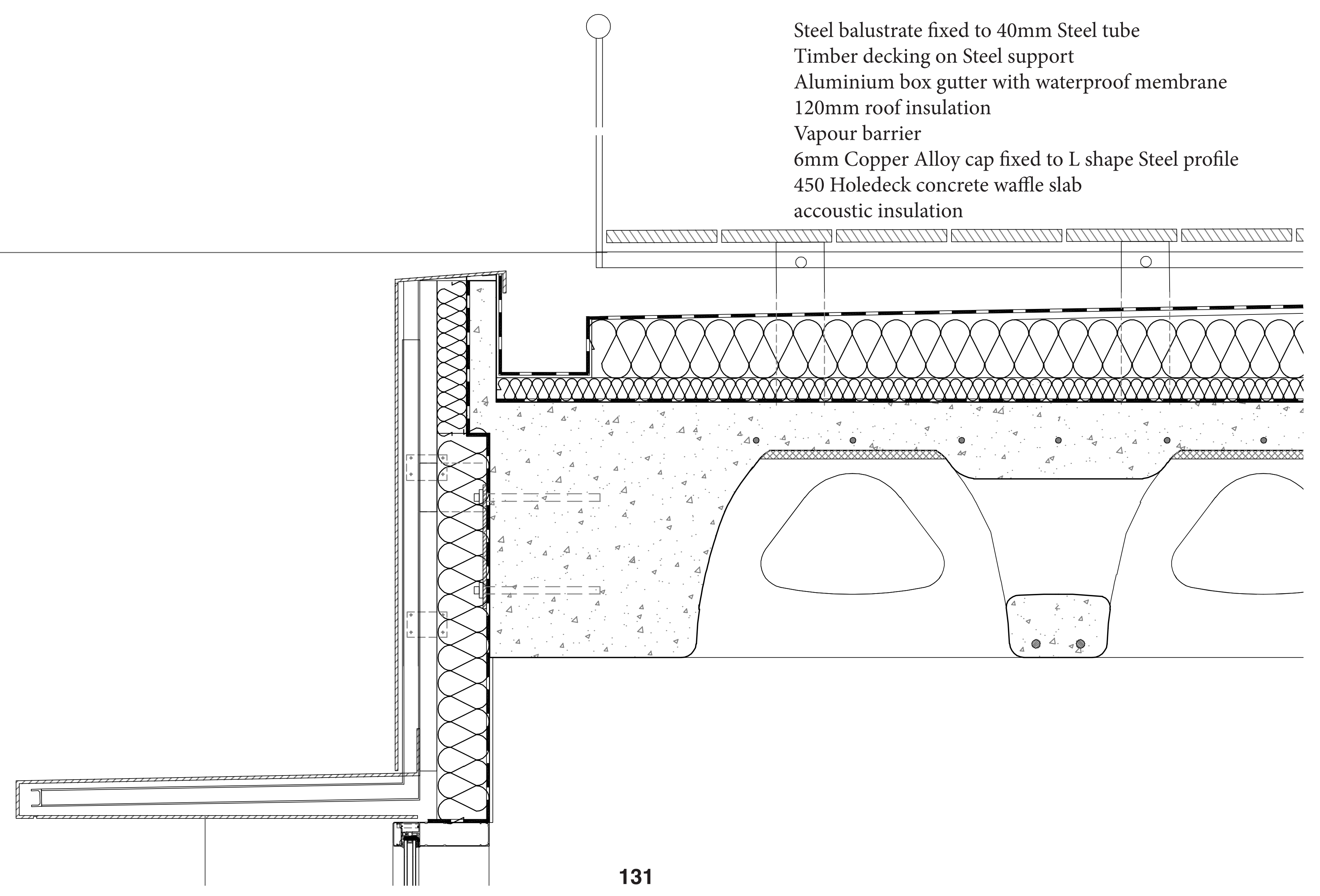


Vegetation

$150 \mathrm{~mm}$ growing medium

Root repelient

Drainage Layer

Waterproof membrane

Insulation Layer

Vapour barrior

$450 \mathrm{~mm}$ concrete waffle slab

accoustic insulation

air ducts

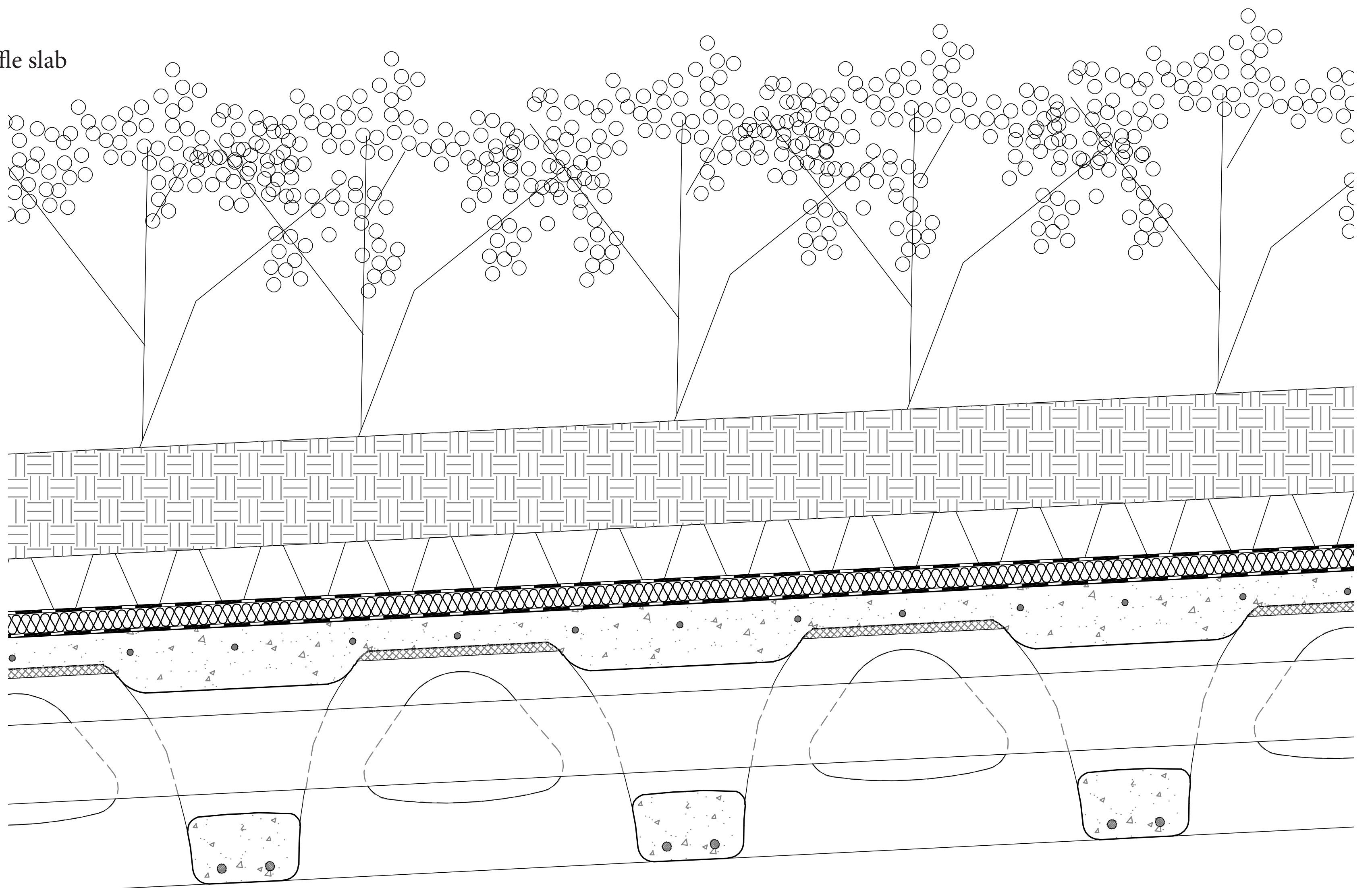




\section{Section drawing}

Residential tower

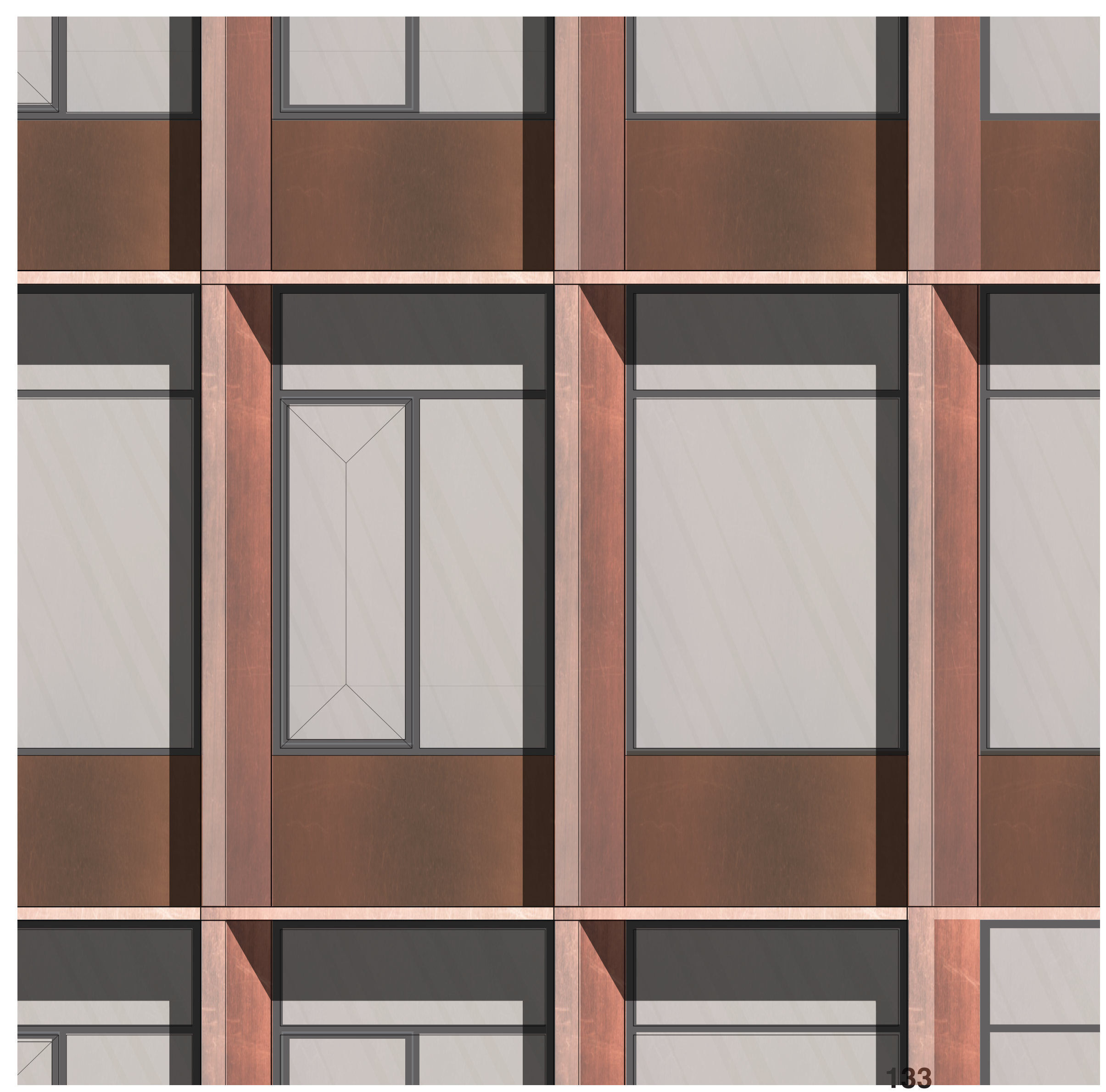

6mm Copper Alloy cap fixed to L shape Steel profile Copper cladding panel

$120 \mathrm{~mm}$ wall insulation

$12 \mathrm{~mm}$ phase changing gypsum board

interior carpet

$30 \mathrm{~mm}$ concrete screed with floor heating pipes

$20 \mathrm{~mm}$ rigid insulation

$30 \mathrm{~mm}$ in situ concrete screed

$300 \mathrm{~mm}$ hollowicore concrete slabs fixed to $300 \mathrm{~mm}$ steel PFC beam Suspended ceilling
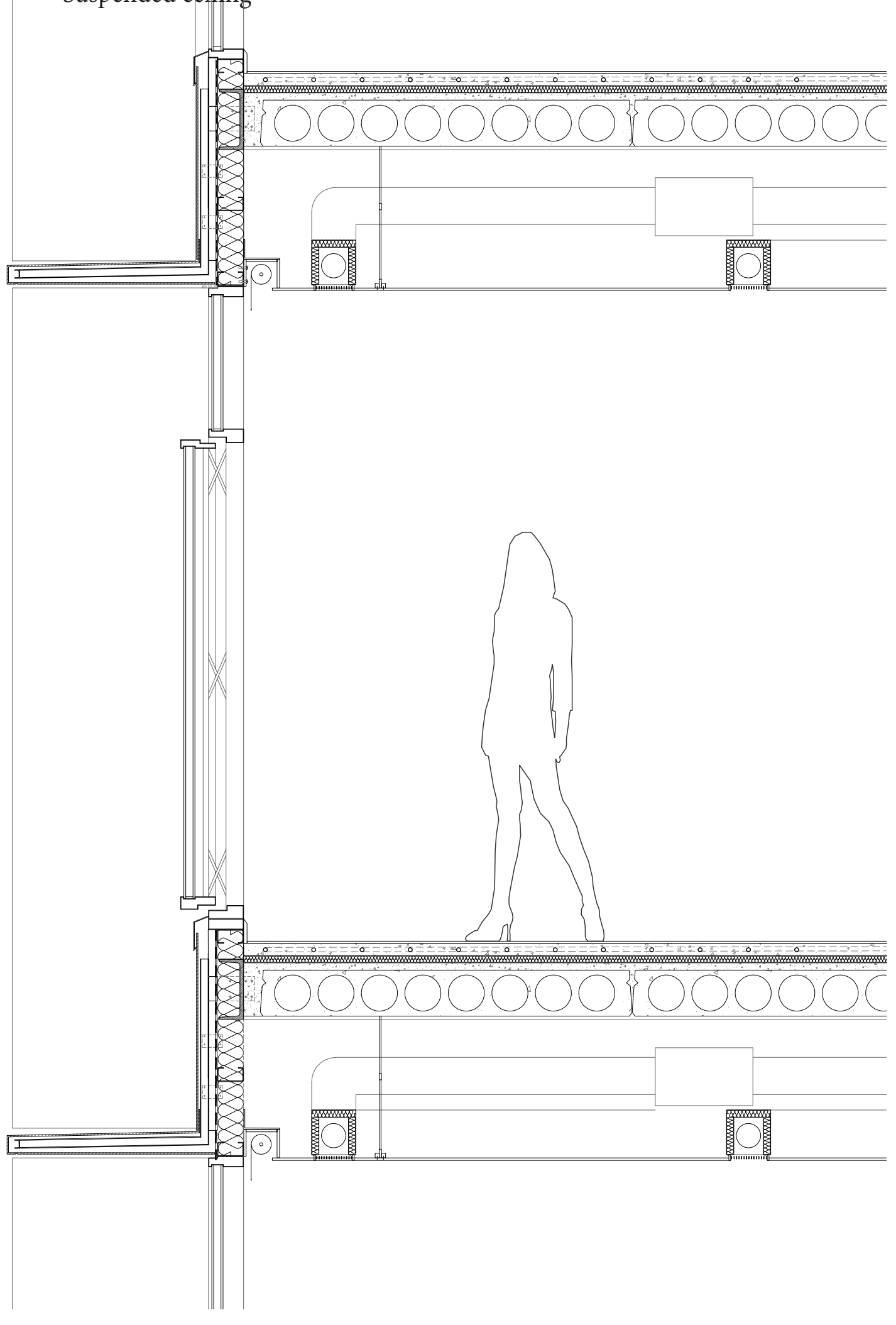


\section{Section drawing}

Residential tower

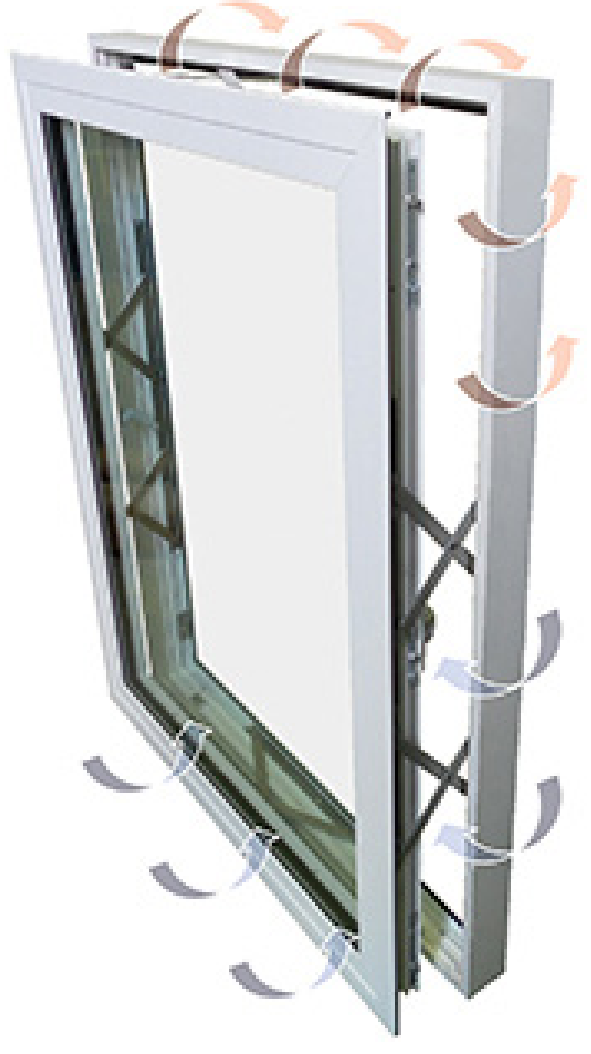

Parallel opening window

-small opening movement with better air circulation -good accoustic performance -reducing draughts
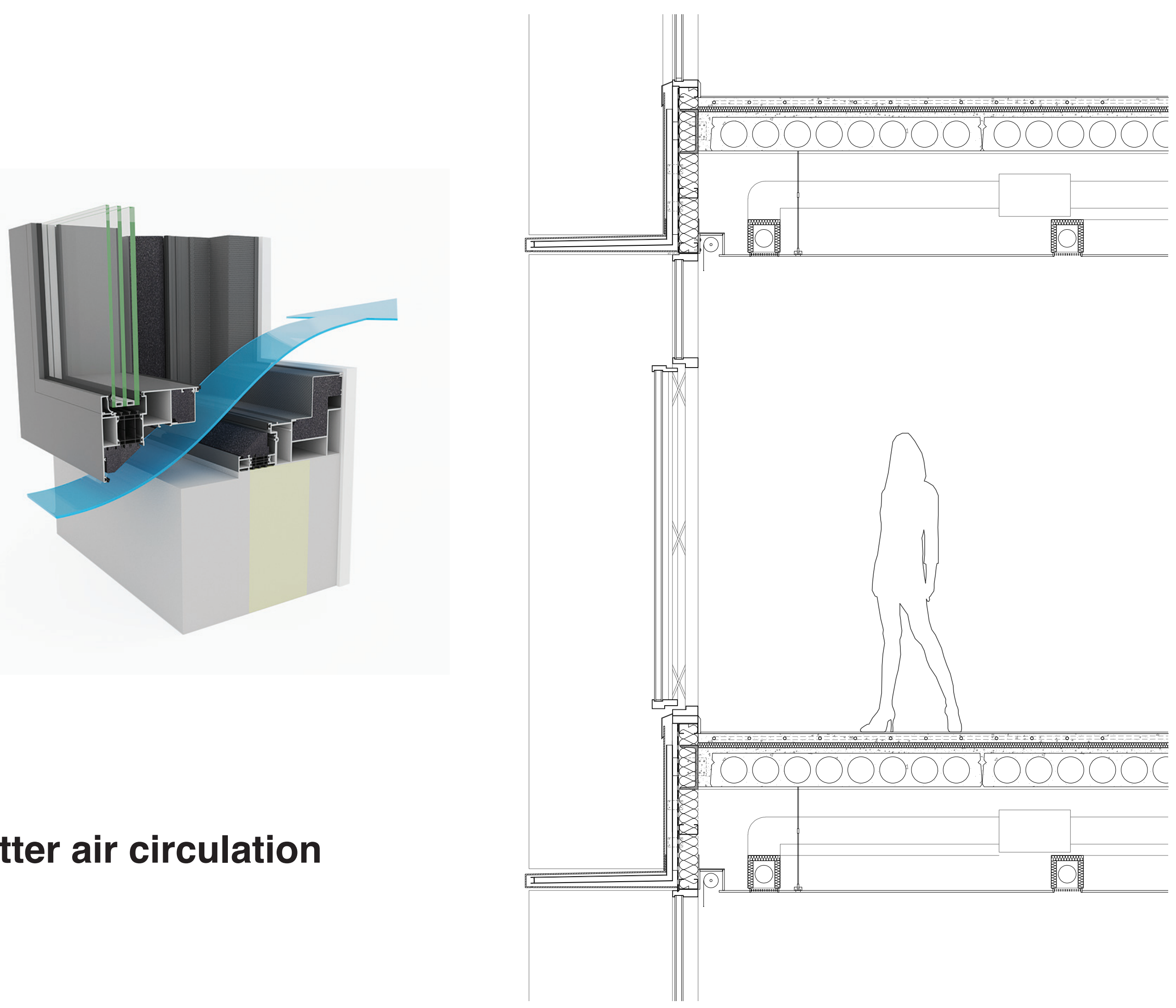
$120 \mathrm{~mm}$ wall

$12 \mathrm{~mm}$ phase changing gypsum board

interior carpet

$30 \mathrm{~mm}$ concrete screed with floor heating pipes

$20 \mathrm{~mm}$ rigid insulation

$30 \mathrm{~mm}$ in situ concrete screed

$300 \mathrm{~mm}$ hollowcore concrete slabs fixed to $300 \mathrm{~mm}$ steel PFC beam

Suspended ceiling

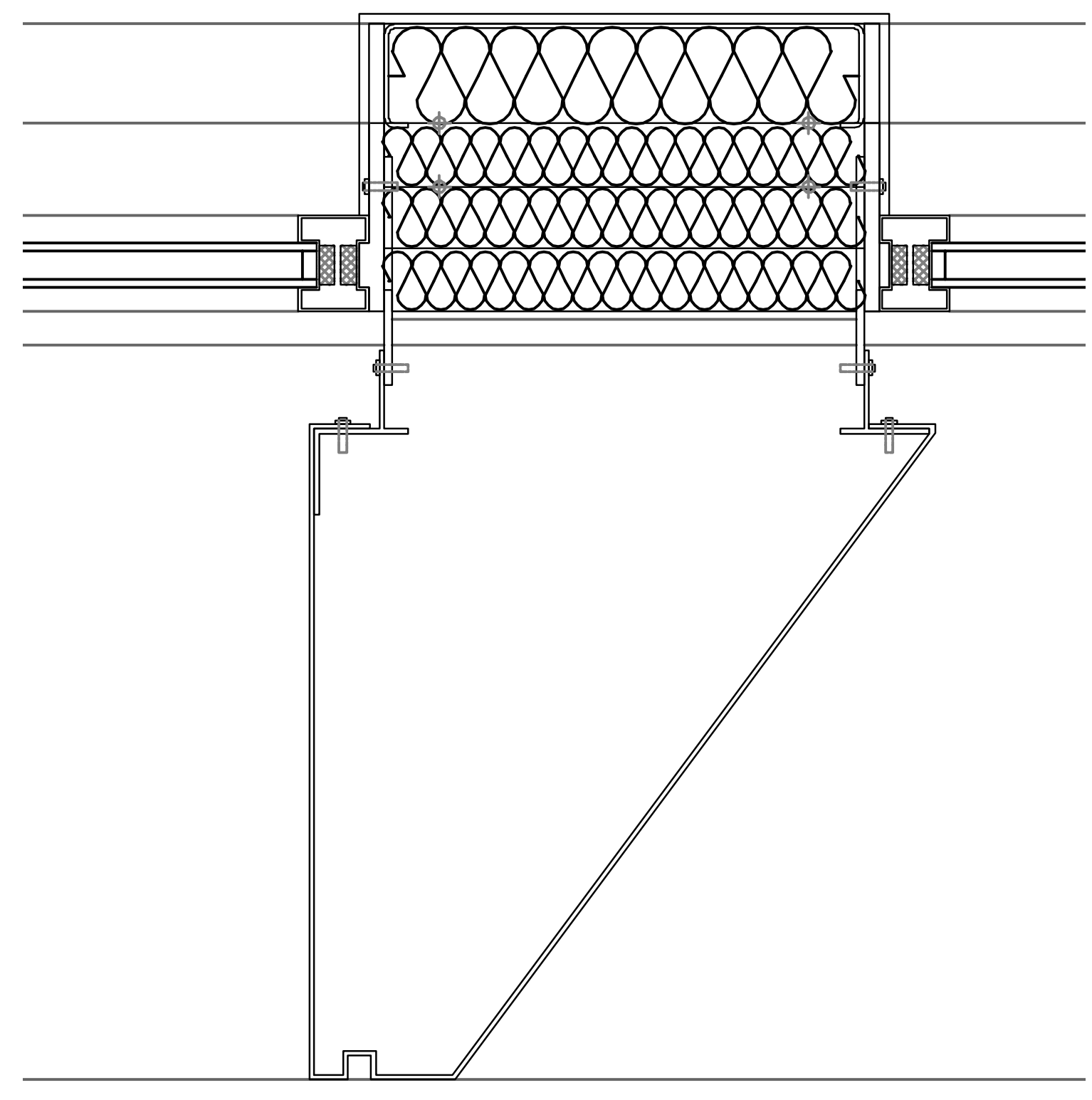




\section{Structural Concept}


Structural Scheme

Podium

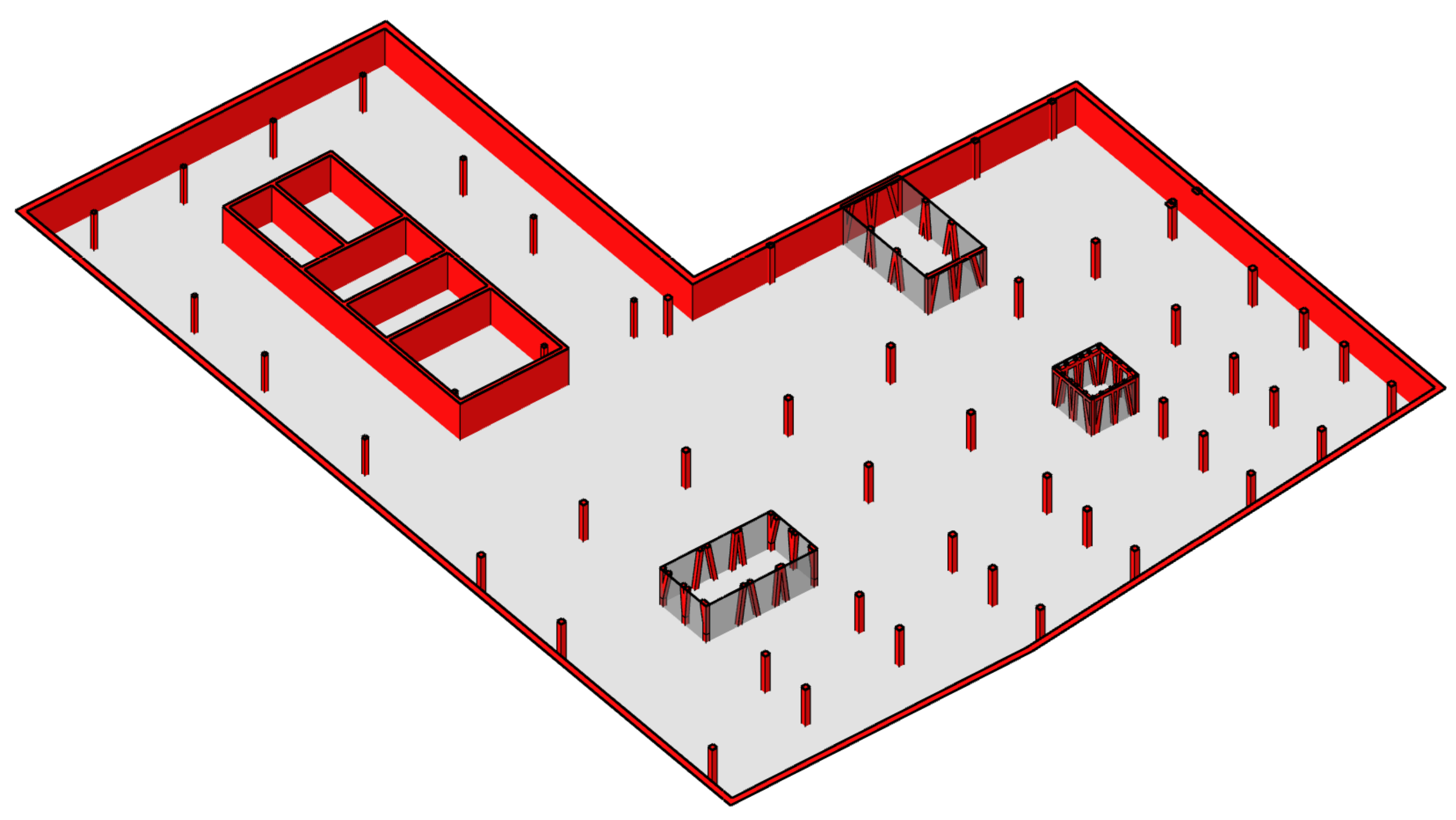




\section{Structural Scheme}

Podium

steel vertical cores supporting the interior slabs and ramps and provide stability

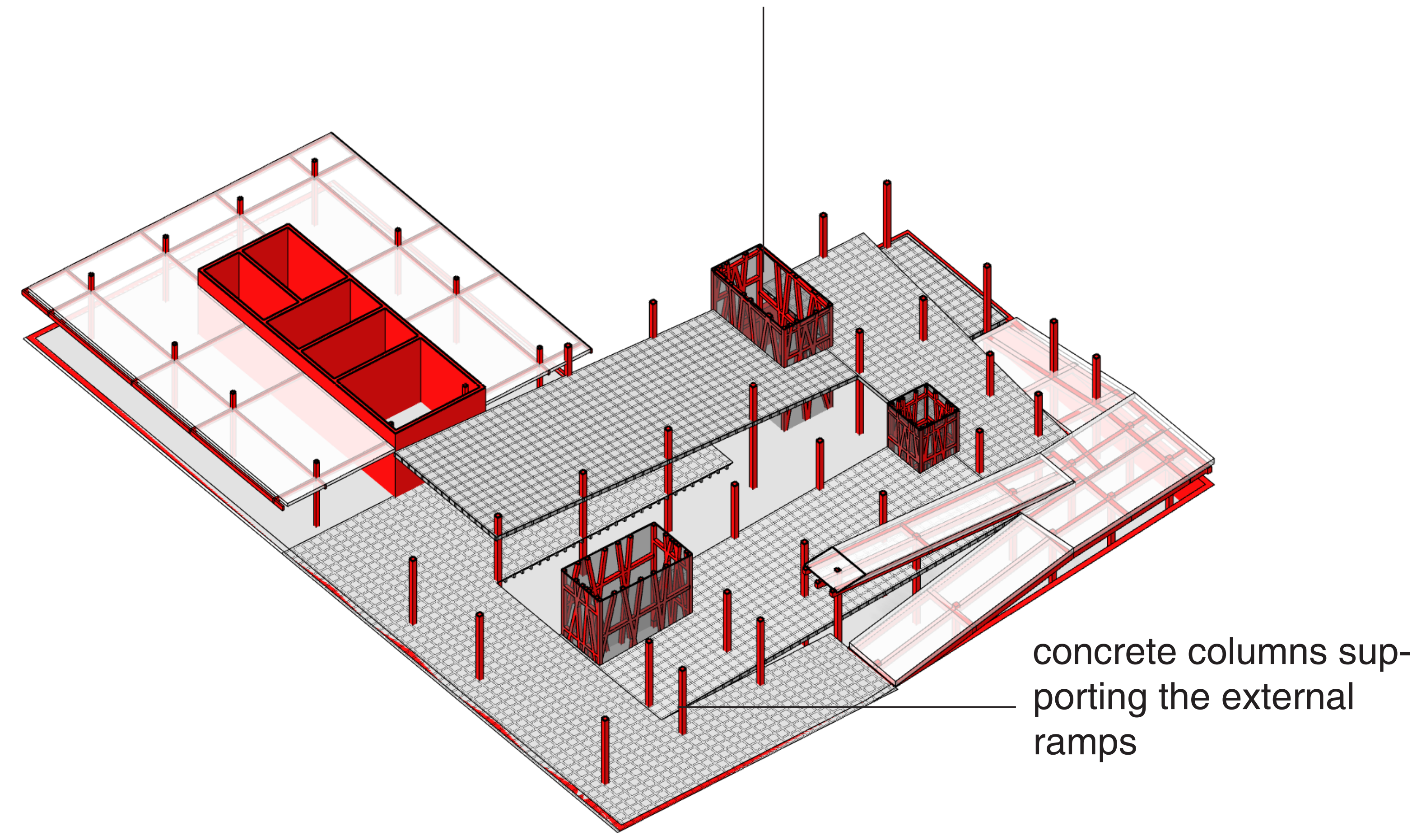


Structural Scheme

Podium

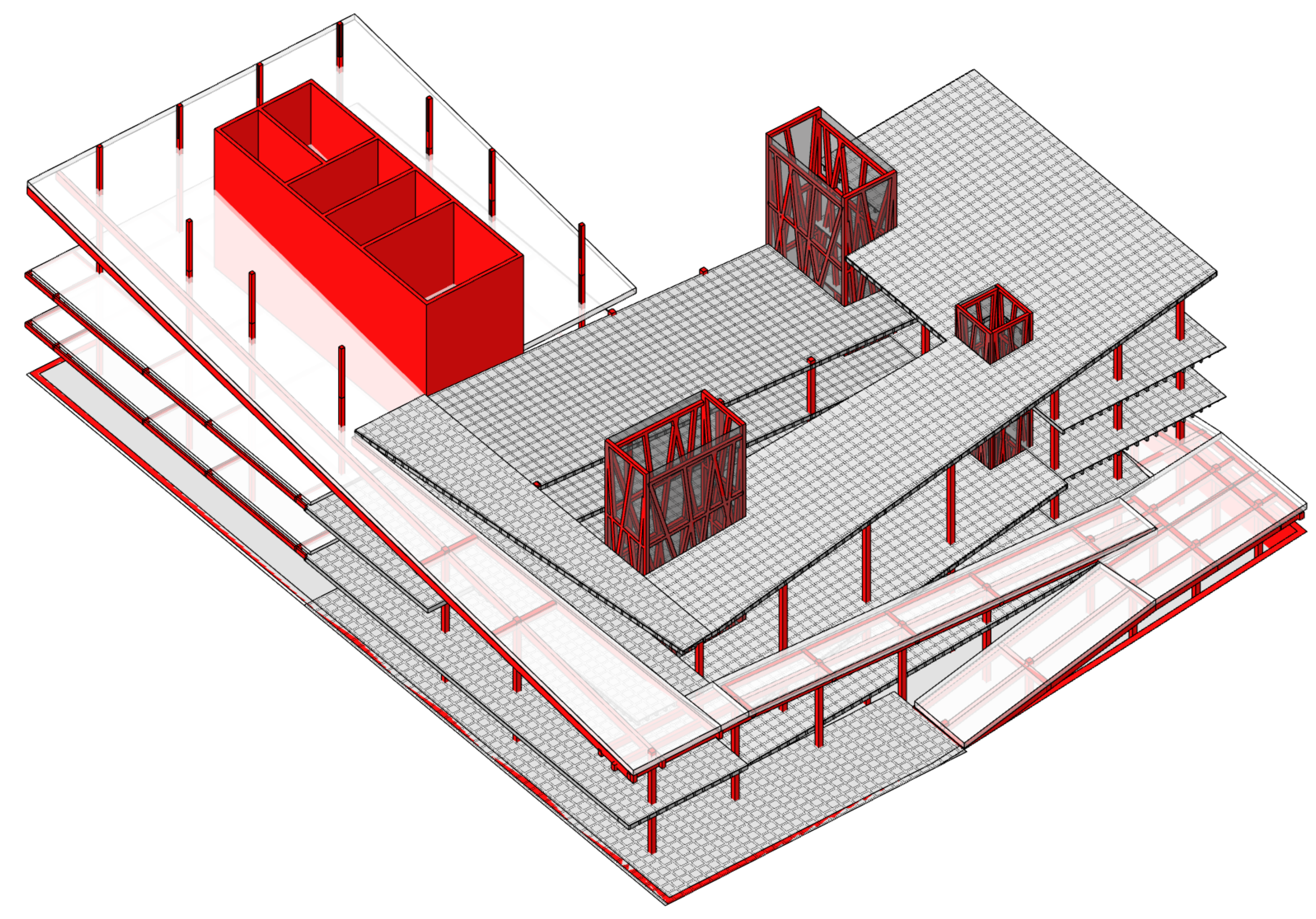




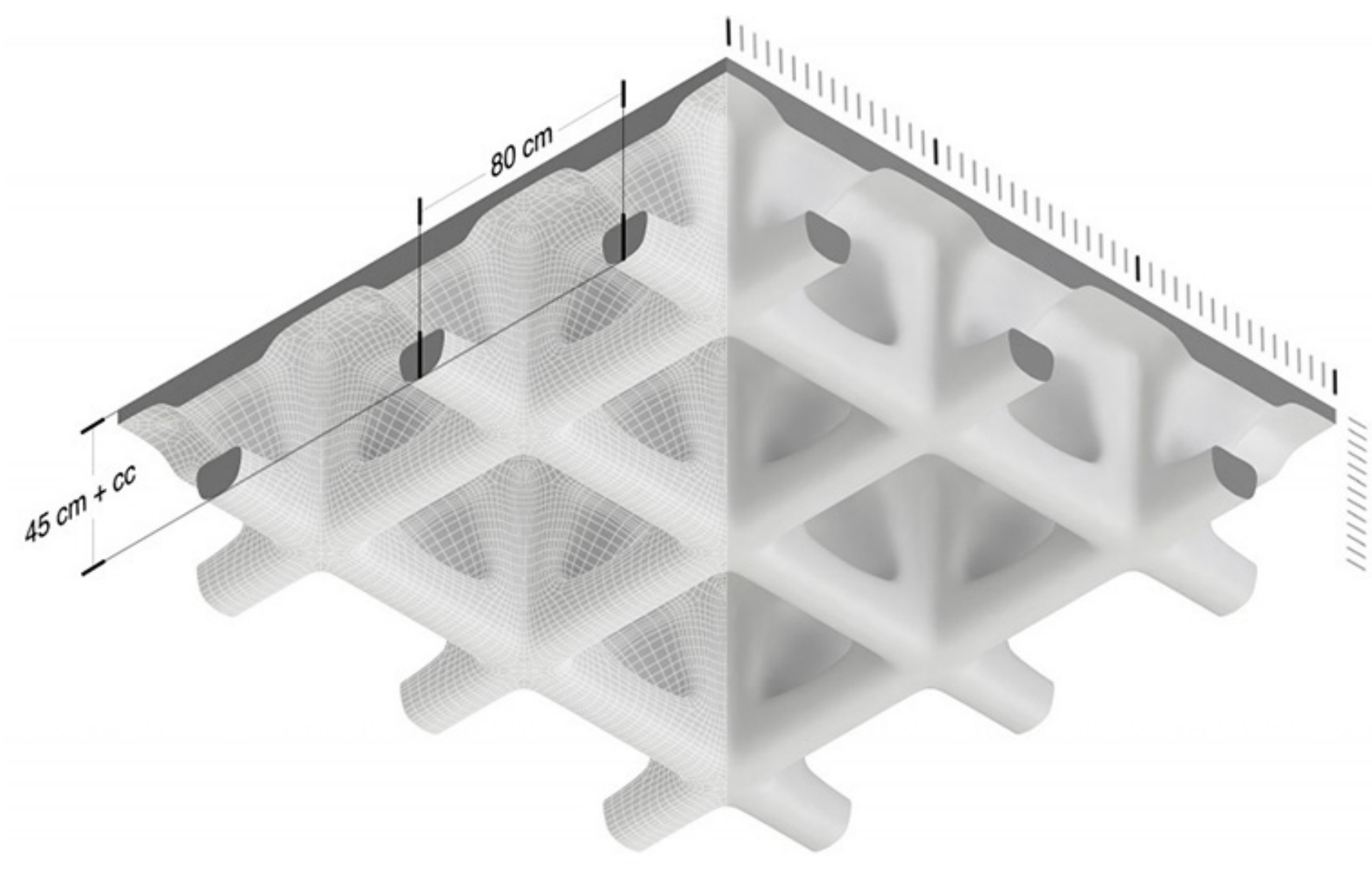

\section{Lightweight}

Span between $8 \mathrm{~m}$ to $13 \mathrm{~m}$

Spatial Efficiency

Easy maintainence

Low embodied energy 


\section{Structural Scheme}

\section{Podium}

Steel section to floor detail

$8 \mathrm{~mm}$ fireproofing laminated Wglass

stone pavement

$30 \mathrm{~mm}$ concrete screed with $16 \mathrm{~mm}$ heating/cooling pipes

$20 \mathrm{~mm}$ rigid insulation

$300 \mathrm{~mm}$ PFC beam fixed to $450 \mathrm{~mm}$ steel waffle slab

$20 \mathrm{~mm}$ fire protection board
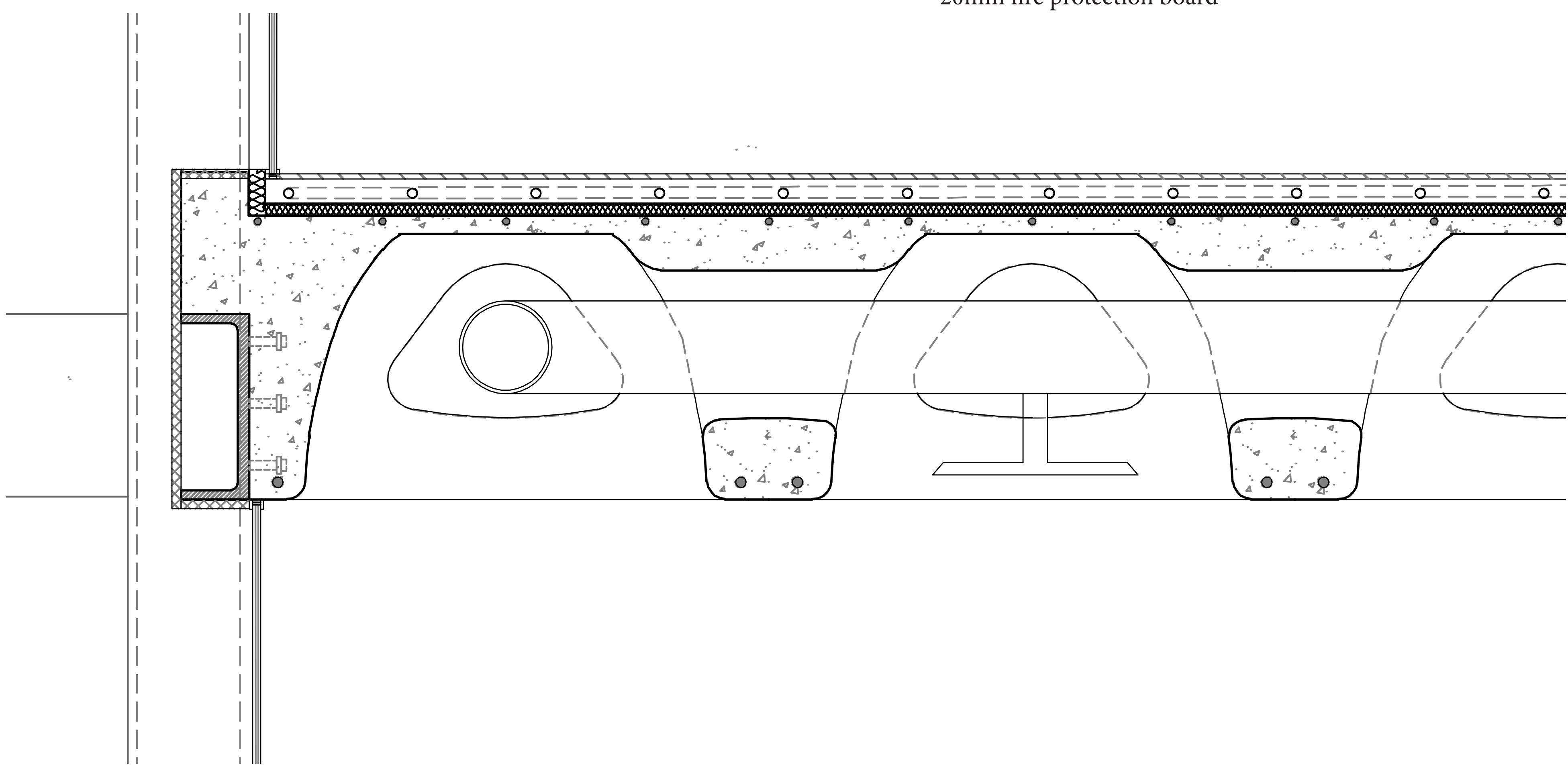


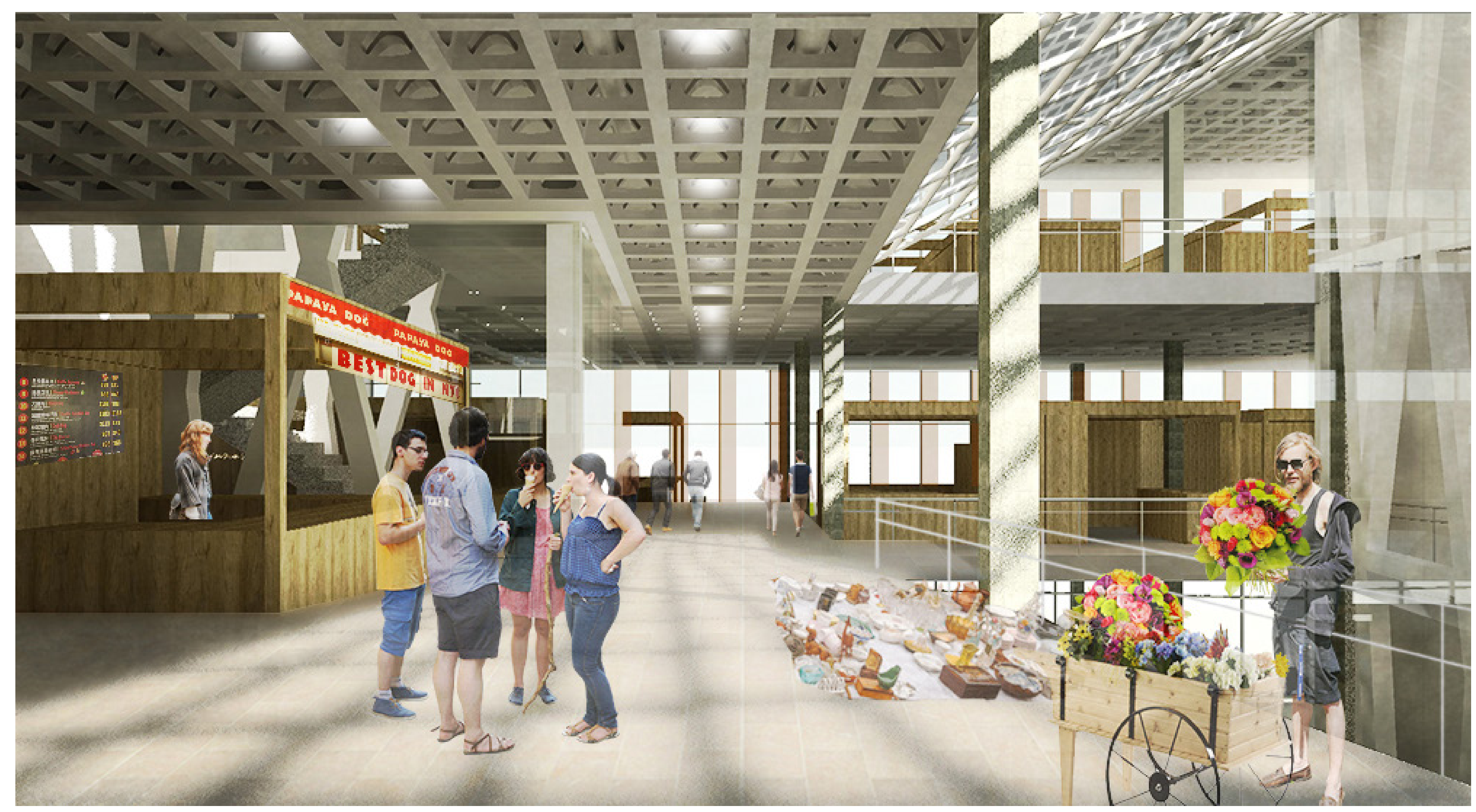


Structural Scheme

Tower
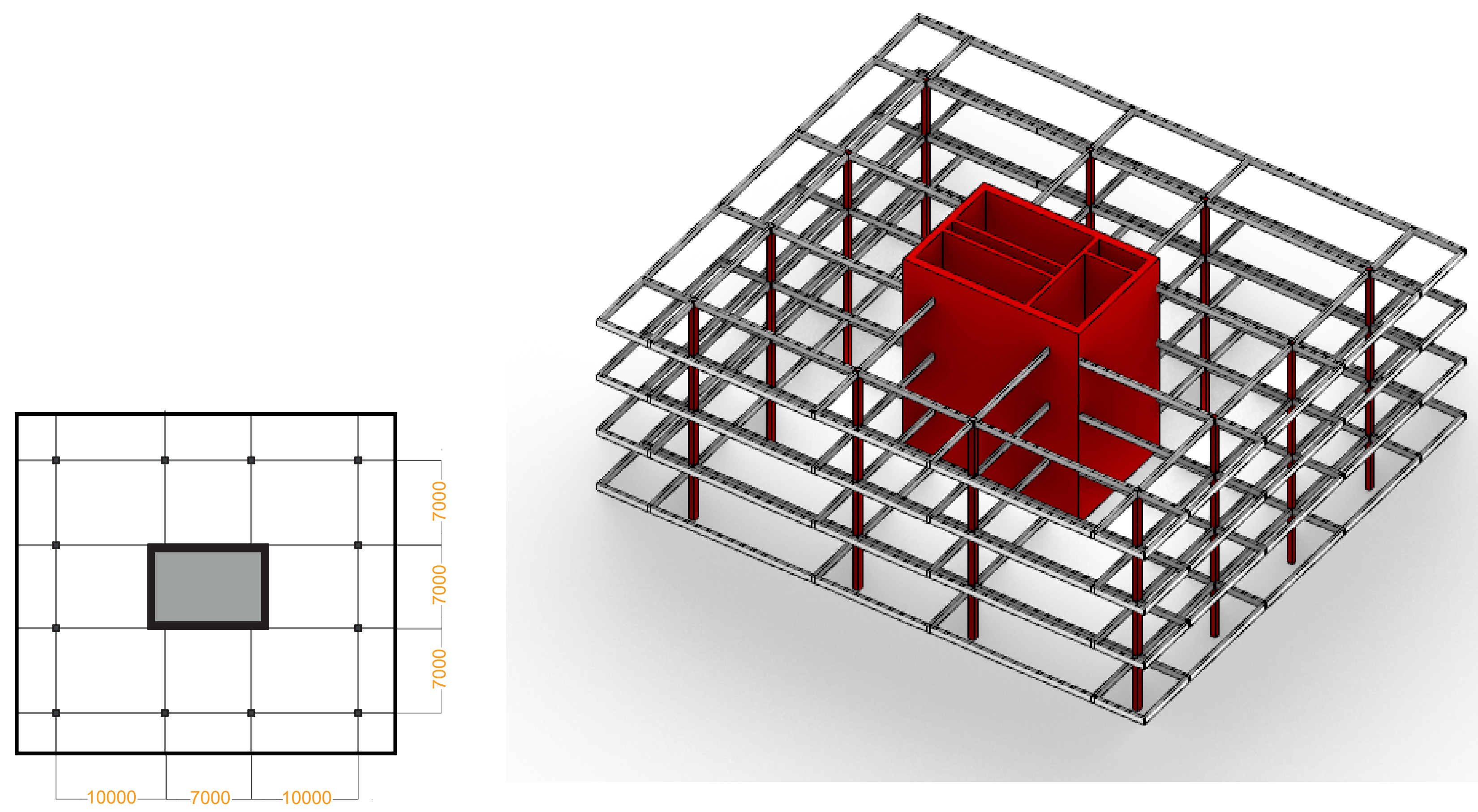


\section{Climatization Concept}




\section{Climate Scheme}

Podium
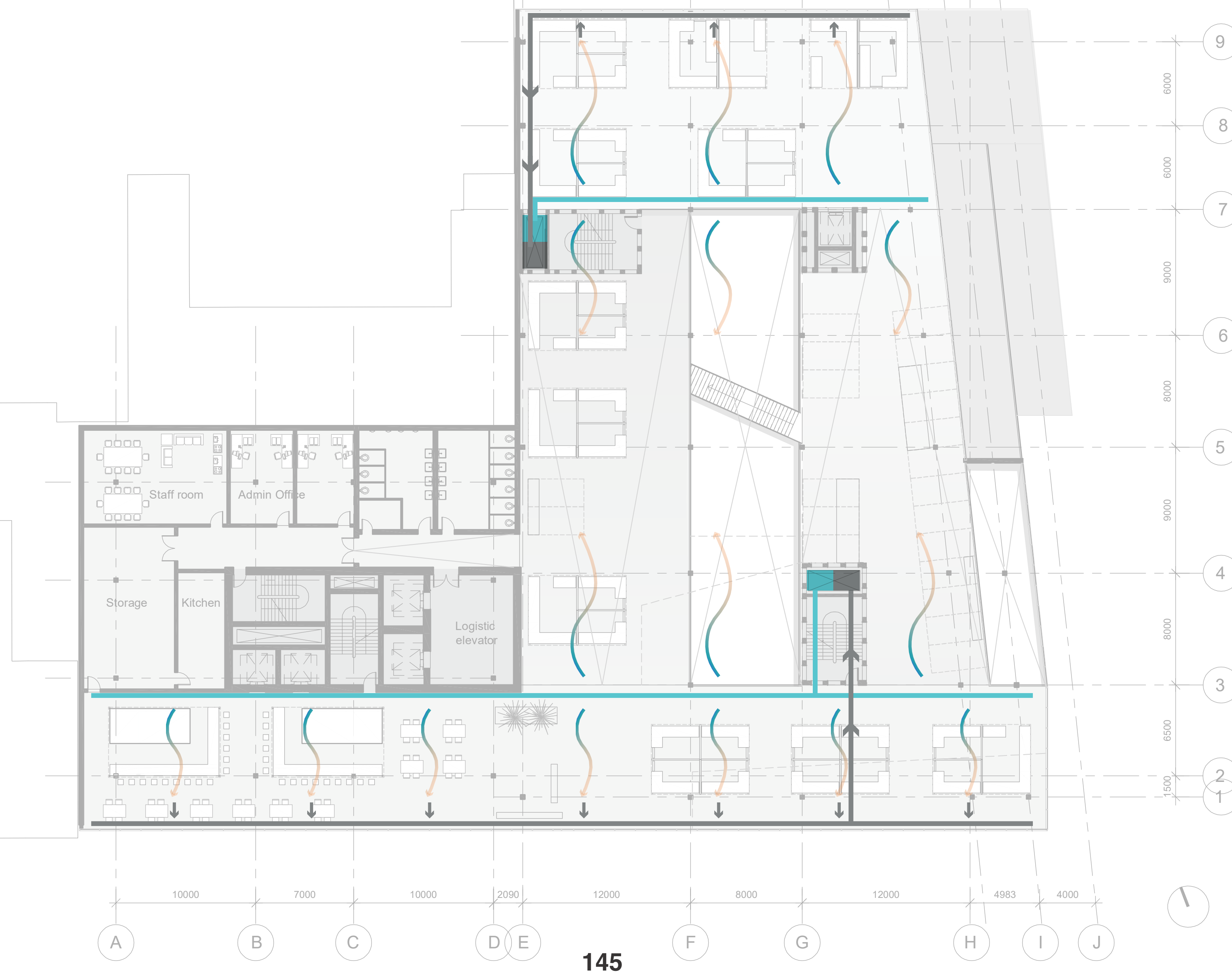


\section{Climate Scheme}

Podium

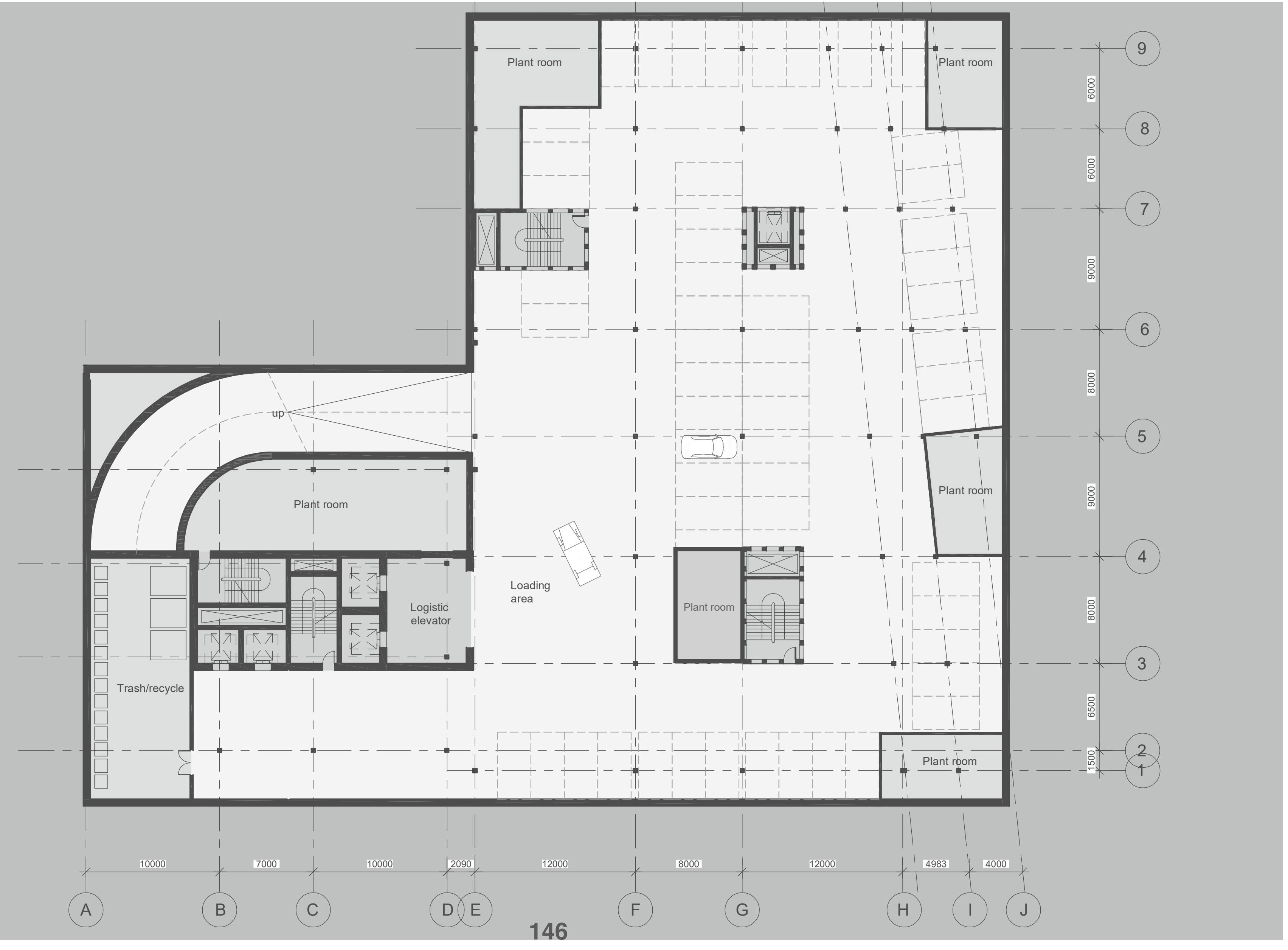




\section{Climate Scheme \\ Podium}

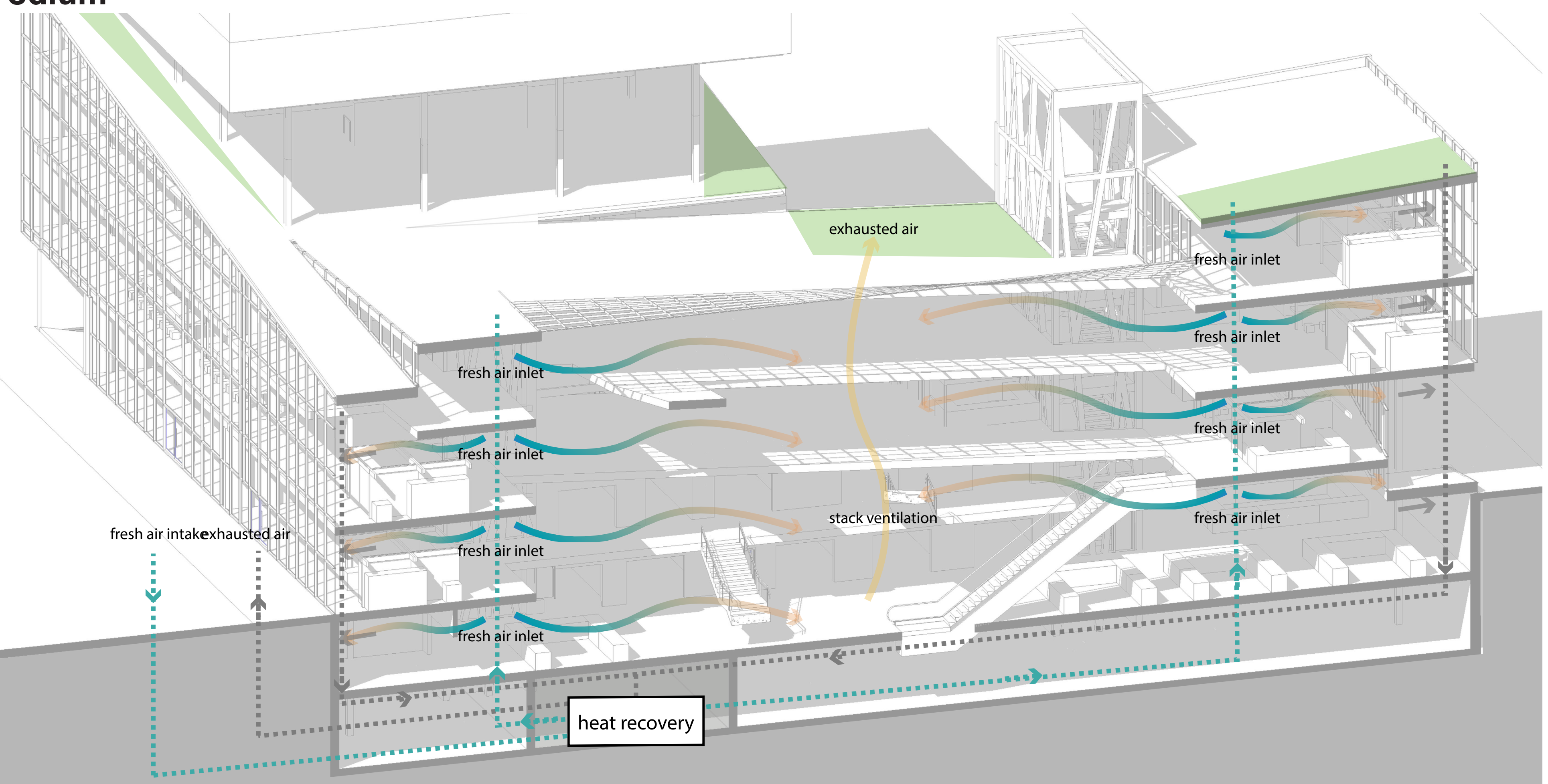




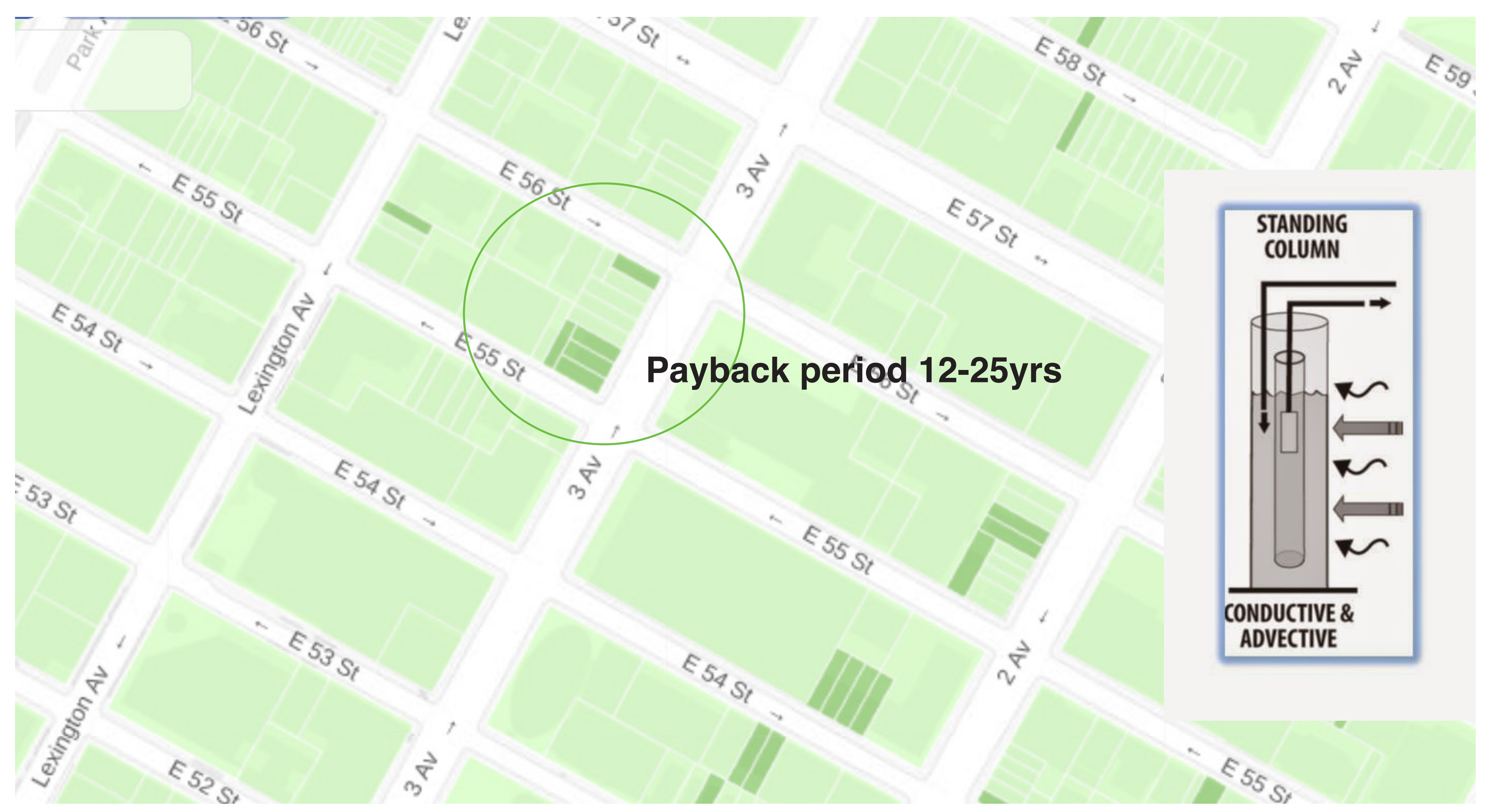

Standing column well system Type of geothermal heat pump system that also uses ground water, but relies on smaller amounts inside a very deep well to exchange heat with the surrounding bedrock. 


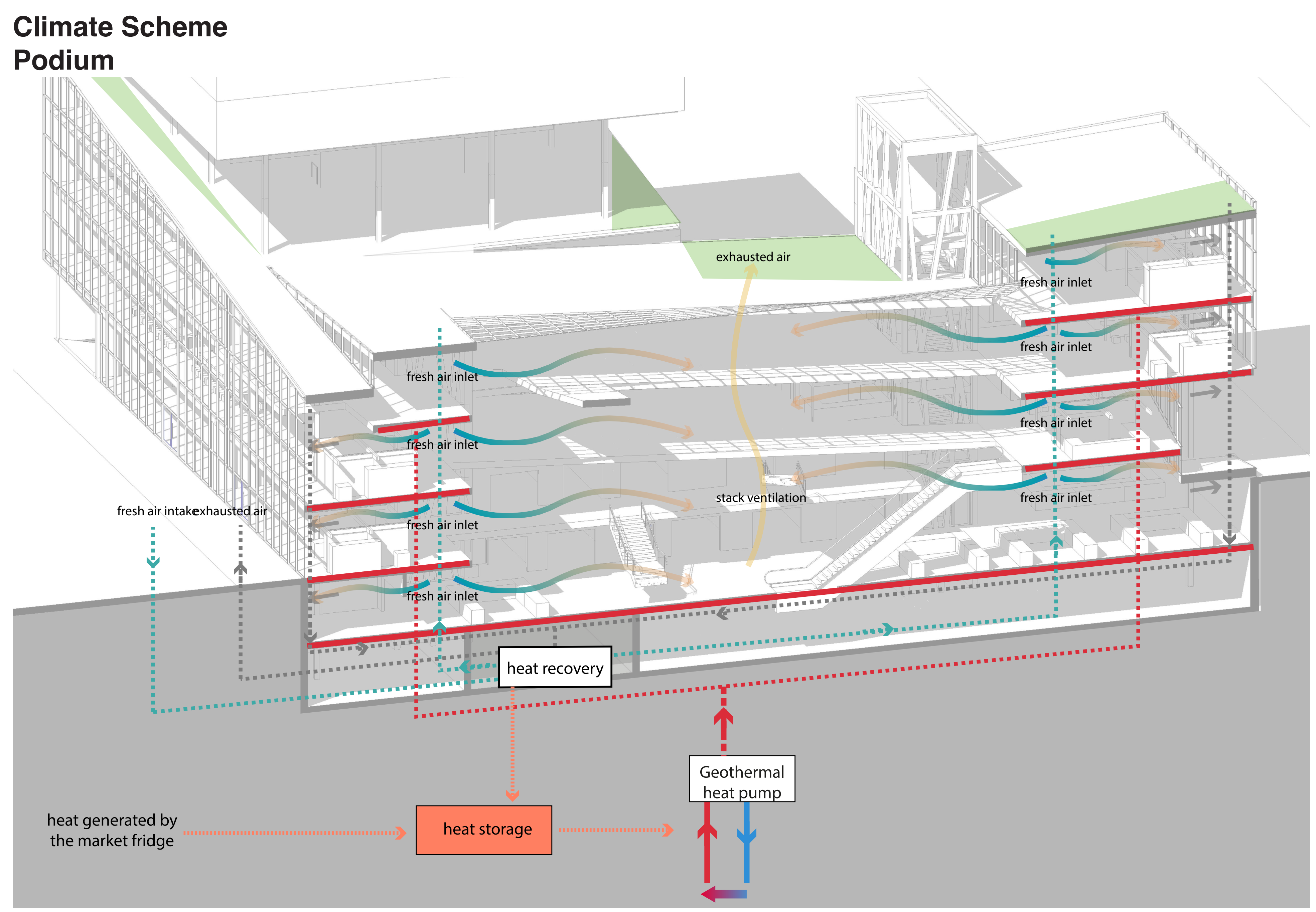




\section{Climate Scheme}

Podium

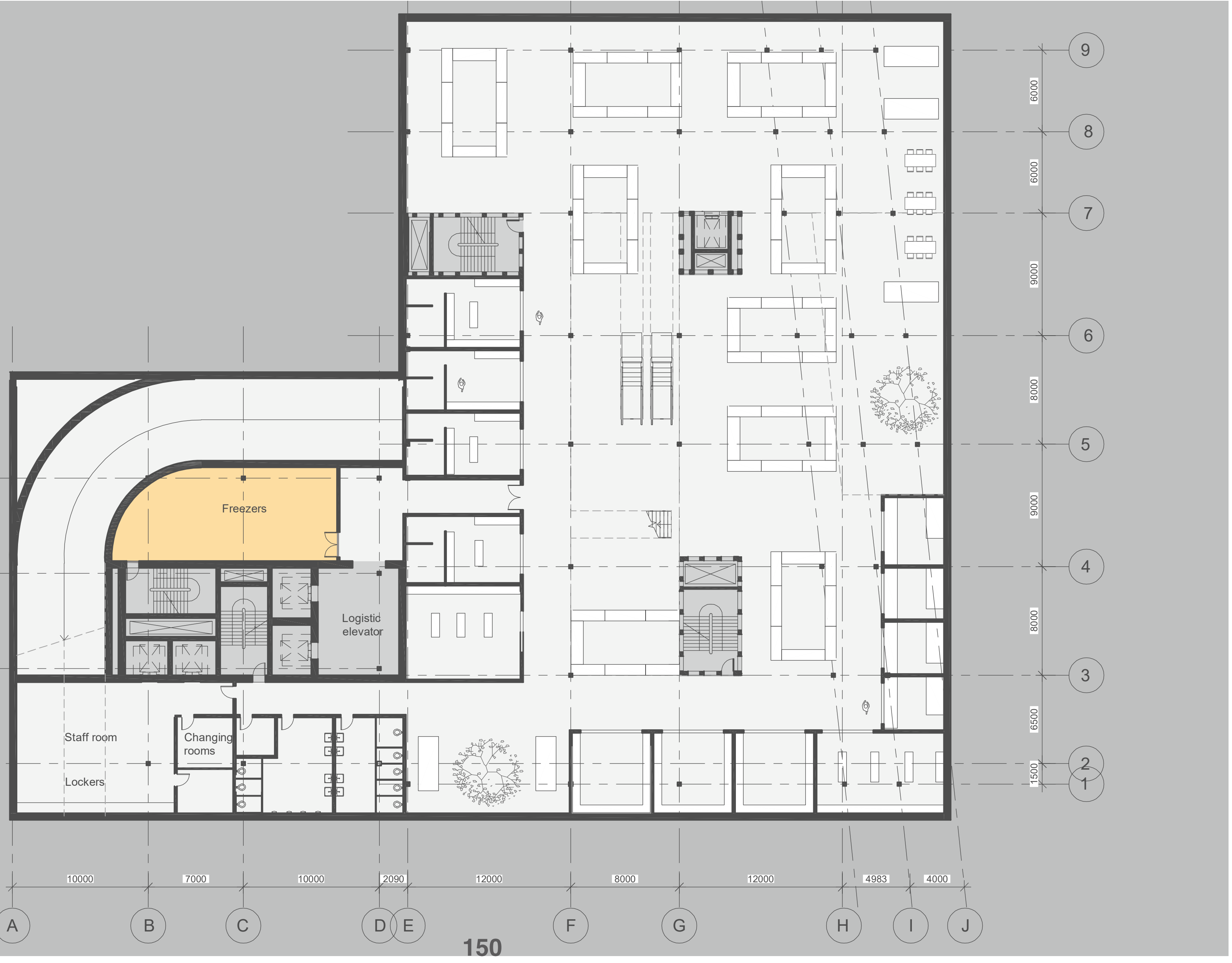




\section{Climate Scheme}

Podium

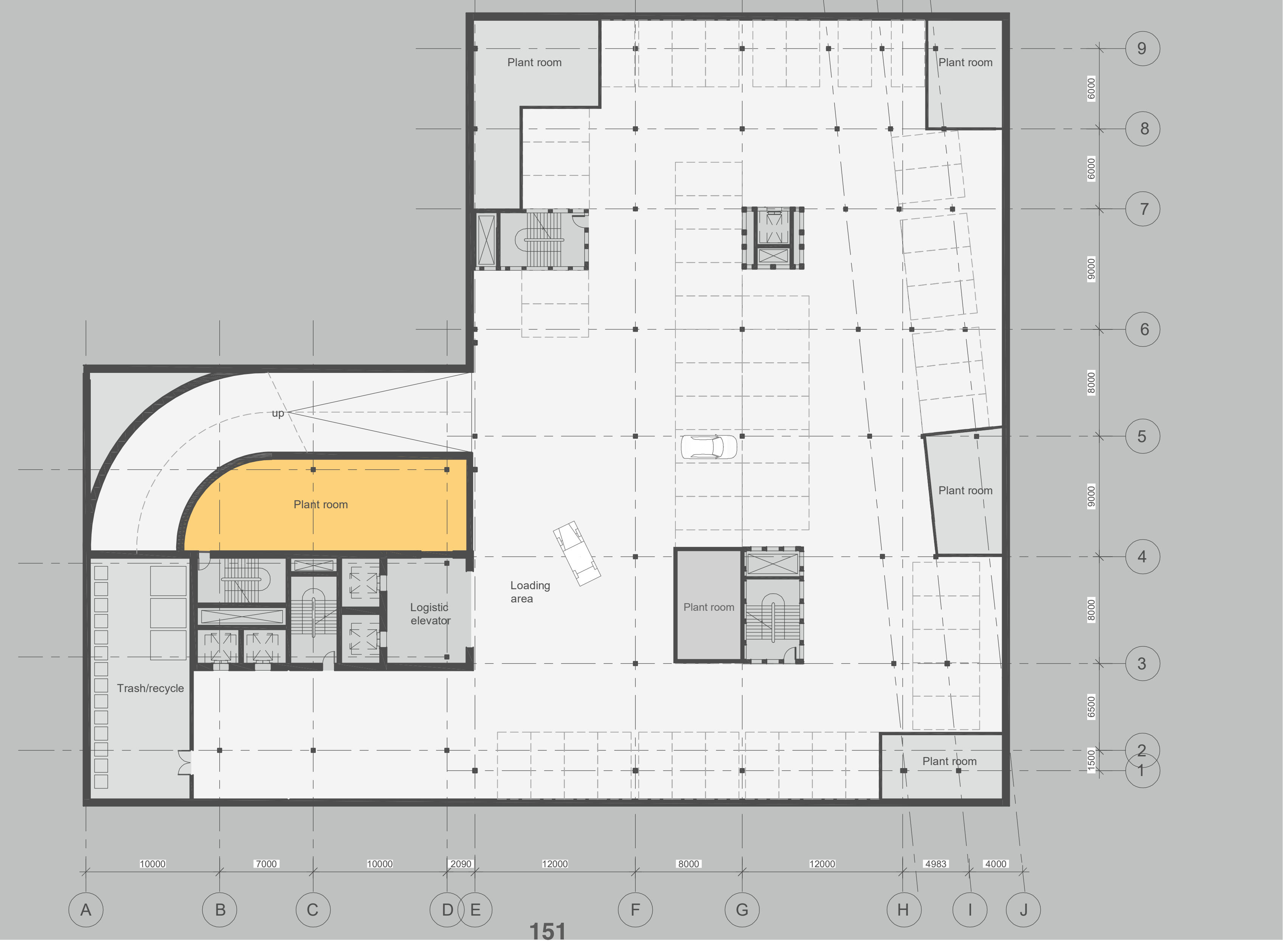




\section{Climate Scheme}

Podium

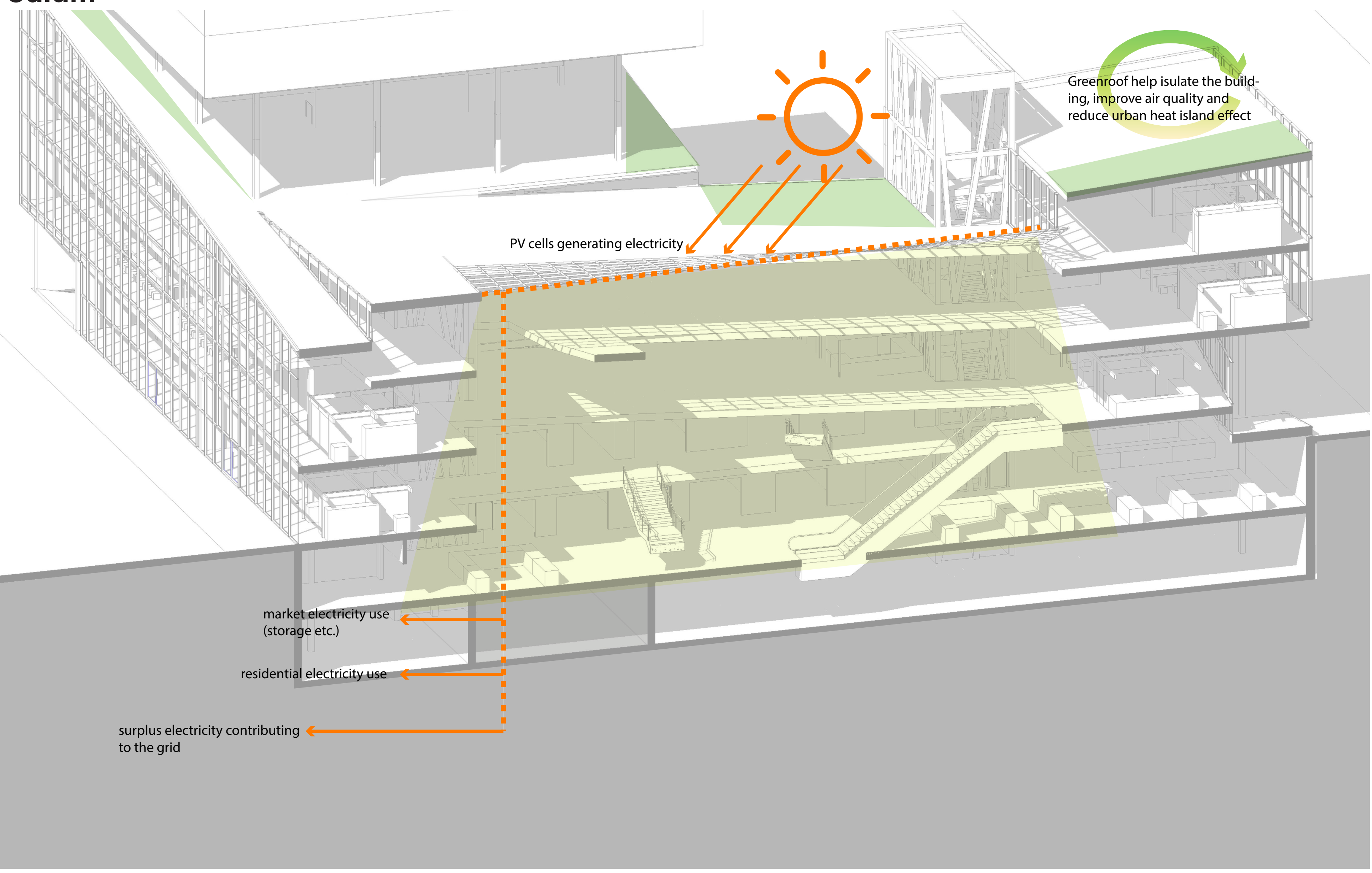




\section{Climate Scheme}

Podium

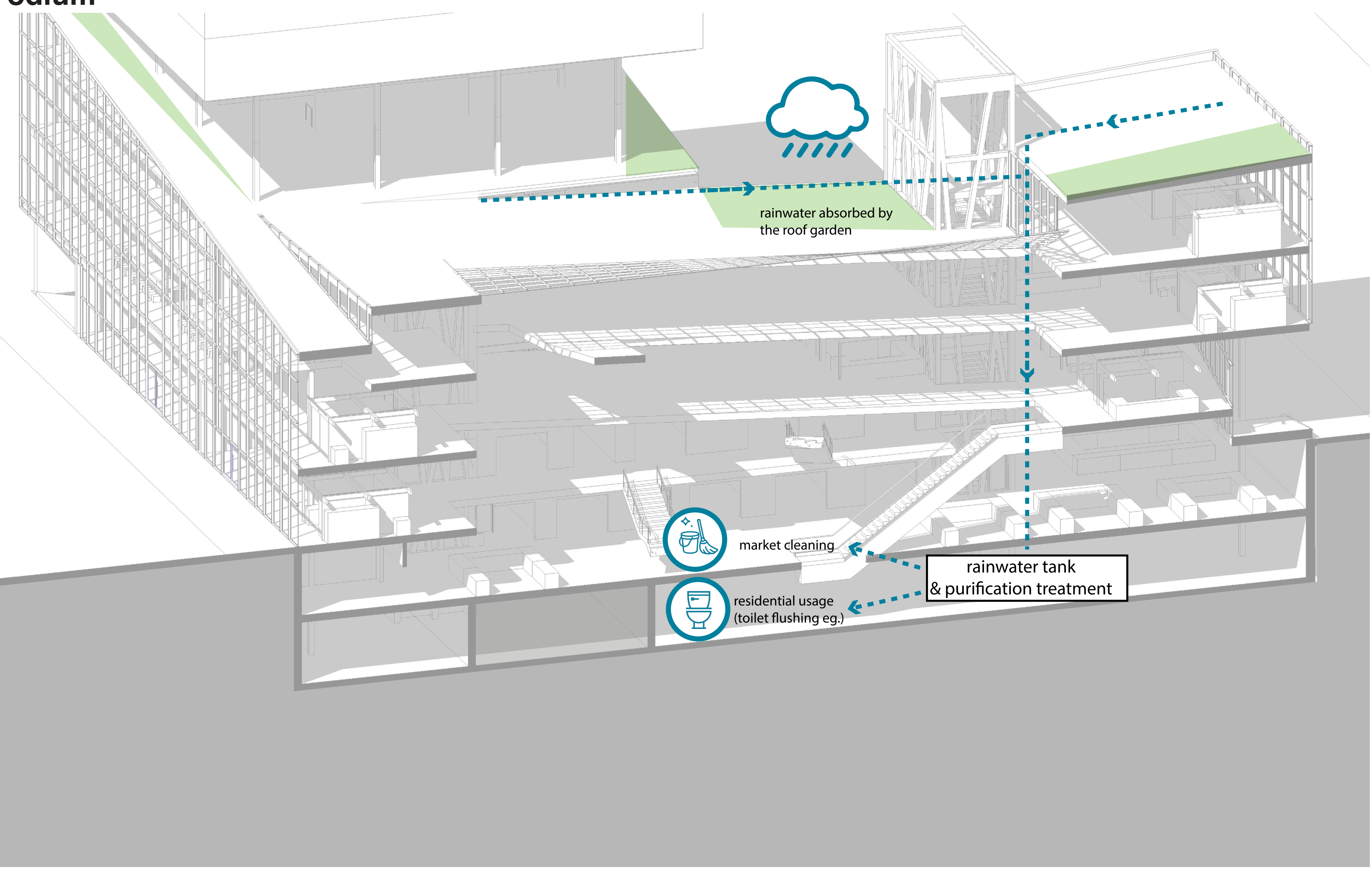




\section{Climate Scheme}

Podium

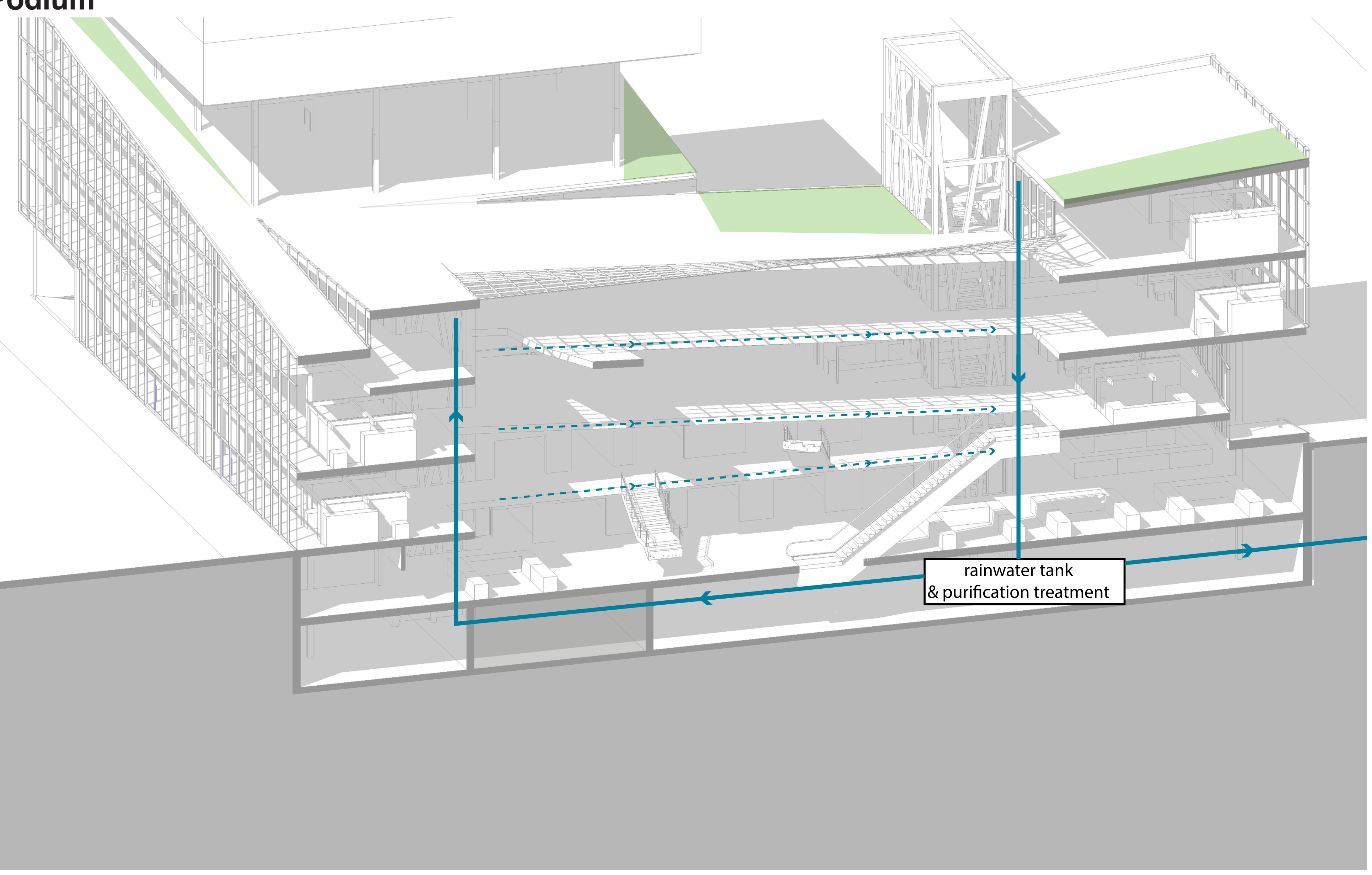


Climate Scheme

Tower

natural ventilation +mechanical ventilation
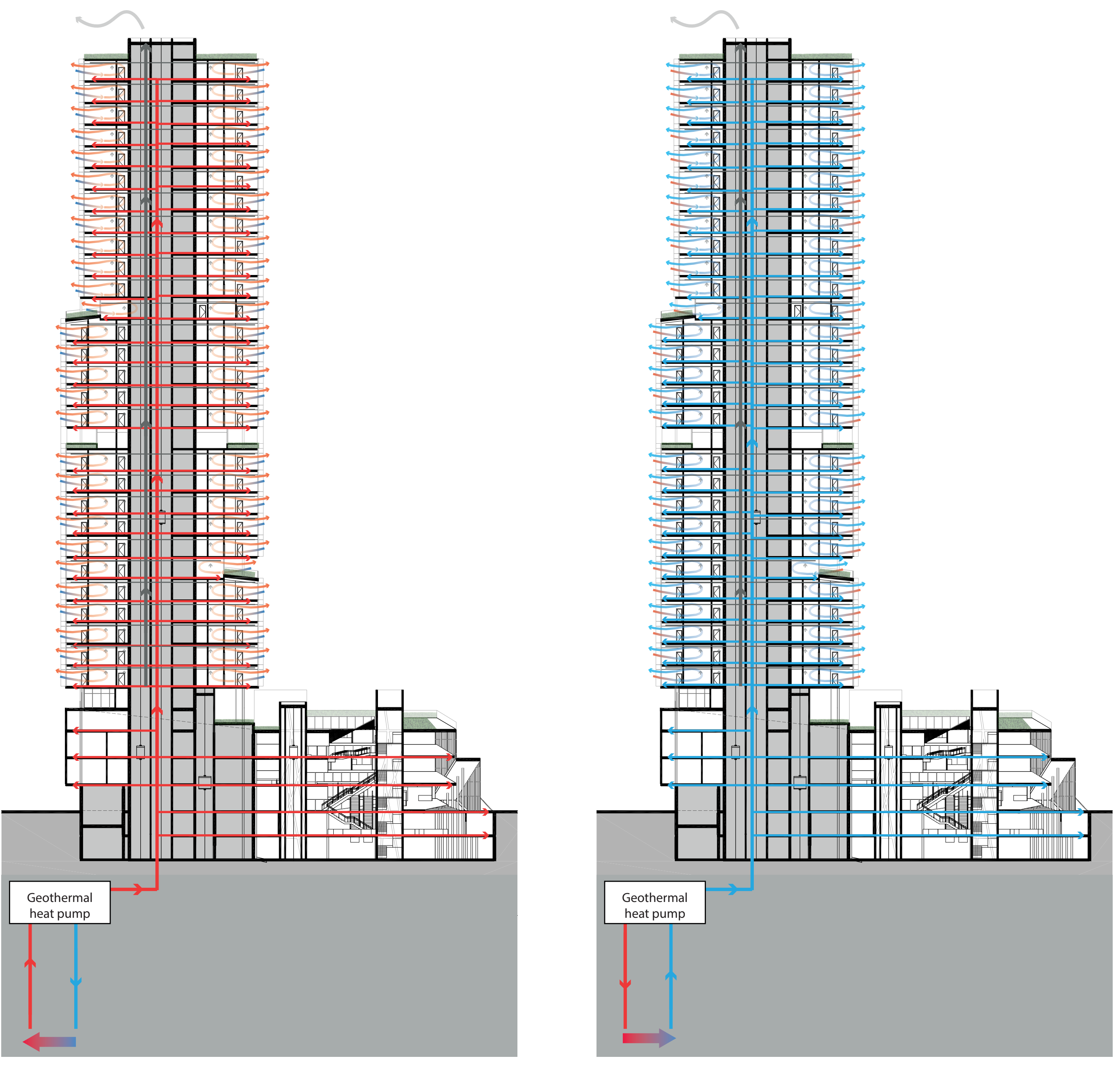
Climate Scheme

Tower

natural ventilation +mechanical ventilation summer sun

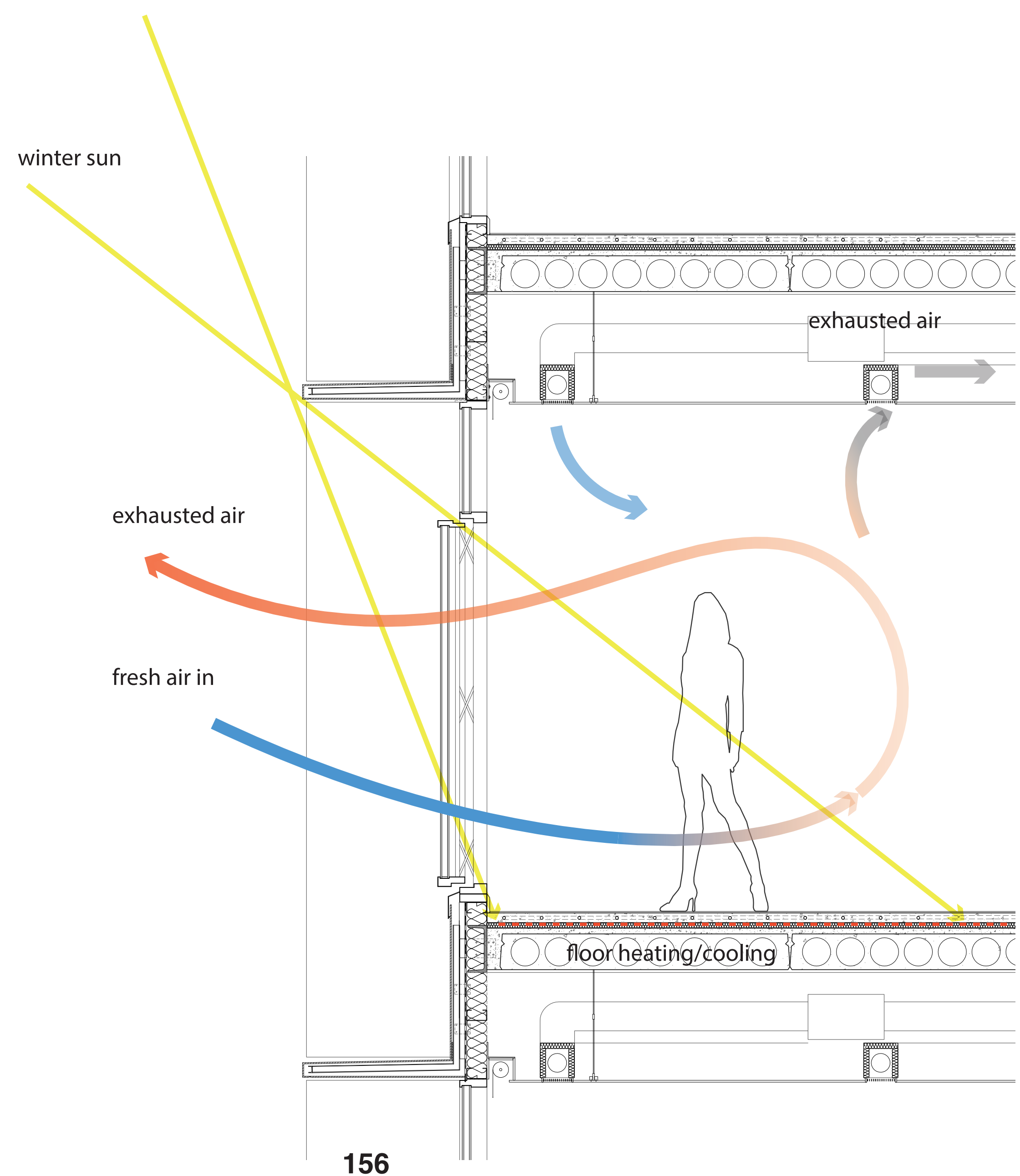




\section{Conclusion}




\section{Ambitions}

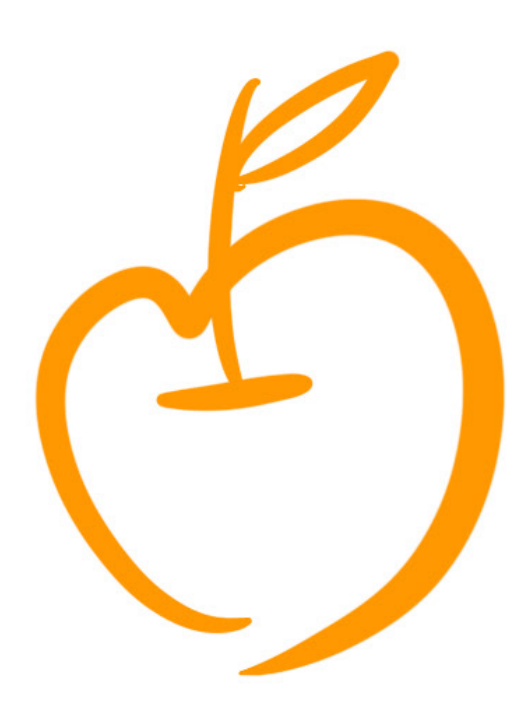

A food market provide the local community with fresh groceries

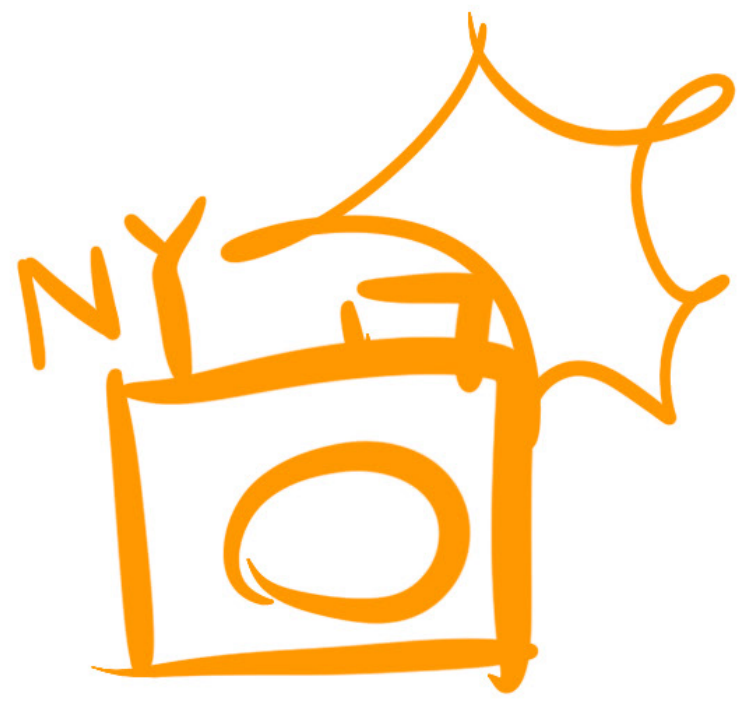

An iconic market provide tourists with unique shopping experience that is authentically

'New York'
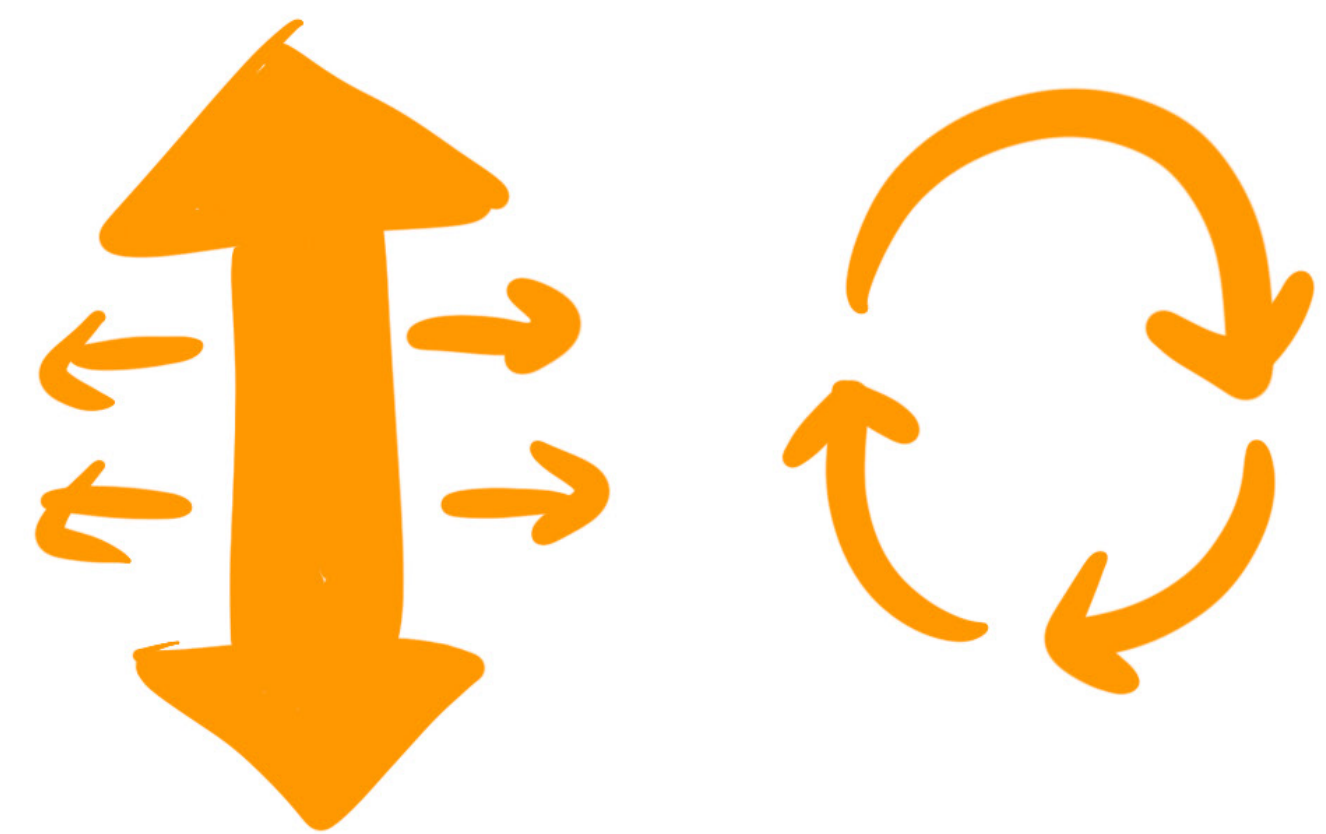

A market that is easily accessible A market promote a more sustainable lifestyle

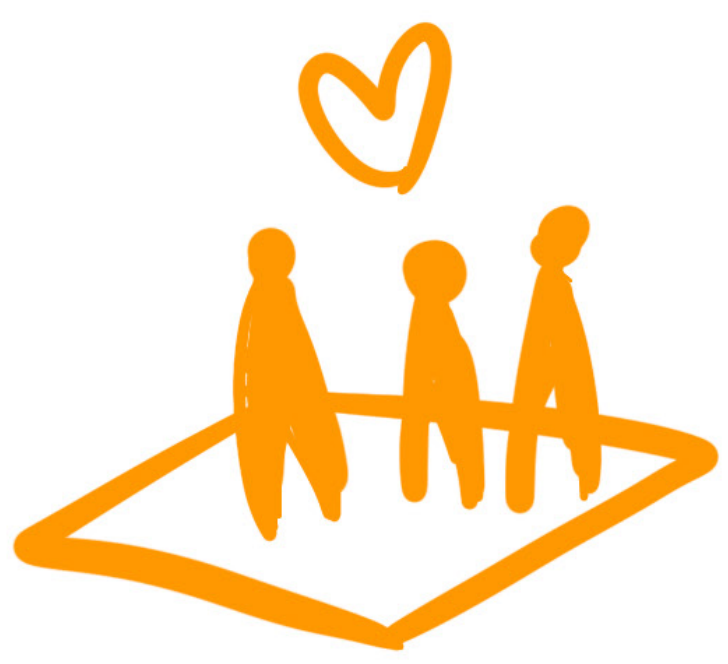

A market that is also a public space that is accessible for everyone 


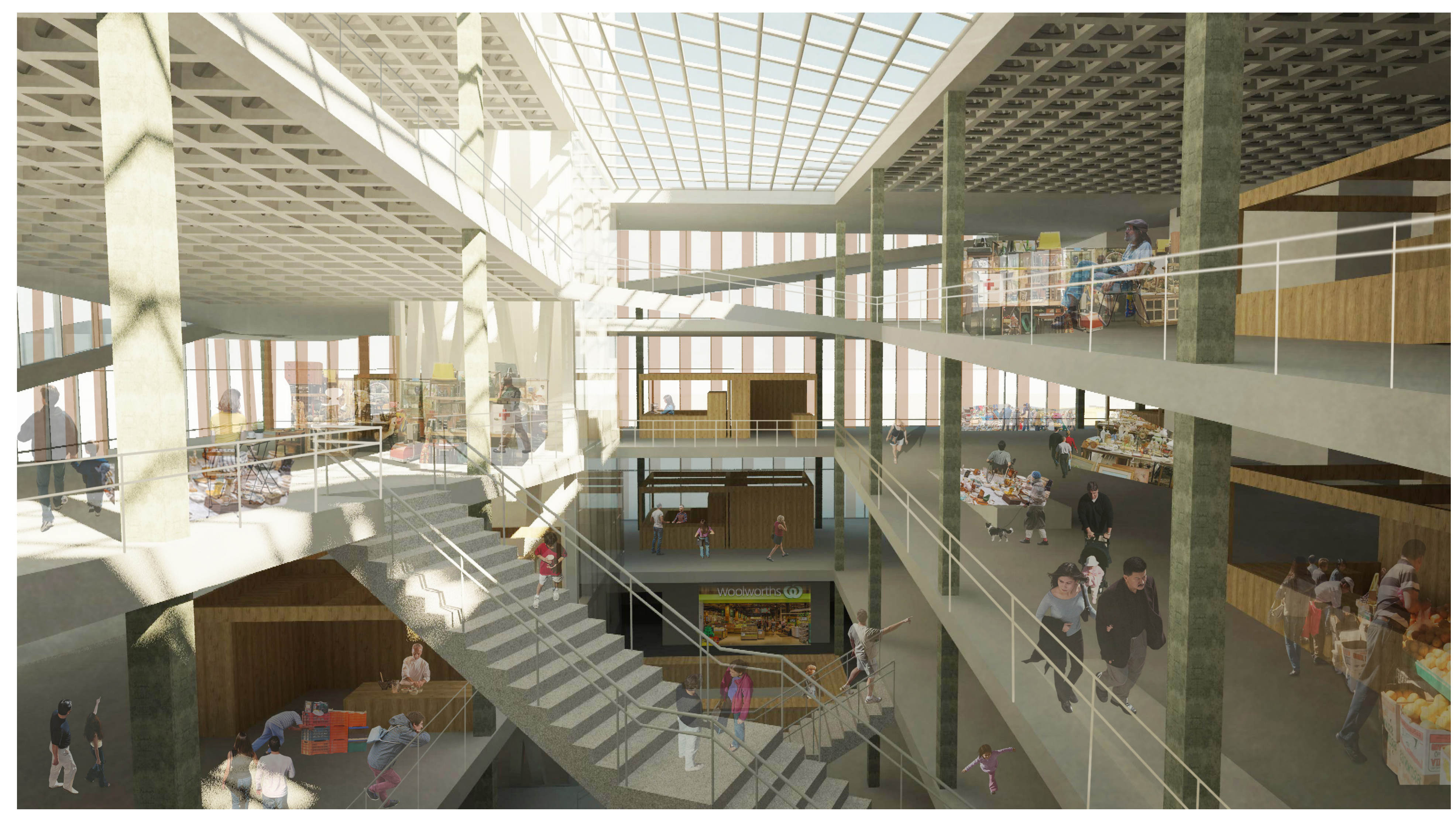




\section{Thank you!}

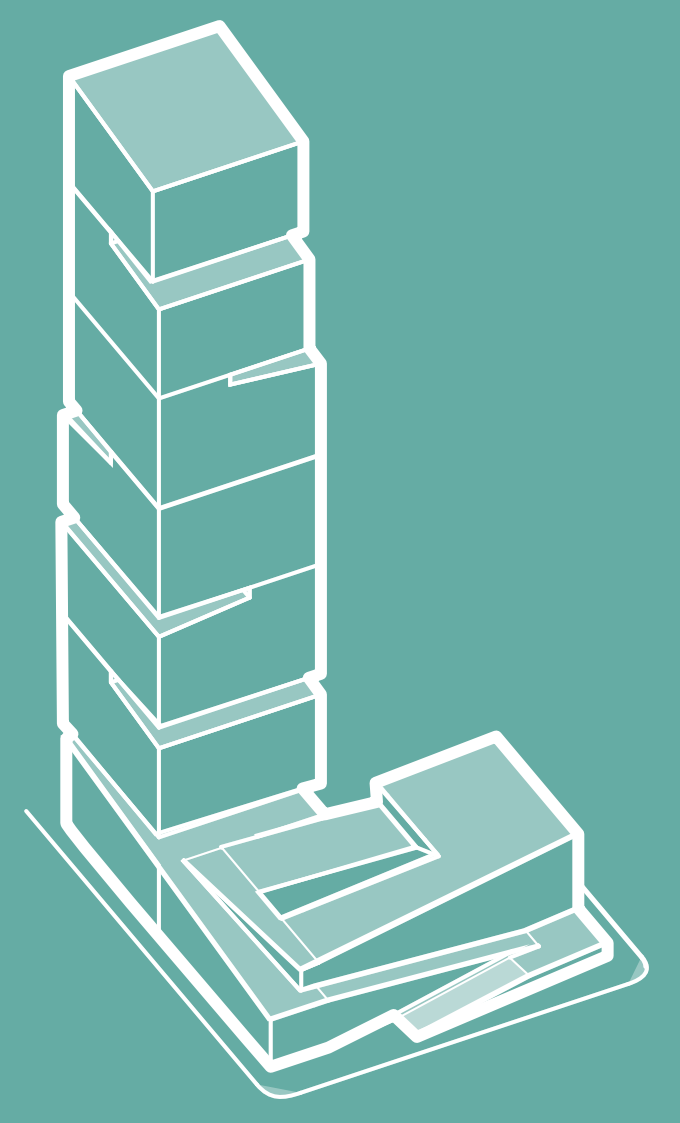

